Portland State University

PDXScholar

\title{
Assessing Well-being of Asian Elephants (Elephas maximus) Through Major Events and Transitions, with Development and Aging, and Husbandry Changes
}

Sharon Stuart Glaeser

Portland State University

Follow this and additional works at: https://pdxscholar.library.pdx.edu/open_access_etds

Part of the Biology Commons, and the Endocrinology Commons Let us know how access to this document benefits you.

\section{Recommended Citation}

Glaeser, Sharon Stuart, "Assessing Well-being of Asian Elephants (Elephas maximus) Through Major Events and Transitions, with Development and Aging, and Husbandry Changes" (2020). Dissertations and Theses. Paper 5545.

https://doi.org/10.15760/etd.7419

This Dissertation is brought to you for free and open access. It has been accepted for inclusion in Dissertations and Theses by an authorized administrator of PDXScholar. Please contact us if we can make this document more accessible: pdxscholar@pdx.edu. 
Assessing Well-being of Asian Elephants (Elephas maximus) Through Major Events and Transitions, with Development and Aging, and Husbandry Changes

by

Sharon Stuart Glaeser

A dissertation submitted in partial fulfillment of the requirements for the degree of

Doctor of Philosophy

in

Biology

Dissertation Committee:

Deborah Lutterschmidt, Chair

Nadja Wielebnowski, Co-Chair

David Shepherdson

Deborah Duffield

Janine Brown

Jason Newsom

Portland State University

2020 
(C) 2020 Sharon Stuart Glaeser 


\begin{abstract}
In this body of research, reproductive and adrenal hormones and behavior were used to evaluate individual and group responses to physiological, social, and environmental changes in zoo-housed Asian elephants, considering factors of sex, age, and life stage. Animals experience physiological, social, and environmental changes as part of their natural history and individual life experience. Measures of both positive and negative states are needed to assess the impact of these changes at the individual and group level. Such measures can help us better understand how animals cope with a changing environment, and can help inform management decisions. Through longitudinal analyses of more than 20 years of gonadal and adrenal hormone data (Chapters 2 and 3), we confirmed the presence of intrinsic glucocorticoid (GC) patterns associated with reproductive state in male and female Asian elephants, providing further evidence that GCs play a role in normal reproductive function. In females, circulating cortisol was higher in the follicular phase compared to the luteal phase of the ovarian cycle, and highest in lactational anestrous compared to all other reproductive states. In bulls, circulating cortisol covaried positively with testosterone and musth, as observed in previous studies of elephants and during the analogous condition of rut in other ungulates. Age-related changes in cortisol were observed in both sexes, and some individuals experienced higher rates of change in cortisol in one phase of their reproductive cycle versus another. In bulls, testosterone increased after puberty but decreased with advancing age. Concentrations of GCs covaried more consistently with physiological changes than with social changes in both sexes. Overall, the elephants in
\end{abstract}


these studies showed adaptive adrenal responses to change, and they also exhibited substantial individuality in adrenal response to social life events. These findings reinforce the need to assess welfare on an individual basis and to consider factors influencing the impact of perceived stressors, such as social support, temperament, and life history. The study conducted to monitor an elephant herd through transition to a new habitat (Chapter 4) provided further insights on habitat features that help us meet the physiological, psychological and social needs of elephants under human care, and helped identify which of those features may be most beneficial for enhancing welfare. The complexity and flexibility of the new habitat was effective in improving overall health and welfare by encouraging activity and providing meaningful challenges and the opportunity to express more appetitive behaviors. The new habitat also offered increased control over environmental conditions and social interactions, and the space and resource distribution helped to support changing herd dynamics and greater social equity. Overall, these studies helped deepen our understanding of Asian elephant physiology, and highlight the importance of taking intrinsic patterns of hormone secretion into account when evaluating the impact of external changes. New insights into the welfare impact of habitat and husbandry factors help inform future housing and management decisions. Finally, a better understanding of the impact of social change and resiliency in response to real and perceived stressors allows us to improve social management to enhance welfare in both captive settings and free-ranging environments. 


\section{DEDICATION}

To my Mom and Dad

Their love of nature and wildlife led me to begin this journey ... and they walked beside me as far as they could. 


\section{ACKNOWLEDGEMENTS}

My first round of thanks is for the elephant managers, keepers, researchers, veterinarians, and enthusiasts of the Elephant Managers Association, who welcomed me into the community of elephant professionals 15 years ago when I was transitioning careers with a 'dream to work with animals' and a fascination with elephant

communication. These people, and the elephants, have taught me so much and are forever in my heart.

Before I ever stepped foot in an elephant barn, Dr. Bets Rasmussen and Nancy Scott instilled in me an appreciation and respect for the keeper staff, as keepers make zoo research possible and their knowledge is invaluable. The hormone dataset summarized in this body of work is attributed to over 40 elephant handlers at both the Oregon Zoo and the National Zoological Park, who collected biological samples from individuals of each herd for over 25 years. We owe the integrity of these data to over 15 diligent technicians at the Oregon Zoo and the Smithsonian's Conservation Biological Institute (SCBI) endocrinology labs.

The behavior research would not have been possible without the dedication and tenacity of 14 Oregon Zoo volunteers who gave over 2000 hours of their time to this project, endured the cold, heat, rain, wind, mud, and construction, and continually showed enthusiasm for the opportunity to observe the animals so dear to their hearts. Having been a zoo volunteer myself, I cherish our chance meetings on zoo grounds and appreciate their continued interest in my work. 
I would like to extend my sincere gratitude to the Oregon Zoo Foundation for funding my Research Associate position, and for their enthusiasm in sharing my work with zoo supporters. Project funding was generously provided by the Oregon Zoo and the SCBI Endocrinology Lab, with additional grant support from the Portland State University (PSU) Forbes-Lea Endowment Fund. I also appreciate the support received from the PSU Marie Brown Graduate Student Travel Award.

To the Oregon Zoo elephant care staff - Bob Lee, Tarah Bedrossian, Dimas Dominguez, Shawn Finnell, Gilbert Gomez, Jason Miles, Joe Sabastiani, and Pam Starkey - I will forever appreciate your dedication and compassion in caring for the elephants I love, for supporting my work and not tiring of my many questions, and for your kindness and caring towards me and my family. You made me feel like a part of the team, and I cherish our times of both joy and sorrow. I am grateful to the Oregon Zoo veterinarian staff for their excellent care, and respectfully acknowledge their time and effort in sharing medical information pertinent to my work, and their patience with my pursuit to understand.

For my friends, advocates, and allies in science, your collaboration and friendship mean the world to me. You helped me through my most challenging days. Karen Lewis was a voice of reason, and helped me to apply my life experience to my collective scientific and conservation pursuits. She was a selfless collaborator and always willing to help. Thank you to Meghan Martin for taking the lead in a round of statistics when I was grieving and had a conference to prepare for, and for her ongoing generosity in sharing her knowledge. Many thanks to the remarkable team of Nicole Boisseau, Katie Edwards, Steve Paris, and Natalia Prado at SCBI for their guidance, inspiration, steadfast 
commitment to welfare and conservation. I appreciate their willingness to juggle a hundred balls... and still find time for laughter and celebration.

At Portland State University, the Biology Department serves as a foundation of inspiration and support, with amazing professors and an array of unique learning opportunities. I join many before me in thanking Roxanne Treece and the PSU Office of Graduate Studies for their governance and proactive communication.

My sincere thanks to Oregon Zoo Director Dr. Don Moore and Deputy Director Sheri Horiszny for their dedication to animal welfare and conservation, for the opportunity to represent the Oregon Zoo in elephant care and conservation programs in the Malaysian state of Sabah on Borneo, and for their support of unique projects to this aim. Thank you to Oregon Zoo Curator Bob Lee for partnering with me in these efforts, for believing in my abilities, and promoting my achievements. Many thanks to the Oregon Zoo Marketing/Communications team for their enthusiasm in sharing my research with the broader community, for polishing my presentations, and coaching me with interviews. Lastly, a heartfelt thanks to the Oregon Zoo community at large for their devotion to the animals entrusted to their care, for their interest in my work, and for years of support and friendship.

My $\mathrm{PhD}$ committee deserves my deepest gratitude and respect. I admire their remarkable breadth and depth of knowledge and experience, and I am fortunate to learn from such accomplished professionals. I also sincerely appreciate their balance of prodding and praise, which got me through each milestone and to this finish line in the long five years that now seems to have flown by. Dr. Deborah Lutterschmidt is a devoted advisor and advocate, and was always available to help navigate challenges. Dr. Nadja 
Wielebnowski and Dr. David Shepherdson encouraged me to apply for this PhD program, and provided opportunities and promoted my accomplishments throughout my tenure at the Oregon Zoo. I also thank them for helping to advance the welfare of zoo animals worldwide. Dr. Deborah Duffield provided invaluable guidance throughout this academic pursuit. Dr. Janine Brown is my mentor in elephant research, and her support and guidance over many years has facilitated my achievements and opened many doors of opportunity. Many thanks to my Graduate Office Representatives, Dr. Angela Strecker and Dr. Jason Newsom, for their guidance and oversight, and their unique perspectives from different disciplines of science.

Finally, thank you to my husband, Bob, who is my pillar of strength. Thank you to my family and friends who walked beside me, and to the countless kindred spirits who inspired me on this magnificent journey. 


\section{TABLE OF CONTENTS}

ABSTRACT

DEDICATION

iii

ACKNOWLEDGEMENTS iv

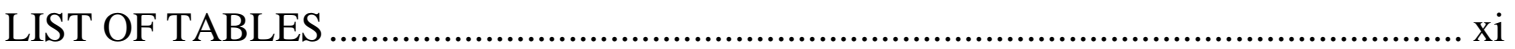

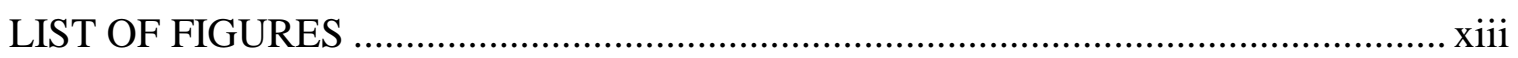

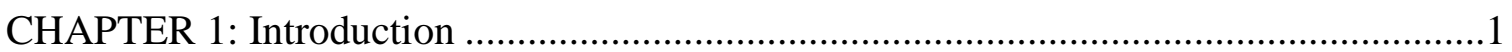

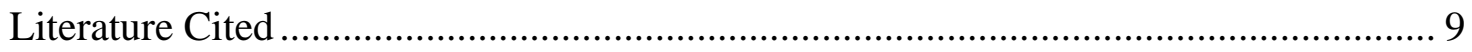

CHAPTER 2: Effects of physiological changes and social life events on adrenal glucocorticoid activity in female Asian elephants (Elephas maximus)..... .15

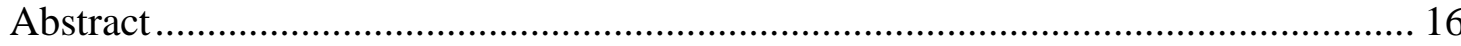

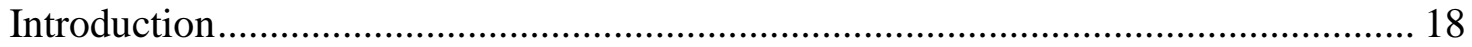

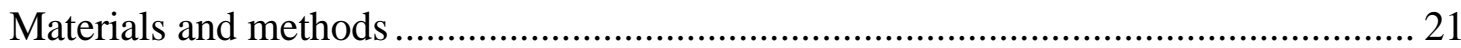

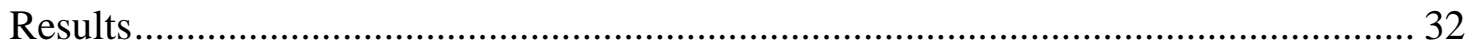

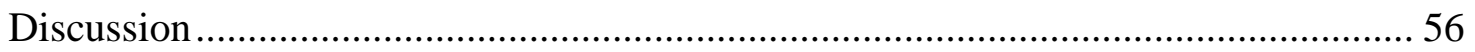

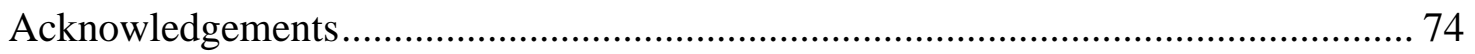

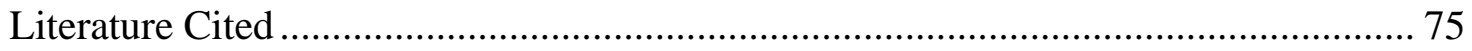

CHAPTER 3: Adrenal glucocorticoid activity associated with musth, aging, social changes, and medical treatment in adult male Asian elephants (Elephas maximus) ..........88

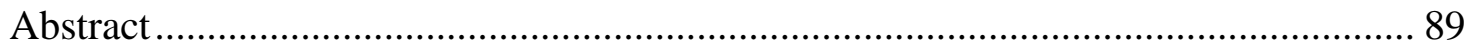

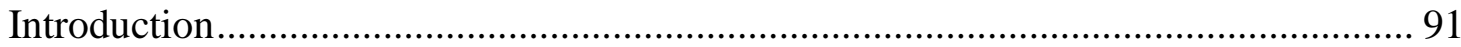

viii 
Materials and methods

Results

Discussion

Acknowledgements.

Literature Cited

CHAPTER 4: Supporting Asian elephant (Elephas maximus) welfare and herd dynamics with a more complex and expanded habitat at the Oregon Zoo

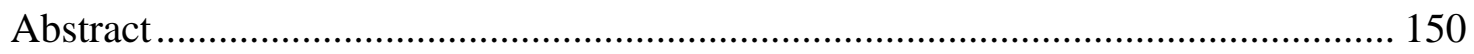

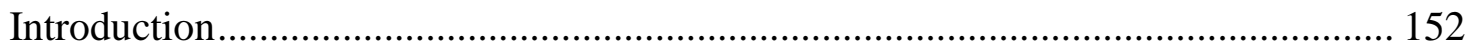

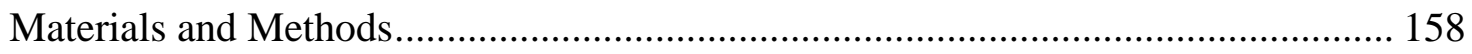

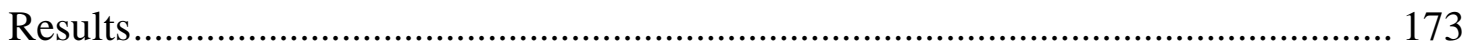

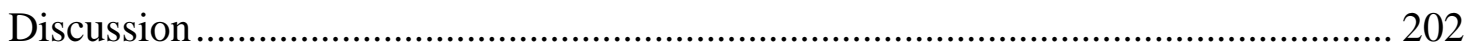

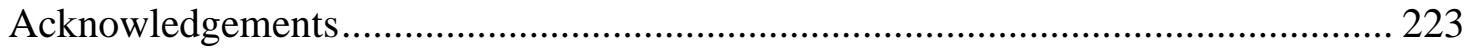

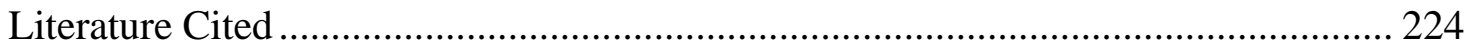

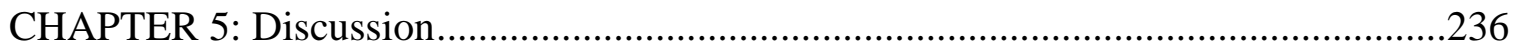

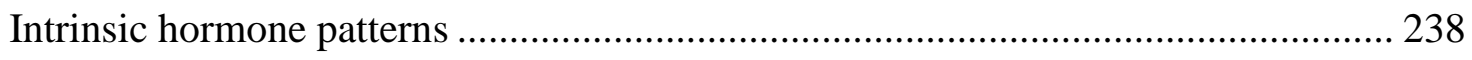

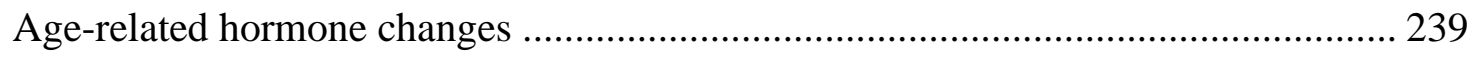

Adrenal response to social and environmental change ..................................... 241

Supporting welfare and herd dynamics through habitat design ............................ 244

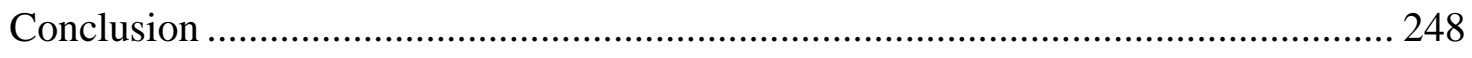

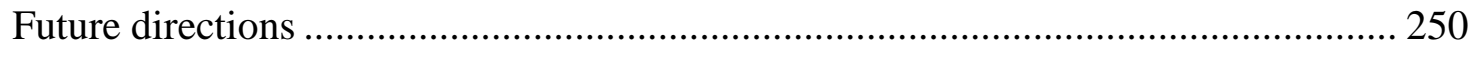


Appendix A: Longitudinal Hormone Concentrations (Chapter 2) ...............................262

Appendix B: Charted Predictions for Pairwise Comparisons (Chapter 2) .....................268

Appendix C: Elephant Behavior Ethogram (full version) (Chapter 4) .........................272

Appendix D. Mann-Whitney U-test for pairwise comparisons (Chapter 4)...................282 


\section{LIST OF TABLES}

Chapter 2 Tables

Table 1: Female elephants included in this study. 22

Table 2: Ovarian cycle phase as a predictor of adrenal GC activity. 38

Table 3: Age as a predictor of adrenal GC activity. 42

Table 4: Birth to a herdmate as a predictor of adrenal GC activity................................ 46

Table 5: Death to a herdmate as a predictor of adrenal GC activity............................. 47

Table 6: Transfer of a herdmate as a predictor of adrenal GC activity......................... 52

Table 7: Transfer of self as a predictor of adrenal GC activity. ................................. 54

Table 8: Death of self as a predictor of adrenal GC activity. ..................................... 56

\section{Chapter 3 Tables}

Table 1: Male elephants included in this study. 95

Table 2: Musth behavior scale for Oregon Zoo elephants ....................................... 100

Table 3: Temporal Gland Swelling and Secretion (TGS) ...................................... 102

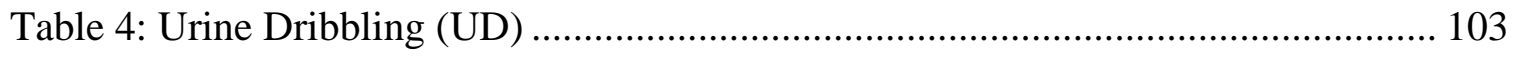

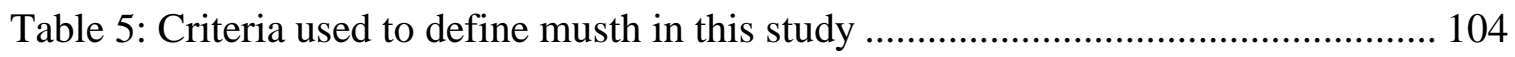


Table 6: Serum testosterone concentration $(\mathrm{ng} / \mathrm{ml})$ for males across all reproductive states and during periods of musth and inter-musth.

Table 7: Serum testosterone concentrations $(\mathrm{ng} / \mathrm{ml})$ corresponding to the beginning and end of musth.

Table 8: Age as a predictor of testosterone.

Table 9: Serum cortisol concentration $(\mathrm{ng} / \mathrm{ml})$ for males across all reproductive states and during periods of musth and inter-musth. 118

Table 10: Testosterone and musth as a predictor of adrenal GC activity. 119

Table 11: Age as a predictor of adrenal GC activity. 121

Table 12: Social life events as predictors of adrenal GC activity. 124

Table 13: TB detection and anti-TB therapy as predictors of adrenal GC activity. ....... 126

Table 14: Health decline leading to natural death or euthanasia as a predictor of adrenal GC activity.

Chapter 4 Tables

Table 1: Elephants included in this study 158

Table 2: Elephant Behavior Ethogram-abbreviated version

Table 3: FGM concentrations (ng/g) in each habitat phase.

Table 4: Statistical results comparing median FGM concentrations across and between habitat phases. 182

Table 5: Statistical results comparing variability in FGM concentrations across and between habitat phases. 183 


\section{LIST OF FIGURES}

Chapter 2 Figures

Fig 1: Reproductive state as a predictor of adrenal GC activity

Fig 2: Ovarian cycle phase as a predictor of adrenal GC activity.

Fig 3: Age as a predictor of adrenal GC activity. ................................................... 44

Fig 4: Death of a herdmate as a predictor of adrenal GC activity. .............................. 50

Fig 5: Facility transfer as a predictor of adrenal GC activity. .................................... 55

\section{Chapter 3 Figures}

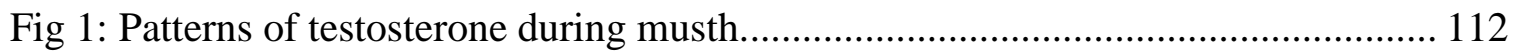

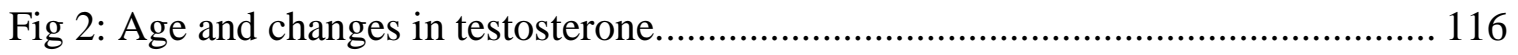

Fig 3: Age and changes in testosterone comparing GLMM predications and calculations.

Fig 4: Age as a predictor of adrenal GC activity.

122

\section{Chapter 4 Figures}

Fig 1: Study timeline. 159

Fig 2: New habitat with outdoor area shaded using ArcMap 163 
Fig 3: Mean daily walking distance comparing the previous habitat and new habitat... 174

Fig 4: Representative examples of GPS data in outdoor areas of the new habitat at

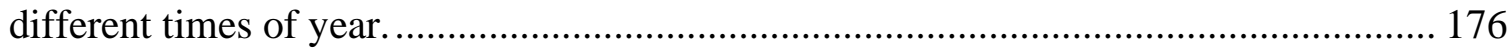

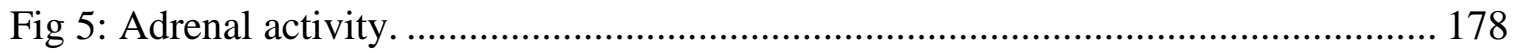

Fig 6: Hormone profile of FGM concentrations for F-calf showing temporal relationship

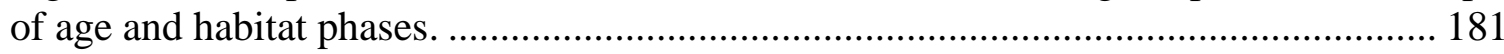

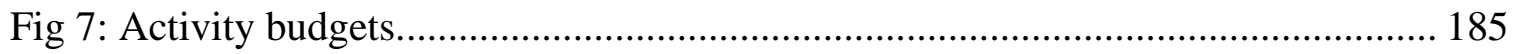

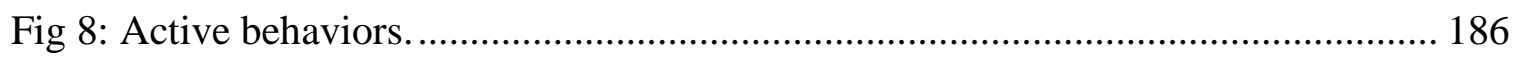

Fig 9: Hill Number analysis results showing intersecting diversity profiles ................. 189

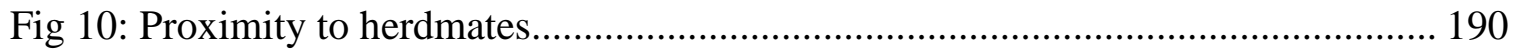

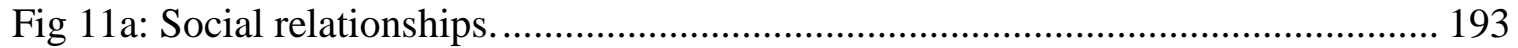

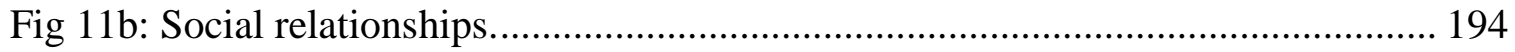

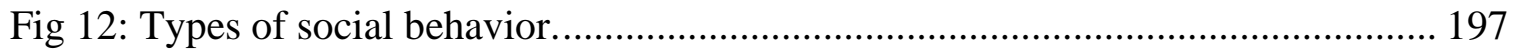

Fig 13: Interactions with resources in the previous habitat. .................................... 200

Fig 14: Interactions with resources in the new habitat. ......................................... 201 


\section{CHAPTER 1: Introduction}

Asian elephants (Elephas maximus) are classified as Endangered under the International Union for Conservation of Nature and Natural Resources (IUCN) Red List of Threatened Species (Choudhury et al., 2008). The primary threats across their range are habitat loss, degradation, and fragmentation, which are driven by an expanding human population, and lead in turn to increasing conflicts between humans and elephants (IUCN, 2020). The global wild population of about $41,000-52,000$ is currently under assessment by the IUCN Species Survival Commission, Asian Elephant Specialist Group. The population of Asian elephants under professional care in western facilities includes 228 (53 males, 175 females) in 46 facilities in North America and Mexico, and 298 (84 males, 214 females) in 72 facilities in Europe, as recorded in the Association of Zoos and Aquariums Regional Studbook for Asian elephants (Lee, 2019), and the European studbook for Asian elephants (van Wees and Damen, 2015), respectively.

Animals in the wild and in human care experience many changes as part of their natural history. Physiological changes occur with development and aging, reproductive state, injury and illness. In addition, long-lived social animals experience events and transitions in their social and physical environments throughout their lifetime, such as births and deaths of conspecifics, changes in social structure and dominance rank, and seasonal movements. With increasing human influence on free-ranging wildlife, animals are experiencing changes with potential for both negative and positive impact - disruption to daily and seasonal routes, measures to reduce conflict including relocation and 
translocation, human-caused injury, treatment for injury and illness in-situ or capture from the wild for treatment ex-situ, rehabilitation for release or transition to captivity. For animals under human care in western zoos and in range countries, events and transitions include facility transfers, modifications to existing facilities, changes in social structure and in caretakers, major changes in husbandry practices, and change in roles such as an ambassador animal engaging with visitors or being on exhibit. Objective measures of both positive and negative states are needed to assess the impact of these changes at the individual and group level to help us better understand how animals cope with a changing environment, and to provide important information for animal management decisions.

Welfare of wildlife, whether free-ranging or managed to varying degrees under human care, has become an increasingly important consideration for conservation and breeding efforts worldwide. From a conservation perspective, individuals with good health and welfare generally experience increased survivorship and reproduction, and thus are better able to contribute to population sustainability. Modern zoos strive to provide environments in which individuals thrive and lead high-quality lives, while serving as conservation ambassadors for their wild counterparts, to impassion zoo visitors to help conserve the natural world. Employing evidence-based conservation and captive management practices is therefore critical, and even more so for elephants in light of current issues and priorities. Declining in-situ populations and increasing human impacts (IUCN, 2020) are leading to more intensively managed in-situ populations (Cameron and Ryan, 2016), and there is evidence that wild elephants are changing their behavior with 
increasing human disturbance (Vidya and Sukumar, 2005; Othman et al., 2019).

Quantifying these changes needs further attention. Modern zoos and aquariums are required to provide a high standard of animal care and aim for continuous improvements in animal care and welfare (Watters and Wielebnowski, 2009; Meehan et al., 2016b; Brando and Buchanan-Smith, 2018). Elephants present unique challenges in the captive environment (Veasey, 2006), and there is increasing public focus on philosophical issues of animal rights and criticism regarding animal welfare (Whitham and Wielebnowski, 2013; Williams et al., 2018), with debates surrounding adaptability of elephants to the captive environment and inconclusive benchmarking (Veasey, 2006; Mason and Veasey, 2010).

A growing number of studies are taking an epidemiological approach to examine factors in the captive environment associated with both positive and negative welfare outcomes in elephants in western zoos (Harris et al., 2008; Proctor and Brown, 2015; Meehan et al., 2016a; Brown et al., 2019; Carlstead et al., 2019; Edwards et al., 2019) and in tourist elephants in Asia (Norkaew et al., 2018; Bansiddhi et al., 2019; Norkaew et al., 2019). Commonalities in findings across vastly different management systems (Brown et al., 2020) reinforce that essential needs must be met regardless of management strategy, and there is no "standard" management that will guarantee good welfare for all elephants across all life stages. Although epidemiological analyses are by nature correlational, the results provide a basis for further investigations in how individuals and groups respond to these factors. 
Animal welfare science in zoos aims to determine the best husbandry practices to promote welfare of individuals across life stages. Understanding how factors in the captive environment affect individual animal health and welfare can be aided by longterm monitoring of biological functioning. Long-term monitoring helps establish baseline values for individuals, populations, and species, and allows for detection of patterns that are important to consider when evaluating responses to external stimuli. In this dissertation research, long-term assessments of reproductive and adrenal hormones and behavior in zoo-housed Asian elephants were conducted to elucidate patterns associated with physiological changes, and assess responses to major social and environmental changes at an individual and group level. Common concepts applied throughout this dissertation include 'animal welfare', the 'stress response', and 'intrinsic biological patterns'. In the following I briefly outline how these concepts are defined as part of this specific dissertation research.

Although there are numerous definitions of animal welfare, for the purpose of the zoo-based projects conducted as part of this research, we use the definition that has been approved by Association of Zoos and Aquariums (AZA Accreditation Guidelines, 2019). Animal welfare refers to an animal's collective physical, mental, and emotional states over a period of time and is measured on a continuum from poor to excellent over an animal's lifetime. Central to this welfare framework is that welfare indicators are quantitative measurements that include both positive and negative affective states, and are primarily animal based rather than resource based (Whitham and Wielebnowski, 2013). Types of welfare indicators include behavior (e.g., species-appropriate behaviors, activity level, 
rest and sleep, time budgets), physiology (e.g., reproductive hormones, reproduction, adrenal hormones, metabolic and immune biomarkers), and physical appearance and health (e.g., body condition, muscle tone, species-specific health parameters such as skin, fur, or feather condition). Some important factors influencing an individual's welfare state are the natural history of the species, the history and experiences of the individual, genetics and temperament, and a wide variety of animal management and environmental factors.

The stress or adrenal response, often used as a potential indicator of animal health and welfare, is an adaptive response (to a real or perceived stressor) whereby a suite of behavioral, physiological and neuroendocrine changes occur to help an organism cope with a real or perceived stressor and re-establish homeostasis (Moberg, 2000; Möstl and Palme, 2002; Nelson, 2011). The most commonly used bio-markers of stress are glucocorticoids (GC), which are secreted from the adrenal cortex through activation of the hypothalamic-pituitary adrenal (HPA) axis in response to a variety of stimuli (Möstl and Palme, 2002; Ralph and Tilbrook, 2016; Palme, 2019). The primary roles of GCs at basal levels are energy regulation and mobilization, but higher concentrations of GCs facilitate physiological changes associated with the stress response (Busch and Hayward, 2009). Adaptive and normal adrenal responses (i.e., eustress) can provide one potential indicator of positive welfare, although welfare studies have historically focused on assessment of stress (i.e., distress) as an indicator of negative welfare. Short-term stressors (e.g., mating, fighting, hunting, predator avoidance) cause an acute response; however, prolonged exposure to psychological or physical stressors (e.g., social 
aggression, lack of nutrition) can result in a chronic stress response with continually high concentrations of GCs. Such chronic stress may have deleterious effects on animal health and well-being including immunosuppression, decreased wound healing, increased susceptibility to disease, poor reproduction, and development of stereotypic behaviors (Moberg, 2000; Romero, 2004). GCs can be measured in serum, urine, feces, saliva, and hair. Serum provides a measure of circulating hormones, which represents an immediate state. This immediate state of serum is a composite of three components: endogenous cycles (circadian, seasonal, or normal fluctuation with reproductive cycle), immediate prior experience (e.g., actual or perceived acute stressors) and longer-term experience (chronic stressors) (Sheriff et al., 2011). GCs in the plasma are metabolized by the liver and excreted into the urine via the kidneys or into the gut via the bile ducts; however, there can also be native GCs in the urine (reviewed in (Sheriff et al., 2011)), and native urinary cortisol has been quantified in elephants (Brown et al., 2010). In elephants, the estimated lag time between hormone secretion and excretion (or transit excretion rate) is a few hours for urine (Brown et al., 2010), and approximately 24 hours for feces (Palme et al., 2000; Fuller et al., 2011). Although studies of stress biology rely heavily on GCs as biomarkers, measures of GC levels alone are not sufficient to determine whether a given stress response has positive or negative effects on an individual, and other indicators such as behavior or physical condition should be included in the assessment (Wielebnowski et al., 2002; Shepherdson et al., 2004; MacDougall-Shackleton et al., 2019). It is also important to consider whether stressful stimuli may be primarily regarded as positive (e.g., birth, excitement, mating) or negative (e.g., fear, anxiety, pain) in the larger context 
of an animal's life (Wielebnowski, 2003; Ralph and Tilbrook, 2016; McCormick and Romero, 2017).

Biological patterns that originate within an organism constitute 'intrinsic' or 'endogenous' patterns (Withers, 1992). Although these terms are often used interchangeably (Scheer et al., 2008; Zhdanova et al., 2011), there is an important difference with regards to environmental influence. Intrinsic and endogenous processes are both internally-driven. Intrinsic processes occur without environmental input; for example, changes in reproductive hormones associated with puberty, and covariance of reproductive and adrenal hormones. Endogenous processes originate intrinsically, but are entrained or triggered by environmental signals; for example, the circadian rhythm (approximately 24 hours) in sleep-wake cycles is an endogenous process entrained to the light. In this body of work, we measured variation in adrenal activity associated with physiological changes (e.g., reproductive state, phases of the reproductive cycle, aging), and determined whether the pattern in variation was consistent and predictable, and thus constituting an intrinsic hormone pattern.

In Chapters 2 and 3, we examined the effects of physiological changes and social life events on adrenal GC activity in zoo-housed female and male Asian elephants utilizing a longitudinal dataset comprised of over 20 years of serum hormone data and animal records, which is unmatched in duration and frequency of collection. The study group included elephants that were zoo-born and wild-born, breeding and non-breeding, and encompassing puberty through reproductive senescence, with an age range of 4 to 71 
years in females and 12 to 54 years in males. All elephants in these studies had a history of normal reproductive cycling, providing a unique opportunity to examine possible intrinsic patterns in reproductive and adrenal hormones, and account for these patterns in assessing individual differences in adrenal response to various stimuli.

In females (Chapter 2), we examined variation in adrenal GC activity with reproductive state and across the ovarian cycle, and assessed adrenal response to social life events such as births, deaths, and facility transfers (i.e., transfers in and out of the study facility) of herdmates. In males (Chapter 3), we characterized longitudinal patterns of testosterone and cortisol in relation to musth and age, and assessed adrenal responses to social changes, tuberculosis (TB) treatment, and health declines. Musth is a physiological and behavioral phenomenon in male elephants characterized by increased testosterone, heightened aggression or unpredictability, and increased sexual behavior; as such, musth can create both human safety and animal welfare concerns.

In Chapter 4, we evaluated the response to a major environmental change at both the individual and herd level. We conducted a four-year study at the Oregon Zoo to monitor elephant welfare during the transition from the old habitat, throughout the construction period, and finally the move to and the acclimation in the new habitat. We evaluated the effectiveness of the new habitat in achieving elephant program goals of increasing activity, promoting species-typical behaviors, offering increased opportunities for choice, and providing biologically meaningful challenges. Welfare indicators included 
reproductive and adrenal hormone analyses, detailed longitudinal behavior assessments, and distance walked through GPS monitoring.

Outcomes from these studies help elucidate underlying conditions or states that may affect adrenal GC activity, and the variation we can expect with physiological changes versus those in which the response is individualistic and event based. Results highlight the importance of accounting for intrinsic hormone patterns when evaluating the adrenal response to external stimuli, which is especially important when management decisions are based on these evaluations. Overall, these longitudinal analyses help to advance our knowledge on reproductive biology, health and welfare of Asian elephants, and on how social and environmental change may affect elephant welfare, both positively and negatively. Finally, a better understanding of the impact of major events and transitions, and resilience to perceived stressors, can be beneficial in determining the welfare needs of individuals and groups of elephants managed under human care and in free-ranging conditions.

\section{Literature Cited}

Bansiddhi, P., J. L. Brown, J. Khonmee, T. Norkaew, K. Nganvongpanit, V. Punyapornwithaya, T. Angkawanish, C. Somgird, and C. Thitaram. 2019. Management factors affecting adrenal glucocorticoid activity of tourist camp elephants in Thailand and implications for elephant welfare. PLoS One 14(10):e0221537. doi: 10.1371/journal.pone.0221537

Brando, S., and H. M. Buchanan-Smith. 2018. The 24/7 approach to promoting optimal welfare for captive wild animals. Behavioural Processes 156:83-95. doi: https://doi.org/10.1016/j.beproc.2017.09.010 
Brown, J., P. Bansiddhi, J. Khonmee, and a. Thitaram. 2020. Commonalities in Management and Husbandry Factors Important for Health and Welfare of Captive Elephants in North America and Thailand. Animals 10:737. doi: 10.3390/ani10040737

Brown, J. L., K. Carlstead, J. D. Bray, D. Dickey, C. Farin, and K. Ange-van Heugten. 2019. Individual and environmental risk factors associated with fecal glucocorticoid metabolite concentrations in zoo-housed Asian and African elephants. PLoS One 14(9):e0217326. doi: https://doi.org/10.1371/journal.pone.0217326

Brown, J. L., D. C. Kersey, E. W. Freeman, and T. Wagener. 2010. Assessment of diurnal urinary cortisol excretion in Asian and African elephants using different endocrine methods. Zoo Biol 29(2):274-283. doi:

https://doi.org/10.1002/zoo.20268

Busch, D. S., and L. S. Hayward. 2009. Stress in a conservation context: A discussion of glucocorticoid actions and how levels change with conservation-relevant variables. Biol Conserv 142(12):2844-2853. doi: https://doi.org/10.1016/j.biocon.2009.08.013

Cameron, E. Z., and S. J. Ryan. 2016. Welfare at Multiple Scales: Importance of Zoo Elephant Population Welfare in a World of Declining Wild Populations. PLoS One 11(7):e0158701. doi: 10.1371/journal.pone.0158701

Carlstead, K., S. Paris, and J. L. Brown. 2019. Good keeper-elephant relationships in North American zoos are mutually beneficial to welfare. Appl Anim Behav Sci 211:103-111. doi: https://doi.org/10.1016/j.applanim.2018.11.003

Choudhury, A., D. K. Lahiri Choudhury, A. Desai, J. W. Duckworth, P. S. Easa, A. J. T. Johnsingh, P. Fernando, S. Hedges, M. Gunawardena, F. Kurt, U. Karanth, A. Lister, V. Menon, H. Riddle, A. Rübel, and E. Wikramanayake. 2008. Elephas maximus. In: The IUCN Red List of Threatened Species 2008.

<www.iucnredlist.org>. [Accessed 01 July 2020]. www.iucnredlist.org (Accessed May 11 2009).

Edwards, K. L., M. A. Miller, K. Carlstead, and J. L. Brown. 2019. Relationships between housing and management factors and clinical health events in elephants in North American zoos. PLoS One 14(6):e0217774. doi: 10.1371/journal.pone.0217774 
Fuller, G., S. W. Margulis, and R. Santymire. 2011. The effectiveness of indigestible markers for identifying individual animal feces and their prevalence of use in North American zoos. 30(4):379-398. doi: 10.1002/zoo.20339

Harris, M., S. Sherwin, and S. Harris. 2008. The welfare, housing and husbandry of elephants in UK zoos: , University of Bristol.

IUCN. 2020. The IUCN Red List of Threatened Species. Version 2020.1. <http://www.iucnredlist.org>. [Accessed 01 July 2020]. <http://www.iucnredlist.org> (Accessed July 01 2020).

Lee, B. 2019. AZA Regional Studbook for Asian Elephant (Elephas maximus). Association of Zoos and Aquariums, Portland, OR.

MacDougall-Shackleton, S. A., F. Bonier, L. M. Romero, and I. T. Moore. 2019. Glucocorticoids and "Stress" Are Not Synonymous. Integrative Organismal Biology 1(1)doi: https://doi.org/10.1093/iob/obz017

Mason, G. J., and J. S. Veasey. 2010. What do population-level welfare indices suggest about the well-being of zoo elephants? Zoo Biology 29(2):256-273. doi: 10.1002/zoo.20303

McCormick, S. D., and L. M. Romero. 2017. Conservation Endocrinology. BioScience 67(5):429-442. doi: 10.1093/biosci/bix026 \%J BioScience

Meehan, C. L., J. N. Hogan, M. K. Bonaparte-Saller, and J. A. Mench. 2016a. Housing and Social Environments of African (Loxodonta africana) and Asian (Elephas maximus) Elephants in North American Zoos. PLoS One 11(7):e0146703. doi: 10.1371/journal.pone.0146703

Meehan, C. L., J. A. Mench, K. Carlstead, and J. N. Hogan. 2016b. Determining Connections between the Daily Lives of Zoo Elephants and Their Welfare: An Epidemiological Approach. PLoS One 11(7):e0158124. doi: 10.1371/journal.pone.0158124

Moberg, G. P. 2000. Biological response to stress: implications for animal welfare. CABI $\mathrm{Pu}$, New York, New York, USA.

Möstl, E., and R. Palme. 2002. Hormones as indicators of stress. Domestic Animal Endocrinology 23(1):67-74. doi: https://doi.org/10.1016/S0739-7240(02)00146-7 
Nelson, R. J. 2011. An introduction to Behavioral Endocrinology. Fourth ed. Sinauer Associates, Inc., Sunderland, Massachusetts.

Norkaew, T., J. L. Brown, P. Bansiddhi, C. Somgird, C. Thitaram, V. Punyapornwithaya, K. Punturee, P. Vongchan, N. Somboon, and J. Khonmee. 2018. Body condition and adrenal glucocorticoid activity affects metabolic marker and lipid profiles in captive female elephants in Thailand. PLoS One 13(10):e0204965. doi: https://doi.org/10.1371/journal.pone.0204965

Norkaew, T., J. L. Brown, P. Bansiddhi, C. Somgird, C. Thitaram, V. Punyapornwithaya, K. Punturee, P. Vongchan, N. Somboon, and J. Khonmee. 2019. Influence of season, tourist activities and camp management on body condition, testicular and adrenal steroids, lipid profiles, and metabolic status in captive Asian elephant bulls in Thailand. PLOS ONE 14(3):e0210537. doi:

10.1371/journal.pone.0210537

Othman, N., B. Goossens, C. P. I. Cheah, S. Nathan, R. Bumpus, and M. Ancrenaz. 2019. Shift of paradigm needed towards improving human-elephant coexistence in monoculture landscapes in Sabah. International Zoo Yearbook 53(1):161-173. doi: 10.1111/izy.12226

Palme, R. 2019. Non-invasive measurement of glucocorticoids: Advances and problems. Physiol Behav 199:229-243. doi: https://doi.org/10.1016/j.physbeh.2018.11.021

Palme, R., D. G. A. Meltzer, and S. K. Stead. 2000. The measurement of glucocorticoid concentrations in the serum and faeces of captive African elephants (Loxodonta africana) after ACTH stimulation : research communication. Journal of the South African Veterinary Association 71(3):192-196.

Proctor, C., and J. Brown. 2015. Influence of Handling Method on Adrenal Activity in Zoo African and Asian Elephants. J Zoo Aquar Res 3:1-1.

Ralph, C. R., and A. J. Tilbrook. 2016. INVITED REVIEW: The usefulness of measuring glucocorticoids for assessing animal welfare. J Anim Sci 94(2):457-470. doi: https://doi.org/10.2527/jas.2015-9645

Romero, L. M. 2004. Physiological stress in ecology: lessons from biomedical research. Trends Ecol Evol 19(5):249-255. doi: https://doi.org/10.1016/j.tree.2004.03.008

Scheer, F. A. J. L., T. J. Shea, M. F. Hilton, and S. A. Shea. 2008. An Endogenous Circadian Rhythm in Sleep Inertia Results in Greatest Cognitive Impairment upon Awakening during the Biological Night. Journal of Biological Rhythms 23(4):353-361. doi: 10.1177/0748730408318081 
Shepherdson, D., K. Carlstead, and N. Wielebnowski. 2004. Cross-institutional assessment of stress responses in zoo animals using longitudinal monitoring of faecal corticoids and behaviour. Anim Welf 13:S105-S114.

Sheriff, M. J., B. Dantzer, B. Delehanty, R. Palme, and R. Boonstra. 2011. Measuring stress in wildlife: techniques for quantifying glucocorticoids. Oecologia 166(4):869-887.

van Wees, M., and M. Damen. 2015. EAZA European Studbook for Asian Elephants (Elephas maximus).

Veasey, J. 2006. Concepts in the care and welfare of captive elephants. International Zoo Yearbook 40(1):63-79. doi: 10.1111/j.1748-1090.2006.00063.x

Vidya, T. N. C., and R. Sukumar. 2005. Social and reproductive behaviour in elephants. Current Science 89(7):1200-1207.

Watters, J. V., and N. Wielebnowski. 2009. Introduction to the special issue on zoo animal welfare. 28(6):501-506. doi: 10.1002/zoo.20287

Whitham, J. C., and N. Wielebnowski. 2013. New directions for zoo animal welfare science. Appl Anim Behav Sci 147(3):247-260. doi: https://doi.org/10.1016/j.applanim.2013.02.004

Wielebnowski, N. 2003. Stress and distress: evaluating their impact for the well-being of zoo animals. Journal of the American Veterinary Medical Association 223(7):973-977. doi: 10.2460/javma.2003.223.973

Wielebnowski, N. C., N. Fletchall, K. Carlstead, J. M. Busso, and J. L. Brown. 2002. Noninvasive assessment of adrenal activity associated with husbandry and behavioral factors in the North American clouded leopard population. Zoo Biol 21(1):77-98.

Williams, E., C. Chadwick, L. Yon, and L. Asher. 2018. A review of current indicators of welfare in captive elephants (Loxodonta africana and Elephas maximus). Anim Welf 27(3):235-249.

Withers, P. C. 1992. Comparitive Animal Physiology. Brooks/Cole - Thompson Learning, Pacific Grove. 
Zhdanova, I. V., K. Masuda, C. Quasarano-Kourkoulis, D. L. Rosene, R. J. Killiany, and S. Wang. 2011. Aging of Intrinsic Circadian Rhythms and Sleep in a Diurnal Nonhuman Primate, Macaca mulatta. Journal of Biological Rhythms 26(2):149159. doi: 10.1177/0748730410395849 
CHAPTER 2: Effects of physiological changes and social life events on adrenal glucocorticoid activity in female Asian elephants (Elephas maximus)

PUBLICATION STATUS: In press pending acceptance of revisions with the journal PLOS One.

Sharon S. Glaeser ${ }^{1,}$, Katie L. Edwards ${ }^{2,3}$, Nadja Wielebnowski ${ }^{1}$, Janine L. Brown ${ }^{2}$

1 Oregon Zoo, 4001 SW Canyon Rd, Portland, Oregon, 97221, United States of America 2 Center for Species Survival, Smithsonian Conservation Biology Institute, Smithsonian National Zoological Park, Front Royal, Virginia, United States of America, 3 North of England Zoological Society, Chester Zoo, Upton-by-Chester, United Kingdom

* Sharon.Glaeser@oregonzoo.org

Short title: Long-term variation in adrenal glucocorticoid activity in Asian elephants

Keywords: Asian Elephant, Elephas maximus, adrenal activity, adrenocortical activity, hormones, cortisol, glucocorticoids, progestagens, endocrinology, female reproduction, ovarian cyclicity, demographics, social, long-term variation, individual variation, hormone patterns, animal welfare 


\section{Abstract}

Ensuring good health and welfare is an increasingly important consideration for conservation of endangered species and includes breeding of individuals managed under human care. Understanding how factors in the captive environment affect individual animal wellbeing can be aided by long-term monitoring of biological functioning. This study involved longitudinal assessments (4 to 28 years) of reproductive and adrenal hormones in zoo-housed female Asian elephants (Elephas maximus) (age range 4 to 71 years). The goal was to elucidate patterns in adrenal glucocorticoid (GC) activity across the ovarian cycle and in association with reproductive and demographic factors, and examine individual response to major social changes. Concentrations of serum and urinary cortisol covaried more consistently with physiological changes (ovarian cycle phase, puberty, pregnancy, lactational anestrus, and age) than with social life events (births, deaths, and facility transfers). Cortisol fluctuated across the ovarian cycle with mean concentrations being higher in the follicular than in the luteal phase, but with differences between parous and nulliparous females. Individuals responded differently to social change, with some exhibiting increases, decreases, or no change in cortisol production in response to similar stimuli. Data suggest that social relationships, social support, temperament, and life history are important factors influencing the impact of perceived stressors on physiological measures. Furthermore, the differences in adrenal glucocorticoid (GC) responses to life events reinforce that welfare should be assessed on an individual basis. Outcomes from this study will help us gain a better understanding of the underlying conditions or states that can affect adrenal GC activity, and the variation 
we can expect with physiological change versus that in which the response is individualistic and event based. 


\section{Introduction}

Welfare of elephants, whether free-ranging or managed to varying degrees under human care, has become an increasingly important consideration for conservation and breeding efforts worldwide. Individuals with good health and welfare generally experience increased survivorship and reproduction and thus are better able to contribute to population sustainability. To assess welfare, it is important to measure indicators of physical, physiological and psychological states at both the individual and population level. Long-term monitoring helps establish baseline values for individuals, populations, and species, and allows for detection of patterns that are important to consider when evaluating responses to external stimuli. Hormone monitoring in particular can be used to assess physiological health, and is increasingly used to enhance reproduction and welfare of ex-situ and in-situ populations of various taxa [1]. Through longitudinal measures of hormones in elephants in western zoos, we have characterized basic endocrine function and determined how environmental factors (e.g., social structures, life events, climate, feeding strategy) affect gonadal and adrenal function $[2,3]$. We have used this information to improve captive management through better timing of breeding (both natural and assisted), providing more social opportunities, and improving exhibit design and husbandry to enhance elephant welfare.

Indicators of stress are commonly measured as welfare outcomes, with the most frequently used indicator being glucocorticoids (GCs) secreted from the adrenal cortex in response to a variety of stimuli [4-8], both positive (e.g., birth, excitement, mating) or 
negative (e.g., fear, anxiety, pain) [5, 9-11]. The primary role of GCs at basal levels is energy regulation, but higher concentrations facilitate physiological changes associated with the stress response [12], which is an adaptive reaction to real or perceived stressors resulting in a suite of behavioral, physiological and neuroendocrine changes to help an organism cope and re-establish homeostasis $[4,13,14]$. Short-term stressors (e.g., fighting, hunting, predator avoidance) cause an acute response; however, prolonged exposure to physical or psychological stressors (e.g., lack of nutrition, social aggression, poor husbandry) can result in chronic activation of the hypothalamic-pituitary-adrenal (HPA) axis with high concentrations of GCs that have deleterious effects on health and welfare including immunosuppression, decreased wound healing, increased susceptibility to disease, and poor reproduction $[13,15]$. In elephants, GCs have been found to increase in response to adverse environmental conditions and physiological stress, e.g., with reduced food and water availability $[16,17]$ and injury [18] in free-ranging African elephants; and low winter temperatures [19] and physical exertion [20] in captive Asian elephants.

Life events such as births, deaths, and facility transfers disrupt stable social groups by adding or removing individuals, and can influence physiology and behavior in elephants. Birth is generally considered a positive stimulus. Group dynamics and activities may change with focus on a calf [21], and non-maternal female elephants often participate in allomothering [22]. By contrast, loss of individuals and social bonds is generally considered a negative stimulus. Wild elephants have been observed showing empathetic behaviors towards dying and deceased conspecifics [23, 24], suggesting that death of 
conspecifics, in addition to disrupting social groups, can be emotionally distressing for individual elephants at least in the short-term. Similarly, disruption in group composition from poaching in wild female African elephants negatively affected reproduction and was associated with increased GCs [25]. Elephants have also shown GC increases with transportation and relocation [26-29], which can result in dissolution of social bonds or require the development of new relationships. For example, captive female elephants exhibited increased GCs and behavioral changes during the process of social introduction $[30,31]$. However, it is important to note that increases in GCs in elephants occur under normal physiological conditions; e.g., diurnally in a circadian rhythm (24 hour sleepwake cycle) with higher concentrations in the morning [32-35], seasonally, albeit not consistently in temperature-controlled captive environments [32, 36], across the estrous cycle in females [37] and during musth in males [38, 39], and in association with stage of gestation and parturition [17, 40, 41]. Increases in GCs can also indicate arousal [5]. Males in a multitude of species have shown increased GCs during rut and in response to mating stimuli [42-44] and acute exercise [45, 46].

This study involved longitudinal assessments (4 to 28 years) of reproductive and adrenal hormones in several female Asian elephants housed at two North American zoos with a longstanding history of hormone monitoring. This extensive data set allowed us to examine patterns in adrenal GC activity and differences in individual response to a number of social changes. We examined adrenal variation with reproductive state and across the estrous cycle in elephants of varying ages and parity, as well as in response to major life events such as births, deaths, and facility transfers in and out of herdmates. 
Outcomes from this study can help us gain a better understanding of underlying conditions affecting patterns in adrenal GC activity, and how social change may affect the welfare of Asian elephants.

\section{Materials and methods}

\section{$\underline{\text { Animals and sample collection }}$}

Female Asian elephants $(\mathrm{n}=11)$ were housed at two Association of Zoos and Aquariums (AZA) accredited-zoos: Oregon Zoo (OZ, $\mathrm{n}=3$, Elephas maximus indicus, $\mathrm{n}=1$ E. $m$. borneensis) and the Smithsonian National Zoological Park (NZP, $\mathrm{n}=5$ E. m. maximus, $\mathrm{n}=2$ E. $m$. indicus $)$. Elephants were both wild-born $(\mathrm{n}=8)$ and zoo-born $(\mathrm{n}=3)$; parous $(n=1)$, multiparous $(n=4)$ and nulliparous $(n=6)$; with an age range of 4 to $\sim 71$ years encompassing puberty through reproductive senescence (Table 1). Two females at NZP were periodically treated with a gonadotrophin releasing hormone $(\mathrm{GnRH})$ vaccine to shut down ovarian steroidogenic activity to resolve uterine pathologies. Details on the successful treatment of one female with Repro-BLOC ${ }^{\circledR}$ is provided by Boedeker et al. [47]; the other female also showed resolution of uterine cysts during treatment for $\sim 1$ year with Improvest (unpublished). This study was approved by the Welfare and Research Committees at the Oregon Zoo (OZ), and by the Institution Animal Care and Use Committees at the Smithsonian National Zoological Park (NZP). 
Table 1: Female elephants included in this study.

Housing facility, individual, origin, age range during study, parity, whether the individual exhibited normal ovarian cyclicity during the study, number of samples analyzed, and statistical analyses performed for each individual.

\begin{tabular}{|c|c|c|c|c|c|c|c|}
\hline Facility & $\begin{array}{c}\text { Animal } \\
\text { ID }\end{array}$ & Origin & $\begin{array}{c}\text { Age } \\
\text { range } \\
\text { (years) }\end{array}$ & Parity & $\begin{array}{l}\text { Exhibited } \\
\text { Normal } \\
\text { Cycling }\end{array}$ & $\begin{array}{c}\text { Sample } \\
\text { Number }\end{array}$ & $\begin{array}{c}\text { Effects } \\
\text { analyzed } \\
\text { using } \\
\text { GLMMs }\end{array}$ \\
\hline $\mathrm{OZ}^{\mathrm{a}}$ & $\mathrm{F} 1 \mathrm{OZ}$ & Zoo-born & $4-14$ & Parous $^{\mathrm{c}}$ & Yes & 338 & $\begin{array}{l}\text { RS, OC, Age, } \\
\text { D1, T1, T2 }\end{array}$ \\
\hline $\mathrm{OZ}$ & $\mathrm{F} 2 \mathrm{OZ}$ & Wild & $\sim 9-15$ & Nulliparous & Yes & 229 & $\begin{array}{l}\text { RS, OC, Age, } \\
\text { D1, T2 }\end{array}$ \\
\hline $\mathrm{OZ}$ & $\mathrm{F} 3 \mathrm{OZ}$ & Zoo-born & $11-26$ & Nulliparous & Yes & 348 & $\begin{array}{l}\text { OC, Age, D1, } \\
\text { D2, D3, D4, } \\
\text { T1, T2, T5 }\end{array}$ \\
\hline $\mathrm{OZ}$ & $\mathrm{F} 4 \mathrm{OZ}$ & Wild & $\sim 39-51$ & Multiparous & Yes & 488 & $\begin{array}{l}\text { OC, Age, D2, } \\
\text { D3, D4, T1, } \\
\text { T2, T5, DS1 }\end{array}$ \\
\hline $\mathrm{NZP}^{\mathrm{b}}$ & F5NZ & Wild & $\sim 15-43$ & Multiparous & Yes $^{\mathrm{d}}$ & 1230 & $\begin{array}{l}\text { RS, OC, Age, } \\
\text { D5, D6, T3, } \\
\text { T4, T6, TS1 }\end{array}$ \\
\hline $\mathrm{NZP}$ & F6NZ & Wild & $\sim 28-41$ & Nulliparous & Yes & 576 & $\begin{array}{l}\text { OC, Age, B1, } \\
\text { B2, D5, DS2 }\end{array}$ \\
\hline $\mathrm{NZP}$ & F7NZ & Wild & $\sim 46-71$ & Nulliparous & Yes $^{\mathrm{d}}$ & 1121 & $\begin{array}{l}\text { RS, OC, Age, } \\
\text { B2, D5, D6, } \\
\text { T3, T4, T6 }\end{array}$ \\
\hline NZP & F8NZ & Zoo-born & $24-28$ & Multiparous & Yes & 166 & OC, T6, TS3 \\
\hline $\mathrm{NZP}$ & F9NZ & Wild & $\sim 39-43$ & Multiparous & Yes & 174 & $\begin{array}{l}\text { RS, OC, T6, } \\
\text { TS2 }\end{array}$ \\
\hline NZP & F10NZ & Wild & $\sim 38-42$ & Nulliparous & No & 233 & not modelled \\
\hline $\mathrm{NZP}$ & F11NZ & Wild & $\sim 38-43$ & Nulliparous & No & 242 & not modelled \\
\hline
\end{tabular}

${ }^{\mathrm{a}} \mathrm{OZ}=$ Oregon $\mathrm{Zoo}$

${ }^{\mathrm{b}} \mathrm{NZP}=$ National Zoological Park

${ }^{\mathrm{b} B e c a m e}$ multiparous after data collection.

${ }^{\mathrm{c} N o t}$ cycling during treatment with a GnRH vaccine.

$\mathrm{RS}=$ Reproductive state

OC = Ovarian cycle phase (see Table 2)

Age $=$ Age analysis (see Table 3)

B\# = Birth to herdmate (see Table 4)

D\# = Death of herdmate (see Table 5)

$\mathrm{T} \#=$ Transfer of herdmate (see Table 6)

TS\# = Transfer of self (see Table 7)

DS\# = Death of self $($ see Table 8$)$ 
During the study period, females at OZ were housed as a single herd or as two separate herds comprising two to four individuals each. Two to four adult bulls were present at any given time and were housed separately from each other and from females, except for breeding or for male/female socialization. Females at NZP were housed as a single herd of three initially, and then as two separate herds comprising two to five individuals after the arrival of three new elephants. One juvenile male was gradually housed separately starting around the age of 8 , but occasionally was put with females for socialization until he was transferred out at age 14. An adult bull (36 years of age) was integrated into the herd at NZP in the last year of the study.

Most elephants (all for OZ; 6 of 7 for NZP) allowed blood collection (without sedation) as part of their normal management routine. Blood was collected $(3-9 \mathrm{ml})$ into red top serum separator tubes from an ear or leg vein by elephant care staff. Blood was maintained at $\sim 4^{\circ} \mathrm{C}$, and then centrifuged at $1500 \mathrm{~g}$ within a few hours of collection to separate serum. During certain periods, five elephants at NZP were not reliable for blood collection, so urine was collected by midstream catch in a cup or tube, or off the enclosure floor, and then centrifuged at $500 \mathrm{~g}$ to remove debris within $\sim 2$ hours of collection. Serum and urine samples were stored at $\sim 20^{\circ} \mathrm{C}$ or colder until analysis. Samples were collected weekly, and in the morning to control for diurnal patterns of cortisol secretion [32]. 


\section{$\underline{\text { Immunoassays }}$}

Approximately weekly samples were analyzed routinely for progestagens and cortisol to assess reproductive and adrenal GC activity throughout the study period. Data from 1995 to 2009 was used for OZ females, and data from 1991 to 2019 for NZP females.

Cortisol concentrations in serum samples collected through 2014 were measured using a solid-phase cortisol ${ }^{125}$ I radioimmunoassay (RIA) (Siemens Healthcare Diagnostics Inc., Terrytown, NY, USA) following the methods of Brown et al. [38]. This assay was discontinued at the end of the 2014 , after which serum cortisol was analyzed using a solid-phase cortisol ${ }^{125}$ I RIA (CortiCote, MP Biomedicals, Santa Ana, CA; catalog \# 06B256440) with modifications described in Edwards et al. [48]. Measured values did not differ between the two assays.

Urinary cortisol concentrations were quantified by a double-antibody enzymeimmunoassay (EIA) adapted from Brown et al. [32] and modified by Edwards et al. [48]. It has been previously demonstrated in elephants that circulating cortisol is excreted in the urine in its native form as free cortisol $[32,49]$. The EIA utilized a polyclonal rabbit anti-cortisol antibody (R4866; C.J. Munro, University of California, Davis) and horseradish peroxidase (HRP)-conjugated cortisol label (C.J. Munro, University of California-Davis, Davis, CA). Microtiter plates were pre-coated with secondary goat-anti rabbit IgG antibody (A009, Arbor Assays, Ann Arbor, MI) described by Edwards et al. [48]. Cortisol standards (50 $\mu \mathrm{l} ; 0.078-20 \mathrm{ng} / \mathrm{ml})$, controls (50 $\mu \mathrm{l})$, and samples $\left(50 \mu \mathrm{l}\right.$; diluted 1:20 to 1:100 in phosphate buffer [0.039M NaH $\mathrm{PO}_{4}, 0.061 \mathrm{M}$ 
$\left.\left.\mathrm{Na}_{2} \mathrm{HPO}_{4}, 0.15 \mathrm{M} \mathrm{NaCl} ; \mathrm{pH} 7.0\right]\right)$ were added to wells in duplicate, followed by cortisolHRP (25 $\mu 1 ; 1: 15$ 000; C. Munro, University of California, Davis, CA) to all wells. The primary anti-cortisol antibody $(25 \mu \mathrm{l}$; R4866; 1:60 000) was added to all wells except non-specific binding (NSB) wells, followed by incubation for 1 hour at room temperature (RT). Unbound components were removed by washing five times with buffer (X007, Arbor Assays), followed immediately with addition of a chromogen solution containing TMB (100 $\mu 1, \mathrm{X} 019$, Arbor Assays) to each well. After incubation for $5 \mathrm{~min}$ at RT, the reaction was halted by adding stop solution (50 $\mu$; X020 Arbor Assays), and optical densities were determined at $450 \mathrm{~nm}$ with a reference of $630 \mathrm{~nm}$. Steroid crossreactivities of the R4866 antibody were previously reported in Young et al. [50]. Cross reactivities for the Healthcare Diagnostics cortisol RIA antibody were as follows: cortisol $100 \%$, prednisolone $76 \%$, methylprednisolone $12 \%$, 11-deoxycortisol $11.4 \%$, prednisone $2.3 \%$, betamethasone $1.6 \%$, cortisone $0.98 \%$, and corticosterone $0.94 \%$. Cross reactivities for the MP Biomedicals cortisol RIA antibody are as follows: cortisol 100.0\%, prednisolone $94.1 \%, 11$-deoxycortisol $2.2 \%$, prednisone $1.2 \%$, corticosterone $1.2 \%$, cortisone $0.8 \%$, dexamethasone $0.8 \%, 17$-hydroxyprogesterone $<0.05 \%$, and metyrapone $<0.01 \%$.

Progestagens in serum samples collected through 2014 were measured using a solidphase ${ }^{125}$ I progesterone RIA (Siemens Healthcare Diagnostics Inc.) validated for elephants $[2,41,51]$. After this assay was discontinued, serum samples from 2015 to 2019 and all urine samples were analyzed using a double-antibody progesterone EIA modified from the single-antibody assay of Brown et al. [52]. Anti-mouse antibody 
(A008, Arbor Assays, Ann Arbor, MI) in coating buffer (X108, Arbor Assays) (150 $\mu$ at $10 \mu \mathrm{g} / \mathrm{ml}$ ) was added to 96-well microtiter plates (Costar, Corning Life Sciences,

Tewkesbury, MA) followed by incubation at RT for 15-24 hours. Unbound antibody was then washed from wells with buffer (X007, Arbor Assays). Blocking solution (250 $\mu$; X109, Arbor Assays) was added to each well and incubated for 4 to 24 hours at RT. Blocking solution was then removed and plates were dried at RT in a desiccator cabinet, packaged in vacuum-sealed bags and stored at $4{ }^{\circ} \mathrm{C}$ until use. Progesterone standards (50 $\mu \mathrm{l} ; 0.016-4.0 \mathrm{ng} / \mathrm{ml}$; P0130; Sigma Chemical Co.), controls (50 $\mu \mathrm{l})$, and samples (50 $\mu \mathrm{l}$; neat [serum]; diluted 1:20 to 1:100 [urine] in phosphate buffer [0.039M NaH $\mathrm{PO}_{4}$, 0.061M Na $\left.2 \mathrm{HPO}_{4}, 0.15 \mathrm{M} \mathrm{NaCl} ; \mathrm{pH} 7.0\right]$ ) were added to wells in duplicate, followed by progesterone-HRP (25 $\mu \mathrm{l} ; 1: 90,000$; C. Munro, University of California, Davis, CA) to all wells. The monoclonal anti-progesterone antibody (25 $\mu$; CL425 1:50,000) was added to all wells except NSB wells, followed by incubation for 2 hours at RT. Unbound components were removed by washing five times with buffer (X007, Arbor Assays), followed immediately by adding a chromogen solution containing TMB (100 $\mu 1$, X019, Arbor Assays) to each well. After incubation for $30 \mathrm{~min}$ at RT, the reaction was halted by adding stop solution (50 $\mu \mathrm{l}$; X020 Arbor Assays), and optical densities were determined at $450 \mathrm{~nm}$ with a reference of $630 \mathrm{~nm}$. Steroid cross-reactivities of the CL425 antibody were previously reported in Rolland et al. [53]. Cross reactivities for the Healthcare Diagnostics progesterone RIA were as follows: progesterone $100 \%, 5 \alpha$-pregnan-3,20dione $9 \%, 17 \alpha$-hydroxyprogesterone $3.4 \%, 5 \beta$-pregnan-3,20-dione 3.2\%, 11-deoxycorticosterone $2.2 \%$, corticosterone $0.9 \%$, medroxyprogesterone $0.3 \%, 20 \alpha$ dihydroprogesterone $0.2 \%$, pregnenolone $0.1 \%$, and testosterone $0.1 \%$. 
Cortisol assay sensitivities were $2.5 \mathrm{ng} / \mathrm{ml}$ for the RIA and $0.08 \mathrm{ng} / \mathrm{ml}$ for the EIA. Progesterone assay sensitivities were $0.05 \mathrm{ng} / \mathrm{ml}$ for the RIA and $0.02 \mathrm{ng} / \mathrm{ml}$ for the EIA. All serum and urine samples were analyzed unextracted. Urinary steroids were indexed by creatinine (Cr) concentration according to Monfort et al. [54]. All assays had been validated for elephants by demonstrating: (1) parallelism between dilutions of pooled serum samples to the respective standard curve preparation and (2) significant (>90\%) recovery of exogenous standard hormone added to pooled samples before analysis. The inter- and intra-assay coefficients of variation (CVs) were maintained below 15\% and $10 \%$, respectively, for all assays and sample types.

Determination of reproductive state and ovarian cycle phase

Reproductive state (prepubertal, normal cycling, pregnant, lactational anestrus, irregular cycling, contracepted, acyclic) and ovarian cycle phase (luteal, follicular) for female elephants at $\mathrm{OZ}$ were previously determined by Glaeser $e t$ al. [55] for female elephants at OZ, and the methods applied to analyzing reproductive status of the NZP elephants. First, baseline hormone concentrations were calculated for each individual using an established iterative process [51] conducted in $\mathrm{R}$ version 3.5.2 [56] using the package hormLong [57]. For each individual, all data points with values that exceeded the mean plus 1.5 standard deviations (SD) were removed and the process repeated until all values exceeding the mean $+1.5 * \mathrm{SD}$ had been removed. The remaining data points defined the baseline for that individual, and the baseline cut-off was the highest value that remained after this iterative process. 
Ovarian cycles were determined based on progestagen patterns $[55,58,59]$ as follows:

(1) the onset of the luteal phase was defined as the first sample where progestogen concentration exceeded the baseline and remained elevated for at least two consecutive weeks and with a duration of at least four weeks; 2) the onset of the follicular phase was defined as the first sample where progestogen concentration fell below the baseline and remained below the baseline for at least two consecutive weeks; 3) single point fluctuations above or below baseline were considered within the same phase as the surrounding points; 4) data points on the baseline were included in the previous phase; 5) when data were not available for a given week, and that week appeared to coincide with the start or end of a luteal phase, it was added to the luteal phase. Ovarian cycle duration was calculated as the number of weeks from the first luteal phase sample through the last follicular phase sample. Cycles with durations outside $\pm 2 * \operatorname{SD}$ of the group mean were defined as outliers [55]. Acyclicity was defined as progestagen concentrations at baseline for extended periods encompassing the timeframe of multiple estrous cycles [51].

Descriptive statistics of ovarian cycle and phase durations were calculated using Excel (Microsoft ${ }$ Office Excel 2016; Microsoft, Corp., Redmond, WA, USA) for each individual, for all females combined, and for all females combined but with outliers among the group removed. 


\section{Demographics and life events}

Origin of birth, birth date, housing facility, parity, and life event data of births, deaths, and facility transfers (change in physical location) were obtained from the AZA Asian Elephant Regional Studbook [60]. During this study (1995-2009 for OZ, 1991-2019 for NZP), three elephants were born, six died, and 10 were transferred in or out of the two facilities. These data constituted social life events (events involving herdmates) and events that an individual physically experiences (transfer of themselves between facilities, birth of offspring, and their own health decline leading to euthanasia).

\section{Data analysis}

Median, range, mean, standard deviation (SD), and coefficient of variation (CV) in serum and urinary cortisol concentrations were calculated for each individual and all elephants combined, across all reproductive states, and during periods limited to normal cycling. All descriptive statistics were calculated using Excel (Microsoft® Office Excel 2016; Microsoft, Corp., Redmond, WA, USA). Coefficients of variation (CV) in cortisol concentration were calculated for reproductive states and ovarian cycle phases. Differences between CVs were determined by the Brown-Forsythe Test [61, 62], run in $\mathrm{R}$ version 3.6.1 [56] using the package "onewaytests" [63]. Significance was assessed at the 0.05 level for all analyses.

Hormone data were analyzed using generalized linear mixed models (GLMMs) in MLwiN version 2.02 [64] to investigate differences in mean cortisol concentrations according to reproductive state (prepubertal, normal cycling, pregnant, lactational 
anestrus, irregular cycling, contracepted, acyclic), ovarian cycle phase (luteal, follicular), age, group demographics, and in response to major life events (births, deaths, transfers). GLMMs allow random effects to be incorporated into the model $[65,66]$ to control for non-independence of data, which in this study involved repeated serum or urine samples per subject. Kolmogorov-Smirnov normality tests and examination of plots showed distributions of hormone data were non-normal, so hormone data were $\log 10$ transformed to improve the distribution for the GLMMs.

Separate models were created for each elephant to investigate the effect of physiological and social changes on an individual-level, and with all elephants combined to investigate group-level effects. GLMMs in this study were based on models by Edwards et al. [67] and [68]. The dependent variable (hormone data), random effects (date of sample collection), and fixed effects were incorporated into each model. Reproductive state, ovarian cycle phase, life events, demographics (parity, origin, housing facility), and 10year age categories were fitted individually as categorical fixed effects; age was fitted as a continuous fixed effect. Only females that exhibited normal ovarian cycles at some point during the study were modelled. Reproductive state was added as an effect only in females that exhibited a change in state during the study, with cycling as the reference category in all models. Age was added as an effect only in females with at least 5 years of cortisol data, and cortisol changes with age were modelled across all reproductive states and also limited to periods of normal ovarian cycles to remove reproductive state as a confounding variable. Demographic effects were limited to periods of normal cycling for comparison. 
Life events were classified as pre- and post-event, with an equal duration of 30 days before and 30 days after the event (total 60 days) to assess adrenal GC response in a biologically relevant timeframe found in other studies [26, 29, 69, 70]. A second model for each event categorized the post-event into 15-day time periods to measure more acute responses, and included a third 15-day period to determine if concentrations returned to pre-event levels within 45 days. Two exceptions to this timeframe were transfers in of a herdmate (120 days pre- and 120 days post-event to account for an acclimation period of quarantine, introduction, and integration), and period of decline leading to euthanasia (final 30 days of life compared to the preceding 30 days; total 60 days). The day the event occurred was included in the post-event time period. All events experienced by individuals in this study were modelled with the exception of events having insufficient hormone data, and parturition events because they were confounded with a change in reproductive state.

The models were first run to determine differences in hormone concentration across reproductive states and between cycle phases. For females in which reproductive state or cycle phase had a significant effect, these effects were taken into account by inclusion as covariates in the models for events. For females in which reproductive state or cycle phase did not have a significant effect, these effects were not included in the models for events with the assumption that variation within the timeframe of the event was unrelated to reproductive state changes. 
A Normal error structure was used for all models. The significance of each fixed effect compared to the reference category was determined using the Wald statistic and chisquared $\left(\chi^{2}\right)$ distribution, with alpha set to 0.05 ; and significance of pair-wise comparisons was determined using $\chi^{2}$ with alpha set to 0.05 . A post-hoc power analysis was conducted on all models with a significant or close to significant effect to determine the minimum number of samples required to test the effect of cycle phase and life events on hormone concentration while keeping the power above the $80 \%$ threshold. Predicted means and standard errors (SE) of $\log 10$ cortisol and $\log 10$ GCM concentrations were back transformed to generate charts (Figs 1-5).

\section{Results}

Estrous cycle characteristics from female Asian elephants housed at $\mathrm{OZ}$ are described in Glaeser et al. [55]. An additional 188 estrous cycles were analyzed from five female Asian elephants at NZP (JLB, SG, unpublished). With all female elephants combined, an age range of 6 to 71 years, and a larger number of estrous cycles $(n=367)$, durations were found to have a greater range than previously reported [55, 71]. Estrous cycle duration ranged from 9 to 19 weeks (mean 14.5, SD 2.2 weeks), luteal phase from 4 to 17 weeks (mean 9.4, SD 2.0 weeks), and follicular phase from 2 to 15 weeks (mean 5.1, SD 2.0 weeks), with outliers for cycle duration among the group (OZ and NZP combined) removed. The percentage of outlier cycles was low $(2.7 \%)$, with five individuals exhibiting a total of 10 outlier cycles among the OZ and NZP elephants combined. F4OZ exhibited outliers after transfer out of a herdmate and the last cycle prior to euthanasia [55]. F6NZ and F7NZ exhibited outliers after birth to a herdmate, and F9NZ after a male 
subadult was transferred out. Variability in estrous cycle duration was considerably less than the variability in luteal and follicular durations for all females combined (estrous CV $=15 \%$, luteal $\mathrm{CV}=21 \%$, follicular $\mathrm{CV}=38 \%$ ).

\section{$\underline{\text { Long-term cortisol concentrations }}$}

Cortisol concentrations were highly variable among females (see Appendix A: S1 Tables A-D). For all elephants combined $(\mathrm{n}=11)$ and across all reproductive states, serum cortisol concentrations ( $\mathrm{N}=3840$ samples, $\mathrm{n}=7$ elephants) had a mean $(\mathrm{SD})$ of 16.07 (11.90) $\mathrm{ng} / \mathrm{ml}$ with a median (range) of $12.07(2.50-96.00) \mathrm{ng} / \mathrm{ml}$. For elephants or time periods when only urine was available, urinary cortisol concentrations $(\mathrm{N}=1305$ samples, $\mathrm{n}=6$ elephants) had a mean (SD) of 304.38 (299.85) $\mathrm{ng} / \mathrm{mg} \mathrm{Cr}$ with a median (range) of $223.08(0.08-3830.12) \mathrm{ng} / \mathrm{mg}$ Cr. Variability in cortisol across individuals, as determined by the $\mathrm{CV}$, ranged from $46.8 \%$ to $84.3 \%$ in serum; and $39.4 \%$ to $91.3 \%$ in urine.

For all females that exhibited normal ovarian cycling combined $(n=9)$, serum cortisol concentrations $(\mathrm{N}=2989, \mathrm{n}=7)$ resulted in the following means $(\mathrm{SD})$ and medians (range): mean $15.64(11.32) \mathrm{ng} / \mathrm{ml}$ and median $12.37(2.50-96.00) \mathrm{ng} / \mathrm{ml}$ across the cycle; mean $14.94(10.88) \mathrm{ng} / \mathrm{ml}$ and median $11.84(2.50-79.30) \mathrm{ng} / \mathrm{ml}$ during the luteal phase; and mean $16.88(11.66) \mathrm{ng} / \mathrm{ml}$ and median $13.70(2.50$ - 96.00) $\mathrm{ng} / \mathrm{ml}$ during the follicular phase. Urinary cortisol concentrations $(\mathrm{N}=504, \mathrm{n}=3)$ resulted in the following means (SD) and medians (range): mean 239.57 (179.22) ng/mg Cr and median 197.55 (0.08 - 1409.60) ng/mg Cr across the cycle; mean 216.22 (163.30) ng/mg Cr and median 
186.26 (0.08 - 1409.60) ng/mg Cr during the luteal phase; and mean 276.56 (181.60) $\mathrm{ng} / \mathrm{mg} \mathrm{Cr}$ and median 242.18 (35.23 - 1000.00) ng/mg Cr during the follicular phase.

\section{$\underline{\text { Reproductive state and adrenal GC activity }}$}

Reproductive state (prepubertal, normal cycling, pregnant, lactational anestrus, irregular cycling, contracepted, acyclic) was a significant predictor of mean cortisol concentration in four of the five individuals who changed state at least once during the study, although the pattern varied among individuals. Two females experienced four reproductive states each, and three females experienced two reproductive states each.

In two females that started cycling during the study, female F2OZ (Fig 1C) had higher mean serum cortisol concentrations $\left(\mathrm{GLMM}\right.$ coefficient $=0.130, \mathrm{SE}=0.044, \chi^{2}=8.699$, $\mathrm{df}=1, \mathrm{p}=0.003$ ), but lower variability (Appendix A: S2 Table A) in the prepubertal state compared to the cycling state; whereas female F1OZ (Fig 1A) exhibited lower mean serum cortisol concentration $\left(\mathrm{GLMM}\right.$ coefficient $=-0.127, \mathrm{SE}=0.051, \chi^{2}=6.284$, $\mathrm{df}=1, \mathrm{p}=0.012$ ) and variability (Appendix A: S2 Table A) in the prepubertal state.

In two females that gave birth, serum cortisol concentrations were highest during lactational anestrus. In female F1OZ (Fig 1A) there was a significant difference between all states, with mean cortisol concentrations being higher during lactational anestrus compared to pregnancy $\left(\chi^{2}=13.24, \mathrm{df}=1, \mathrm{p}<0.001\right)$, during pregnancy compared to cycling $\left(\mathrm{GLMM}\right.$ coefficient $\left.=0.134, \mathrm{SE}=0.040, \chi^{2}=11.24, \mathrm{df}=1, \mathrm{p}=0.001\right)$, and during cycling compared to the prepubertal state $($ GLMM coefficient $=-0.127$, 
$\left.\mathrm{SE}=0.051, \chi^{2}=6.284, \mathrm{df}=1, \mathrm{p}=0.012\right)$. Variability in cortisol concentrations followed the same pattern as the mean, with the $\mathrm{CV}$ being highest during lactational anestrus, followed by pregnancy, then cycling, and lowest in the prepubertal state (Appendix A: S2 Table A) In female F5NZ (Fig 1B), mean cortisol concentrations were higher during lactational anestrus compared to pregnancy $\left(\chi^{2}=6.411, \mathrm{df}=1, \mathrm{p}=0.011\right)$, cycling $\left(\mathrm{GLMM}\right.$ coefficient $\left.=0.172, \mathrm{SE}=0.054, \chi^{2}=10.827, \mathrm{df}=1, \mathrm{p}=0.001\right)$, and contracepted $\left(\chi^{2}=14.043, \mathrm{df}=1, \mathrm{p}=0.002\right)$ states. There was no difference between pregnant and cycling states $(\mathrm{p}=0.399)$. In contrast to $\mathrm{F} 1 \mathrm{OZ}$, variability in cortisol in F5NZ was lowest in lactational anestrus with no significant difference in CV across other reproductive states (Appendix A: S2 Table A). Female F5NZ gave birth to two calves during the study, and her mean serum cortisol concentrations and variability were lower with the second calf (male) compared to the first calf (female) during pregnancy (first calf: mean $=16.3 \mathrm{ng} / \mathrm{ml}, \mathrm{CV}=55.8 \%$; second calf: mean $=13.5 \mathrm{ng} / \mathrm{ml}, \mathrm{CV}=51.2 \%)$ and lactational anestrus (first calf: mean $=26.1 \mathrm{ng} / \mathrm{ml}, \mathrm{CV}=49.8 \%$; second calf: mean $=18.6$ $\mathrm{ng} / \mathrm{ml}, \mathrm{CV}=39.4 \%)$.

One female, F9NZ (Fig 1D), exhibited a period of irregular cycling [characterized by a either a long follicular phase (mean $+7 \mathrm{SD}$ ) or a luteal phase with concentrations close to baseline], and mean cortisol was lower during irregular cycling compared to normal cycling $\left(\right.$ GLMM coefficient $\left.=-0.209, \mathrm{SE}=0.062, \chi^{2}=11.45, \mathrm{df}=1, \mathrm{p}<0.001\right)$; variability also was lower during irregular cycling (Appendix A: S2 Table A). 
In two females that were contracepted to resolve uterine pathologies, lower mean serum cortisol concentrations during the contracepted state compared to cycling trended toward significance (F5NZ: GLMM coefficient $=-0.069, \mathrm{SE}=0.039, \chi^{2}=3.145, \mathrm{df}=1$, $\mathrm{p}=0.076$; F7NZ: GLMM coefficient $\left.=-0.034, \mathrm{SE}=0.017, \chi^{2}=3.790, \mathrm{df}=1, \mathrm{p}=0.052\right)$, but not when urine was analyzed (F5NZ: GLMM coefficient $=-0.040, \mathrm{SE}=0.049, \chi^{2}=$ 0.687, $\mathrm{df}=1, \mathrm{p}=0.407)$. Female F5NZ resumed normal cycling approximately 1 year after the last GnRH booster; whereas female F7NZ was contracepted at age 59 and never resumed cycling again even after the GnRH antibody titers returned to baseline. 


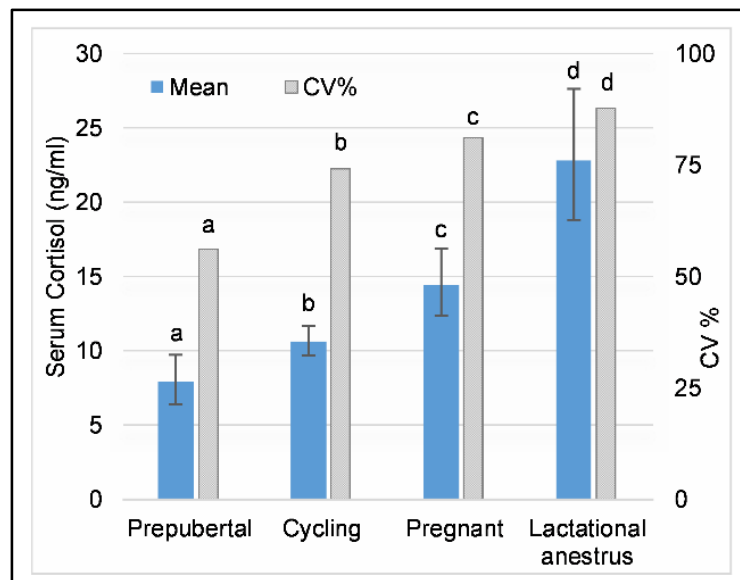

(A)

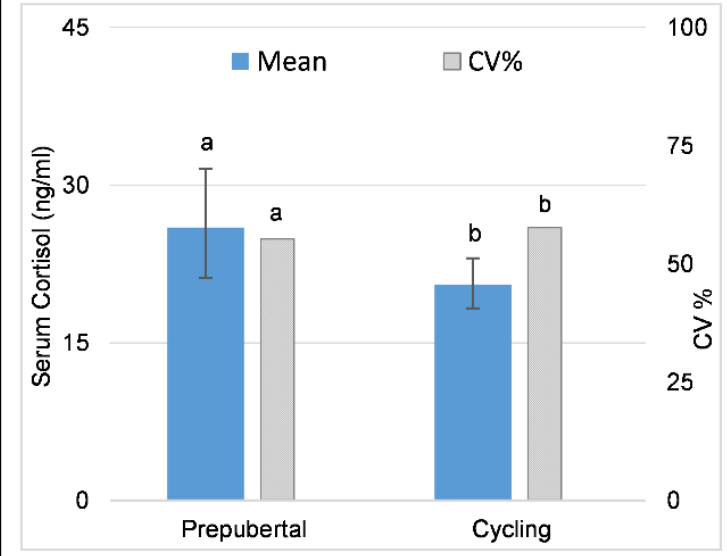

(C)

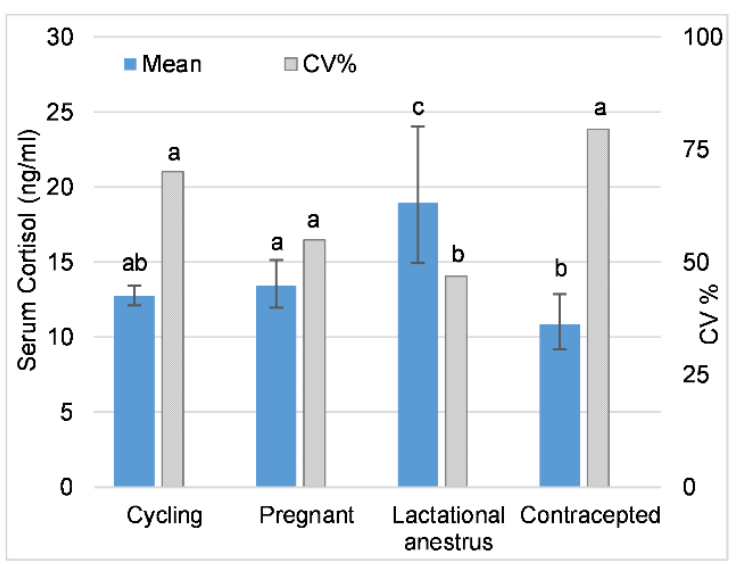

(B)

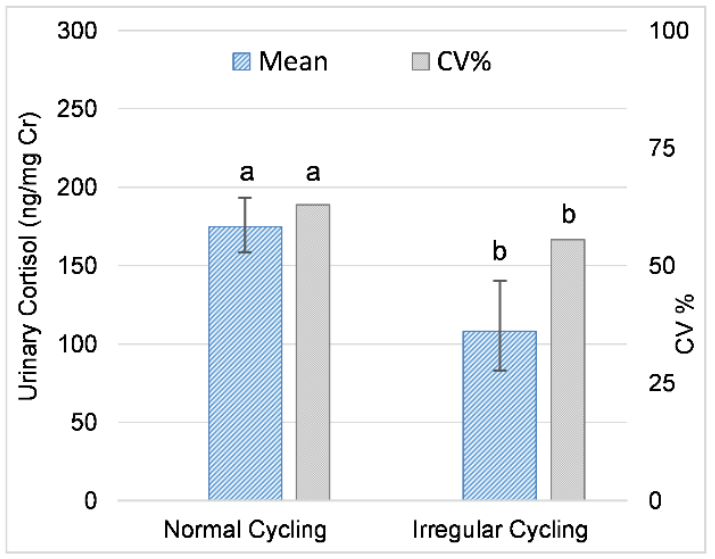

(D)

Fig 1: Reproductive state as a predictor of adrenal GC activity.

Predictions from GLMMs for serum and urinary cortisol concentration comparing mean values across reproductive states (error bars represent the standard error of the prediction). Letters denote significant differences in mean values and in coefficients of variation (CVs) across reproductive states. (A) F1OZ: mean cortisol in prepubertal < cycling < pregnant < lactational anestrus. (B) F5NZ: mean cortisol in cycling, pregnant, contracepted < lactational anestrus. (C) F2OZ: mean cortisol in cycling < prepubertal. (D) F9NZ: mean cortisol in irregular cycling < normal cycling.

\section{$\underline{\text { Ovarian cycle phase and adrenal GC activity }}$}

In females that exhibited normal ovarian cycles $(n=9)$, cycle phase was a significant predictor of mean cortisol in all females combined and in four individuals (Table 2), with lower concentrations during the luteal compared to the follicular phase (Fig 2). There was no significant difference in mean cortisol between cycle phases in five individuals; no 
females showed higher cortisol during the luteal phase. Post-hoc power analysis indicated that the number of samples was greater than the minimum required to achieve $80 \%$ power in all cases except two, where the power was reduced to $75 \%$ and $70 \%$. Variability in cortisol concentrations was higher during the luteal phase than the follicular phase for all elephants combined. Only those females with a statistical difference in mean cortisol concentrations between cycle phases also exhibited a statistical difference in variability, although the patterns varied (Appendix A: S2 Table B).

Table 2: Ovarian cycle phase as a predictor of adrenal GC activity.

Individual, sample type, effect size with standard error (SE), Wald statistic, and p-value from GLMMs, and whether the mean and coefficient of variation (CV) in cortisol in the luteal phase was higher or lower than in the follicular phase. Degrees of freedom (df) was 1 in all pair-wise comparisons.

\begin{tabular}{|l|c|c|c|c|c|c|}
\hline \multirow{2}{*}{ Individual(s) } & $\begin{array}{c}\text { Sample } \\
\text { Type }\end{array}$ & Effect Size (SE) & Wald & $\mathbf{P}$ & \multicolumn{2}{|c|}{$\begin{array}{c}\text { Luteal Phase Relative to } \\
\text { Follicular Phase }\end{array}$} \\
\cline { 5 - 7 } & & & & & Mean & CV \\
\hline All elephants & Serum & $-0.059(0.011)$ & 27.559 & $<\mathbf{0 . 0 0 1}$ & Lower & Higher \\
\hline $\begin{array}{l}\text { All elephants } \\
\text { all parous })\end{array}$ & Urine & $-0.138(0.033)$ & 17.390 & $<\mathbf{0 . 0 0 1}$ & Lower & Higher \\
\hline $\begin{array}{l}\text { Multiparous } \\
\text { females }\end{array}$ & Serum & $-0.078(0.018)$ & 18.930 & $<\mathbf{0 . 0 0 1}$ & Lower & Higher \\
\hline $\begin{array}{l}\text { Nulliparous } \\
\text { females }\end{array}$ & Serum & $-0.041(0.013)$ & 9.318 & $\mathbf{0 . 0 0 2}$ & Lower & Higher \\
\hline F1OZ & Serum & $-0.098(0.040)$ & 6.126 & $\mathbf{0 . 0 1 3}$ & Lower & Lower \\
\hline F5NZ & Serum & $-0.121(0.025)$ & 23.363 & $\mathbf{0 . 0 0 1}$ & Lower & Higher \\
\cline { 2 - 7 } & Urine & $-0.098(0.037)$ & 6.964 & $\mathbf{0 . 0 0 8}$ & Lower & Lower \\
\hline F7NZ & Serum & $-0.082(0.021)$ & 15.132 & $<\mathbf{0 . 0 0 1}$ & Lower & Lower \\
\hline F8NZ & Urine & $-0.229(0.065)$ & 12.438 & $<\mathbf{0 . 0 0 1}$ & Lower & Higher \\
\hline F3OZ & Serum & $-0.066(0.034)$ & 3.678 & 0.055 & -- & -- \\
\hline F2OZ & Serum & $0.054(0.055)$ & 0.977 & 0.323 & -- & -- \\
\hline F4OZ & Serum & $0.000(0.031)$ & -- & 1.000 & -- & - \\
\hline F6NZ & Serum & $-0.009(0.019)$ & 0.212 & 0.645 & -- & -- \\
\hline F9NZ & Urine & $-0.033(0.048)$ & 0.472 & 0.492 & -- & - \\
\hline
\end{tabular}

-- No significant difference

${ }^{\text {a }}$ Power $=70 \%$

${ }^{\mathrm{b}}$ Power $=75 \%$ 


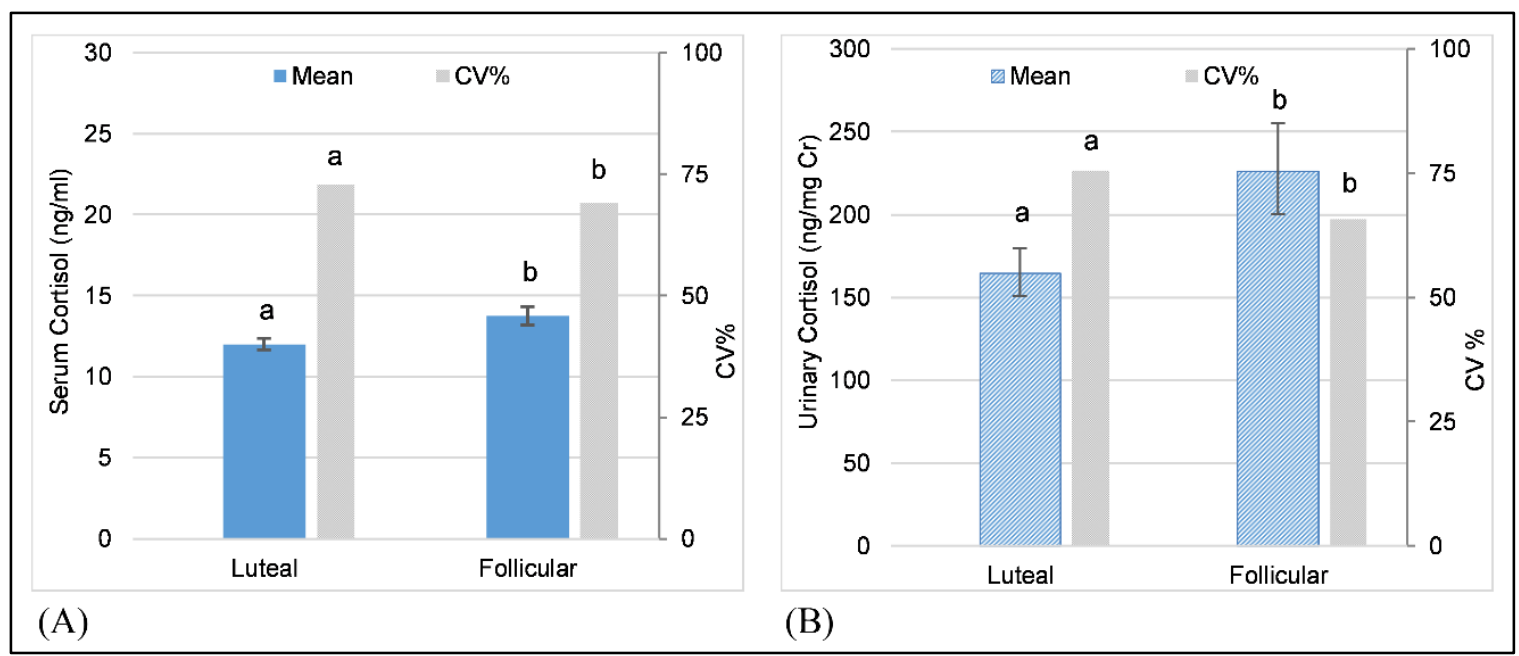

Fig 2: Ovarian cycle phase as a predictor of adrenal GC activity.

Predictions from GLMMs for cortisol concentrations comparing mean values between the luteal phase and follicular phase of the ovarian cycle for all elephants combined (error bars represent the standard error of the prediction). Letters denote significant differences in mean values and in coefficients of variation (CVs) between cycle phases. (A) Serum cortisol in all elephants combined. (B) Urinary cortisol in all elephants combined.

With regards to parity, cycle phase was a significant predictor of mean serum cortisol for parous and multiparous females combined $(\mathrm{n}=5)$ and nulliparous females combined $(\mathrm{n}=$ 4), with lower concentration during the luteal phase compared to the follicular phase in both groups (Table 2), and higher variability during the luteal compared the follicular phase (Table 2). Although these patterns were the same for both groups, the interaction of parity and cycle phase was significant $\left(\mathrm{GLMM}\right.$ coefficient $=0.039, \mathrm{SE}=0.015, \chi^{2}=$ $6.84, \mathrm{df}=1, \mathrm{p}=0.009$ ), with cycle phase having a larger effect on mean cortisol in parous and multiparous females than in nulliparous females. Furthermore, among parous and multiparous females, three females exhibited lower mean cortisol concentrations in the luteal phase and two exhibited no significant difference; among nulliparous females, one exhibited lower mean cortisol concentrations in the luteal phase and three exhibited no significant difference (Table 2). 


\section{Demographics factors and adrenal GC activity}

In normal cycling females, mean serum cortisol concentrations $(\mathrm{N}=2989$ samples, $\mathrm{n}=7$ elephants) did not differ between wild-born $(n=5)$ and zoo-born $(n=2)$ females (GLMM coefficient $\left.=-0.097, \mathrm{SE}=0.076, \chi^{2}=1.627, \mathrm{df}=1, \mathrm{p}=0.202\right)$, or between housing facilities (OZ: $\mathrm{n}=4$, NZP: $\mathrm{n}=3)\left(\mathrm{GLMM}\right.$ coefficient $=-0.045, \mathrm{SE}=0.075, \chi^{2}=0.362$, $\mathrm{df}=1, \mathrm{p}=0.547)$. Urinary cortisol could not be similarly compared because all females ( $\mathrm{n}$ =3) were at one facility and only one was zoo-born.

For females combined across all reproductive states $(n=7$ elephants with at least 5 years of cortisol data), there was no overall change in mean cortisol concentration with age, although differences were observed across the 10-year age categories (Table 3). Mean cortisol was lowest in the $0-10$ years age category, remained higher in the age categories spanning 11-60 years with 41-60 years being highest, and then decreased in the oldest age category (>61 years of age) (Fig 3A).

For females combined and limited to the state of normal cycling $(n=7)$, mean cortisol concentrations increased with age overall, and also across age categories (Table 3). Concentrations were lowest in the 0-10-year age category, higher in the 11-20 and 21-30 categories, then showed no significant increases or decreases in mean concentrations after the age of 30 years (Fig 3B). The interaction of age and cycle phase was not significant at a group level (Table 3), indicating that the relationship between cortisol and cycle phase did not change with age, but rather basal concentrations increased in both the follicular and luteal phases over time (Fig 3C). However, in female F1OZ (Fig 3D) who 
transitioned from pre-puberty to cycling during the study, there was a significant interaction between age and cycle phase, with a switch in the relationship between cortisol and the cycle phases (i.e., a crossover interaction) approximately 2 years after her first estrous cycle. Mean cortisol concentrations were initially lower in the follicular phase. Cortisol increased in both phases but the rate of increase was higher in the follicular phase.

At an individual level, age was a significant predictor of mean cortisol concentrations in all cycling females except F2OZ, with cortisol increasing with age in five females and decreasing with age in the oldest female (Table 3). In the two oldest females, F4OZ showed an overall increase with age, but no increase across age categories until age 5160 years; whereas F7NZ showed an overall decrease with age through the 51-60 age category, and then an increase in the last 4 years (age 67-71) after she was contracepted and acyclic. Female F5NZ showed no increase with age until the last 4 years (age 39-43) when mean urinary cortisol concentrations increased by $168 \%$. For the females that exhibited an increase in the last years of the study, it is possible the increased adrenal GC activity was confounded by declines in health, which for F2OZ resulted in euthanasia. 
Table 3: Age as a predictor of adrenal GC activity.

Individual, age range of analysis, age variable (age, age category, interaction of age and cycle), effect size with standard error (SE), Wald statistic, and p-value from GLMMs; and relative effect of age if significant. Age effect for individuals was modelled only during periods of normal cycling. Females listed in order of age during analysis. Degrees of freedom (df) was 1 in all pair-wise comparisons.

\begin{tabular}{|c|c|c|c|c|c|c|}
\hline Individual & $\begin{array}{l}\text { Age Range } \\
\text { of Analysis }\end{array}$ & Age Variable & $\begin{array}{l}\text { Effect Size } \\
\text { (SE) }\end{array}$ & Wald & $\mathbf{P}$ & Age Effect \\
\hline \multirow{8}{*}{$\begin{array}{l}\text { All elephants: } \\
\text { Across all } \\
\text { reproductive } \\
\text { states }\end{array}$} & \multirow[t]{8}{*}{$4-71$} & Age (years) & $0.001(0.001)$ & 1.225 & 0.289 & -- \\
\hline & & 0-10 (reference) & - & - & - & \\
\hline & & $11-20$ & $0.233(0.029)$ & 66.355 & $<0.001$ & Higher \\
\hline & & $21-30$ & $0.256(0.034)$ & 57.729 & $<0.001$ & Higher \\
\hline & & $31-40$ & $0.240(0.035)$ & 46.526 & $<0.001$ & Higher \\
\hline & & $41-50$ & $0.323(0.054)$ & 36.130 & $<0.001$ & Higher \\
\hline & & $51-60$ & $0.317(0.057)$ & 30.739 & $<0.001$ & Higher \\
\hline & & $61-71$ & $0.266(0.059)$ & 20.506 & $<0.001$ & Higher \\
\hline \multirow{8}{*}{$\begin{array}{l}\text { All elephants: } \\
\text { During normal } \\
\text { cycling only }\end{array}$} & \multirow[t]{8}{*}{$6-59$} & Age (years) & $0.005(0.001)$ & 14.590 & $<0.001$ & Increasing \\
\hline & & 0-10 (reference) & - & - & - & \\
\hline & & $11-20$ & $0.235(0.041)$ & 32.549 & $<0.001$ & Higher \\
\hline & & $21-30$ & $0.312(0.046)$ & 45.937 & $<0.001$ & Higher \\
\hline & & $31-40$ & $0.319(0.048)$ & 44.704 & $<0.001$ & Higher \\
\hline & & $41-50$ & $0.344(0.072)$ & 22.786 & $<0.001$ & Higher \\
\hline & & $51-60$ & $0.340(0.075)$ & 20.780 & $<0.001$ & Higher \\
\hline & & $\begin{array}{l}\text { Interaction of } \\
\text { Age and Cycle }\end{array}$ & $0.000(0.001)$ & 0.160 & 0.689 & -- \\
\hline \multirow[t]{4}{*}{ F1OZ } & \multirow[t]{4}{*}{$6-15$} & Age (years) & $0.044(0.007)$ & 39.33 & $<0.001$ & Increasing \\
\hline & & $0-10$ (reference) & - & - & - & \\
\hline & & $11-20$ & $0.242(0.036)$ & 44.501 & $<0.001$ & Higher \\
\hline & & $\begin{array}{l}\text { Interaction of } \\
\text { Age and Cycle }\end{array}$ & $-0.039(0.014)$ & 7.254 & 0.007 & Significant \\
\hline \multirow[t]{2}{*}{$\mathrm{F} 2 \mathrm{OZ}$} & \multirow[t]{2}{*}{$\sim 12-15$} & Age (years) & $0.006(0.024)$ & 0.055 & 0.816 & -- \\
\hline & & $\begin{array}{l}\text { Interaction of } \\
\text { Age and Cycle }\end{array}$ & $0.027(0.049)$ & 0.321 & 0.571 & -- \\
\hline \multirow[t]{4}{*}{$\mathrm{F} 3 \mathrm{OZ}$} & \multirow[t]{4}{*}{$11-26$} & Age (years) & $0.019(0.004)$ & 20.734 & $<0.001$ & Increasing \\
\hline & & $\begin{array}{l}11-20 \\
\text { (reference) }\end{array}$ & - & - & - & \\
\hline & & $21-30$ & $0.154(0.034)$ & 20.572 & $<0.001$ & Higher \\
\hline & & $\begin{array}{l}\text { Interaction of } \\
\text { Age and Cycle }\end{array}$ & $0.005(0.008)$ & 0.418 & 0.518 & -- \\
\hline
\end{tabular}




\begin{tabular}{|c|c|c|c|c|c|c|}
\hline Individual & $\begin{array}{l}\text { Age Range } \\
\text { of Analysis }\end{array}$ & Age Variable & $\begin{array}{l}\text { Effect Size } \\
\text { (SE) }\end{array}$ & Wald & $\mathbf{P}$ & Age Effect \\
\hline \multirow[t]{5}{*}{$\mathrm{F} 4 \mathrm{OZ}$} & \multirow[t]{5}{*}{$\sim 39-51$} & Age (years) & $0.017(0.004)$ & 15.120 & $<0.001$ & Increasing \\
\hline & & $\begin{array}{l}31-40 \\
\text { (reference) }\end{array}$ & - & - & - & \\
\hline & & $41-50^{*}$ & $0.047(0.074)$ & 0.406 & 0.524 & -- \\
\hline & & $51-60^{*}$ & $0.219(0.093)$ & 5.842 & 0.019 & Higher \\
\hline & & $\begin{array}{l}\text { Interaction of } \\
\text { Age and Cycle }\end{array}$ & $0.012(0.009)$ & 1.647 & 0.199 & -- \\
\hline \multirow[t]{5}{*}{ F5NZ (serum) } & \multirow[t]{5}{*}{$\sim 15-37$} & Age (years) & $0.000(0.002)$ & 0.017 & 0.896 & -- \\
\hline & & $\begin{array}{l}11-20 \\
\text { (reference) }\end{array}$ & - & - & - & \\
\hline & & $21-30$ & $0.035(0.035)$ & 0.983 & 0.321 & -- \\
\hline & & $31-40$ & $0.017(0.037)$ & 0.213 & 0.644 & -- \\
\hline & & $\begin{array}{l}\text { Interaction of } \\
\text { Age and Cycle }\end{array}$ & $-0.002(0.004)$ & 0.221 & 0.638 & -- \\
\hline \multirow[t]{2}{*}{ F5NZ (urine) } & \multirow[t]{2}{*}{$\sim 39-43$} & Age (years) & $0.124(0.014)$ & 82.255 & $<0.001$ & Increasing \\
\hline & & $\begin{array}{l}\text { Interaction of } \\
\text { Age and Cycle }\end{array}$ & $-0.040(0.029)$ & 2.007 & 0.157 & -- \\
\hline \multirow[t]{4}{*}{ F6NZ } & \multirow[t]{4}{*}{$\sim 28-41$} & Age & $0.010(0.003)$ & 15.888 & $<0.001$ & Increasing \\
\hline & & $\begin{array}{l}21-30 \\
\text { (reference) }\end{array}$ & - & - & - & \\
\hline & & $31-40$ & $0.100(0.024)$ & 17.393 & $<0.001$ & Higher \\
\hline & & $\begin{array}{l}\text { Interaction of } \\
\text { Age and Cycle }\end{array}$ & $0.003(0.005)$ & 0.285 & 0.593 & -- \\
\hline \multirow[t]{4}{*}{ F7NZ (serum) } & \multirow[t]{4}{*}{$\sim 46-59$} & Age (years) & $-0.080(0.003)$ & 7.259 & 0.007 & Decreasing \\
\hline & & $\begin{array}{l}41-50 \\
\text { (reference) }\end{array}$ & - & - & - & \\
\hline & & $51-60$ & $-0.041(0.020)$ & 4.260 & 0.039 & Lower \\
\hline & & $\begin{array}{l}\text { Interaction of } \\
\text { Age and Cycle }\end{array}$ & $-0.008(0.006)$ & 1.760 & 0.185 & -- \\
\hline F7NZ (urine) ${ }^{* *}$ & $\sim 67-71$ & Age (years) & $0.103(0.011)$ & 95.443 & $<0.001$ & Increasing \\
\hline
\end{tabular}

-- No significant difference

* Significant difference between time categories

** Reproductive state is acyclic 


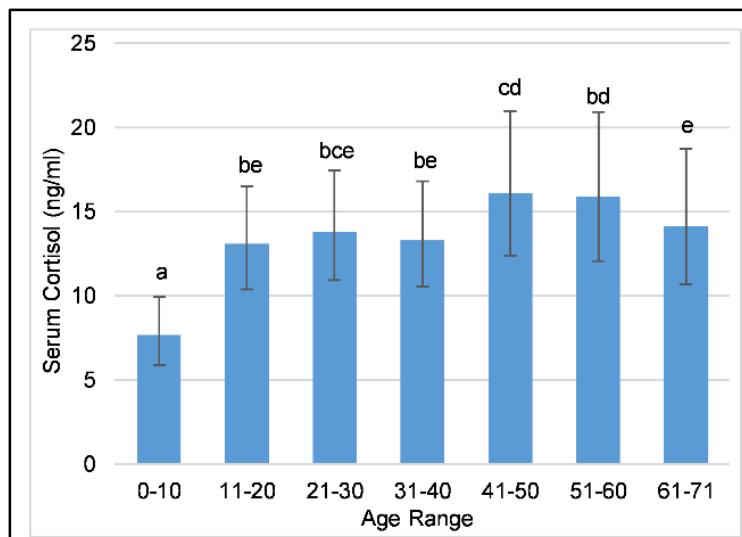

(A)

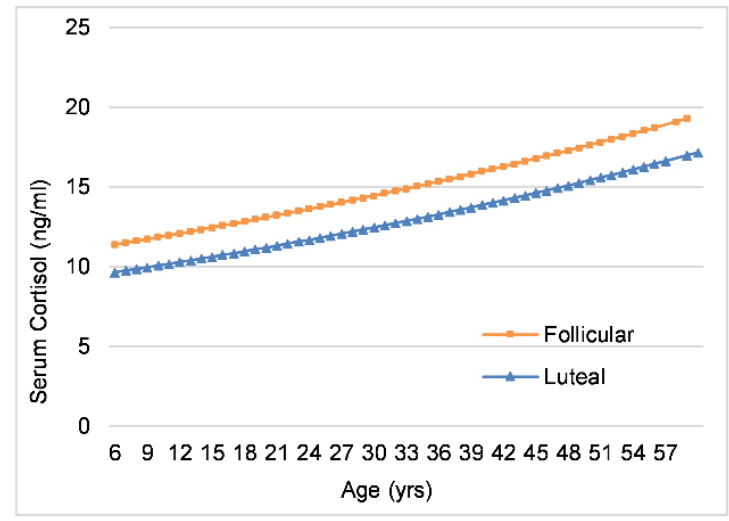

(C)

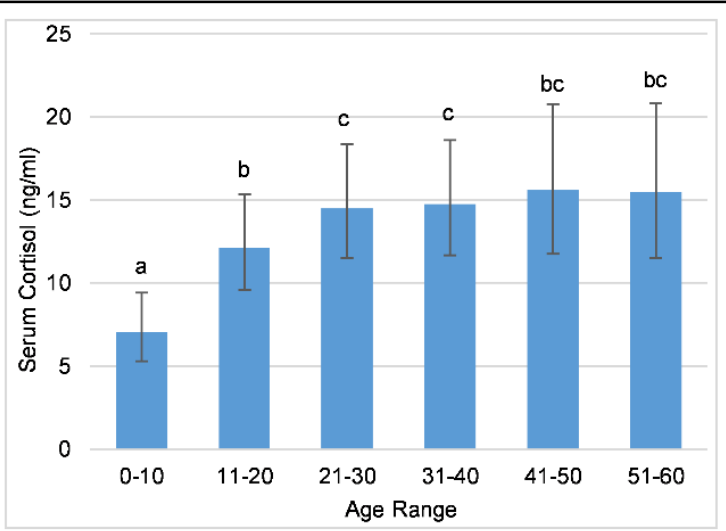

(B)

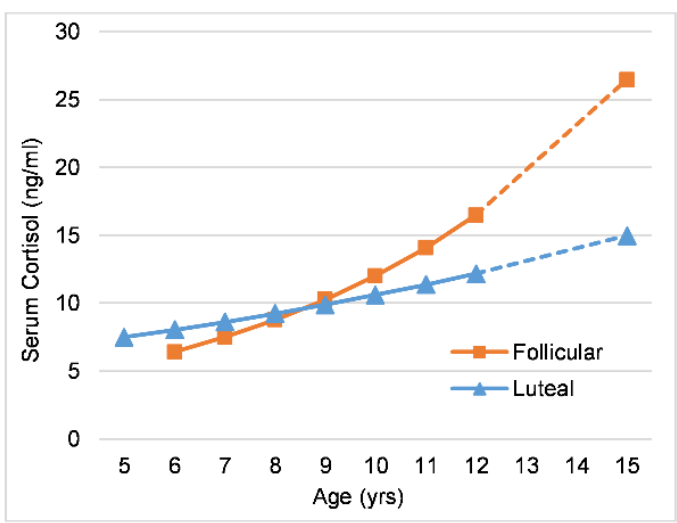

(D)

Fig 3: Age as a predictor of adrenal GC activity.

Predictions from GLMMs for serum cortisol concentration comparing mean values across age categories (error bars represent standard error of the prediction), and interactions of age and cycle phase. Letters denote significant differences in mean values. Dashed lines in interactions indicate a reproductive state other than normal cycling. (A) All elephants: Comparing mean values across age bins and including all reproductive states. (B) All elephants: Comparing mean values across age bins and including only periods of normal cycling. (C) All elephants: Interaction of age and cycle phase showing the relationship of cortisol and cycle phase with age. (D) F1OZ: Interaction of age and cycle phase showing the relationship of cortisol and cycle phase with age.

\section{Life events and adrenal GC activity}

A total of 22 life events (births, deaths, transfers) occurred during the study, representing

51 events experienced by nine elephants collectively. For 40 of the 51 events, there was

sufficient hormone data to analyze the adrenal GC response, taking into account

covariates of reproductive state and cycle phase. Of the 40 events, 38 were related to 
social life (births to a herdmate, deaths of offspring or a herdmate, and facility transfers) and 2 were related to a health decline preceding euthanasia. A social life event was a significant predictor of mean cortisol concentration in 15 of 38 events (39\%) for 8 of 9 females. Females with over 10 years of cortisol data $(n=6)$ experienced three to seven social life events, and the percentage of significant responses ranged from 0 to $50 \%$. Overall, this group of elephants did not show chronically elevated or suppressed concentrations of GCs during the study, and significant changes in mean cortisol concentrations returned to pre-event concentrations within 45 days in all but four cases ( 3 who experienced deaths of herdmates, 1 transfer of self).

Tables 4-8 show the life events that were modelled for each individual, comparing mean cortisol concentrations pre- and post-event. Post-events in 15-day periods are shown only if a significant difference was detected in any single time period compared to pre-event concentrations.

In cases where mean cortisol concentrations decreased in response to an event, pre-event concentrations for the individual were compared to their means across all reproductive states and during normal cycling (Appendix A: S1 Tables A-D) while accounting for increases in cortisol with age. This increases the likelihood that the observed decrease following an event did not represent a return to basal concentrations, but rather a response to the event itself. 


\section{Births to herdmates}

Of the three births that occurred, two had sufficient data to model. Birth to a herdmate was not a significant predictor of mean cortisol concentration (Table 4).

Table 4: Birth to a herdmate as a predictor of adrenal GC activity.

Fixed effects, effect size with standard error (SE), Wald statistic, and p-value from GLMMs, and whether mean cortisol concentration post-event was higher or lower than pre-event. Degrees of freedom (df) was 1 in all pair-wise comparisons.

\begin{tabular}{|l|l|l|l|l|c|}
\hline \multicolumn{1}{|c|}{ Event } & $\begin{array}{c}\text { Individual } \\
\text { Exposed to } \\
\text { Event }\end{array}$ & Effect Size (SE) & Wald & P & $\begin{array}{c}\text { Mean Post- } \\
\text { event Relative } \\
\text { to Pre-event }\end{array}$ \\
\hline $\begin{array}{l}\text { Birth1: Female calf (nzF1) } \\
\text { to F5NZ }\end{array}$ & F6NZ & $-0.229(0.193)$ & 1.396 & 0.237 & -- \\
\hline $\begin{array}{l}\text { Birth2: Male calf (nzM1) } \\
\text { to F5NZ }\end{array}$ & F6NZ & $0.020(0.109)$ & 0.033 & 0.856 & -- \\
\cline { 2 - 6 } & F7NZ & $-0.190(0.306)$ & 0.386 & 0.534 & -- \\
\hline
\end{tabular}

-- No significant difference

\section{Deaths of herdmates}

Six deaths occurred during the study, four at OZ (3 adult females, 1 adult male) and two at NZP (1 female calf, 1 adult female), representing 15 death events experienced by seven elephants collectively; all were modelled. Death of a herdmate was a significant predictor of mean cortisol concentration in $7(47 \%)$ of the events modelled (Table 5). The response to the same death varied among individuals (in 4 of 6 deaths), and individuals exhibited different responses to the deaths of different herdmates (in 4 of 5 individuals that experienced multiple deaths) (Table 5). 
Table 5: Death to a herdmate as a predictor of adrenal GC activity.

Fixed effects, effect size with standard error (SE), Wald statistic, and p-value from GLMMs, and whether the mean cortisol concentration post-event is higher or lower than pre-event. Degrees of freedom (df) was 1 in all pair-wise comparisons.

\begin{tabular}{|c|c|c|c|c|c|c|}
\hline Event & $\begin{array}{c}\text { Individual } \\
\text { Exposed } \\
\text { to Event }\end{array}$ & $\begin{array}{l}\text { Comparison } \\
\text { category }\end{array}$ & $\begin{array}{l}\text { Effect Size } \\
\quad \text { (SE) }\end{array}$ & Wald & $\mathbf{P}$ & $\begin{array}{l}\text { Mean Post- } \\
\text { event } \\
\text { Relative to } \\
\text { Pre-event }\end{array}$ \\
\hline \multirow{9}{*}{$\begin{array}{l}\text { Death 1: Female } \\
\text { (F4OZ) }\end{array}$} & F1OZ & Post & $0.090(0.100)$ & 0.809 & 0.368 & -- \\
\hline & \multirow[t]{4}{*}{$\mathrm{F} 2 \mathrm{OZ}$} & Post & $0.361(0.116)$ & 9.727 & 0.002 & Higher \\
\hline & & Post-day1-15 & $0.352(0.137)$ & 6.630 & 0.010 & Higher \\
\hline & & Post-day 16-30 & $0.369(0.137)$ & 7.289 & 0.007 & Higher \\
\hline & & Post-day31-45 & $0.351(0.179)$ & 3.851 & 0.050 & Higher \\
\hline & \multirow[t]{4}{*}{$\mathrm{F} 3 \mathrm{OZ}$} & Post & $0.139(0.065)$ & 4.615 & 0.032 & Higher \\
\hline & & Post-day1-15 & $0.139(0.059)$ & 5.539 & 0.019 & Higher \\
\hline & & Post-day 16-30 & $0.093(0.072)$ & 1.639 & 0.200 & -- \\
\hline & & Post-day31-45 & $0.000(0.000)$ & 0.000 & 1.000 & -- \\
\hline \multirow{5}{*}{$\begin{array}{l}\text { Death 2: Female } \\
\text { (ozF1) }\end{array}$} & \multirow[t]{4}{*}{$\mathrm{F} 3 \mathrm{OZ}$} & Post & $-0.037(0.090)$ & 0.167 & 0.683 & -- \\
\hline & & Post-day1-15 & $-0.153(0.039)$ & 15.816 & $<0.001$ & Lower \\
\hline & & Post-day 16-30 & $0.080(0.039)$ & 4.315 & 0.038 & Higher \\
\hline & & Post-day31-45 & $0.080(0.039)$ & 4.315 & 0.038 & Higher \\
\hline & $\mathrm{F} 4 \mathrm{OZ}$ & Post & $-0.246(0.261)$ & 0.888 & 0.346 & -- \\
\hline \multirow{2}{*}{$\begin{array}{l}\text { Death 3: Female } \\
\text { (ozF2) }\end{array}$} & $\mathrm{F} 3 \mathrm{OZ}$ & Post & $0.265(0.356)$ & 0.554 & 0.457 & -- \\
\hline & $\mathrm{F} 4 \mathrm{OZ}$ & Post & $-0.273(0.207)$ & 1.752 & 0.186 & -- \\
\hline \multirow{6}{*}{$\begin{array}{l}\text { Death 4: Male } \\
\text { (ozM1) }\end{array}$} & F1OZ & Post & $-0.346(0.119)$ & 8.468 & 0.004 & Lower* \\
\hline & $\mathrm{F} 3 \mathrm{OZ}$ & Post & $0.316(0.238)$ & 1.756 & 0.185 & -- \\
\hline & \multirow[t]{4}{*}{$\mathrm{F} 4 \mathrm{OZ}$} & Post & $0.315(0.141)$ & 4.969 & 0.026 & Higher \\
\hline & & Post-day1-15 & no data & no data & no data & -- \\
\hline & & Post-day $16-30$ & $0.315(0.109)$ & 8.353 & 0.004 & Higher \\
\hline & & Post-day31-45 & $-0.210(0.099)$ & 4.438 & 0.035 & Lower \\
\hline \multirow{7}{*}{$\begin{array}{l}\text { Death 5: Female } \\
\text { calf (nzF1) }\end{array}$} & \multirow[t]{4}{*}{ F5NZ } & Post & $-0.145(0.164)$ & 0.785 & 0.376 & Lower \\
\hline & & Post-day1-15 & $-0.317(0.117)$ & 7.293 & 0.007 & Lower \\
\hline & & Post-day $16-30$ & $0.197(0.151)$ & 1.701 & 0.192 & -- \\
\hline & & Post-day31-45 & $0.000(0.000)$ & 0.0 & 1.0 & -- \\
\hline & \multirow[t]{3}{*}{ F6NZ } & Post & $-0.134(0.144)$ & 0.862 & 0.353 & -- \\
\hline & & Post-day 1-15 & $-0.197(0.065)$ & 9.205 & 0.002 & Lower \\
\hline & & Post-day 16-30 & $0.300(0.051)$ & 34.097 & $<0.001$ & Higher \\
\hline
\end{tabular}




\begin{tabular}{|l|l|l|l|l|l|c|}
\hline \multicolumn{1}{|c|}{ Event } & $\begin{array}{c}\text { Individual } \\
\text { Exposed } \\
\text { to Event }\end{array}$ & $\begin{array}{c}\text { Comparison } \\
\text { category }\end{array}$ & $\begin{array}{c}\text { Effect Size } \\
\text { (SE) }\end{array}$ & Wald & P & $\begin{array}{c}\text { Mean Post- } \\
\text { event } \\
\text { Relative to } \\
\text { Pre-event }\end{array}$ \\
\hline & & Post-day31-45 & $0.097(0.051)$ & 3.556 & 0.059 & -- \\
\cline { 2 - 7 } & F7NZ & Post & $-0.060(0.075)$ & 0.636 & 0.425 & -- \\
\hline $\begin{array}{l}\text { Death 6: Female } \\
\text { (F6NZ) }\end{array}$ & F5NZ & Post & $0.046(0.114)$ & 0.163 & 0.686 & -- \\
\cline { 2 - 7 } & F7NZ & Post & $0.041(0.110)$ & 0.139 & 0.709 & -- \\
\hline
\end{tabular}

-- No significant difference

* 60-day model (60 days pre- and post-event)

The death of F4OZ had a significant effect on two of three herdmates, with higher mean cortisol concentrations in the time periods following her death (Table 5). Female F2OZ exhibited increased cortisol for at least 45 days (Fig 4A); whereas F3OZ showed an increase only in the first 15 days (Fig 4B). The death of female ozF1 had a significant effect on one of two herdmates. Female F3OZ exhibited decreased cortisol in the first 15 days, then concentrations increased compared to pre-event concentrations for at least the next 30 days (Fig 4C). The deaths of females ozF2 and F6NZ had no significant effect on mean cortisol concentration of herdmates.

The death of female calf nzF1 (at age 1.4 years) had a significant effect on two of three females. In both individuals, mean cortisol was lower following her death (Table 4). The mother of the calf, F5NZ, showed a decrease in cortisol in the first 15 days, then returned to pre-event concentrations (Fig 4E), whereas F6NZ showed a decrease in cortisol for the first 15 days then an increase and a return to pre-event concentrations within 45 days (Fig 4F). 
The death of male ozM1 had a significant effect on two of three females. F4OZ exhibited higher mean cortisol concentrations in the 30-day time period following his death (Table 5) but showed increased concentrations only in days 16-30 following his death (Fig 4D). F1OZ exhibited decreased cortisol in the 60-day time period following his death and a $71 \%$ decrease in the 30 -day time period. 


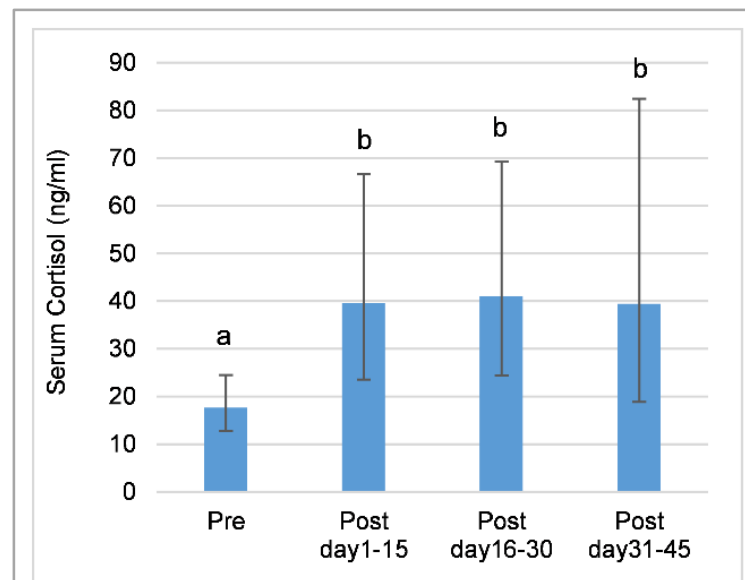

(A)

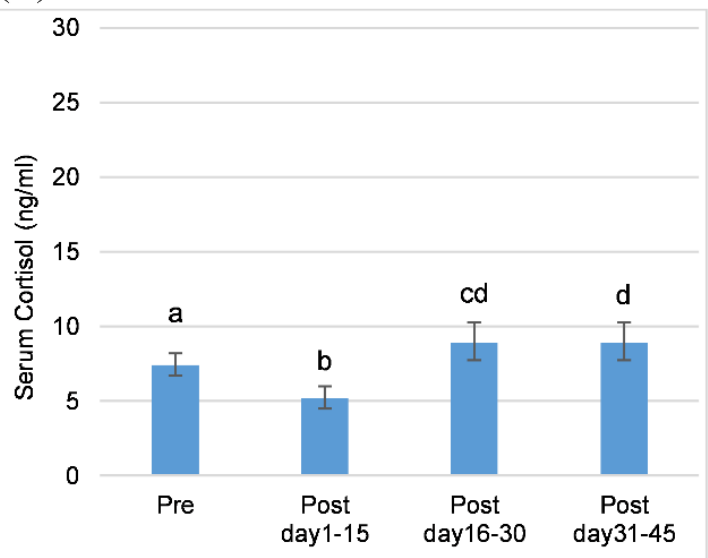

(C)

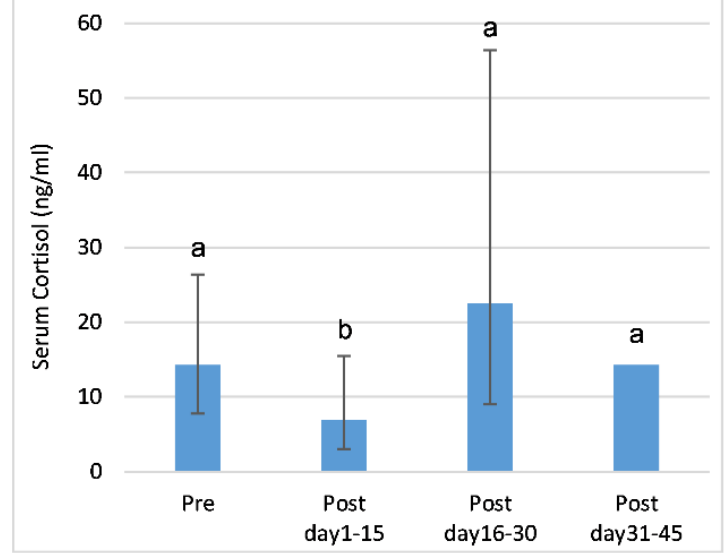

(E)

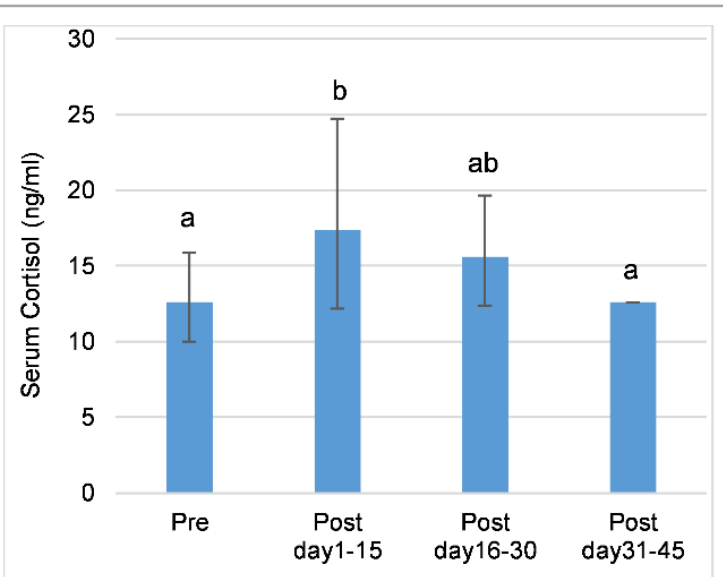

(B)

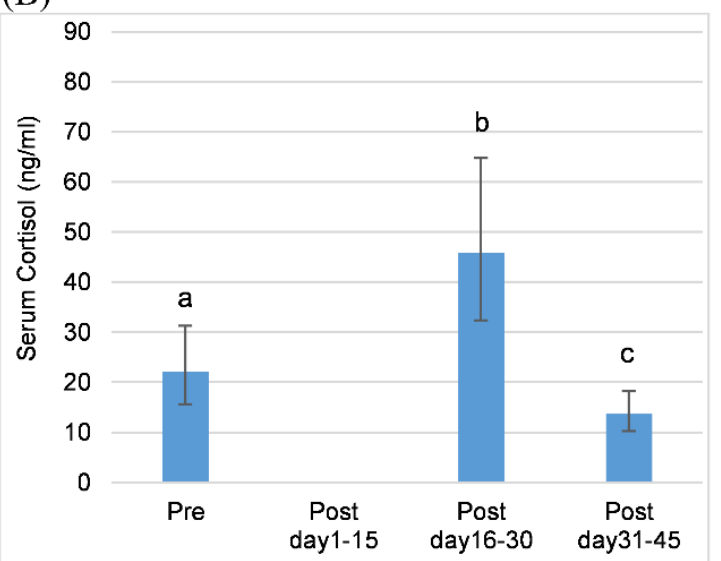

(D)

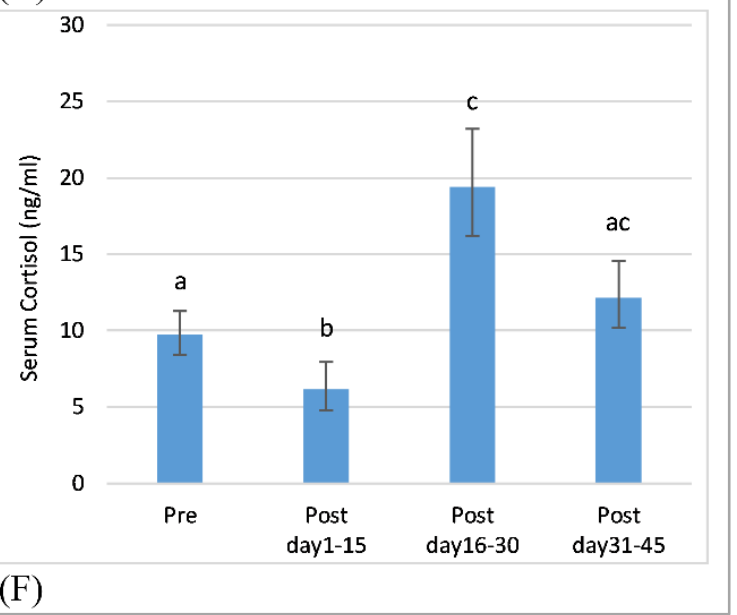

Fig 4: Death of a herdmate as a predictor of adrenal GC activity.

Predictions from GLMMs for cortisol concentration comparing mean values in the 30 days prior to death and the 45 days post-death in 15-day time blocks (error bars represent standard error of the prediction). Letters denote significant differences in mean values across time periods. (A) Death 1, F2OZ response: Post $>$ Pre in 15-day time periods post death; values remained above pre-event concentrations for at least 45 days. (B) Death 1, F3OZ response: Post > Pre in first 15 days post death; values returned to pre-event concentrations after 15 days. (C) Death 2, F3OZ response: values decreased then increased to above preevent concentrations for at least 30 days. (D) Death 4, F4OZ response: values increased then decreased to 
below pre-event concentrations. (E) Death 5, F5NZ response: Post < Pre in first 15-days post death; values returned to pre-event concentrations after 15 days. (F) Death 5, F6NZ response: values decreased then increased above pre-event concentrations then returned to pre-event concentrations after 30 days.

\section{Transfers of herdmates}

Ten individuals were transferred during the study: 3 at OZ (2 transfers in, 1 transfer out) and 7 at NZP (6 transfers in, 1 transfer out), with 7 transfers of single elephants and 1 group transfer. Of these 8 transfer events, 6 were modelled for transfer of herdmate, representing 17 herdmate transfer events experienced by 8 elephants collectively. The transfer in of F5NZ could not be modelled for herdmates because there was not sufficient data. The transfer in of an adult male to NZP in the last year of the study was not modelled because only 2 of 4 females had sufficient cortisol data, and both of those had confounding health declines. Transfer of a herdmate was a significant predictor of mean cortisol concentration in 5 (29\%) of the 17 events (Table 6), all of which were in response to transfers of two males, although responses differed. 
Table 6: Transfer of a herdmate as a predictor of adrenal GC activity.

Fixed effects, effect size with standard error (SE), Wald statistic, and p-value from GLMMs, and whether the mean cortisol concentration post-event was higher or lower than pre-event. Degrees of freedom (df) was 1 in all pair-wise comparisons.

\begin{tabular}{|c|c|c|c|c|c|c|}
\hline Event & $\begin{array}{c}\text { Individual } \\
\text { Exposed to } \\
\text { Event }\end{array}$ & $\begin{array}{l}\text { Comparison } \\
\text { category }\end{array}$ & $\begin{array}{l}\text { Effect Size } \\
\quad \text { (SE) }\end{array}$ & Wald & $\mathbf{P}$ & $\begin{array}{c}\text { Mean } \\
\text { Post-event } \\
\text { Relative to } \\
\text { Pre-event }\end{array}$ \\
\hline \multirow{3}{*}{$\begin{array}{l}\text { Transfer 1: In of } \\
\text { female (F2OZ) }\end{array}$} & F1OZ & Post & $0.006(0.132)$ & 0.002 & 0.964 & -- \\
\hline & $\mathrm{F} 3 \mathrm{OZ}$ & Post & $-0.115(0.116)$ & 0.988 & 0.320 & -- \\
\hline & $\mathrm{F} 4 \mathrm{OZ}$ & Post & $-0.045(0.136)$ & 0.109 & 0.741 & -- \\
\hline \multirow{4}{*}{$\begin{array}{l}\text { Transfer 2: In of } \\
\text { male (ozM2) }\end{array}$} & F1OZ & Post & $-0.049(0.097)$ & 0.257 & 0.612 & -- \\
\hline & $\mathrm{F} 2 \mathrm{OZ}$ & Post & $0.207(0.093)$ & 5.011 & 0.025 & Higher \\
\hline & $\mathrm{F} 3 \mathrm{OZ}$ & Post & $-0.192(0.096)$ & 4.024 & 0.045 & Lower \\
\hline & $\mathrm{F} 4 \mathrm{OZ}$ & Post & $-0.208(0.070)$ & 8.928 & 0.003 & Lower \\
\hline \multirow{2}{*}{$\begin{array}{l}\text { Transfer 3: Out } \\
\text { of female (ozF3) }\end{array}$} & $\mathrm{F} 3 \mathrm{OZ}$ & Post & $-0.062(0.137)$ & 0.204 & 0.652 & -- \\
\hline & $\mathrm{F} 4 \mathrm{OZ}$ & Post & $0.029(0.202)$ & 0.201 & 0.654 & -- \\
\hline \multirow{2}{*}{$\begin{array}{l}\text { Transfer 4: In of } \\
\text { female (F10NZ) }\end{array}$} & F5NZ & Post & $0.022(0.108)$ & 0.043 & 0.325 & -- \\
\hline & F7NZ & Post & $-0.079(0.144)$ & 0.487 & 0.485 & -- \\
\hline \multirow{2}{*}{$\begin{array}{l}\text { Transfer 5: In of } \\
\text { female group } \\
\text { (F9NZ, F8NZ, } \\
\text { F11NZ) }\end{array}$} & F5NZ & Post & $0.161(0.144)$ & 1.247 & 0.264 & -- \\
\hline & F7NZ & Post & $0.086(0.098)$ & 0.768 & 0.380 & -- \\
\hline \multirow{8}{*}{$\begin{array}{l}\text { Transfer 6: Out } \\
\text { of male (nzM1) }\end{array}$} & \multirow[t]{2}{*}{ F5NZ } & Post & $0.260(0.112)$ & 5.387 & 0.020 & Higher \\
\hline & & Post-day $1-15^{*}$ & $0.260(0.104)$ & 6.266 & 0.012 & Higher \\
\hline & F7NZ & Post & $-0.136(0.168)$ & 0.651 & 0.420 & -- \\
\hline & F8NZ & Post & $0.212(0.218)$ & 0.943 & 0.331 & -- \\
\hline & \multirow[t]{4}{*}{ F9NZ } & Post & $0.759(0.189)$ & 16.165 & $<0.001$ & Higher \\
\hline & & Post-day 1-15 & $0.612(0.166)$ & 13.575 & $<0.001$ & Higher \\
\hline & & Post-day 16-30 & $0.980(0.182)$ & 29.038 & $<0.001$ & Higher \\
\hline & & Post-day31-45 & $0.269(0.152)$ & 3.125 & 0.077 & -- \\
\hline
\end{tabular}

-- No significant difference

* GLMM showed no difference between Days 16-45 and pre-event

The transfer in of an adult male (ozM2) had a significant effect on 3 of 4 females. F2OZ showed increased mean cortisol concentrations in the 120-day time period after his transfer; whereas F3OZ and F4OZ showed decreased concentrations (Table 6). The 
transfers in of a juvenile female (F2OZ), adult female (F10NZ), and a group of adult females had no significant effects on cortisol concentrations in herdmates (Table 6).

The transfer out of a sub-adult male (nzM1, at age 14) had a significant effect on 2 of 4 females. F5NZ (mother) and F9NZ both showed increases in mean cortisol concentration in the 30-day time period following his transfer (Table 6). F5NZ returned to pre-event concentrations after 15 days; F9NZ returned to pre-event concentrations after 30 days (Fig 5A). The transfer out of an adult female (ozF3) had no significant effect on cortisol concentrations in OZ herdmates (Table 6)

\section{Transfers of self}

Six females in the study were transferred, three of which were modelled. One female did not have sufficient cortisol data to model the transfer, and two females were not modelled because they did not exhibit a period of normal cycling during the study. Transfer of self was a significant predictor of mean cortisol concentration in all three modelled individuals, although responses differed (Table 7). 
Table 7: Transfer of self as a predictor of adrenal GC activity.

Fixed effects, effect size with standard error (SE), Wald statistic, and p-value from GLMMs, and whether the mean cortisol concentration post-event was higher or lower than pre-event. Degrees of freedom (df) was 1 in all pair-wise comparisons.

\begin{tabular}{|c|c|c|c|c|c|}
\hline Event & $\begin{array}{c}\text { Comparison } \\
\text { category }\end{array}$ & Effect Size (SE) & Wald & $\mathbf{P}$ & $\begin{array}{c}\text { Mean Post- } \\
\text { event Relative } \\
\text { to Pre-event }\end{array}$ \\
\hline \multirow{4}{*}{$\begin{array}{l}\text { Transfer of self } 1 \text { : } \\
\text { F5NZ }\end{array}$} & Post & $0.257(0.157)$ & 2.693 & 0.101 & -- \\
\hline & Post-day1-15 & $0.373(0.164)$ & 5.146 & $\mathbf{0 . 0 2 3}$ & Higher \\
\hline & Post-day 16-30 & $0.180(0.147)$ & 1.492 & 0.222 & -- \\
\hline & Post-day31-45 & $-0.044(0.164)$ & 0.070 & 0.791 & -- \\
\hline \multirow{4}{*}{$\begin{array}{l}\text { Transfer of self 2: } \\
\text { F8NZ }\end{array}$} & Post & $0.248(0.073$ & 11.349 & $<0.001$ & Higher \\
\hline & Post-day1-15 & $0.310(0.071)$ & 19.067 & $<0.001$ & Higher \\
\hline & Post-day16-30 & $0.185(0.071)$ & 6.791 & 0.009 & Higher \\
\hline & Post-day31-45 & $0.100(0.071)$ & 1.984 & 0.159 & -- \\
\hline \multirow{4}{*}{$\begin{array}{l}\text { Transfer of self 3: } \\
\text { F9NZ }\end{array}$} & Post & $-0.273(0.110)$ & 6.203 & 0.013 & Lower \\
\hline & Post-day1-15 & $-0.153(0.099)$ & 2.402 & 0.121 & -- \\
\hline & Post-day 16-30 & $-0.393(0.099)$ & 15.805 & $<0.001$ & Lower \\
\hline & Post-day31-45 & $-0.157(0.070)$ & 5.015 & 0.025 & Lower \\
\hline
\end{tabular}

-- No significant difference

Females F5NZ and F8NZ showed higher mean cortisol concentrations post-transfer; whereas F9NZ showed lower concentrations (Table 7). F5NZ exhibited increased cortisol only in the first 15 days before returning to pre-transfer concentrations (Fig 5B), while F8NZ exhibited increased cortisol for 30-days (Fig 5C). By contrast, cortisol was decreased in F9NZ through at least 45 days (Fig 5D). 


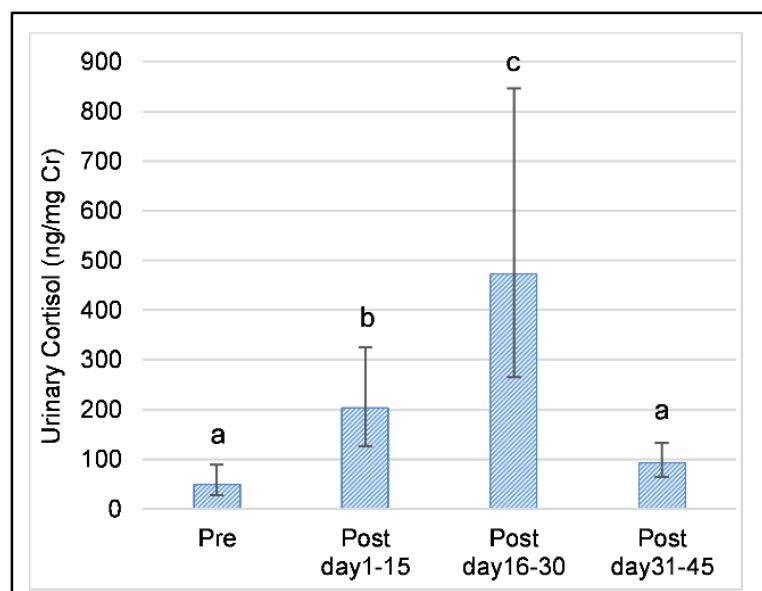

(A)

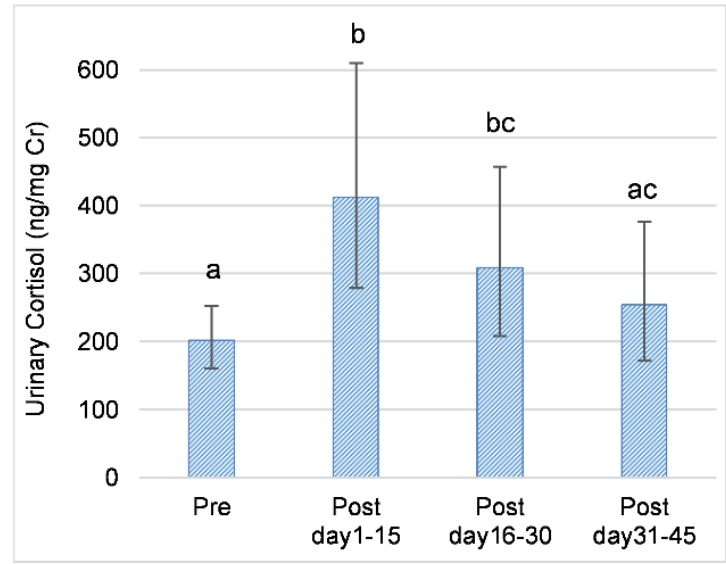

(C)

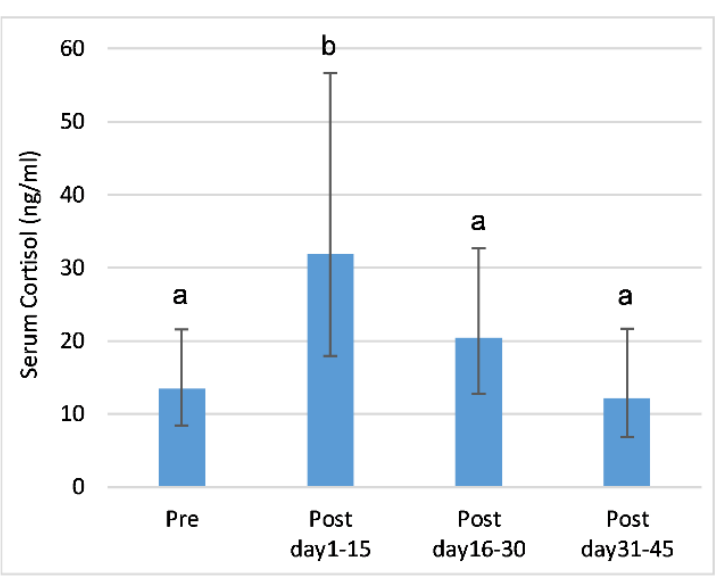

(B)

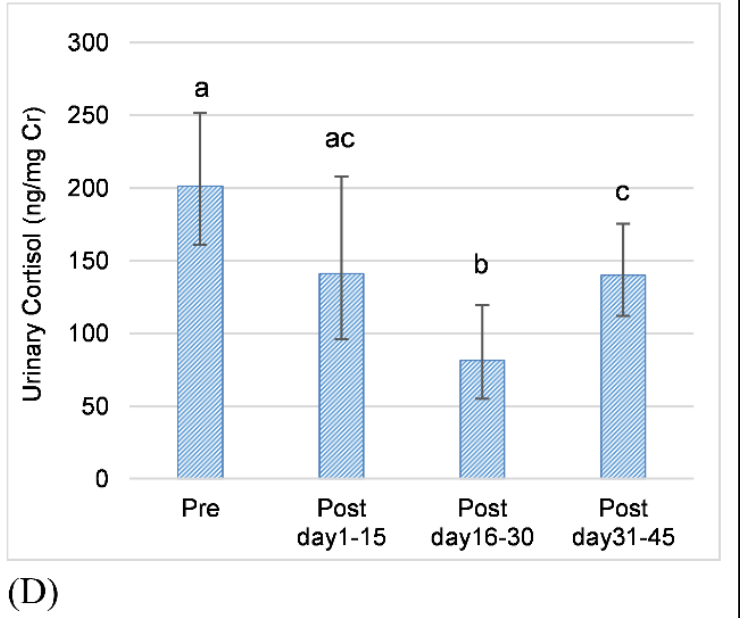

Fig 5: Facility transfer as a predictor of adrenal GC activity.

Predictions from GLMMs for cortisol concentration comparing mean values in the 30 days prior to transfer and the 45 days post-transfer in 15-day time blocks (error bars represent standard error of the prediction). Letters denote significant differences in mean values across time periods. (A) Transfer 6 of herdmate, F9NZ response: Post > Pre in the first 15 days and increased again in the next 15 days; values returned to pre-event concentrations after 30 days. (B) F5NZ transfer of self: Post > Pre in the first 15 days after the transfer; values returned to pre-event concentrations after 15 days. (C) F8NZ transfer of self: Post > Pre in the first 30 days after transfer; values returned to pre-event concentrations after 30 days. (D) F9NZ transfer of self: No significant difference in the first 15 days, then Post < Pre for at least the next 30 days.

\section{Death of self}

Four females were humanely euthanized during the study; two had sufficient cortisol data

to be modelled. The period of health decline leading to euthanasia was a significant

predictor of mean cortisol concentration in one female (F6NZ) but not the other (F4OZ) 
(Table 8). F6NZ showed significantly higher mean cortisol concentration in the final 30 days of life, with a sharp increase in the week prior to euthanasia.

Table 8: Death of self as a predictor of adrenal GC activity.

Fixed effects, effect size with standard error (SE), Wald statistic, and p-value from GLMMs, and whether the mean cortisol concentration in the final 30 days before death was higher or lower than the previous 30 days. Degrees of freedom (df) is 1 in all pair-wise comparisons.

\begin{tabular}{|l|c|r|r|c|}
\hline \multicolumn{1}{|c|}{ Event } & Effect Size (SE) & \multicolumn{1}{c|}{ Wald } & P & $\begin{array}{c}\text { Mean Final 30 } \\
\text { Days Relative to } \\
\text { Previous 30 Days }\end{array}$ \\
\hline Death of self 1: F4OZ & $-0.225(0.184)$ & 1.497 & 0.221 & -- \\
\hline Death of self 2: F6NZ & $0.355(0.088)$ & 16.132 & $<\mathbf{0 . 0 0 1}$ & Higher \\
\hline
\end{tabular}

-- No significant difference

\section{Discussion}

This study confirmed the presence of intrinsic cortisol patterns associated with reproductive state, ovarian cycle phase, and age in female Asian elephants, similar to that shown in other species and in previous studies of elephants [17, 37, 40, 41, 72-76]. Results further highlight the importance of accounting for these patterns when evaluating the impact of environmental change on adrenal GC activity. Concentrations of serum and urinary cortisol covaried more consistently with physiological changes (changes in reproductive state, ovarian cycle phase, and age) than with social changes (births, deaths, and transfers), with no differences observed between the two facilities. Interestingly, effects of transfers on cortisol were related to movement of males only, suggesting the influence of bulls in a herd should be explored further. Differing responses to social changes reinforce the notion that welfare should be assessed on an individual basis, and 
should consider variability in GCs within individuals in addition to average concentrations.

\section{$\underline{\text { Reproduction and adrenal GC activity }}$}

Reproductive state (prepubertal, normal cycling, pregnant, lactational anestrus, irregular cycling, contracepted, acyclic) was a significant predictor of mean serum and urinary cortisol concentrations in $80 \%(4 / 5)$ of the females that changed reproductive states during the study, but the pattern varied among individuals.

\section{Puberty}

In the two females that reached puberty during the study, mean cortisol was lower in prepuberty compared to normal cycling for one female (zoo-born) and higher for the other (wild-born). Variability (CV) was lower in prepuberty for both females. The differences between these two females may be due in part to social factors. The zoo-born female was born into a herd and had the social support of adult female elephants from birth; whereas the prepubertal timeframe for the wild-born elephant coincided with assimilation into a herd for the first time since she was orphaned.

\section{Ovarian cycle}

Ovarian cycle phase was a significant predictor of mean serum and urinary cortisol concentrations in all elephants combined and in 44\% (4/9) of the individual females. In general, higher mean cortisol concentration were observed in the follicular compared to the luteal phase. Although as a group, both parous/multiparous and nulliparous females exhibited this pattern, the interaction of parity and cycle phase was statistically 
significant, with cycle phase having a larger effect on cortisol in parous/multiparous females compared to nulliparous females, and a greater percentage of parous females exhibited higher mean values during the follicular phase. These findings confirmed cyclic patterns of serum cortisol in parous Asian elephants reported by Fanson et al. [37], where concentrations increased in the first half of the follicular phase, peaked around ovulation, then declined quickly and remained low throughout the luteal phase. By contrast, in nulliparous females who failed to conceive despite repeated mating or artificial insemination attempts, the cortisol pattern across the ovarian cycle was not as clear [37], a finding similar to ours. Thus, changes in GCs across the estrous cycle may promote normal ovarian function, including ovulation and formation of the corpus luteum (reviewed in Tetsuka [77]). Suppressive or deleterious effects of elevated cortisol have been a main focus in studies of reproductive dysfunction in elephants $[68,78,79]$; the potential permissive or stimulatory effects of cortisol have yet to be fully explored in this species. Increase in GCs prior to or around ovulation have been found in well-studied model species [72] and in a growing number of wildlife and domesticated species (e.g., giant panda (Ailuropoda melanoleuca) [73], musk shrew (Suncus murinus) [75]; sheep [74]). In rats, the peak in GC concentration at proestrus is due to a change in the amplitude of the circadian rhythm rather than an overall upregulation of adrenal GC activity. Specifically, the morning peak in GCs is highest in proestrus and lowest immediately following ovulation, but nadir values remain the same $[72,80]$. These findings imply that short-term increases in GCs may have stimulatory effects on reproductive function, and thus the pattern of GC secretion may be more biologically relevant than mean values. Monitoring the circadian variation in GC concentrations 
surrounding reproductive states of both sexes may help elucidate the interaction between the hypothalamic-pituitary-gonadal (HPG) and HPA axes in elephants, and with longterm monitoring how these circadian patterns change with age and the implication of those changes. Variability (CV) in cortisol also differed across the ovarian cycle, and was significantly higher in the luteal compared to the follicular phase for all elephants combined, although only those females that showed a difference in mean cortisol between cycle phases also showed a difference in variability and the pattern varied across individuals.

\section{Pregnancy}

Full pregnancies were documented in two females; one exhibited a higher mean and CV in cortisol concentrations during pregnancy compared to cycling while the other showed no significant differences between pregnant and cycling states. In other mammalian species, GC concentrations are elevated during gestation and around parturition [4, 81]. Similar gestational increases in cortisol have been found in elephants, but not consistently, whereas increases around parturition have been widely reported. Gobush et al. [25] found that fecal GC metabolites (fGCMs) increased with stage of gestation in free-ranging African elephants, while in Asian elephants, overall cortisol concentrations did not appear to be significantly altered during gestation, but increased sharply peripartum $[40,41,82]$. In examination of daily serum samples at the end of gestation, Meyer et al. [82] observed additional surges in cortisol between 8 and 11 days prior to parturition, which was supported by Kaewmanee et al. [83] who reported cortisol increases before parturition, 5-10 days in African elephants and 30-40 days in Asian 
elephants. Together, these findings imply that cortisol plays a role in gestation and parturition in elephants as in other species. Edwards and Boonstra [81] found that in most taxa, maternal free GCs increase in late gestation by means of species-specific strategies involving increased total GC secretion, decreased corticosteroid-binding globulin, or maturation and activation of fetal adrenals, all of which lead to increased exposure of the fetus to GCs essential for development. The cortisol surges observed at the end of gestation in elephants could be part of an endocrine cascade that facilitates parturition similar to that described for other mammalian species, whereby parturition is triggered by activation of the fetal HPA axis and an increase in fetal cortisol secretion (reviewed in Meyer et al. [82]).

\section{Lactational anestrus}

In the two females that gave birth during the study, mean cortisol was highest during lactational anestrus compared to all other reproductive states, which provides evidence that milk production and/or rearing a calf is physiologically and/or physically taxing. From a metabolic perspective, pregnancy and lactation are metabolically demanding due to the increased requirement for nutrients such as glucose $[84,85]$. The primary role of GCs at basal concentrations is energy regulation [12], so with increased energy demands one would expect increased GC production during lactational anestrus. Parental care also can be associated with increased "stress", albeit generally positive in nature. Pokharel et al. [86] found that fecal GC metabolites among free-ranging adult female Asian elephants were positively correlated with the number of suckling calves and lactating females in a herd, attributing elevated CGs to the stressor of predation threats to suckling calves and 
the metabolic and nutritional demands of lactation. Interestingly, the one female who gave birth to two calves during the study had a lower mean cortisol concentration and CV during gestation and lactational anestrus with the second calf (a male) as compared to the first (a female), which may indicate that gestation and rearing gets easier with experience. Alternatively, sex of the calf may have influenced cortisol secretion and so warrants further investigation.

\section{Demographic factors and adrenal GC activity}

Age across females

Age was a significant predictor of serum and urinary mean cortisol concentrations in all elephants combined, with concentrations being lowest in the 0-10-year age category, higher in the age categories spanning 11-60 years with 41-60 years being highest, then lower in the oldest age category ( $>61$ years of age). The effect of age on cortisol concentrations was confounded with reproductive state, however, so both factors should be considered when examining extrinsic effects on adrenal GC activity. Although these variables were confounded, the effect of age across all reproductive states reflects what we might see in a group of elephants with an age structure that includes females undergoing puberty or senescence, adult females that are parous and nulliparous, and females that cycle normally.

In females combined during normal cycling, mean cortisol concentration increased overall with age, and also across age categories with the lowest concentrations in the 0 10-year age category, which then increased in the 11-20 and 21-30 categories, then 
remained fairly steady across the 21-60 age categories. Previous studies have shown that serum cortisol also increases with age across Asian elephant bulls [38, 39]. The difference in covariance with age across all reproductive states versus limited to normal cycling may in part be due to the exclusion of the acyclic older female, F7NZ, who was the only individual that showed a negative correlation between age and cortisol concentration; and exclusion of pregnancies and lactational anestrus at ages 11-20 years.

\section{Aging within individuals}

In individual females during normal cycling, mean cortisol increased with age in $71 \%$ $(5 / 7)$ of the females, decreased in the oldest female, and showed no significant change in the other. The association between cortisol and age is complex, as highlighted by dissimilar patterns in the oldest females. The female that cycled normally until she died at age 51 showed an overall increase in cortisol with age; whereas the female who cycled normally until she was contracepted at age 59 showed an overall decrease with age. She did show an increase in her last four years at ages 67-71 while was acyclic, so this increase is not related to any change in cyclicity but likely to other factors such as health issues with advancing age.

Individuals showed different patterns in the relationship between cortisol and cycle phase with age. With exception of one female, the interaction between age and cycle phase was not significant, indicating concentrations overall increased (or decreased) equally in both the follicular and luteal phases over time. However, visual inspection showed a trend towards higher rates of change in the follicular phase in the youngest females versus 
higher rates in the luteal phase in the oldest females. The youngest female initially showed higher cortisol concentrations during the luteal phase, but the higher rate of increase in the follicular phase resulted in a switch about 2 years after her first estrous cycle such that concentrations became higher during the follicular phase, similar to the other females. Given the role of GCs in follicular development/maturation and ovulation, it stands to reason that the relationship between cortisol and ovarian activity would become stronger in the early post-pubertal years. Further investigation into the relationship between cortisol and ovarian activity over time may help elucidate any role cortisol plays in follicular development, and how this may be associated with reproductive potential.

\section{Origin of birth}

Surprisingly, mean cortisol concentrations did not differ between wild-born (wild caught or orphans) and zoo-born females. Prado-Oviedo et al. [87] found that wild-born elephants imported to North American zoos were on average 20 years older than captiveborn elephants. Consistent with those demographics, origin was confounded with age in this study as wild-born females were in the higher age categories. The one exception was the wild-born Bornean elephant (age 9-15 years) who exhibited mean cortisol concentrations that were approximately double that of all other females, which may be attributed to challenges in her early life experience. In the wild, female elephants generally associate with their natal herd throughout their lives, so separation of mother and offspring represents a challenging life event. This female was found injured as an orphan, blinded in one eye by an apparent gunshot wound. She formed a social bond with 
her caretaker and had encounters with wild elephants, but it is unknown whether she had opportunities to form social bonds with other elephants before she was imported at $\sim 6$ years of age. Four of the other wild-born females in this study lived together in the same orphanage in Sri Lanka, so at least had the opportunity to interact with other elephants. In other species, cortisol secretion later in life can be influenced by early life trauma [88] or early social experience on development of the stress response [89, 90]. Estrous cycle characteristics for the Bornean female also were different; her estrous cycles were significantly longer in duration, and progesterone peaks were higher [55]. It is also plausible there are inherent genetic differences since it has been shown Bornean elephants are a genetically distinct population [91, 92], however there are no published hormone data on other Bornean females for comparison.

\section{$\underline{\text { Life events and adrenal GC activity }}$}

Social life events (birth to a herdmate, death of a herdmate, transfer in or out of a herdmate or self) were significant predictors of mean serum and urinary cortisol concentration in $89 \%(8 / 9)$ of the females and in $39 \%(15 / 38)$ of the events experienced by females collectively. In all but four instances, cortisol returned to pre-event concentrations within 45 days, indicating an adaptive physiological response or resilience, whereby GCs concentrations were temporarily elevated then subsequently returned to individual baseline concentrations. In general, the adrenal GC responses observed in this study were short-lived and there was no evidence of hyper- or hypocortisol production in any of these females. 


\section{Births to herdmates}

Birth to a herdmate was not a significant predictor of mean cortisol concentration in that neither of the females who experienced this event type showed any difference in cortisol post-birth. Although results did not provide evidence of a change in adrenal GC activity in response to the birth in the timeframe measured, birth within an elephant herd is generally considered a positive stimulus. Calves also have a beneficial impact on a herd, stimulating play behavior and affiliative interactions [93]. Wild elephant herds typically include calves and juveniles, and there is evidence that allomothering strengthens social bonds and improves a female's ability to successfully mother her own calves in the future $[22,94]$, and as such the addition of calves through successful breeding promotes normal behavior for this species in addition to giving females the opportunity to reproduce.

\section{Deaths of herdmates}

Death of a herdmate was a significant predictor of mean cortisol concentration in $-47 \%$ (7/15) of the death events experienced by seven females collectively. The response to the same death varied among individuals, however, and individuals exhibited different responses to the deaths of different herdmates (in 4 of 5 females that experienced multiple deaths). Two females showed increased cortisol and two showed decreased cortisol in response to death, and all four experienced a death in which they showed no adrenal GC response.

Observations of zoo and wild elephants exhibiting signs of distress or showing empathetic and helping behaviors towards dying and deceased conspecifics [23, 24] 
suggest that death, although a natural process, can be an emotionally challenging experience. Whether a death yields an adrenal GC response likely depends on the relationship to the individual, the strength of social bonds, and social support from conspecifics or human caretakers.

In the death of a female calf (1.4 years of age), both the mother and a herdmate showed significant decreases in cortisol in the short-term. The calf's death to elephant endotheliotropic herpesviruses was sudden, and there were no obvious signs in the days leading up to her death. Although the calf was still nursing, the mother had resumed cycling and showed no change in reproductive state after the calf's death. There were no obvious signs of depression based on keeper records in the days following the death. One possibility is that rearing of a calf, for both mother and allomother, is associated with an increase in "stressful" stimuli, either in terms of greater physiological (e.g., lactation) or physical and psychological (e.g., calf rearing) demands, so a sudden loss of that responsibility may lead to reduced cortisol. This plausible explanation is supported by findings that cortisol is highest during lactational anestrus. Although it is possible that lactation is a physiological stressor, the mother exhibited no prolonged elevations or depressions in cortisol concentrations during lactation, so it is unlikely the decreased cortisol after the death represented adrenal exhaustion.

\section{Transfers of herdmates}

Transfer of a herdmate was a significant predictor of mean cortisol concentration in $29 \%$ $(5 / 17)$ of the events experienced by seven females collectively, and all were in response 
to transfers of males; there were no significant effects in response to transfers of female herdmates.

The lack of adrenal GC response to transfers in of adult females in this study was surprising. Disrupting stable social groups by adding or removing individuals has been shown to cause social instability and increased aggression in some species $[95,96]$, resulting in elevated GC production [97]. In elephants, introductions of new females have resulted in increased GCs and behavioral changes in both residents and transfers [30, 31]. It is possible the quarantine and acclimation period after transfer was too long to register a response, and measurement surrounding the social introduction phase would have yielded a stronger response.

With the transfer out of a sub-adult male, both his mother and an unrelated female showed an increase in mean cortisol. A transfer out of a herdmate constitutes a sudden change that is not predictable, with no signals of impending departure (as perceived by resident animals) such as illness or changes in behavior - a change that is not part of the natural history for this species although it may be part of an individual's life history. In this case, the male transferred had reached puberty and would have begun to leave his natal group in the wild, and he was no longer socially integrated with the females. Although the departing individuals may no longer be socially integrated in the herd in daily life, these findings suggest that management practices should consider ways to prepare and acclimatize resident elephants after a herd mate is transferred out. 


\section{Transfer of self}

Transfer of self was a significant predictor of mean cortisol concentration in the three individuals with sufficient data to model, with two females showing higher, and one showing lower concentrations post-transfer. All involved transfers to NZP. Female F5NZ showed an increase only in the first 15 days post-transfer, indicating she acclimated quickly. Her transfer was a short distance and she was returning to her home facility after a year-long breeding loan, and into a herd in which she had existing social bonds, so it is not surprising she adjusted so quickly. The other two females were a mother and daughter that transferred together with one other herdmate from the same facility. The transfer was a longer distance requiring multiple days of travel. The daughter, F8NZ, acclimated quickly showing an increased cortisol concentration only in the first 30 days. The mother, F9NZ, took longer to acclimate, showing decreased cortisol concentration for at least 45 days post transfer.

Because of the quarantine time requirement (c.a. 30 days), any change in adrenal GC response for a transfer of self was associated with transport and acclimation rather than social introductions to resident elephants. Transportation has been recognized as a stressor in domestic and wild animals [27, 28], so it was not surprising that short-term increases were observed. Quick acclimation has been reported in working African elephants after translocation to a new reserve [26]. Response to transportation may differ with factors including prior crate training, travel distance, and physical condition. Furthermore, prior translocations and social relationships and interactions might have 
modified the stress response [13], perhaps explaining why two elephants showed an increase in cortisol, while one showed a decrease post-transfer.

\section{Death of self}

Four females were humanely euthanized during the study. The health decline leading to euthanasia was a significant predictor of mean cortisol concentration in one of two females with sufficient data to model. One female showed significantly higher mean cortisol concentration in the final 30 days of her life compared to the previous 30 days, with a sharp increase in the last week prior to euthanasia. By contrast, the other female showed no increases prior to euthanasia. It is possible that difference in responses could be attributed to the nature and intensity of the health decline and the point at which the euthanasia decision was made. Both suffered from chronic foot and joint problems that were exacerbated over time and became less responsive to anti-inflammatory treatments. In the period leading up to euthanasia, F6NZ increasingly leaned on objects/walls to relieve pain, whereas F4OZ's decline was more gradual and the decision to euthanize may have been made at a different point in decline.

\section{$\underline{\text { Individuality in adrenal GC response }}$}

Adrenal cortisol responsiveness clearly varied across individuals, with each being exposed to two to seven social life events. In six females with over 10 years of cortisol data, the percentage of significant responses to social life events ranged from 0 to $50 \%$. These extremes were observed in two females at NZP, each of whom experienced six social events with five events that were the same. Female F5NZ was the most responsive 
to deaths and transfers, whereas the oldest female in the study, F7NZ, did not show a significant response to any of the six events she experienced. Social relationships likely played a role in that the death and transfer out of a herdmate that yielded an effect were both offspring to F5NZ.

Individual variation in GC response to exogenous stimuli is ubiquitous. Cockrem [98] presents studies that show mean plasma cortisol concentrations increase in response to capture, restraint, or confinement in all vertebrate groups (fish, amphibians, reptiles, birds, mammals), but within each group there is extensive individual variation in response to the same stressor. In mammals, endogenous factors that lead to differences in GC responses include genetic variation and maternal influences before and after birth (reviewed in Cockrem [98] and Palme [6]).

Individuals have different personalities and temperaments or coping styles, and individual variation in GC response has been linked to coping styles described as proactive/bold and reactive/shy in laboratory and farm animals [99, 100]. Fanson et al. [27] found that individual patterns of adrenocortical activity in response to long-distance relocation of female Asian elephants were at least partially explained by differences in elephant behavioral traits or temperament. Grand et al. [101] found differences in cortisol response patterns to be correlated with fearful, effective, sociable, and aggressive personality components in a small group of captive African elephants. Temperament may have played a role in F7NZ's apparent lack of adrenal GC response to numerous life events. Caretakers described her as "a very wise elephant who socializes well with both 
elephants and people. She is careful and does not like change, but copes with it well by letting others experience things first, and then she will follow".

Aspects of social environment (group structure, degree of social integration or isolation, dominance rank) are important factors in how an individual or group responds to social events, and these factors have been shown to exert influence on the adrenal GC response in many social species $[89,90,102]$. Social support may offer protective or buffering effects against stressful challenges [103]. Increasing evidence suggests that affiliative behaviors in some animals can provide a buffer against stress and have a positive impact on measures of health and well-being [104]. The capacity for reassurance and empathy towards conspecifics in distress has been shown in Asian elephants [105]. Regardless of the physical location relative to conspecifics, elephants in this study could likely hear human activities surrounding major events and certainly any elephant vocalizations, but it is plausible that physical access or lack of access to herdmates giving birth or dying could have influenced adrenal GC response given the prevalence of tactile behaviors in affiliative interactions in this species. Degree of familiarity and stability of social bonds are factors influencing social support, but are not necessarily required [103]. Differences in social structure between African and Asian elephants may shed light on the observed responses, or lack thereof. African elephants form complex, multi-tiered social groups that are important to survival, whereas Asian herds are smaller and bonds are more fluid [106], thus the response to social disturbance in Asian elephants may not be as pronounced as in African elephants [107]. The influence of dominance rank on adrenal GC response was not measured because the rank of some individuals changed over time, 
and there were periods when dominance hierarchy was in flux, e.g., after the death or transfer of a dominant female. In particular, at NZP, the dominance hierarchy among the females was not linear with clear and consistent rankings. Thus, we saw no apparent evidence of differential responses based on social rank, and Proctor et al. [79] found no evidence of adrenal GC differences related to dominance status, at least not in African elephants.

Finally, individual differences in adrenal GC response to environmental change may be attributed in part to the human-animal relationships that allow each to make predictions about the other, and human-animal bonds that have been demonstrated in both stockmanship and zoo keeping [108, 109]. Zoo elephants are highly managed, and therefore caretakers are an important facet of elephants' daily lives. The manner in which caretakers look after their elephants and how elephants respond in preparation for or following potentially stressful events may influence an individual elephant's response to those events.

\section{$\underline{\text { Conclusion }}$}

This retrospective study furthers our understanding of the role of cortisol in ovarian function and emphasizes the importance of taking intrinsic patterns of cortisol secretion into account when assessing adrenal GC responses to external stimuli. Results also highlight the importance of routine blood sampling for long-term monitoring of reproductive and adrenal activity. While this study focused on cortisol as an indicator of adrenal function, measures of other adrenal hormones, like dehydroepiandrosterone 
(DHEA) and dopamine, in females across different reproductive states could shed light on additional intrinsic patterns and possible covariance with cortisol in relation to reproduction. The role of dopamine also should be explored due to its inhibitory control of prolactin and known relationship with ovarian dysfunction in African elephants [110]. Furthermore, age-related changes in DHEA and adrenal hormones could provide insight into the relationship between adrenal activity and metabolic changes, and aid in the care of geriatric elephants.

Because social life events can disrupt stable social groups and social bonds, it was surprising that less than $40 \%$ of social life events altered GC activity. However, changes in cortisol are only one measure of the stress response. Thus, an integrated approach using both behavioral and physiological measures is necessary to fully understand how animals perceive and interact with changes or challenges in their environment $[111,112]$. Further investigations could include examining the underlying mechanisms through which social behavior, including both animal-animal and human-animal interactions, may provide a buffer against such stressors. For example, measures of oxytocin, a neuropeptide known for promoting social behavior through which positive social interactions suppress the HPA axis (reviewed in DeVries et al. [104]), could clarify the role of prosocial behavior and social support in how individual elephants respond to stressors.

Indivduality in adrenal GC response to social life events provides evidence that life history, social relationships, temperament, and social support are important factors 
influencing the impact of perceived stressors. The observed response of females to transfers in and out of males reinforces the important role of males in the herd even if they are not always socially integrated. Pinto et al [113] concludes that even temporary integration of a male elephant into a female group in captivity has positive impacts in females, and that further studies should be done on the influence of males on welfare of females in captivity. Finally, an understanding of the impact of life events and resiliency to perceived stressors would be beneficial in determining the welfare needs of individuals and groups.

\section{Acknowledgements}

The existence of this vast dataset is attributed to over 40 elephant handlers at both the Oregon Zoo and National Zoological Park, who collected blood and/or urine samples from individuals of each herd for over 25 years (OZ: Jeb Barsh, Dimas Dominguez, Shawn Finnell, Pat Flora, Doug Groves, Jay Haight, Roger Henneous, Ray Hopper, David Illig, Mike Keele, Jeremy Kirby, Bob Lee, Fred Marion, Sioux Marion, Phil Prewett, Charlie Rutkowski, Joe Sabastiani, Jim Sanford, Pam Starkey, and April Yoder; NZP: Paige Babel, Tony Barthel, Heather Baskett, Lisa Belitz, Amanda Bobyack, Matt

Chambers, Ed Daniel, Debbie Flinkman, Debby Flynn, Ashley Fortner, Marie Galloway, Jason Gueguej, Brad Hange, Jourdon Hargis, John Lehnhardt, Ann Mrozinski, Andrea Pohlman, Belinda Reser, Rebecca Riley, Sean Royals, Rachada Sims, Kayleigh Sullivan, Rebecca Spickler.) Many thanks to Natalia Prado for help running the cortisol assay on Oregon Zoo samples. We owe the integrity of the National Zoo dataset to the 
Smithsonian Conservation Biological Institute Endocrinology Lab: Nicole Boisseau, Steve Paris, Nicole Parker, Natalia Prado, and Sue Walker. We appreciate the guidance of Deborah Lutterschmidt and Debbie Duffield of Portland State University in manuscript preparation.

\section{Literature Cited}

1. Kumar V, Umapathy G. Non-invasive monitoring of steroid hormones in wildlife for conservation and management of endangered species-A review. Indian J Exp Biol. 2019;57:307-14.

2. Brown JL. Comparative Reproductive Biology of Elephants. In: Holt WV, Brown JL, Comizzoli P, editors. Reproductive Sciences in Animal Conservation: Progress and Prospects. Advances in Experimental Medicine and Biology. New York, NY: Springer Science and Business Media; 2014. p. 135-69.

3. Brown JL. Reproductive endocrine monitoring of elephants: An essential tool for assisting captive management. Zoo Biol. 2000;19(5):347-67.

4. Möstl E, Palme R. Hormones as indicators of stress. Domestic Animal Endocrinology. 2002;23(1):67-74. doi: https://doi.org/10.1016/S0739-7240(02)00146-7.

5. Ralph CR, Tilbrook AJ. INVITED REVIEW: The usefulness of measuring glucocorticoids for assessing animal welfare. J Anim Sci. 2016;94(2):457-70. Epub 2016/04/12. doi: https://doi.org/10.2527/jas.2015-9645. PubMed PMID: 27065116.

6. Palme R. Non-invasive measurement of glucocorticoids: Advances and problems. Physiol Behav. 2019;199:229-43. Epub 2018/11/24. doi: https://doi.org/10.1016/j.physbeh.2018.11.021. PubMed PMID: 30468744.

7. Buwalda B, Scholte J, de Boer SF, Coppens CM, Koolhaas JM. The acute glucocorticoid stress response does not differentiate between rewarding and aversive social stimuli in rats. Horm Behav. 2012;61(2):218-26. Epub 2012/01/03. doi: https://doi.org/10.1016/j.yhbeh.2011.12.012. PubMed PMID: 22210197. 
8. Liptrap RM, Raeside JI. A relationship between plasma concentrations of testosterone and corticosteroids during sexual and aggressive behaviour in the boar. $\mathrm{J}$ Endocrinol. 1978;76(1):75-85. Epub 1978/01/01. doi: 10.1677/joe.0.0760075. PubMed PMID: 203644.

9. Wielebnowski N. Stress and distress: evaluating their impact for the well-being of zoo animals. Journal of the American Veterinary Medical Association. 2003;223(7):9737. doi: 10.2460/javma.2003.223.973.

10. McCormick SD, Romero LM. Conservation Endocrinology. BioScience. 2017;67(5):429-42. doi: 10.1093/biosci/bix026 \%J BioScience.

11. Yeates JW, Main DCJ. Assessment of positive welfare: A review. The Veterinary Journal. 2008;175(3):293-300. doi: https://doi.org/10.1016/j.tvj1.2007.05.009.

12. Busch DS, Hayward LS. Stress in a conservation context: A discussion of glucocorticoid actions and how levels change with conservation-relevant variables. Biol Conserv. 2009;142(12):2844-53. doi: https://doi.org/10.1016/j.biocon.2009.08.013.

13. Moberg GP. Biological response to stress: implications for animal welfare. Moberg GP, Mench JA, editors. New York, New York, USA.: CABI Pu; 2000.

14. Nelson RJ. An introduction to Behavioral Endocrinology. Fourth ed. Sunderland, Massachusetts: Sinauer Associates, Inc.; 2011.

15. Romero LM. Physiological stress in ecology: lessons from biomedical research. Trends Ecol Evol. 2004;19(5):249-55. Epub 2006/05/17. doi: https://doi.org/10.1016/j.tree.2004.03.008. PubMed PMID: 16701264.

16. Viljoen JJ, Ganswindt A, Palme R, Reynecke HC, Du Toit JT, Langbauer Jr WR. Measurement of concentrations of faecal glucocorticoid metabolites in free-ranging African elephants within the Kruger National Park Koedoe. 2008;50:18-21.

17. Foley C, Papageorge S, Wasser S. Noninvasive stress and reproductive measures of social and ecological pressures in free-ranging African elephants. Conserv Biol. 2001;15(4):1134-42. doi: https://doi.org/10.1046/j.1523-1739.2001.0150041134.x.

18. Ganswindt A, Münscher S, Henley M, Palme R, Thompson P, Bertschinger H. Concentrations of faecal glucocorticoid metabolites in physically injured free-ranging African elephants Loxodonta africana. Wildlife Biol. 2010;16(3):323-32. doi: https://doi.org/10.2981/09-081. 
19. Norkaew T, Brown JL, Bansiddhi P, Somgird C, Thitaram C, Punyapornwithaya $\mathrm{V}$, et al. Body condition and adrenal glucocorticoid activity affects metabolic marker and lipid profiles in captive female elephants in Thailand. PLoS One. 2018;13(10):e0204965. Epub 2018/10/03. doi: https://doi.org/10.1371/journal.pone.0204965. PubMed PMID: 30278087; PubMed Central PMCID: PMCPMC6168167.

20. Mumby HS, Mar KU, Thitaram C, Courtiol A, Towiboon P, Min-Oo Z, et al. Stress and body condition are associated with climate and demography in Asian elephants. Conserv Physiol. 2015;3(1):cov030. Epub 2015/01/01. doi: https://doi.org/10.1093/conphys/cov030. PubMed PMID: 27293715; PubMed Central PMCID: PMCPMC4778474.

21. Whilde J, Marples N. Effect of a birth on the behavior of a family group of Asian elephants (Elephas maximus) at Dublin Zoo. Zoo Biol. 2012;31(4):442-52. Epub 2011/07/29. doi: https://doi.org/10.1002/zoo.20408. PubMed PMID: 21796671.

22. Lee PC. Allomothering among African elephants. Anim Behav. 1987;35:278-91. doi: https://doi.org/10.1016/S0003-3472(87)80234-8.

23. Douglas-Hamilton I, Bhalla S, Wittemyer G, Vollrath F. Behavioural reactions of elephants towards a dying and deceased matriarch. Appl Anim Behav Sci. 2006;100(1):87-102. doi: https://doi.org/10.1016/j.applanim.2006.04.014.

24. Sharma N, Pokharel SS, Kohshima S, Sukumar R. Behavioural responses of freeranging Asian elephants (Elephas maximus) towards dying and dead conspecifics. Primates. 2020;61(1):129-38. Epub 2019/08/21. doi: 10.1007/s10329-019-00739-8. PubMed PMID: 31428950.

25. Gobush KS, Mutayoba BM, Wasser SK. Long-term impacts of poaching on relatedness, stress physiology, and reproductive output of adult female african elephants. Conserv Biol. 2008;22(6):1590-9. Epub 2008/09/02. doi: 10.1111/j.15231739.2008.01035.x. PubMed PMID: 18759771.

26. Millspaugh JJ, Burke T, DYK G, Slotow R, Washburn BE, Woods RJ. Stress response of working African elephants to transportation and safari adventures. J Wildl Manage. 2007;71(4):1257-60. doi: https://doi.org/10.2193/2006-015.

27. Fanson KV, Lynch M, Vogelnest L, Miller G, Keeley T. Response to longdistance relocation in Asian elephants (Elephas maximus): monitoring adrenocortical activity via serum, urine, and feces. Eur J Wildl Res. 2013;59(5):655-64. doi: https://doi.org/10.1007/s10344-013-0718-7. 
28. Laws N, Ganswindt A, Heistermann M, Harris M, Harris S, Sherwin C. A Case Study: Fecal Corticosteroid and Behavior as Indicators of Welfare During Relocation of an Asian Elephant. J Appl Anim Welf Sci. 2007;10(4):349-58. Epub 2007/11/01. doi: https://doi.org/10.1080/10888700701555600. PubMed PMID: 17970634.

29. Viljoen JJ, Ganswindt A, Du Toit JT, Langbauer Jr WR. Translocation stress and faecal glucocorticoid metabolite levels in free-ranging African savanna elephants. Afr J Wildl Res. 2008;38(2):146-52.

30. Schmid J, Heistermann M, Gansloßer U, Hodges J. Introduction of foreign female Asian elephants (Elephas maximus) into an existing group: behavioural reactions and changes in cortisol levels. Anim Welf. 2001;10(4):357-72.

31. Burks KD, Mellen JD, Miller GW, Lehnhardt J, Weiss A, Figueredo AJ, et al. Comparison of two introduction methods for African elephants (Loxodonta africana). Zoo Biol. 2004;23(2):109-26. doi: https://doi.org/10.1002/zoo.10132.

32. Brown JL, Kersey DC, Freeman EW, Wagener T. Assessment of diurnal urinary cortisol excretion in Asian and African elephants using different endocrine methods. Zoo Biol. 2010;29(2):274-83. Epub 2009/08/12. doi: https://doi.org/10.1002/zoo.20268. PubMed PMID: 19670316.

33. Casares M, Silván G, Carbonell MD, Gerique C, Martinez-Fernandez L, Cáceres $\mathrm{S}$, et al. Circadian rhythm of salivary cortisol secretion in female zoo-kept African elephants (Loxodonta africana). Zoo Biology. 2016;35(1):65-9. doi: 10.1002/zoo.21262.

34. Menargues A, Urios V, Limiñana R, Mauri M. Circadian Rhythm of Salivary Cortisol in Asian Elephants (Elephas maximus): A Factor to Consider During Welfare Assessment. J Appl Anim Welf Sci. 2012;15(4):383-90. doi: 10.1080/10888705.2012.709157.

35. Hambrecht S, Oerke A-K, Heistermann M, Dierkes PW. Diurnal variation of salivary cortisol in captive African elephants (Loxodonta africana) under routine management conditions and in relation to a translocation event. Zoo Biology. n/a(n/a). doi: 10.1002/zoo.21537.

36. Menargues Marcilla A, Urios V, Limiñana R. Seasonal rhythms of salivary cortisol secretion in captive Asian elephants (Elephas maximus). Gen Comp Endocrinol. 2012;176(2):259-64. doi: https://doi.org/10.1016/j.ygcen.2012.02.001. 
37. Fanson KV, Keeley T, Fanson BG. Cyclic changes in cortisol across the estrous cycle in parous and nulliparous Asian elephants. Endocr Connect. 2014;3(2):57-66. Epub 2014/03/14. doi: https://doi.org/10.1530/EC-14-0025. PubMed PMID: 24623735; PubMed Central PMCID: PMCPMC3987288.

38. Brown JL, Somerville M, Riddle HS, Keele M, Duer CK, Freeman EW. Comparative endocrinology of testicular, adrenal and thyroid function in captive Asian and African elephant bulls. Gen Comp Endocrinol. 2007;151(2):153-62. Epub 2007/03/06. doi: http://dx.doi.org/10.1016/j.ygcen.2007.01.006. PubMed PMID: 17336304 .

39. Chave E, Edwards KL, Paris S, Prado N, Morfeld KA, Brown JL. Variation in metabolic factors and gonadal, pituitary, thyroid, and adrenal hormones in association with musth in African and Asian elephant bulls. Gen Comp Endocrinol. 2019;276:1-13. doi: https://doi.org/10.1016/j.ygcen.2019.02.005.

40. Kajaysri J, Nokkaew W. Assessment of pregnancy status of Asian elephants (Elephas maximus) by measurement of progestagen and glucocorticoid and their metabolite concentrations in serum and feces, using enzyme immunoassay (EIA). J Vet Med Sci. 2014;76(3):363-8. Epub 2013/11/22. doi: https://doi.org/10.1292/jvms.13-0103. PubMed PMID: 24257195; PubMed Central PMCID: PMCPMC4013362.

41. Brown JL, Lehnhardt J. Serum and urinary hormones during pregnancy and the peri- and postpartum period in an Asian elephant (Elephas, maximus). Zoo Biol. 1995;14(6):555-64. doi: https://doi.org/10.1002/zoo.1430140608.

42. Liptrap R, Raeside J. Effect of corticotrophin and corticosteroids on plasma interstitial cell-stimulating hormone and urinary steroids in the boar. J Endocrinol. 1968;42(1):33-43.

43. Howland BE, Sanford LM, Palmer WM. Changes in serum levels of LH, FSH, prolactin, testosterone, and cortisol associated with season and mating in male pygmy goats. J Androl. 1985;6(2):89-96. Epub 1985/03/01. doi: 10.1002/j.19394640.1985.tb00822.x. PubMed PMID: 3921507.

44. Borg KE, Esbenshade KL, Johnson BH. Cortisol, growth hormone, and testosterone concentrations during mating behavior in the bull and boar. J Anim Sci. 1991;69(8):3230-40. doi: 10.2527/1991.6983230x \%J Journal of Animal Science.

45. Colborn DR, Thompson DL, Jr., Roth TL, Capehart JS, White KL. Responses of cortisol and prolactin to sexual excitement and stress in stallions and geldings. J Anim Sci. 1991;69(6):2556-62. doi: 10.2527/1991.6962556x \%J Journal of Animal Science. 
46. Turner AI, Rivalland ETA, Clarke IJ, Tilbrook AJ. Stressor Specificity of Sex Differences in Hypothalamo-Pituitary-Adrenal Axis Activity: Cortisol Responses to Exercise, Endotoxin, Wetting, and Isolation/Restraint Stress in Gonadectomized Male and Female Sheep. Endocrinology. 2010;151(9):4324-31. doi: 10.1210/en.2010-0234 \%J Endocrinology.

47. Boedeker NC, Hayek LA, Murray S, de Avila DM, Brown JL. Effects of a gonadotropin-releasing hormone vaccine on ovarian cyclicity and uterine morphology of an Asian elephant (Elephas maximus). J Zoo Wildl Med. 2012;43(3):603-14. Epub 2012/10/23. doi: https://doi.org/10.1638/2011-0270.1. PubMed PMID: 23082526.

48. Edwards KL, Bansiddhi P, Paris S, Galloway M, Brown JL. The development of an immunoassay to measure immunoglobulin A in Asian elephant feces, saliva, urine and serum as a potential biomarker of well-being. Conserv Physiol. 2019;7(1):coy077. Epub 2019/03/25. doi: https://doi.org/10.1093/conphys/coy077. PubMed PMID: 30906557; PubMed Central PMCID: PMCPMC6425258.

49. Brown JL, Wemmer CM, Lehnhardt J. Urinary cortisol analysis for monitoring adrenal activity in elephants. Zoo Biol. 1995;14(6):533-42. doi: https://doi.org/10.1002/zoo.1430140606.

50. Young KM, Walker SL, Lanthier C, Waddell WT, Monfort SL, Brown JL. Noninvasive monitoring of adrenocortical activity in carnivores by fecal glucocorticoid analyses. Gen Comp Endocrinol. 2004;137(2):148-65. Epub 2004/05/26. doi: http://dx.doi.org/10.1016/j.ygcen.2004.02.016. PubMed PMID: 15158127.

51. Brown JL, Walker SL, Moeller T. Comparative endocrinology of cycling and non-cycling Asian (Elephas maximus) and African (Loxodonta africana) elephants. Gen Comp Endocrinol. 2004;136(3):360-70. Epub 2004/04/15. doi: https://doi.org/10.1016/j.ygcen.2004.01.013. PubMed PMID: 15081836.

52. Brown JL, Göritz F, Pratt-Hawkes N, Hermes R, Galloway M, Graham LH, et al. Successful artificial insemination of an Asian elephant at the National Zoological Park. Zoo Biol. 2004;23(1):45-63. doi: https://doi.org/10.1002/zoo.10116.

53. Rolland RM, Hunt KE, Kraus SD, Wasser SK. Assessing reproductive status of right whales (Eubalaena glacialis) using fecal hormone metabolites. Gen Comp Endocrinol. 2005;142(3):308-17. Epub 2005/06/07. doi: https://doi.org/10.1016/j.ygcen.2005.02.002. PubMed PMID: 15935157. 
54. Monfort SL, Wemmer C, Kepler TH, Bush M, Brown JL, Wildt DE. Monitoring ovarian function and pregnancy in Eld's deer (Cervus eldi thamin) by evaluating urinary steroid metabolite excretion. J Reprod Fertil. 1990;88(1):271-81. Epub 1990/01/01. doi: https://doi.org/10.1530/jrf.0.0880271. PubMed PMID: 2156072.

55. Glaeser SS, Hunt KE, Martin MS, Finnegan M, Brown JL. Investigation of individual and group variability in estrous cycle characteristics in female Asian elephants (Elephas maximus) at the Oregon Zoo. Theriogenology. 2012;78(2):285-96. Epub 2012/05/15. doi: http://dx.doi.org/10.1016/j.theriogenology.2012.01.014. PubMed PMID: 22578614.

56. R Core Team. R: A language and environment for statistical computing. Vienna, Austria: R Foundation for Statistical Computing; Available from: https://www.rproject.org/; 2020.

57. Fanson B, Fanson K. Hormlong: Longitudinal Analysis of Hormone Data. R package version 1.0. 2014.

58. Brown JL, Schmitt DL, Bellem A, Graham LH, Lehnhardt J. Hormone Secretion in the Asian Elephant (Elephas maximus): Characterization of Ovulatory and Anovulatory Luteinizing Hormone Surges. Biol Reprod. 1999;61(5):1294-9. Epub 1999/10/21. doi: https://doi.org/10.1095/biolreprod61.5.1294. PubMed PMID: 10529277.

59. Brown JL, Lehnhardt J. Secretory patterns of serum prolactin in Asian (Elephas maximus) and African (Loxodonta africana) elephants during different reproductive states: Comparison with concentrations in a noncycling African elephant. Zoo Biol. 1997;16(2):149-59.

60. Lee B. AZA Regional Studbook for Asian Elephant (Elephas maximus). Portland, OR: Association of Zoos and Aquariums, 2019 09/24/2019. Report No.

61. Brown MB, Forsythe AB. Robust Tests for the Equality of Variances. J Am Stat Assoc. 1974;69(346):364-7. doi: https://doi.org/10.1080/01621459.1974.10482955.

62. Dayton A, Exner EC, Bukowy JD, Stodola TJ, Kurth T, Skelton M, et al. Breaking the Cycle: Estrous Variation Does Not Require Increased Sample Size in the Study of Female Rats. Hypertension. 2016;68(5):1139-44. Epub 2016/09/28. doi: https://doi.org/10.1161/HYPERTENSIONAHA.116.08207. PubMed PMID: 27672030; PubMed Central PMCID: PMCPMC5104284.

63. Dag O, Dolgun A, Konar NM. onewaytests: An R Package for One-Way Tests in Independent Groups Designs. The R Journal 2018;10:175-99. doi: Available from: https://journal.r-project.org/archive/2018/RJ-2018-022/index.html. 
64. Rasbash J, Charlton C, Browne W, Healy M, Cameron B. MLwiN v 2.02. Bristol, United Kingdom: University of Bristol: Centre for Multilevel Modelling; Available from: http://www.bristol.ac.uk/cmm/software/mlwin/; 2005.

65. Bolker BM, Brooks ME, Clark CJ, Geange SW, Poulsen JR, Stevens MHH, et al. Generalized linear mixed models: a practical guide for ecology and evolution. Trends Ecol Evol. 2009;24(3):127-35. Epub 2009/02/03. doi: https://doi.org/10.1016/j.tree.2008.10.008. PubMed PMID: 19185386.

66. Harrison XA, Donaldson L, Correa-Cano ME, Evans J, Fisher DN, Goodwin CED, et al. A brief introduction to mixed effects modelling and multi-model inference in ecology. PeerJ. 2018;6:e4794. doi: 10.7717/peerj.4794.

67. Edwards KL, Shultz S, Pilgrim M, Walker SL. Irregular ovarian activity, body condition and behavioural differences are associated with reproductive success in female eastern black rhinoceros (Diceros bicornis michaeli). Gen Comp Endocrinol. 2015;214:186-94. Epub 2014/08/26. doi: https://doi.org/10.1016/j.ygcen.2014.07.026. PubMed PMID: 25150145.

68. Edwards KL, Trotter J, Jones M, Brown JL, Steinmetz HW, Walker SL. Investigating temporary acyclicity in a captive group of Asian elephants (Elephas maximus): Relationship between management, adrenal activity and social factors. Gen Comp Endocrinol. 2016;225:104-16. doi: http://dx.doi.org/10.1016/j.ygcen.2015.09.011.

69. Pinter-Wollman N, Isbell LA, Hart LA. Assessing translocation outcome: Comparing behavioral and physiological aspects of translocated and resident African elephants (Loxodonta africana). Biol Conserv. 2009;142(5):1116-24. doi: http://dx.doi.org/10.1016/j.biocon.2009.01.027.

70. Burke T, Page B, Van Dyk G, Millspaugh J, Slotow R. Risk and ethical concerns of hunting male elephant: behavioural and physiological assays of the remaining elephants. PLoS One. 2008;3(6):e2417. Epub 2008/06/19. doi: https://doi.org/10.1371/journal.pone.0002417. PubMed PMID: 18560517; PubMed Central PMCID: PMCPMC2426916.

71. Thitaram C, Brown JL. Monitoring and controlling ovarian activity in elephants. Theriogenology. 2018;109:42-7. Epub 2017/12/31. doi: https://doi.org/10.1016/j.theriogenology.2017.12.009. PubMed PMID: 29287954.

72. Atkinson HC, Waddell BJ. Circadian variation in basal plasma corticosterone and adrenocorticotropin in the rat: sexual dimorphism and changes across the estrous cycle. Endocrinology. 1997;138(9):3842-8. Epub 1997/09/01. doi: https://doi.org/10.1210/endo.138.9.5395. PubMed PMID: 9275073. 
73. Kersey DC, Wildt DE, Brown JL, Snyder RJ, Huang Y, Monfort SL. Rising fecal glucocorticoid concentrations track reproductive activity in the female giant panda (Ailuropoda melanoleuca). Gen Comp Endocrinol. 2011;173(2):364-70. Epub 2011/07/06. doi: https://doi.org/10.1016/j.ygcen.2011.06.013. PubMed PMID: 21726558.

74. Sosa C, Forcada F, Meikle A, Abecia JA. Increase in ovine plasma cortisol at oestrus and its relation with the metabolic status during the sexual cycle in sheep. Biol Rhythm Res. 2013;44(3):445-9. doi: https://doi.org/10.1080/09291016.2012.704793.

75. Schiml PA, Rissman EF. Cortisol facilitates induction of sexual behavior in the female musk shrew (Suncus murinus). Behav Neurosci. 1999;113(1):166-75. Epub 1999/04/10. doi: https://doi.org/10.1037/0735-7044.113.1.166. PubMed PMID: 10197916.

76. Edwards KL, Pilgrim M, Brown JL, Walker SL. Irregular Ovarian Cyclicity is Associated with Adrenal Activity in Female Eastern Black Rhinoceros (Diceros bicornis michaeli). Gen Comp Endocrinol. 2019:113376. Epub 2019/12/28. doi: https://doi.org/10.1016/j.ygcen.2019.113376. PubMed PMID: 31881202.

77. Tetsuka M. Actions of glucocorticoid and their regulatory mechanisms in the ovary. Anim Sci J. 2007;78(2):112-20. doi: https://doi.org/10.1111/j.17400929.2007.00414.x.

78. Proctor C, Brown J. Influence of Handling Method on Adrenal Activity in Zoo African and Asian Elephants. J Zoo Aquar Res. 2015;3:1-.

79. Proctor CM, Freeman EW, Brown JL. Influence of dominance status on adrenal activity and ovarian cyclicity status in captive African elephants. Zoo Biol. 2010;29(2):168-78. Epub 2009/12/25. doi: https://doi.org/10.1002/zoo.20292. PubMed PMID: 20033989.

80. Cavigelli SA, Monfort SL, Whitney TK, Mechref YS, Novotny M, McClintock MK. Frequent serial fecal corticoid measures from rats reflect circadian and ovarian corticosterone rhythms. J Endocrinol. 2005;184(1):153-63. Epub 2005/01/12. doi: https://doi.org/10.1677/joe.1.05935. PubMed PMID: 15642792.

81. Edwards PD, Boonstra R. Glucocorticoids and CBG during pregnancy in mammals: diversity, pattern, and function. Gen Comp Endocrinol. 2018;259:122-30. Epub 2017/11/21. doi: https://doi.org/10.1016/j.ygcen.2017.11.012. PubMed PMID: 29155262. 
82. Meyer JM, Walker SL, Freeman EW, Steinetz BG, Brown JL. Species and fetal gender effects on the endocrinology of pregnancy in elephants. Gen Comp Endocrinol. 2004;138(3):263-70. Epub 2004/09/15. doi: 10.1016/j.ygcen.2004.06.010. PubMed PMID: 15364209.

83. Kaewmanee S, Watanabe G, Yamamoto Y, Yamamoto T, Kishimoto M, Nagaoka $\mathrm{K}$, et al. Updated Reproductive Hormonal Profiles in Female Elephants. Thai Journal of Veterinary Medicine. 2011;41:91-4.

84. Pesántez-Pacheco JL, Heras-Molina A, Torres-Rovira L, Sanz-Fernández MV, García-Contreras C, Vázquez-Gómez M, et al. Maternal Metabolic Demands Caused by Pregnancy and Lactation: Association with Productivity and Offspring Phenotype in High-Yielding Dairy Ewes. Animals. 2019;9(6):295. doi: https://doi.org/10.3390/ani9060295. PubMed PMID: doi:10.3390/ani9060295.

85. Goymann W, East ML, Wachter B, Honer OP, Mostl E, Van't Hof TJ, et al. Social, state-dependent and environmental modulation of faecal corticosteroid levels in free-ranging female spotted hyenas. Proc Biol Sci. 2001;268(1484):2453-9. Epub 2001/12/19. doi: 10.1098/rspb.2001.1828. PubMed PMID: 11747563; PubMed Central PMCID: PMCPMC1088899.

86. Pokharel SS, Seshagiri PB, Sukumar R. Influence of the number of calves and lactating adult females in a herd on the adrenocortical activity of free-ranging Asian elephants. Wildlife Research. 2019;46(8):679-89. doi: 10.1071/wr18163.

87. Prado-Oviedo NA, Bonaparte-Saller MK, Malloy EJ, Meehan CL, Mench JA, Carlstead K, et al. Evaluation of Demographics and Social Life Events of Asian (Elephas maximus) and African Elephants (Loxodonta africana) in North American Zoos. PLoS One. 2016;11(7):e0154750. Epub 2016/07/16. doi: https://doi.org/10.1371/journal.pone.0154750. PubMed PMID: 27415437; PubMed Central PMCID: PMCPMC4945030.

88. McEwen BS, Wingfield JC. The concept of allostasis in biology and biomedicine. Horm Behav. 2003;43(1):2-15. Epub 2003/03/05. doi: https://doi.org/10.1016/S0018506X(02)00024-7. PubMed PMID: 12614627.

89. Creel S, Dantzer B, Goymann W, Rubenstein DR. The ecology of stress: effects of the social environment. Funct Ecol. 2013;27(1):66-80. doi: https://doi.org/10.1111/j.1365-2435.2012.02029.x. 
90. Hawkley LC, Cole SW, Capitanio JP, Norman GJ, Cacioppo JT. Effects of social isolation on glucocorticoid regulation in social mammals. Horm Behav. 2012;62(3):31423. Epub 2012/06/06. doi: https://doi.org/10.1016/j.yhbeh.2012.05.011. PubMed PMID: 22663934; PubMed Central PMCID: PMCPMC3449017.

91. Fernando P, Vidya TNC, Payne J, Stuewe M, Davison G, Alfred RJ, et al. DNA analysis indicates that Asian elephants are native to Borneo and are therefore a high priority for conservation. Plos Biol. 2003;1(1):110-5. doi: https://doi.org/10.1371/journal.pbio.0000006. PubMed PMID: ISI:000188834700018.

92. Sharma R, Goossens B, Heller R, Rasteiro R, Othman N, Bruford MW, et al. Genetic analyses favour an ancient and natural origin of elephants on Borneo. Sci Rep. 2018;8(1):880. doi: https://doi.org/10.1038/s41598-017-17042-5.

93. Garai M, Kurt F. The importance of socialisation to the well being of elephants. Zeitschrift des Kölner Zoo. 2006;2(49):85-102.

94. Schulte BA. Social structure and helping behavior in captive elephants. Zoo Biol. 2000;19(5):447-59.

95. Baranyi J, Bakos N, Haller J. Social instability in female rats: The relationship between stress-related and anxiety-like consequences. Physiol Behav. 2005;84(4):511-8. Epub 2005/04/07. doi: https://doi.org/10.1016/j.physbeh.2005.01.005. PubMed PMID: 15811385.

96. Coutellier L, Arnould C, Boissy A, Orgeur P, Prunier A, Veissier I, et al. Pig's responses to repeated social regrouping and relocation during the growing-finishing period. Appl Anim Behav Sci. 2007;105(1):102-14. doi:

https://doi.org/10.1016/j.applanim.2006.05.007.

97. Haller J, Fuchs E, Halász J, Makara GB. Defeat is a major stressor in males while social instability is stressful mainly in females: towards the development of a social stress model in female rats. Brain Res Bull. 1999;50(1):33-9. doi: https://doi.org/10.1016/S0361-9230(99)00087-8.

98. Cockrem JF. Individual variation in glucocorticoid stress responses in animals. Gen Comp Endocrinol. 2013;181:45-58. Epub 2013/01/10. doi: https://doi.org/10.1016/j.ygcen.2012.11.025. PubMed PMID: 23298571.

99. Koolhaas JM, Korte SM, De Boer SF, Van Der Vegt BJ, Van Reenen CG, Hopster H, et al. Coping styles in animals: current status in behavior and stressphysiology. Neurosci Biobehav Rev. 1999;23(7):925-35. Epub 1999/12/02. doi: https://doi.org/10.1016/S0149-7634(99)00026-3. PubMed PMID: 10580307. 
100. Hovland AL, Rød AMS, Eriksen MS, Palme R, Nordgreen J, Mason GJ. Faecal cortisol metabolites as an indicator of adrenocortical activity in farmed silver foxes (Vulpes vulpes). Appl Anim Behav Sci. 2017;197:75-80. doi:

https://doi.org/10.1016/j.applanim.2017.08.009.

101. Grand AP, Kuhar CW, Leighty KA, Bettinger TL, Laudenslager ML. Using personality ratings and cortisol to characterize individual differences in African Elephants (Loxodonta africana). Appl Anim Behav Sci. 2012;142(1-2):69-75. doi:

https://doi.org/10.1016/j.applanim.2012.09.002.

102. Stocker M, Munteanu A, Stöwe M, Schwab C, Palme R, Bugnyar T. Loner or socializer? Ravens' adrenocortical response to individual separation depends on social integration. Horm Behav. 2016;78:194-9. Epub 2015/12/04. doi: https://doi.org/10.1016/j.yhbeh.2015.11.009. PubMed PMID: 26631484; PubMed Central PMCID: PMCPMC4754940.

103. Rault J-L. Friends with benefits: social support and its relevance for farm animal welfare. Appl Anim Behav Sci. 2012;136(1):1-14. doi: https://doi.org/10.1016/j.applanim.2011.10.002.

104. DeVries AC, Glasper ER, Detillion CE. Social modulation of stress responses. Physiol Behav. 2003;79(3):399-407. Epub 2003/09/05. doi:

https://doi.org/10.1016/S0031-9384(03)00152-5. PubMed PMID: 12954434.

105. Plotnik JM, de Waal FBM. Asian elephants (Elephas maximus) reassure others in distress. PeerJ. 2014;2:e278. Epub 2014/04/02. doi: https://doi.org/10.7717/peerj.278. PubMed PMID: 24688856; PubMed Central PMCID: PMCPMC3932735.

106. de Silva S, Wittemyer G. A Comparison of Social Organization in Asian Elephants and African Savannah Elephants. Int J Primatol. 2012;33(5):1125-41. doi: https://doi.org/10.1007/s10764-011-9564-1.

107. Brown JL, Carlstead K, Bray JD, Dickey D, Farin C, Ange-van Heugten K. Individual and environmental risk factors associated with fecal glucocorticoid metabolite concentrations in zoo-housed Asian and African elephants. PLoS One.

2019;14(9):e0217326. Epub 2019/09/05. doi:

https://doi.org/10.1371/journal.pone.0217326. PubMed PMID: 31483790; PubMed Central PMCID: PMCPMC6726191.

108. Carlstead K, Paris S, Brown JL. Good keeper-elephant relationships in North American zoos are mutually beneficial to welfare. Appl Anim Behav Sci. 2019;211:10311. doi: https://doi.org/10.1016/j.applanim.2018.11.003. 
109. Cole J, Fraser D. Zoo Animal Welfare: The Human Dimension. J Appl Anim Welf Sci. 2018;21(sup1):49-58. Epub 2018/10/17. doi:

https://doi.org/10.1080/10888705.2018.1513839. PubMed PMID: 30325229.

110. Brown JL, Paris S, Prado-Oviedo NA, Meehan CL, Hogan JN, Morfeld KA, et al. Reproductive Health Assessment of Female Elephants in North American Zoos and Association of Husbandry Practices with Reproductive Dysfunction in African Elephants (Loxodonta africana). PLoS One. 2016;11(7):e0145673. doi:

10.1371/journal.pone.0145673.

111. MacDougall-Shackleton SA, Bonier F, Romero LM, Moore IT. Glucocorticoids and "Stress" Are Not Synonymous. Integrative Organismal Biology. 2019;1(1). doi: https://doi.org/10.1093/iob/obz017.

112. Shepherdson D, Carlstead K, Wielebnowski N. Cross-institutional assessment of stress responses in zoo animals using longitudinal monitoring of faecal corticoids and behaviour. Anim Welf. 2004;13:S105-S14.

113. Pinto A, Stelvig M, Costa C, Colaço J, Colaço B. Influence of male presence on female Asian elephants (Elephas maximus) behaviour in captivity. Journal of Zoo and Aquarium Research. 2020;8(1):45-9. 
CHAPTER 3: Adrenal glucocorticoid activity associated with musth, aging, social changes, and medical treatment in adult male Asian elephants (Elephas maximus)

Sharon S. Glaeser ${ }^{1,}$, Stephen Paris ${ }^{2}$, Katie Edwards ${ }^{3}$, Candace Scarlatta ${ }^{1}$, Janine L. Brown ${ }^{2}$

1 Oregon Zoo, 4001 SW Canyon Rd, Portland, Oregon, 97221, United States of America, 2 Center for Species Survival, Smithsonian Conservation Biology Institute, Smithsonian National Zoological Park, Front Royal, Virginia, United States of America, 3 North of England Zoological Society, Chester Zoo, Upton-by-Chester, United Kingdom

* Sharon.Glaeser@oregonzoo.org

Keywords: Asian Elephant, Elephas maximus, adrenal activity, adrenocortical activity, cortisol, glucocorticoids, testosterone, endocrinology, hormones, musth, long-term variation, individual variation, animal welfare 


\begin{abstract}
Musth is a physiological and behavioral phenomenon in male elephants characterized by increased testosterone, heightened aggression and sexual behavior, and therefore can pose some unique challenges for human safety and animal welfare. This study characterized longitudinal patterns (9 to 22 years) of circulating testosterone and cortisol in four adult bulls spanning an age range of 12 to 54 years in relation to age and musth, and assessed their adrenal responses to social changes, tuberculosis (TB) treatment, and health declines. Circulating cortisol covaried positively with testosterone and musth, highlighting intrinsic patterns that should be considered when evaluating the impact of social or environmental change on adrenal glucocorticoid (GC) activity. Testosterone secretion decreased with advancing age in bulls after 31-40 years of age, whereas agerelated changes in cortisol were inconsistent and varied across individuals during musth versus inter-musth periods. By contrast, social changes involving the addition of a new male and the death of male herdmates had no apparent impact on mean cortisol concentrations in resident bulls. Increases in cortisol secretion were associated with activation of Mycobacterium tuberculosis disease in only one bull, suggesting cortisol does not provide a good biomarker for detecting the transition from latent to active TB. An increase in serum cortisol within 30 days after starting anti-TB therapy in one individual, and a decrease after ending therapy within 30 days shows the adrenal response to treatment was quick. This study highlights the importance of longitudinal hormone monitoring to track changes in physiological function and responses to social, health and
\end{abstract}


environmental change, which consequently can lead to more informed management decisions. 


\section{Introduction}

Musth is a physiological and behavioral phenomenon characterized by increased testosterone, heightened aggression or unpredictability, increased sexual behavior, and a temporary rise in dominance in both wild and captive Asian (Eisenberg et al., 1971; Jainudeen et al., 1972a; Rasmussen et al., 1984; Cooper et al., 1990; Lincoln and Ratnasooriya, 1996) and African (Poole and Moss, 1981; Hall-Martin and Van der Walt, 1984; Poole, 1987, 1989a, b; Cooper et al., 1990) elephant bulls. Bulls advertise musth status through a variety of chemicals exuded in temporal gland secretions and urine (Rasmussen et al., 1984; Rasmussen et al., 1990; Rasmussen et al., 2002; Scott, 2002), and secretions from the paired facial temporal glands and dribbling of urine from the penile prepuce characterize physical signs of musth (Jainudeen et al., 1972a; Jainudeen et al., 1972b; Poole and Moss, 1981; Hall-Martin and Van der Walt, 1984; Rasmussen et al., 1984; Poole, 1987; Rasmussen et al., 2002; Scott, 2002; Somgird et al., 2016). Previous studies of musth in captive Asian bulls showed onset of temporal gland secretions and urine dribbling occurred as testosterone began to increase, or within a few weeks thereafter (Lincoln and Ratnasooriya, 1996; Brown et al., 2007). Nutrition is an important factor in the expression of musth, and in both wild and captive elephants, musth generally terminates with a significant decline in body condition (Jainudeen et al., 1972b; Poole, 1989a). Musth in Asian bull elephants normally occurs annually or biannually during approximately the same time each year for an individual, and varies among bulls in both duration and months of occurrence (Jainudeen et al., 1972a). However, instances of irregular or near continuous musth-like symptoms have been observed in zoo-held bulls, 
perhaps due to lack of social suppression by other bulls or due to a consistently high level of nutrition (Brown et al., 2007). From a management perspective, because aggression often is associated with musth, it can create human safety and animal welfare concerns under certain circumstances. It is therefore important to better understand the physiological changes associated with musth, the factors influencing the expression of musth, and to have tools available to properly assess the musth condition and the wellbeing of male elephants of all ages.

Measures of glucocorticoids (GCs) are important for understanding how individuals respond to challenges or changes in their environment. Glucocorticoids (GCs) are secreted from the adrenal cortex in response to stimuli, moderating subsequent physiological processes, both beneficial and detrimental (Möstl and Palme, 2002; Buwalda et al., 2012; Ralph and Tilbrook, 2016; Palme, 2019). The primary role of GCs is basic energy regulation; however, under stressful conditions, they facilitate physiological changes and are important mediators of coping (Busch and Hayward, 2009). In zoo settings, increased GCs in elephants have been observed in response to the opening of a new zoo (Menargues et al., 2008), during flooring renovations (Boyle et al., 2015), and introduction of an unfamiliar conspecific (Dathe et al., 1992; Schmid et al., 2001; Burks et al., 2004). While not reproductive hormones per se, GC elevations in elephants are also associated with normal physiological states such as musth (Brown et al., 2007; Yon et al., 2007; Chave et al., 2019), as well as pregnancy and parturition (Brown and Lehnhardt, 1995; Kajaysri and Nokkaew, 2014). In males of other species, GCs increase during rut and in association with mating behavior (Liptrap and Raeside, 
1968; Liptrap and Raeside, 1978; Howland et al., 1985; Borg et al., 1991; Mooring et al., 2006), as well as in association with aggressive behavior and dominance in some species [reviewed by Sands and Creel (2004)].

In long-lived social species like elephants, age-related and social factors are also important considerations for species management. It is now recognized that wild bull elephants have complex social structures and relationships (Evans and Harris, 2008; Goldenberg et al., 2014; Keerthipriya et al., 2018). Hartley et al. (2019) reports relatively low rates of musth expression in elephant bulls in European and North American zoos, perhaps because the vast majority are under 20 years of age. Furthermore, many of those bulls have restricted opportunities for social interactions, either with females or other bulls. Ganswindt et al. (2010) hypothesized that for wild African elephants, associations with non-musth bulls may have a social buffering effect on musth bulls because concentrations of fecal GCs were lower in musth bulls in the presence of non-musth bulls as compared to when they were alone. So, perhaps the housing of multiple bulls is beneficial to each as they experience musth, whether they are in physical contact or not. Olfactory and acoustic communication are critical for mediating interaction among wild elephant groups (Vidya and Sukumar, 2005), and represent the primary forms of communication among bulls in zoos as they are rarely kept together (Hartley et al., 2019).

This study involved longitudinal assessments (9 to 22 years) of serum testosterone and cortisol concentrations in four male Asian elephants at a North American zoo with a long history of continuous hormone monitoring. The age range spanned 12 to 54 years, and 
each male exhibited regular annual musth. Three bulls developed and were treated for tuberculosis (TB) during the study. All four bulls died or were euthanized during the study. This extensive data set allowed us to examine variation in hormone concentrations in relation to musth, age, TB development and treatment, and health declines leading to death. We also examined adrenal response to social changes after a new bull was transferred in the zoo and after the deaths of male conspecifics.

Outcomes from this study can help us gain a better understanding of underlying conditions affecting patterns in testicular and adrenal GC activity, and how physiological or environmental changes may affect health and welfare of male Asian elephants.

\section{Materials and methods}

\section{$\underline{\text { Animals and sample collection }}$}

Subjects were adult male Asian elephants (Elephas maximus indicus) $(\mathrm{n}=4)$ housed at the AZA accredited Oregon Zoo. Elephants were both wild-born (n=2) and zoo-born $(n=2)$; with an age range of 12 to $\sim 54$ years during the study (Table 1$)$. This study was approved by the Animal Welfare Committee and the Research Review Committees of the Oregon Zoo (OZ). 
Table 1: Male elephants included in this study.

Individual, origin, age range during study, whether the individual exhibited normal musth cycles, number of samples analyzed, and statistical analyses performed for each individual.

\begin{tabular}{|c|c|c|c|l|}
\hline Elephant & Origin & Birth year & $\begin{array}{c}\text { Age range } \\
\text { (years) }\end{array}$ & \multicolumn{1}{|c|}{ Effects analyzed } \\
\hline Male1 & Wild & 1960 & $\sim 34-43$ & M, Age, DS \\
\hline Male2 & Zoo-born & 1962 & $32-54$ & M, Age, TM, DM, TBactive, TBtr, DS \\
\hline Male3 & Wild & 1971 & $\sim 34-44$ & M, Age, DM, TBactive, TBtr, DS \\
\hline Male4 & Zoo-born & 1983 & $12-32$ & R, M, Age, TM, DM, TBactive, TBtr, DS \\
\hline
\end{tabular}

$\mathrm{R}=$ Reproductive state

$\mathrm{M}=$ Musth hormone concentrations (see Tables 6 and 9)

Age $=$ Age analysis (see Tables 8 and 11)

$\mathrm{TM}=$ Transfer in of another adult male (see Table 12)

DM\# = Death of another adult male (Table 12)

TBactive $=$ Conversion to active $\mathrm{TB}$ (see Table 13)

TBtr $=$ anti-TB therapy (see Table 13)

DS = Health decline leading to death or euthanasia (see Table 14)

At any given time during the study period, two to three adult males were rotated among two outdoor yards and five indoor rooms, with olfactory and auditory contact, but limited physical and visual contact with each other. All bulls in this study had occasional physical contact with females for socialization and/or breeding. The three oldest bulls in this study sired multiple offspring. All bulls were given access to females regardless of whether they were in musth, but if any elephant indicated through behavioral signs that they preferred to be separated, the keepers opened access doors to allow them to do so. Male1 was given access to females only for breeding. Male2 was given access to the female herd for breeding and socialization, but unless a female was in estrous, he would choose to separate from the females. Male3 was well integrated with the females and the male offspring, and regularly spent time with them for socialization as well as for breeding. Male4 spent time with a compatible female, but not with the entire group. All 
bulls were managed in a protected contact system, where keepers and animals did not share the same space.

Social hierarchy among the bulls at any given time was determined by observations of behavioral posturing (bull assuming a dominant, neutral, or subordinate posture) and the physical location in adjacent yards in reaction to visual contact with another bull (Mike Keele and Bob Lee, pers comms). Over the course of the study there were two triads and two dyads of adult bulls: Male1-Male2-Male4 (1994 - 2003), Male2-Male4 (2003 2005), Male2-Male3-Male4 (2005 - 2015), and Male2-Male3 (2015). Male4 was subordinate in all groups. Male1 ranked higher than Male2, but later in the study Male2 imposed dominance and was perceived as higher in rank. Rank was not clear between Male2 and Male3 - both were large, dominant bulls - however, Male2 was aging, and they were not given access to each other to assess dominance. As is typical in male elephants, any non-musth bull deferred dominance to a musth bull.

Blood was collected (3-9 mL) into red top serum separator tubes from an ear or leg vein by elephant care staff (without sedation) as part of normal management. Blood was maintained at $\sim 4{ }^{\circ} \mathrm{C}$, and then centrifuged at $1500 \mathrm{~g}$ within a few hours to separate serum. Serum samples were stored at $\sim 20^{\circ} \mathrm{C}$ or colder until analysis. Samples were collected weekly, and in the morning to control for diurnal patterns of hormone secretion (Brown et al., 2010). Serum testosterone was analyzed weekly for routine management starting in 2006. Prior to 2006, serum testosterone was measured as part of previous research projects (Rasmussen et al., 1984; Rasmussen et al., 1990; Rasmussen and Perrin, 1999; 
Scott, 2002; Brown et al., 2007). Serum cortisol was analyzed in all previously collected samples in 2017.

\section{$\underline{\text { Hormone analysis }}$}

Testosterone concentrations in unextracted serum were initially measured using a solidphase ${ }^{125}$ I testosterone radioimmunoassay (RIA) (Siemens Healthcare Diagnostics Inc.) following the methods of Brown et al. (2007). Samples above high standard (below 20\% binding) were diluted in the zero calibrator (or standard) and re-analyzed. Assay sensitivity was $0.10 \mathrm{ng} / \mathrm{ml}$. The RIA antibody cross-reacted with testosterone (100\%), 19nortestosterone (22\%), 4-estren-17-ol-3-one (20\%), 11-ketotestosterone (16\%), $5 \alpha$-dihydrotestosterone (3.4\%), 19- hydroxandrostenedione (2.0\%), methyltestosterone (1.7\%), $11 \beta$-hydroxytestosterone (1.2\%), 4-estren-7 $\alpha$-methyl-17 $\beta$-ol-3-one (1.1\%), ethisterone (0.7\%), androstenedione ( $0.5 \%), 5 \beta$-androstan-3 $\alpha, 17 \beta$-diol $(0.4 \%)$,

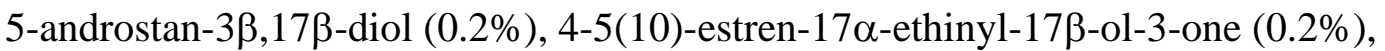
triamicinolone $(0.2 \%)$, norethindrone $(0.1 \%)$, and 15 other steroids at less than $0.1 \%$.

Production of this RIA was discontinued at the end of 2014, after which serum testosterone was quantified using a double-antibody testosterone enzyme immunoassay (EIA) with an anti-testosterone polyclonal primary antibody (R156/7; C. J. Munro, University of California, Davis, CA) and testosterone-horseradish peroxidase (HRP) tracer (C. Munro, UC Davis, CA). For plate coating, a secondary goat anti-rabbit IgG antibody (Cat. No. A009, Arbor Assays, Ann Arbor, MI) was diluted (10 $\mu \mathrm{g} / \mathrm{ml})$ in coating buffer (Cat. No. X108, 20X, Arbor Assays, Ann Arbor, MI) and added to each 
well $(150 \mu \mathrm{l})$ of a 96-well microtiter plate (Costar 9018, Corning Life Sciences, Tewkesbury, MA) followed by incubation at room temperature (RT) for 15-24 hours. The contents of the wells were emptied and the plates blotted dry to remove unbound antibody, then blocking solution (Cat. No. X109, 10X, Arbor Assays, Ann Arbor, MI) was added to each well $(250 \mu \mathrm{l})$ and incubated for 4-24 hours at RT. The contents of the wells were then emptied again, and plates were dried at RT in a desiccator cabinet. After drying (humidity <20\%), plates were vacuum-sealed in a foil bag with a 1-gram desiccant packet and stored at $4^{\circ} \mathrm{C}$ until use.

The R156/7 antibody $(1: 187,500)$ and HRP $(1: 100,000)$ were diluted in assay buffer (Cat. No. X065, 5X, Arbor Assays, Ann Arbor, MI). On the day of analysis, a pre-coated goat anti-rabbit IgG plate was equilibrated to room temperature ( $30 \mathrm{~min})$. Unextracted serum samples were incubated with equal quantities of dissociation reagent (X017, Arbor Assays) for 5 minutes and then diluted in assay buffer (1:20 to 1:100) and tested immediately. Testosterone standards $(0.023-6.0$ ng/ml; Steraloids, Inc., Newport, Rhode Island), internal controls and samples (50 $\mu 1$ each) were added to each well in duplicate, followed by $25 \mu \mathrm{lHRP}$, then $25 \mu \mathrm{l}$ of primary antibody to all wells except non-specific binding wells. The loaded plate was sealed and incubated at RT with shaking (500 rpm) for 2 hours. Unbound components were removed by washing 3 times with wash solution (Cat. No. X007, 20X, Arbor Assays, Ann Arbor, MI) and blotted dry, followed immediately by adding $100 \mu \mathrm{l}$ of a chromogen solution containing high kinetic tetramethylbenzidine (TMB) (2.5 mmol/L, Prod. No. TMB-HK, Moss, Inc., Pasadena, MD) to each well. The plate was sealed again and incubated at RT without shaking for 30 
min, then the reaction was halted by adding stop solution ( $50 \mu \mathrm{l} ; 1 \mathrm{~N}$ HCL, Ricca Chemical). Optical densities were determined by a Dynex plate reader at $450 \mathrm{~nm}$ with a reference of $630 \mathrm{~nm}$. Samples below 20\% binding were re-analyzed at a higher dilution. Assay sensitivity (based on $90 \%$ binding) was $0.015 \mathrm{ng} / \mathrm{ml}$. The R156/7 antibody is reported to cross-react with testosterone (100\%), 5 $\alpha$-dihydrotestosterone (57.37\%), androstenedione ( $0.27 \%)$ and less than $0.10 \%$ for 17 other steroids tested (C. Munro).

Cortisol concentrations in unextracted serum samples were measured using a solid-phase cortisol ${ }^{125}$ I radioimmunoassay (RIA) (CortiCote, MP Biomedicals, Santa Ana, CA; catalog \# 06B256440) following the methods of Brown et al. (2007). Assay sensitivity for the cortisol RIA was $2.5 \mathrm{ng} / \mathrm{ml}$. Cross reactivities for the cortisol RIA antibody were as follows: cortisol 100.0\%, prednisolone $94.1 \%$, 11-deoxycortisol $2.2 \%$, prednisone $1.2 \%$, corticosterone $1.2 \%$, cortisone $0.8 \%$, dexamethasone $0.8 \%, 17$ hydroxyprogesterone $<0.05 \%$, and metyrapone $<0.01 \%$.

The RIAs had been validated for elephants by demonstrating: (1) parallelism between dilutions of pooled serum samples to the respective standard curve preparation, and (2) significant (> 90\%) recovery of exogenous standard hormone added to pooled samples before analysis, and also through relevant biological tests (Brown et al., 1993; Brown et al., 2007). The testosterone EIA was validated by demonstrating parallelism and $>90 \%$ recovery and with biological tests of significant increases observed during musth. Values using the EIA and RIA assay for the same samples were strongly correlated, $r(49)=0.93$, although measured testosterone concentrations using the EIA were on average 1.8 times 
higher than the RIA. Thus, EIA results were converted for inclusion in this longitudinal dataset. The inter- and intra-assay coefficients of variation (CVs) were maintained below $15 \%$ and $10 \%$, respectively, by re-analyzing samples or plates that fell outside these criteria.

Determination of musth state

Records of musth signs

Physical and behavioral signs of musth were recorded by elephant care staff in daily keeper notes. Temporal gland secretion (TGS), urine dribbling (UD), and behavior changes were recorded using numerical scales defined by Scott (2002). Table 2 shows the behavior changes that were typically associated with musth in this group of bulls. The Oregon Zoo collaborated on the development of a more detailed musth scale for the physical or visible signs of TGS (Table 3) and UD (Table 4), and numerical record values were converted to this new scale for analysis (i.e., TGS $=1$ changed to 2 ; TGS=2 changed to 3$)$.

Table 2: Musth behavior scale for Oregon Zoo elephants

\begin{tabular}{|l|l|}
\hline 0 & No aggression, moves readily \\
\hline 1 & Somewhat "spacey" or uncooperative \\
\hline 2 & Reluctant to move, bangs on doors occasionally \\
\hline 3 & Considerable hesitation moving, bangs on doors often \\
\hline 4 & Very aggressive, refuses to move \\
\hline
\end{tabular}


Asian elephant musth scale of visible signs

This musth scale (Tables 3 and 4) was developed by Sharon Glaeser (Oregon Zoo), Shawn Finnell (Oregon Zoo), and Dr. Chaleamchat Somgird (Chiang Mai University, Thailand) and adapted from previous work by Jainudeen et al. (1972a), Poole (1987), Scott (2002), and Somgird et al. (2016). This scale has been approved by the Oregon Zoo, Chiang Mai University Faculty of Veterinary Medicine, Elephant Managers Association, Sabah Wildlife Department Wildlife Rescue Unit, and the International Union for Conservation and Nature (IUCN) Asian Elephant Specialist Group. 
Table 3: Temporal Gland Swelling and Secretion (TGS)

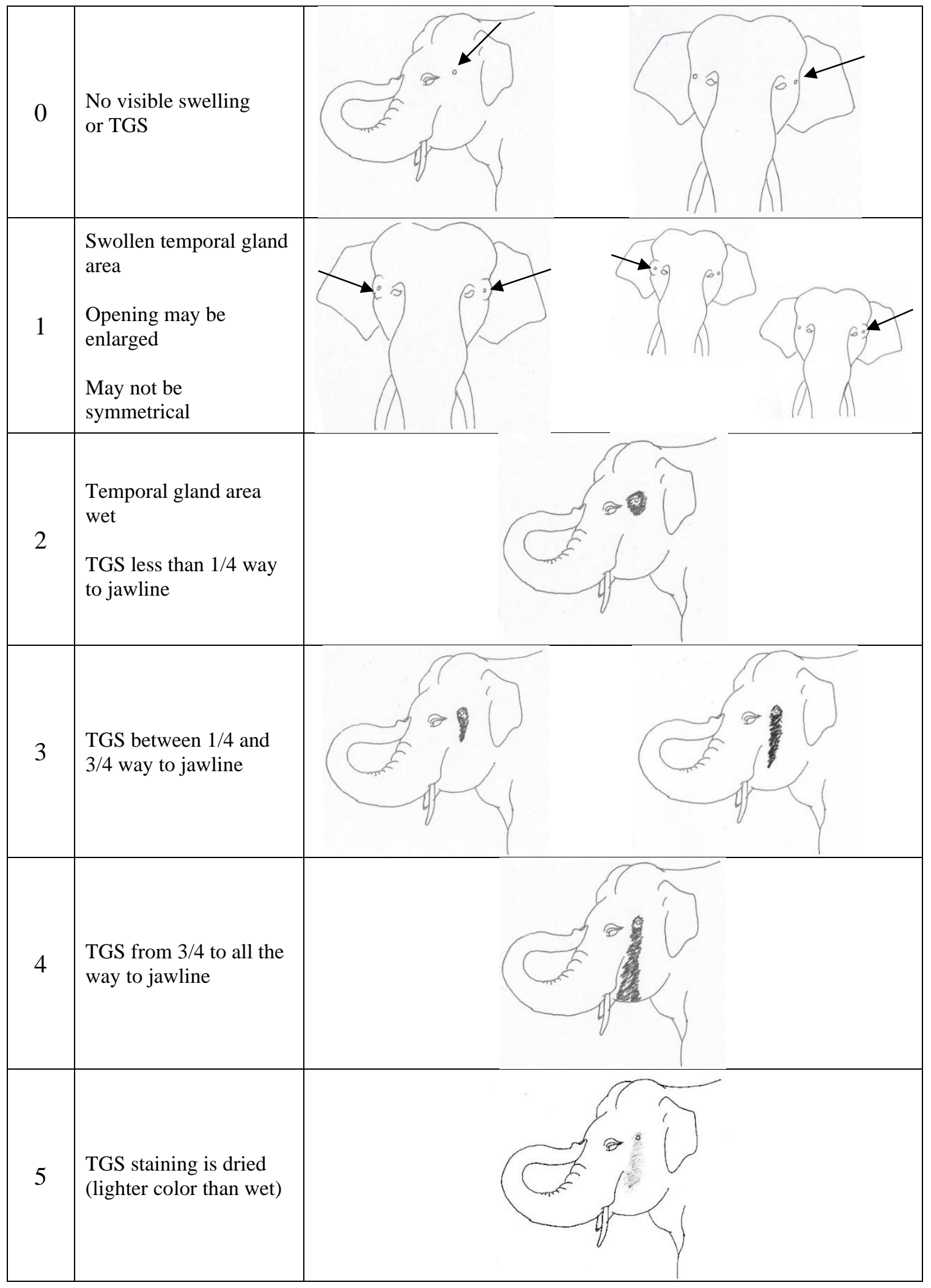


Table 4: Urine Dribbling (UD)

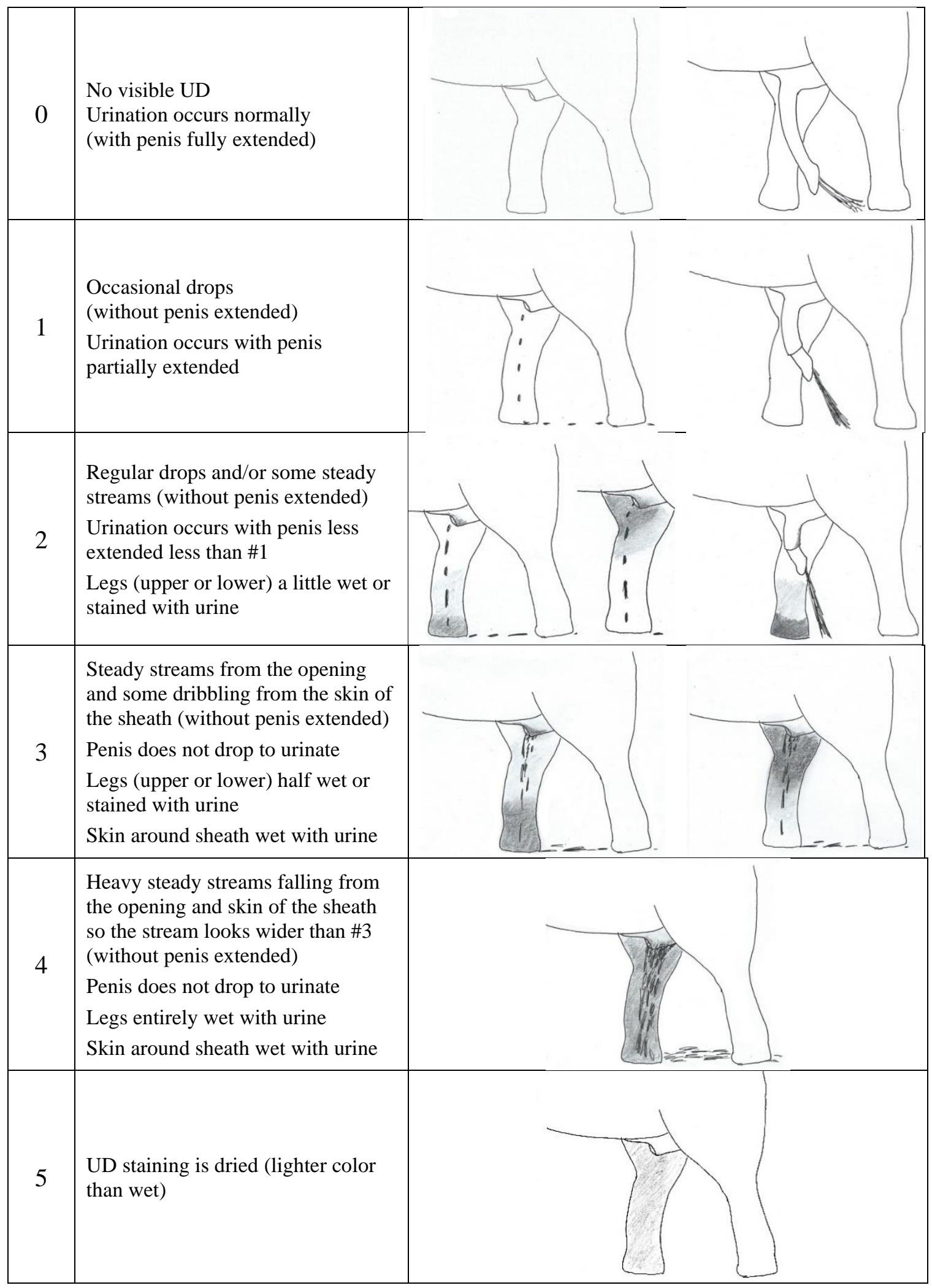




\section{Criteria for defining musth}

Stages of musth were defined by physical and behavioral signs and associated increased serum testosterone (Table 5). The minimum testosterone concentration for determining musth state was based on previous studies by Scott (2002), Brown et al. (2007), and Chave et al. (2019).

Table 5: Criteria used to define musth in this study

\begin{tabular}{|c|c|c|c|c|c|}
\hline \multirow{2}{*}{$\begin{array}{c}\text { Musth } \\
\text { Status }\end{array}$} & \multicolumn{2}{|c|}{ Serum Testosterone } & TGS $^{\mathrm{a}}$ & UD $^{\mathrm{b}}$ & Behavior $^{\mathbf{c}}$ \\
\cline { 2 - 6 } & Concentration & Duration & & \\
\hline $\begin{array}{c}\text { Inter-musth } / \\
\text { Non-musth }\end{array}$ & $<10 \mathrm{ng} / \mathrm{ml}$ & $\geq 2$ weeks & $0^{\mathrm{d}}$ & 0 & $0^{\mathrm{d}}$ \\
\hline Pre-musth & $\geq 10 \mathrm{ng} / \mathrm{ml}$ & no criteria & 1 & 0 & $1-4$ \\
\hline Musth & $\geq 10 \mathrm{ng} / \mathrm{ml}$ & $\begin{array}{c}\geq 4 \text { weeks } \\
\text { (including } \\
\text { pre/post musth) }\end{array}$ & $2-4$ & $1-4$ & $1-4$ \\
\hline Post-musth & $\geq 10 \mathrm{ng} / \mathrm{ml}$ & no criteria & $0^{\mathrm{e}}$ & $0^{\mathrm{e}}$ & $1-4$ \\
\hline
\end{tabular}

${ }^{a}$ Numerical values defined in Table 3

${ }^{\mathrm{b}}$ Numerical values defined in Table 4

${ }^{\mathrm{c}}$ Numerical values defined in Table 2

${ }^{\mathrm{d}}$ Behaviors typical of musth and mild TGS were sometimes observed during inter-musth periods coincident with musth in other bulls, estrous in females, or breeding activity with females.

${ }^{\mathrm{e}}$ TGS/UD of 0 (versus 5) was recorded to indicates cessation of TGS and UD.

The pre- and post-musth stages were included in the full musth state for analysis since musth occurs on a spectrum, and physiological changes (i.e., increased testosterone and volatile secretions) also occur in these transitional stages (Jainudeen et al., 1972a;

Rasmussen et al., 1990; Rasmussen and Perrin, 1999; Schulte and Rasmussen, 1999; Somgird et al., 2016). Furthermore, in some years the historical records indicated only that an animal was in "musth", and the details of TGS, UD, and behavior were not included to help delineate pre- and post-musth from full musth. 


\section{Calculation of individual musth thresholds}

After musth periods were defined using the criteria in Table 5, the testosterone concentrations corresponding to the beginning and end of musth (for full musth) were used to calculate actual minimum, median, and mean thresholds concentrations for each individual. To aid in future research, these thresholds were then compared to thresholds calculated using methods by Chave et al. (2019) to define musth based solely on testosterone concentrations.

\section{Demographics and life events}

Origin of birth, birth date, and life event data of births, deaths, and facility transfers (change in physical location) for each bull were obtained from the AZA Asian Elephant Regional Studbook (Lee, 2019). During the study, one adult male was transferred in to Oregon Zoo and all four died during the course of the study, which constituted social life events (i.e., events involving herdmates), and events that each individual physically experienced (i.e., their own death).

\section{$\underline{\text { Tuberculosis diagnosis and treatment }}$}

Three bulls in this study were diagnosed with Mycobacterium tuberculosis (M. $t b$ ), a primary causative agent of TB. The outbreak diagnosis and treatment are documented in detail by Miller et al. (2018). The first case was detected by isolation of $M$. tb during routine trunk wash (TW) culture testing, Retrospective antibody analyses revealed seroconversion one year prior to diagnosis. Serological testing of all elephants identified two additional bulls with detectable antibodies, but which remained culture-negative, 
then in the following months, $M$. $t b$ was isolated from TW samples of those two bulls. No clinical signs attributable to TB were detected in any elephant. All infected elephants received anti-TB therapy as described by Miller et al. (2018) (elephant A=Male4; $\mathrm{B}=$ Male2, $\mathrm{C}=$ Male3). Health records were reviewed to identify dates of the last negative and first positive TW to identify the period of transition to active shedding of $M . t b$. (Male4: 14 months; Male2: 10 weeks; Male3: 9 weeks) and also anti-TB therapy start and end dates.

\section{$\underline{\text { Health declines }}$}

All four bulls died or were euthanized during the study. Male1 died of sepsis; no euthanasia was performed on him. Two bulls that were treated for TB were eventually euthanized for reasons unrelated to $M$. $t b$. Male4 was euthanized due to chronic progressive arthritis in multiple joints, and had been on antimycobacterial treatment for 20 months before and up to death. Male3 was euthanized for severe extensive chronic osteoarthritis, and had been on antimycobacterial treatment for 15 months before and at the time of death. Male2 was euthanized due to development of a drug-resistant strain of M. $t b$; his treatment was discontinued after confirmation of drug resistance 4 months prior euthanasia.

\section{$\underline{\text { Data analysis }}$}

Median, range, mean, standard deviation (SD), and coefficient of variation $(\mathrm{CV})$ in serum testosterone and cortisol concentrations were calculated for each individual and all elephants combined, for the entire study period, during musth and inter-musth, and in 
Male4 including the transition from a pre-pubertal to adolescent state and a first musth cycle. All descriptive statistics were calculated using Excel (Microsoft ${ }^{\circ}$ Office Excel 2016; Microsoft, Corp., Redmond, WA, USA). Coefficients of variation (CV) in cortisol concentration were calculated for states of musth and inter-musth. Differences between CVs were determined by the Brown-Forsythe Test (Brown and Forsythe, 1974; Dayton et al., 2016), run in R version 3.6.1 (R Core Team, 2020) using the package "onewaytests" (Dag et al., 2018). Significance was assessed at the 0.05 level for all analyses.

Hormone data were analyzed using generalized linear mixed models (GLMMs) in MLwiN version 2.02 (Rasbash et al., 2005) to investigate changes in mean testosterone concentrations in association development and aging during musth and inter-musth periods; and changes in mean cortisol concentrations according to reproductive state (prepubertal, adolescent), musth state (musth, inter-musth), age, origin of birth, period of transition to active shedding of $M . t b$. start/end of anti-TB therapy, health decline leading to death or euthanasia, and in response to social life events (transfers and deaths of other bulls). GLMMs allow random effects to be incorporated into the model (Bolker et al., 2009; Harrison et al., 2018) to control for non-independence of data, which in this study involved repeated serum samples per subject. Kolmogorov-Smirnov normality tests and examination of plots showed distributions of hormone data were non-normal, so hormone data were $\log 10$ transformed to improve the distribution for the GLMMs.

Separate models were created for each elephant to investigate the effect of physiological and social changes on an individual-level, and with all elephants combined to investigate 
group-level effects of age and origin of birth. GLMMs in this study were based on models by Edwards et al. (2015) and (2016), and Glaeser et al. (in review, Chapter 2) The dependent variable (hormone data), random effects (date of sample collection), and fixed effects were incorporated into each model. Reproductive stage (prepubertal, adolescent), musth state, transition to active shedding of $M$. tb, anti-TB therapy, social life events, health decline and 10-year age categories were fitted individually as categorical fixed effects; age was fitted as a continuous fixed effect.

Event-based effects (social life events, TB transition, anti-TB therapy, and health decline leading to death or euthanasia) were defined with time periods surrounding the event. Social life events (transfer and death of other males) were classified as pre- and postevent, with an equal duration of 30 days before and 30 days after the event (total 60 days), and with the timeframe extended to 60 days before and after the event to determine if the addition or removal of a bull would have an impact on adrenal GC activity over a longer time period. The period of transition to active shedding of $M$. $t b$. was defined as time between the last culture-negative TW to detection of active $M$. $t b$., which differed across individuals. Comparisons were made between this transition period and the preceding time period of equal duration, in 30-day blocks to locate any acute changes in adrenal GC activity associated with disease transition. The start and completion of antiTB therapy were classified with an equal duration of 30 days before and 30 days after therapy start or end (total 60 days). The period leading up to death by euthanasia $(n=3)$ and to natural death $(\mathrm{n}=1)$ were modelled with a timeframe of the final 30 days of life compared to the preceding 30 days (total 60 days). The day the event occurred was 
included in the post-event time period. The models were first run to determine differences in hormone concentration with musth state, then these effects were taken into account by inclusion as covariates in the event-based models.

A normal error structure was used for all models. The significance of each fixed effect compared to the reference category was determined using the Wald statistic and chi-

squared $\left(\chi^{2}\right)$ distribution, with alpha set to 0.05 ; and significance of pair-wise

comparisons was determined using $\chi^{2,}$ with alpha set to 0.05 . Predicted means and standard errors (SE) of $\log 10$ cortisol and $\log 10$ testosterone concentrations were back transformed to generate charts.

\section{Results}

\section{Longitudinal serum testosterone patterns}

Serum testosterone concentrations (median, range, and mean) and variability (SD and CV) overall and during musth and inter-musth periods are presented in Table 6. Mean hormone concentrations varied within and among individual bulls during both musth and inter-musth periods. 


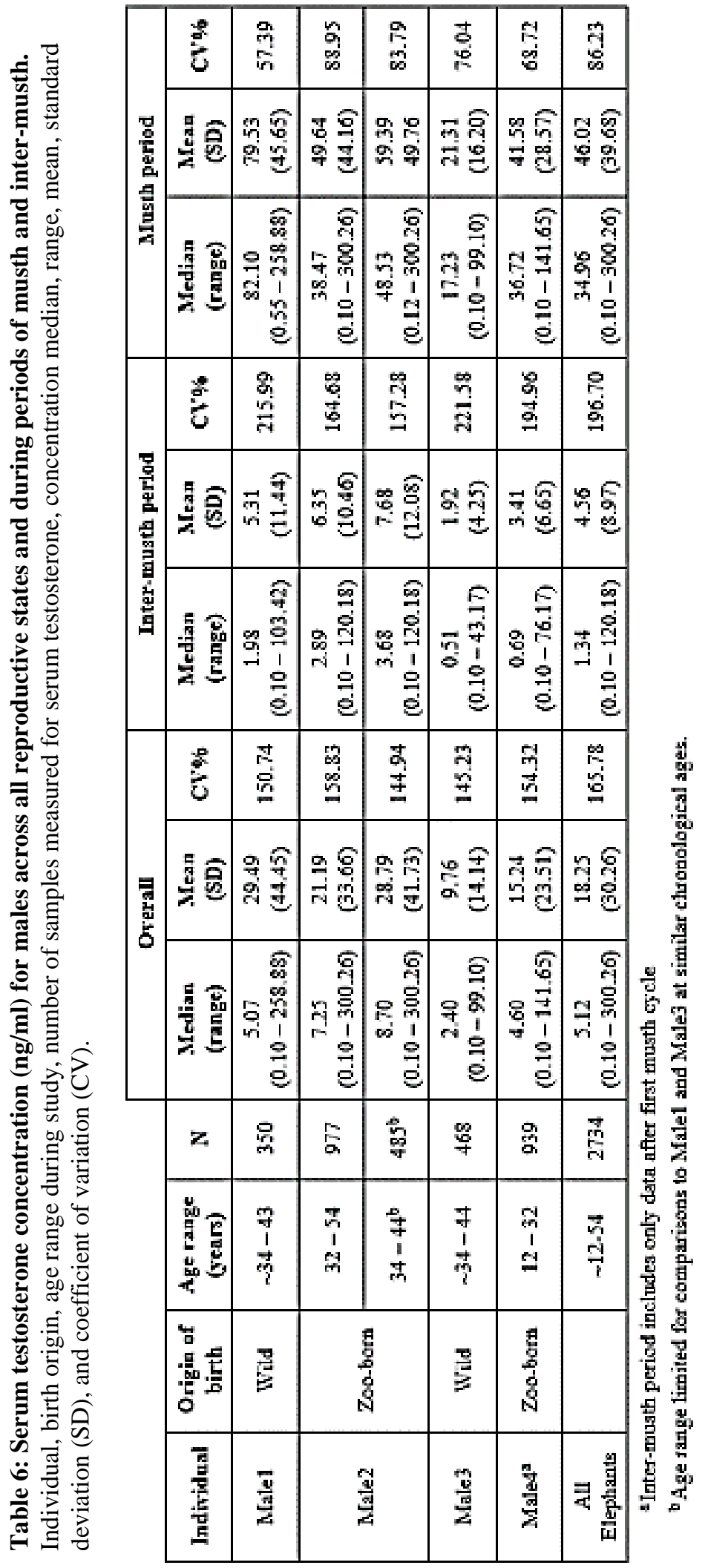


Musth

Musth was characterized by elevated serum testosterone measurements corresponding with varying duration and intensity of temporal gland secretion (TGS), urine dribbling (UD), and behavioral changes. In general, behavior changes were noted prior to TGS and UD, and TGS and UD occurred as testosterone began to increase or within a few weeks thereafter. All bulls exhibited regular annual musth cycles, which generally occurred between late winter to mid-summer in the two oldest bulls, followed by mid-summer to late fall in the youngest bull, then late summer to winter in the bull that transferred into the facility. There was no evidence of synchronization; however, in some years musth cycles overlapped more than others. There were no extended periods of elevated testosterone that were not associated with recorded behavioral and physical musth signs, with some exceptions. In the 1990s, the two oldest bulls sometimes exhibited a second shorter period of elevated testosterone after coming out of musth, with keeper records stating that an immediate return to full daily diets caused them to go back into musth. Feeding regimes were changed to increase hay and grain slowly in the weeks after musth, and by 2001 a recurrence of musth was rare. Male2 and Male4 exhibited testosterone elevations when other bulls were in musth, sometimes coincident with 1-2 weeks of TGS and UD. Male3 sometimes showed elevations in testosterone and single days of TGS/UD surrounding ovulation in three females, and/or when he had access to the females for breeding or socialization. In Male2, peak testosterone concentrations during musth declined from 2007 through 2017 with age. His musth episodes from 2014 through end of life, which coincided with anti-TB therapy, were described by elephant care staff as "erratic", and were characterized by a less intense annual musth, additional shorter musth 
periods, and a greater percentage of time with increased testosterone concentrations (Fig. 1A) and expression of musth-like behaviors. Male1 exhibited annual musth over a 3month range of starting dates, and in some years exhibited musth in two distinct time periods within the time frame that in previous or subsequent years had only one musth event (Fig. 1B).

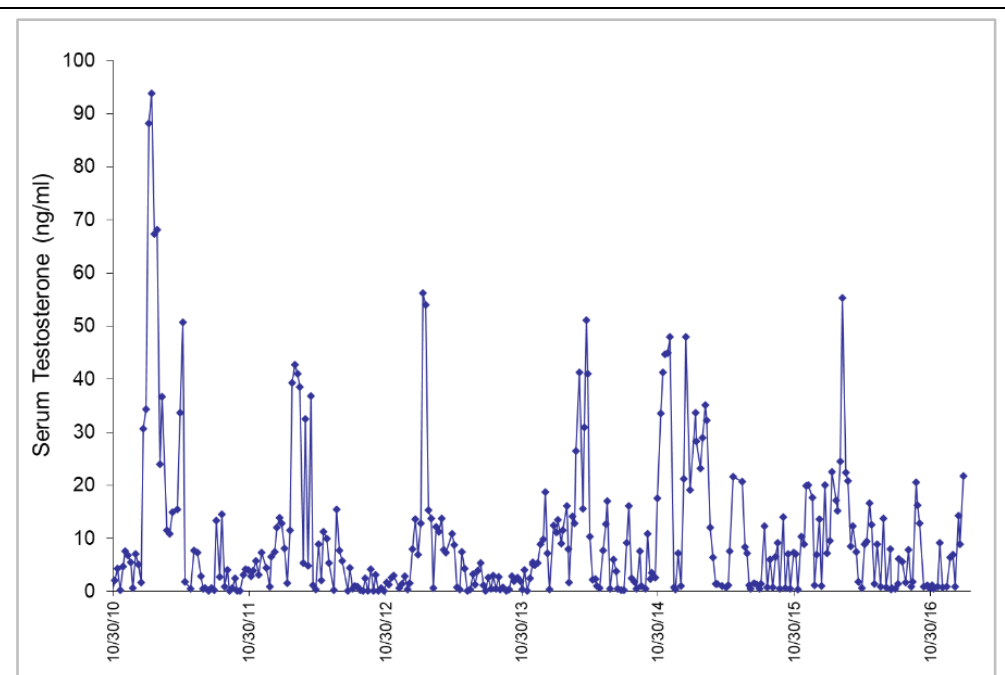

(A)

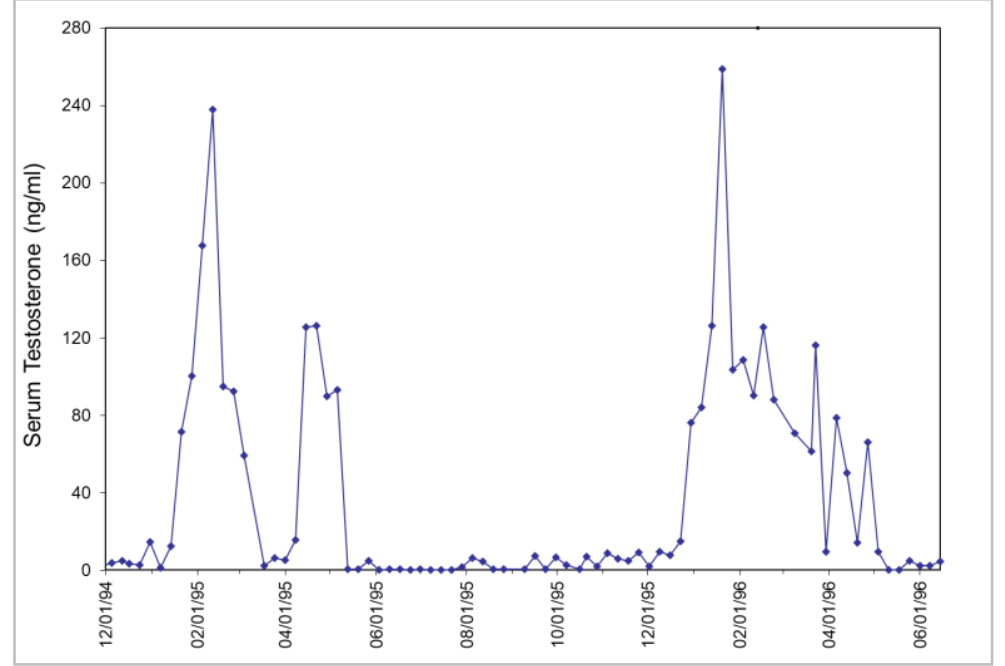

(B)

Fig 1: Patterns of testosterone during musth.

(A) Male2: Profile shows change in testosterone from 2014 to end of life. (B) Male1: Profile shows musth exhibited in two distinct time periods. 


\section{Musth threshold testosterone concentrations}

Testosterone concentrations corresponding to the beginning and end of musth for each individual are shown in Table 7, as well as the calculated minimum using methods developed by Chave et al. (2019) for comparison.

Table 7: Serum testosterone concentrations (ng/ml) corresponding to the beginning and end of musth

\begin{tabular}{|c|c|c|c|c|}
\hline \multirow{2}{*}{ Individual } & \multicolumn{3}{|c|}{ Threshold values } & \multirow{2}{*}{$\begin{array}{l}\text { Minimum for first musth point using } \\
\text { methods of Chave et al., } 2019\end{array}$} \\
\hline & Minimum & Median & Mean & \\
\hline Male1 & 16 & 69 & 72 & 67 \\
\hline Male2 & 11 & 43 & 49 & 51 \\
\hline Male3 & 13 & 16 & 18 & 21 \\
\hline Male4 & 10 & 40 & 39 & 35 \\
\hline
\end{tabular}

\section{Effects of age and dominance status on testosterone across bulls}

There was no definitive relation of higher mean (Table 6) or threshold (Table 7) testosterone concentrations to age or dominance in this study. The older and dominant bulls in the Male1-Male2-Male4 triad had the highest testosterone concentrations, and concentrations for Male2 remained higher than Male4 throughout this study; however, this dominance-related pattern was not exhibited in the Male2-Male3-Male4 triad.

Furthermore, this longitudinal dataset allowed comparisons across dominant bulls at similar chronological ages (Male1, ages $\sim 34-43$; Male2 and Male3, age $~ 34-44$ ), and confirmed an almost four-fold range in mean testosterone concentrations across bulls in the same age group and dominance rank. 


\section{Effects of age on testosterone within individuals}

Age was a significant predictor of serum testosterone in all bulls, with testosterone decreasing with age overall (Table 8) in mature bulls. In two bulls, GLMMs predicted testosterone was significantly lower in the older age categories as well, and in the other two bulls the difference across age categories was not significant. However, all bulls showed decreasing testosterone based on visual inspection of hormone profiles and decreasing calculated mean concentrations across age categories (Figs. 1 and 2). The youngest male (Male4: Figs. 2E, F) experienced his first musth cycle at 15 years of age, and showed increased testosterone with age as he entered puberty and matured during his early musth cycles until around age 20 , then exhibited decreasing testosterone. The three older males all showed decreased testosterone after 31-40 years of age. Age-related decreases occurred prior to detection and treatment of $M t b$ in all individuals.

Fig. 3 shows how GLMM predicted means of $\log 10$ transformed testosterone data were closer to calculated median than mean values due to the data being extremely rightskewed. 
Table 8: Age as a predictor of testosterone.

Individual, age range of analysis, age variable, effect size with standard error (SE), Wald statistic, and pvalue from GLMMs; and relative effect of age on mean testosterone concentrations. Degrees of freedom (df) is 1 in all pair-wise comparisons.

\begin{tabular}{|c|c|c|c|c|c|c|}
\hline Individual & $\begin{array}{l}\text { Age Range } \\
\text { of Analysis }\end{array}$ & Age Variable & Effect Size (SE) & Wald & $\mathbf{P}$ & $\begin{array}{c}\text { Age } \\
\text { Effect }\end{array}$ \\
\hline \multirow[t]{3}{*}{ Male1 } & \multirow[t]{3}{*}{$\sim 34-43$} & Age (years) & $-0.066(0.021)$ & 9.681 & 0.002 & Decreases \\
\hline & & $31-40$ (reference) & - & - & - & \\
\hline & & $41-50$ & $-0.097(0.125)$ & 0.606 & 0.436 & -- \\
\hline \multirow[t]{4}{*}{ Male2 } & \multirow{4}{*}{$32-53$} & Age (years) & $-0.027(0.004)$ & 37.043 & $<0.001$ & Decreases \\
\hline & & $31-40$ (reference) & - & - & - & \\
\hline & & $41-50$ & $-0.289(0.056)$ & 26.262 & $<0.001$ & Lower \\
\hline & & $51-60$ & $-0.432(0.088)$ & 23.858 & $<0.001$ & Lower \\
\hline \multirow[t]{3}{*}{ Male3 } & \multirow{3}{*}{$\sim 34-44$} & Age (years) & $-0.090(0.015)$ & 38.079 & $<0.001$ & Decreases \\
\hline & & $31-40$ (reference) & - & - & - & \\
\hline & & $41-50$ & $-0.495(0.082)$ & 36.783 & $<0.001$ & Lower \\
\hline \multirow[t]{4}{*}{ Male $^{\mathrm{a}}$} & \multirow{4}{*}{$15-32$} & Age (years) & $-0.017(0.005)$ & 10.509 & 0.001 & Decreases \\
\hline & & $11-20$ (reference) & - & - & - & \\
\hline & & $21-30$ & $-0.081(0.063)$ & 1.655 & 0.198 & -- \\
\hline & & $31-40$ & $0.167(0.128)$ & 1.697 & 0.193 & -- \\
\hline
\end{tabular}

-- No significant difference or interaction is not significant

${ }^{a}$ Age analysis includes only mature bulls, which starts at age 15 for this individual. 

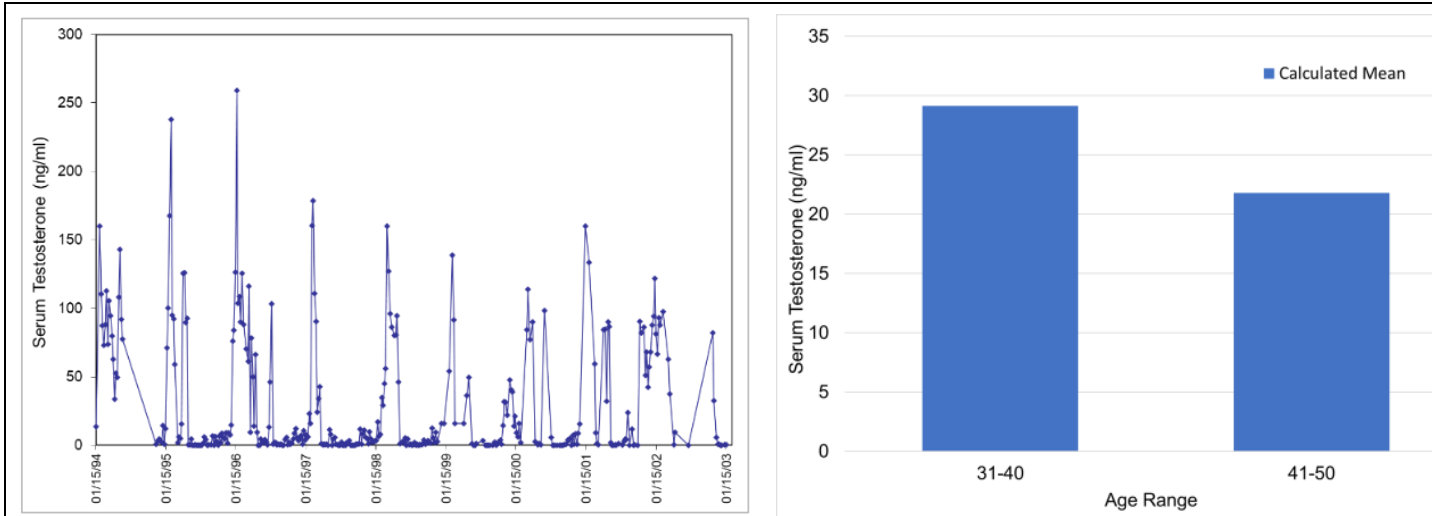

(A)

(B)
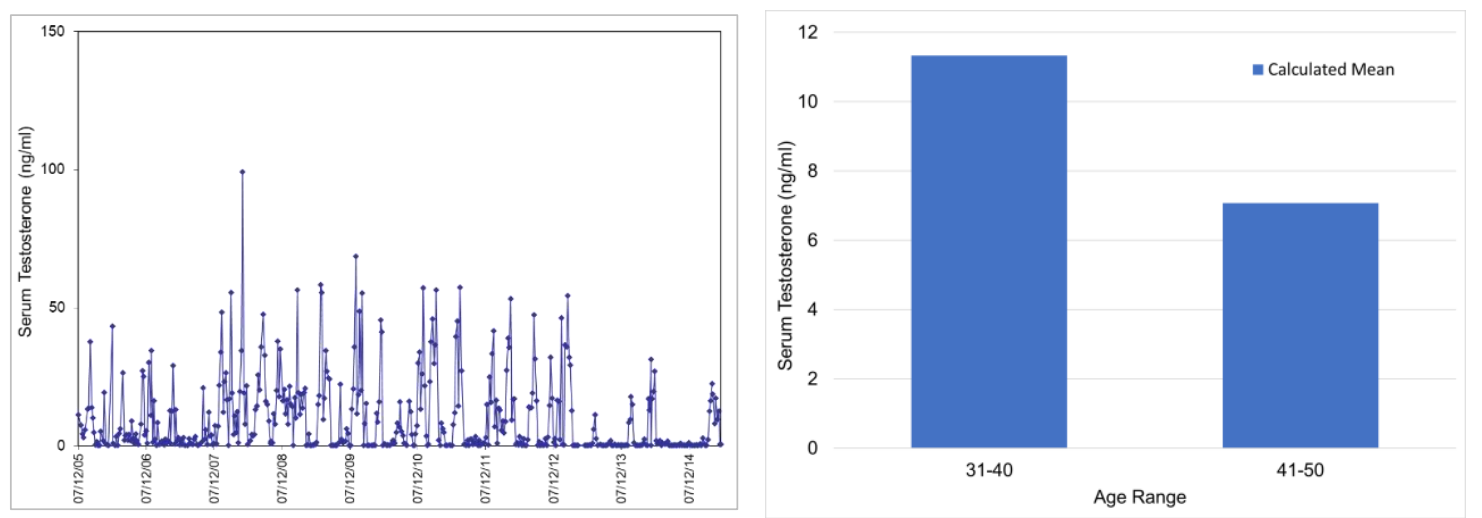

(C)

(D)
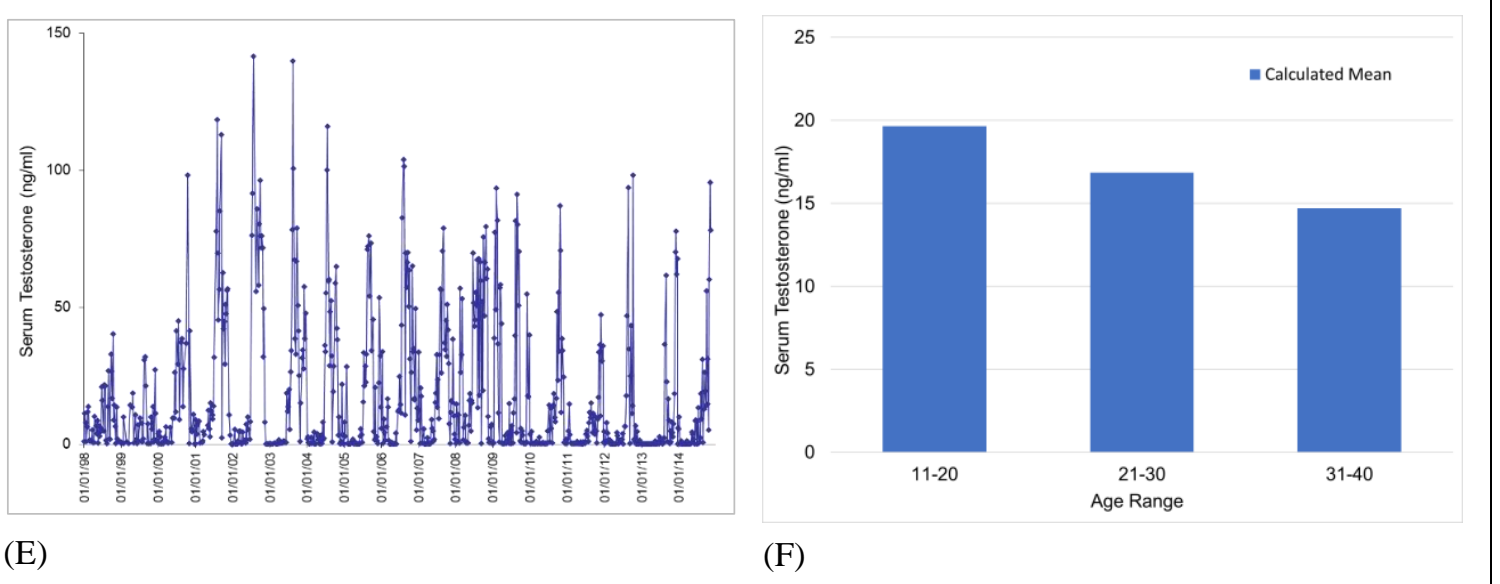

(E)

\section{(F)}

Fig 2: Age and changes in testosterone.

(A) Male1: Profile shows decreasing peak testosterone concentrations. (B) Male1: Comparison of calculated mean concentrations across age categories. (C) Male3: Profile shows decreasing peak testosterone concentrations. (D) Male3: Comparison of calculated mean concentrations across age categories. (E) Male4: Profile shows increasing peak testosterone concentrations from first musth at age 15 to musth at age 20 then peaks decrease. (F) Male4: Comparison of calculated mean concentrations across age categories. 

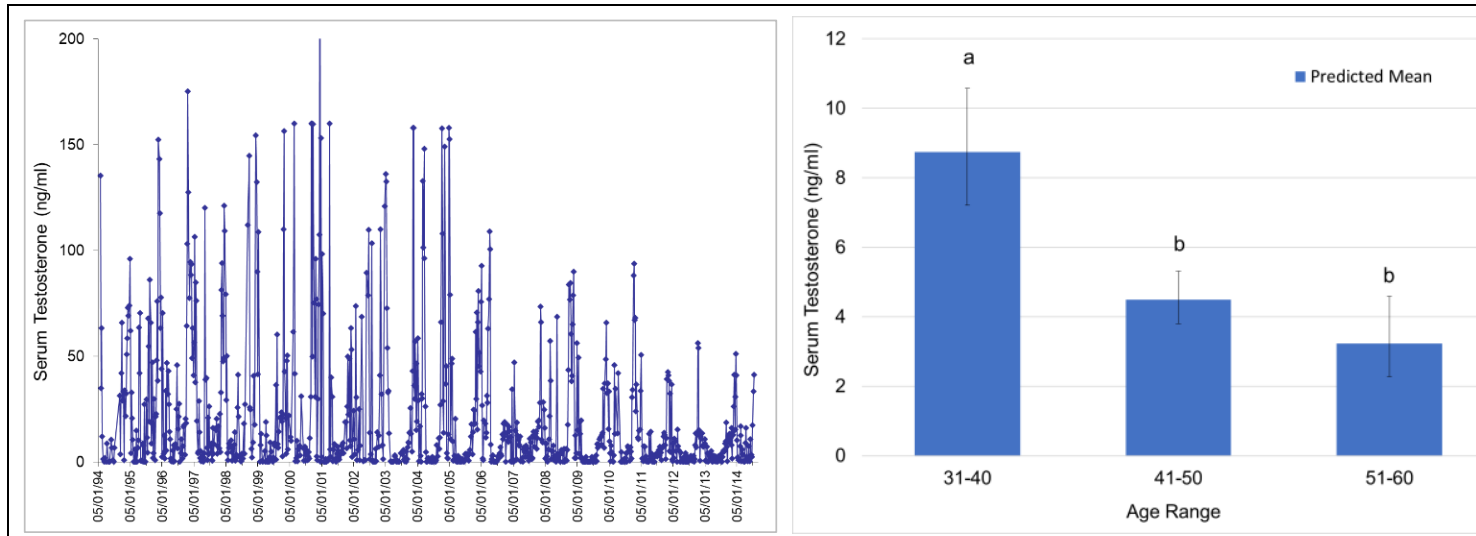

(A)

(B)
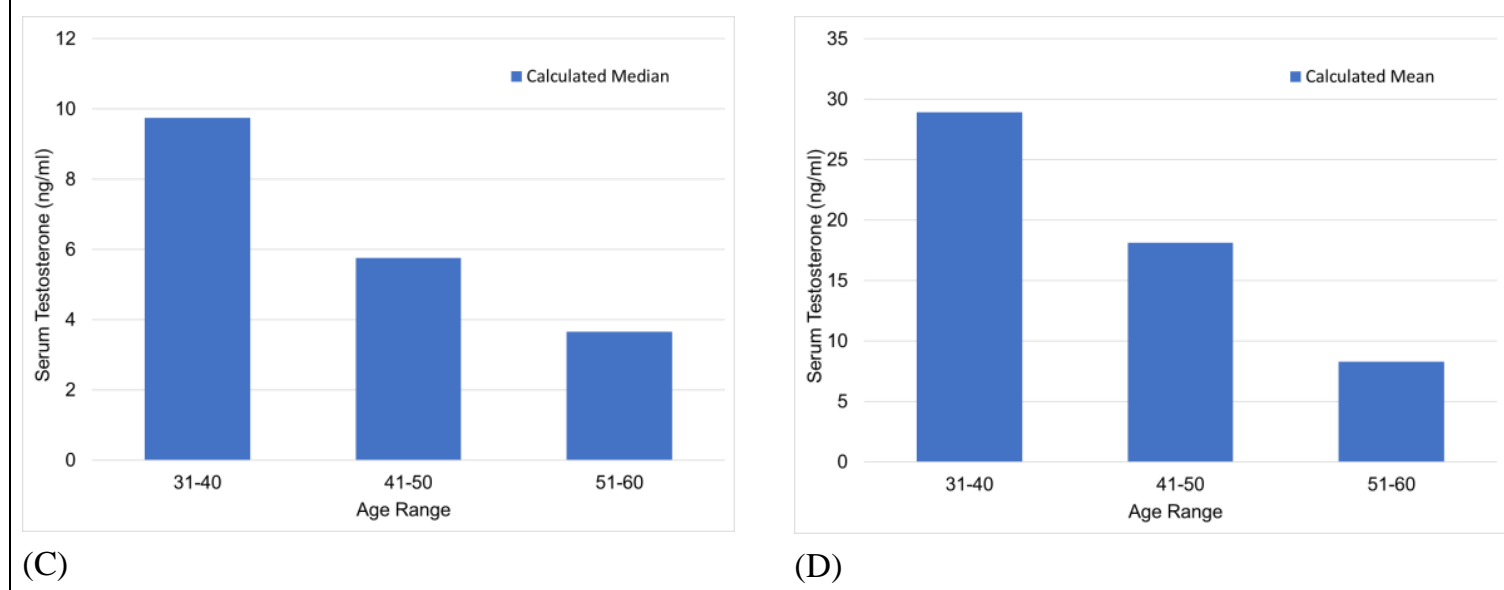

Fig 3: Age and changes in testosterone comparing GLMM predications and calculations.

(A) Male2: Profiles shows decreasing peak testosterone concentrations. (B) Male2: Prediction from GLMM for serum testosterone concentration comparing mean values across age categories (error bars represent standard error of the prediction). Letters denote significant differences in mean values. (C) Male2: Comparison of calculated median concentrations across age categories showing similar values to predicted means in an extremely right-skewed distribution. (D) Male2: Comparison of calculated mean concentrations across age categories.

\section{$\underline{\text { Longitudinal serum cortisol patterns }}$}

Serum cortisol concentrations (median, range, and mean) and variability (SD and CV)

overall and during musth and inter-musth periods are presented in Tables 9. Mean

hormone concentrations varied within and among individual bulls during both musth and inter-musth periods. 


\begin{tabular}{|c|c|c|c|c|c|c|c|c|}
\hline \multirow{3}{*}{ 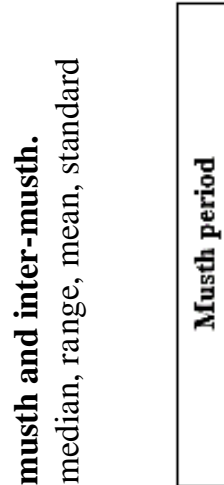 } & $\stackrel{8}{8}$ & 帒 & กิ่ & $\begin{array}{l}\text { 古 } \\
\text { 崫 }\end{array}$ & ถิ & $\begin{array}{l}\text { 岁 } \\
\text { 号 }\end{array}$ & 苔 & $\vec{J}$ \\
\hline & 鹿 & 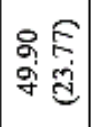 & $\begin{array}{ll}\infty & 6 \\
0 & 1 \\
0 & 0 \\
\end{array}$ & 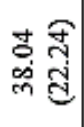 & 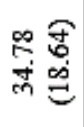 & 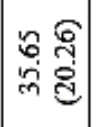 & 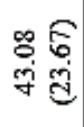 & 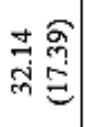 \\
\hline & 堅 & 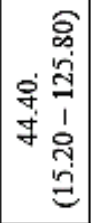 & 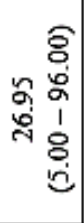 & 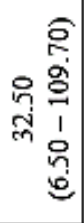 & 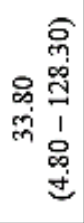 & 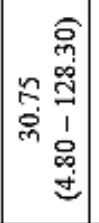 & 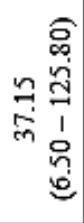 & 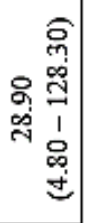 \\
\hline 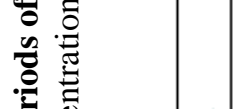 & : & 号 & $\begin{array}{l}8 \\
8 \\
10\end{array}$ & 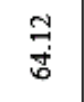 & ๓ุ & $\begin{array}{l}\text { 움 } \\
\text { 뭄 }\end{array}$ & $\overrightarrow{0}$ & 芦 \\
\hline 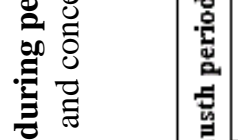 & 递完 & 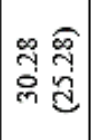 & 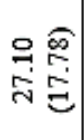 & 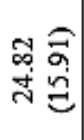 & $\begin{array}{l}\text { ㅇ: } \\
\text { 品 }\end{array}$ & 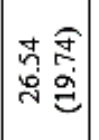 & 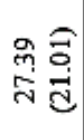 & 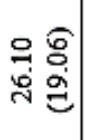 \\
\hline $\begin{array}{l}0 \\
0 \\
0 \\
0 \\
0 \\
0 \\
0\end{array}$ & 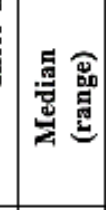 & 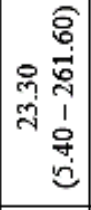 & 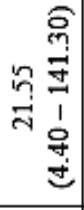 & 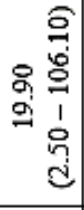 & 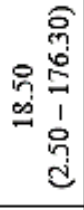 & 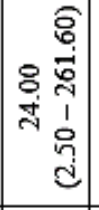 & 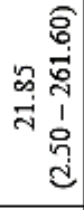 & 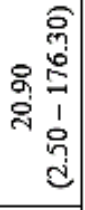 \\
\hline$\cong$ & : & तે & $\frac{8}{8}$ & $\begin{array}{l}\text { गे } \\
\text { है }\end{array}$ & $\stackrel{c}{a}$ & $\begin{array}{l}\text { ng } \\
\text { gi }\end{array}$ & 骂 & $\begin{array}{l}\text { g } \\
\text { ğ }\end{array}$ \\
\hline 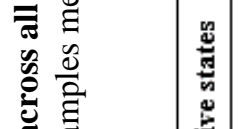 & 㓙 & 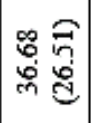 & 空 & 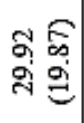 & 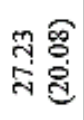 & 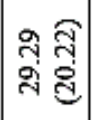 & 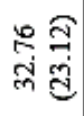 & 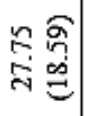 \\
\hline 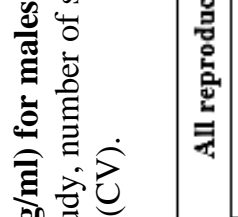 & 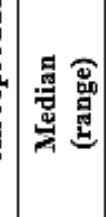 & 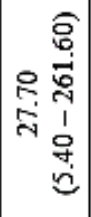 & 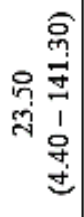 & 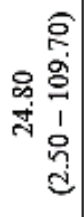 & 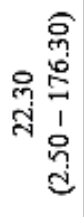 & 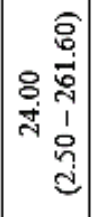 & 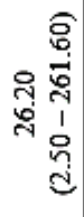 & 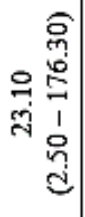 \\
\hline & '云 & 品 & 兽 & $\tilde{\precsim}$ & 茄 & 哭 & 气ू & ฒ̂̀ \\
\hline 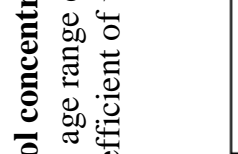 & $\begin{array}{l}\text { 品 } \\
\text { 量 } \\
\text { 总 } \\
\text { 品 }\end{array}$ & $\begin{array}{l}\text { 等 } \\
\text { 方 } \\
\text { ? }\end{array}$ & $\begin{array}{l}\vec{n} \\
1 \\
\text { c } \\
\text { ch }\end{array}$ & 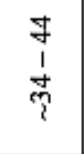 & $\begin{array}{l}\approx \\
\cdots \\
1 \\
\approx\end{array}$ & 究 & $\begin{array}{l}\text { J } \\
1 \\
\text { ț } \\
\text { ? }\end{array}$ & \begin{tabular}{l} 
\pm \\
1 \\
1 \\
\multirow{2}{*}{}
\end{tabular} \\
\hline 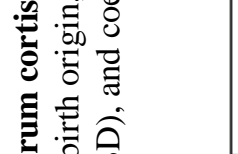 & 岁 & 胥 & 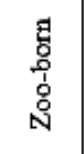 & 胥 & 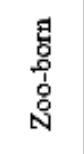 & & & \\
\hline.$\stackrel{0}{0}$. & 㬟 & 离 & 苋 & 证 & 蒁 & 层营 & 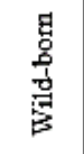 & 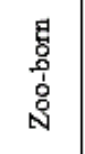 \\
\hline
\end{tabular}




\section{Effects of puberty, musth and testosterone on cortisol}

Cortisol was positively correlated with testosterone, and was higher in musth compared to inter-musth in all individuals and all bulls combined (Table 10). Sexual maturation was a significant predictor of mean cortisol concentration $($ GLMM coefficient $=0.131, \mathrm{SE}=$ 0.027, $\left.\chi^{2}=24.525, \mathrm{df}=1, \mathrm{p}=<0.001\right)$ in the bull that began exhibiting musth during the study (Male4), with cortisol being higher after sexual maturity compared to the prepubertal period, likely as a result of higher overall mean testosterone with annual musth and the positive correlation of cortisol and testosterone.

Table 10: Testosterone and musth as a predictor of adrenal GC activity.

Fixed effect, effect size with standard error (SE), Wald statistic, and p-value from GLMMs. Degrees of freedom (df) is 1 in all pair-wise comparisons.

\begin{tabular}{|l|l|c|c|c|}
\hline Fixed Effect & Individual(s) & Effect Size (SE) & Wald & P \\
\hline \multirow{4}{*}{ Testosterone } & Male1 & $0.129(0.014)$ & 85.048 & $<\mathbf{0 . 0 0 1}$ \\
\cline { 2 - 5 } & Male2 & $0.028(0.009)$ & 8.504 & $\mathbf{0 . 0 0 4}$ \\
\cline { 2 - 5 } & Male3 & $0.128(0.013)$ & 61.284 & $<\mathbf{0 . 0 0 1}$ \\
\cline { 2 - 5 } & Male4 & $0.070(0.011)$ & 38.353 & $<\mathbf{0 . 0 0 1}$ \\
\cline { 2 - 5 } & All elephants & $0.075(0.006)$ & 161.041 & $<\mathbf{0 . 0 0 1}$ \\
\hline Inter-musth* & - & - & - & - \\
\hline Musth & Male1 & $0.259(0.027)$ & 93.600 & $<\mathbf{0 . 0 0 1}$ \\
\hline & Male2 & $0.060(0.016)$ & 14.523 & $<\mathbf{0 . 0 0 1}$ \\
\hline & Male3 & $0.187(0.024)$ & 61.284 & $<\mathbf{0 . 0 0 1}$ \\
\hline & Male4 & $0.204(0.024)$ & 74.687 & $<\mathbf{0 . 0 0 1}$ \\
\hline & All elephants & $0.152(0.011)$ & 194.919 & $<\mathbf{0 . 0 0 1}$ \\
\hline
\end{tabular}

* denotes the reference category for GLMMs 


\section{Effects of age on cortisol}

There was an overall increase in mean serum cortisol with respect to age in all bulls combined. Across bulls, mean cortisol concentrations were lowest in the 11-20-year age category, higher in ages 21-30 years, and highest in ages 31-60 years (Fig. 4A). The change in cortisol over time varied within individual: two bulls (Male3 and Male4) exhibited an increase in cortisol over time, one (Male1) exhibited a decrease, and in one (Male2) there was no significant change with increasing age (Table 11).

The interaction of age and musth (Fig. 3B-F) shows how cortisol concentrations change with age during musth and inter-musth periods. For all individuals and all bulls combined, the interaction of age and musth state was significant (Table 11). The rate of change was greater during musth than in inter-musth in Male1 (Fig. 4B), Male2 (Fig. 4C), and Male3 (Fig. 4D), with relatively little change in inter-musth concentrations. By contrast, Male4 (Fig. 3E) showed a higher rate of increase with age in inter-musth periods, indicating that inter-musth concentrations increased more dramatically over time. 
Table 11: Age as a predictor of adrenal GC activity.

Individual, age range of analysis, age variable (age, age category, interaction of age and cycle), effect size with standard error (SE), Wald statistic, and p-value from GLMMs; and relative effect of age on mean cortisol concentrations. Degrees of freedom (df) is 1 in all pair-wise comparisons.

\begin{tabular}{|c|c|c|c|c|c|c|}
\hline Individual & $\begin{array}{c}\text { Age } \\
\text { Range of } \\
\text { Analysis }\end{array}$ & Age Variable & Effect Size (SE) & Wald & $\mathbf{P}$ & Age Effect \\
\hline \multirow[t]{4}{*}{ Male1 } & \multirow[t]{4}{*}{$\sim 34-43$} & Age (years) & $-0.026(0.005)$ & 32.714 & $<0.001$ & Decreases \\
\hline & & $31-40$ (reference) & - & - & - & \\
\hline & & $41-50$ & $-0.110(0.029)$ & 14.189 & $<0.001$ & Lower \\
\hline & & $\begin{array}{l}\text { Interaction of } \\
\text { Age and Musth }\end{array}$ & $-0.019(0.009)$ & 4.346 & $\mathbf{0 . 0 3 7}$ & $\begin{array}{c}\text { Interaction is } \\
\text { significant }\end{array}$ \\
\hline \multirow[t]{5}{*}{ Male2 } & \multirow[t]{5}{*}{$32-54$} & Age (years) & $-0.002(0.001)$ & 2.079 & 0.149 & -- \\
\hline & & $31-40$ (reference) & - & - & - & \\
\hline & & $41-50$ & $-0.028(0.017)$ & 2.714 & 0.099 & -- \\
\hline & & $51-60$ & $-0.022(0.021)$ & 1.092 & 0.296 & -- \\
\hline & & $\begin{array}{l}\text { Interaction of } \\
\text { Age and Musth }\end{array}$ & $-0.006(0.002)$ & 5.605 & 0.018 & $\begin{array}{c}\text { Interaction is } \\
\text { significant }\end{array}$ \\
\hline \multirow[t]{4}{*}{ Male3 } & \multirow[t]{4}{*}{$\sim 34-44$} & Age (years) & $0.011(0.004)$ & 8.054 & 0.021 & Increases \\
\hline & & $31-40$ (reference) & - & - & - & \\
\hline & & $41-50$ & $0.113(0.023)$ & 24.856 & $<0.001$ & Higher \\
\hline & & $\begin{array}{l}\text { Interaction of } \\
\text { Age and Musth }\end{array}$ & $0.010(0.008)$ & 1.451 & 0.228 & -- \\
\hline \multirow[t]{5}{*}{ Male $4^{\mathrm{a}}$} & \multirow[t]{5}{*}{$15-32$} & Age (years) & $0.025(0.002)$ & 118.624 & $<0.001$ & Increases \\
\hline & & $11-20$ (reference) & - & - & - & \\
\hline & & $21-30$ & $0.087(0.026)$ & 11.171 & 0.001 & Higher \\
\hline & & $31-40$ & $0.306(0.042)$ & 53.358 & $<0.001$ & Higher \\
\hline & & $\begin{array}{l}\text { Interaction of } \\
\text { Age and Musth }\end{array}$ & $-0.023(0.005)$ & 22.151 & $<0.001$ & $\begin{array}{c}\text { Interaction is } \\
\text { significant }\end{array}$ \\
\hline \multirow{7}{*}{$\begin{array}{l}\text { All } \\
\text { elephants }\end{array}$} & \multirow[t]{7}{*}{$15-54$} & Age (years) & $0.004(0.011)$ & 19.502 & $<0.001$ & Increases \\
\hline & & $11-20$ (reference) & - & - & - & \\
\hline & & $21-30$ & $0.090(0.023)$ & 15.450 & $<0.001$ & Higher \\
\hline & & $31-40$ & $0.283(0.035)$ & 65.123 & $<0.001$ & Higher \\
\hline & & $41-50$ & $0.284(0.037)$ & 58.378 & $<0.001$ & Higher \\
\hline & & $51-60$ & $0.276(0.041)$ & 44.934 & $<0.001$ & Higher \\
\hline & & $\begin{array}{l}\text { Interaction of } \\
\text { Age and Musth }\end{array}$ & $-0.007(0.001)$ & 42.213 & $<0.001$ & $\begin{array}{c}\text { Interaction is } \\
\text { significant }\end{array}$ \\
\hline
\end{tabular}

-- No significant difference or interaction is not significant

${ }^{a}$ Age analysis includes only mature bulls, which starts at age 15 for this individual. 

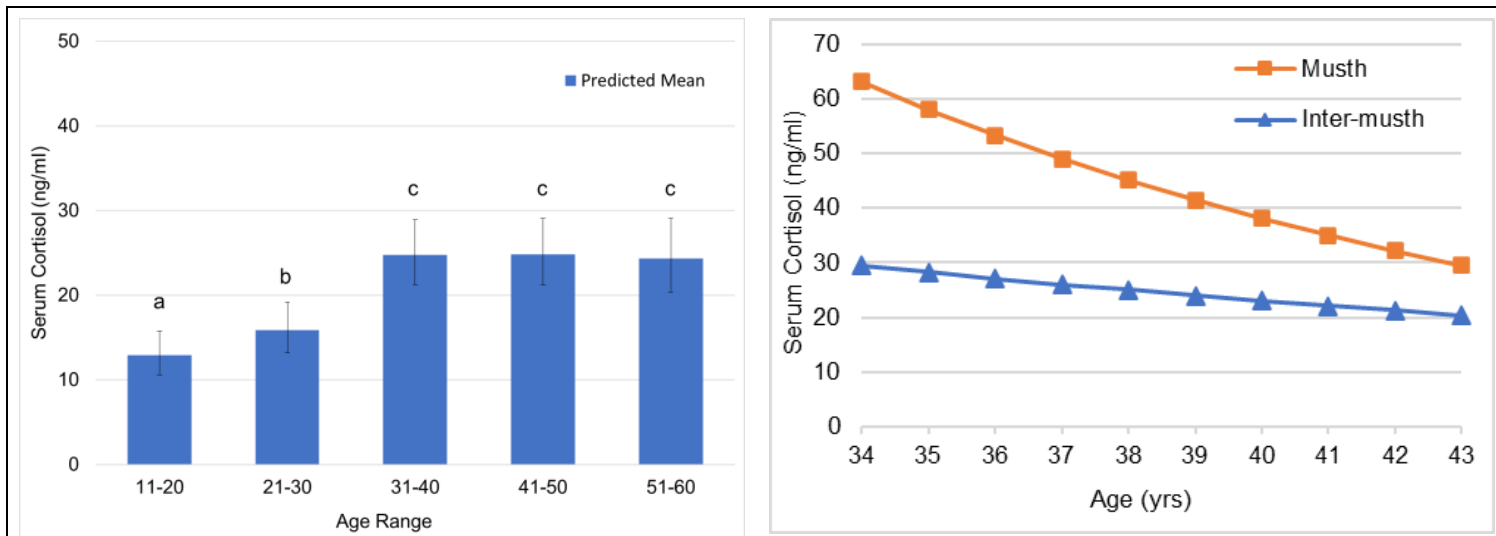

(A)

(B)

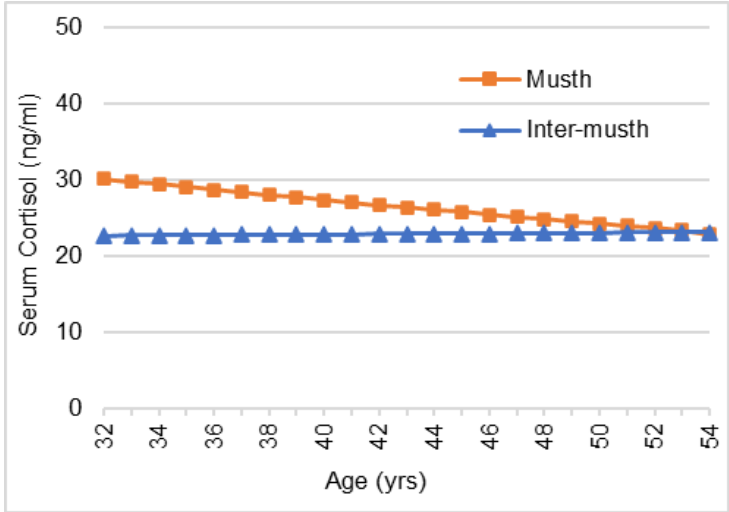

(C)

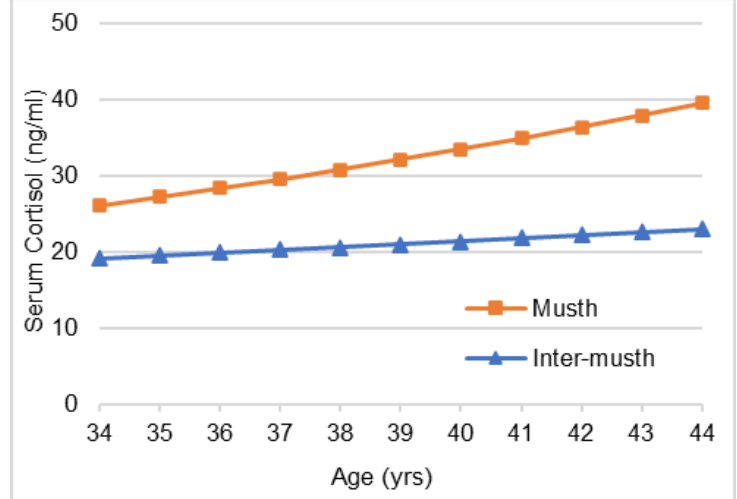

(D)
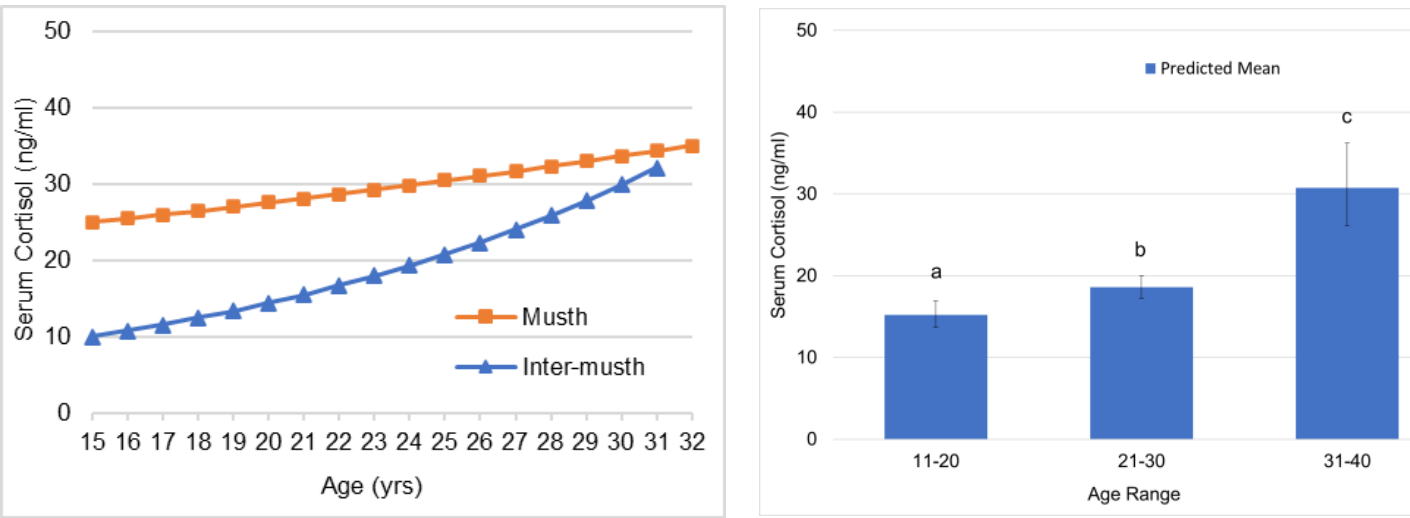

(E)

(F)

Fig 4: Age as a predictor of adrenal GC activity.

Predictions from GLMMs for serum cortisol concentration comparing mean values across age categories (error bars represent standard error of the prediction) (A and F), and interactions of age and musth state showing how cortisol during musth and inter-musth changes with age (B-E). Letters denote significant differences in mean values. (A) All elephants: Comparing mean values across age bins. (B) Male1: Interaction of age and musth state. (C) Male2: Interaction of age and musth state. (D) Male3: Interaction of age and musth state. (E) Male4: Interaction of age and musth state. (F) Male4: Comparing mean values across age bins. 


\section{Origin of birth}

When comparing cortisol concentrations between wild-born $(n=2)$ and zoo-born $(n=2)$

males, the youngest and oldest age categories were each comprised of only one individual and origin was confounded by age. This comparison was limited to the 31-50-year age groups ( $\mathrm{N}=1861$ samples), where there was no difference in mean serum cortisol concentrations between wild-born and zoo-born males $($ GLMM coefficient $=-0.031, \mathrm{SE}$ $\left.=0.068, \chi^{2}=0.211, \mathrm{df}=1, \mathrm{p}=0.646\right)$.

\section{Social life events}

During the study, one bull was transferred in from another facility, one bull died, and the other three were humanely euthanized. Table 12 shows the social life events experienced by each individual, comparing mean cortisol concentrations between post-event and preevent time periods, with covariates of musth included in the GLMM when there was a change in reproductive state within the timeframe of the event. The transfer of a bull into the facility had no significant effect on serum cortisol concentrations in any of the resident bulls. Death of a bull (Male1) was a significant predictor of mean cortisol concentration in only one individual (Male4). No other bulls responded to the death of another bull with a change in cortisol secretion. 
Table 12: Social life events as predictors of adrenal GC activity.

Fixed effects, effect size with standard error (SE), Wald statistic, and p-value from GLMMs, and whether the mean cortisol concentration post-event was higher or lower than pre-event. Reference category is preevent, and comparison category is post-event. Degrees of freedom (df) was 1 in all pair-wise comparisons.

\begin{tabular}{|c|c|c|c|c|c|}
\hline Fixed Effect & $\begin{array}{c}\text { Individual } \\
\text { Exposed to } \\
\text { Event }\end{array}$ & Effect Size (SE) & Wald & $\mathbf{P}$ & $\begin{array}{l}\text { Mean Post-event } \\
\text { Relative to } \\
\text { Pre-event }\end{array}$ \\
\hline \multirow{2}{*}{$\begin{array}{l}\text { Transfer in of Male } 3 \\
\text { (30 days pre/post) }\end{array}$} & Male2 & $0.20090 .240)$ & 0.697 & 0.404 & -- \\
\hline & Male4 & $0.038(0.052)$ & 0.528 & 0.467 & -- \\
\hline \multirow{2}{*}{$\begin{array}{l}\text { Transfer in of Male } 3 \\
\text { (60 days pre/post) }\end{array}$} & Male2 & $0.146(0.201)$ & 0.527 & 0.468 & -- \\
\hline & Male4 & $0.014(0.182)$ & 2.006 & 0.157 & -- \\
\hline \multirow{2}{*}{$\begin{array}{l}\text { Death of Male1 } \\
\text { (30 days pre/post) }\end{array}$} & Male2 & $-0.240(0.205)$ & 1.375 & 0.241 & -- \\
\hline & Male4 & $-0.396(0.183)$ & 4.678 & 0.031 & -- \\
\hline \multirow{2}{*}{$\begin{array}{l}\text { Death of Male1 } \\
\text { (60 days pre/post) }\end{array}$} & Male2 & $-0.168(0.193)$ & 0.757 & 0.384 & -- \\
\hline & Male4 & $-0.427(0.129)$ & 11.017 & 0.001 & Lower \\
\hline \multirow{2}{*}{$\begin{array}{l}\text { Euthanasia of Male4 } \\
\text { (30 days pre/post) }\end{array}$} & Male2 & $-0.183(0.129)$ & 2.002 & 0.157 & -- \\
\hline & Male3 & $-0.153(0.221)$ & 0.480 & 0.489 & -- \\
\hline \multirow{2}{*}{$\begin{array}{l}\text { Euthanasia of Male4 } \\
\text { (60 days pre/post) }\end{array}$} & Male2 & $0.060(0.090)$ & 0.454 & 0.500 & -- \\
\hline & Male3 & $-0.160(0.126)$ & 0.613 & 0.204 & -- \\
\hline $\begin{array}{l}\text { Euthanasia of Male3 } \\
\text { (30 days pre/post) }\end{array}$ & Male2 & $-0.050(0.137)$ & 0.135 & 0.713 & -- \\
\hline $\begin{array}{l}\text { Euthanasia of Male } 3 \\
\text { ( } 60 \text { days pre/post) }\end{array}$ & Male2 & $-0.016(0.111)$ & 0.022 & 0.882 & -- \\
\hline
\end{tabular}

-- No significant difference

\section{Mycobacterium tuberculosis detection and treatment}

Table 13 shows comparisons of mean cortisol concentrations between time periods

preceding detection of Mycobacterium tuberculosis $(M . t b)$ by positive TW culture, and time periods before and after anti-TB therapy start and end, with covariates of musth included in the GLMM when there was a change in musth state within the timeframe of the period tested. 
In Male3 and Male2, the period of transition from the last culture-negative TW to detection of active $M$. tb. was 9 weeks and 10 weeks, respectively. Therefore, comparisons in cortisol concentrations were made between 30-day time blocks in the 120 days preceding $M . t b$. detection. In the first bull diagnosed, Male4, the period of transition from the last culture-negative TW to detection of active $M$. $t b$. was 14 months. Seroconversion also occurred within that time period, so comparisons in cortisol concentrations were made between this year-long transition period to the year prior, and also in the 120 days preceding detection.

Detection of active TB was a significant predictor of mean cortisol concentrations only in Male3, with higher cortisol 31-60 days prior to detection. Neither Male4 nor Male2 showed significant changes in mean cortisol concentrations with the transition to active TB. The start of anti-TB therapy was a significant predictor of mean cortisol concentrations only in Male3, with higher cortisol in the 30 days after starting therapy. Male2 was the only bull not undergoing anti-TB therapy at the time of euthanasia, and mean cortisol concentrations decreased in the 30 days after therapy ended. 
Table 13: TB detection and anti-TB therapy as predictors of adrenal GC activity.

Fixed effects, effect categories, effect size with standard error (SE), Wald statistic, and p-value from GLMMs, and whether the mean cortisol concentration of a time period was higher or lower relative to the reference time period. Degrees of freedom (df) was 1 in all pair-wise comparisons.

\begin{tabular}{|c|c|c|c|c|c|}
\hline Fixed Effect & Category & Effect Size (SE) & Wald & $\mathbf{P}$ & $\begin{array}{c}\text { Mean Relative } \\
\text { to Reference } \\
\text { Category }\end{array}$ \\
\hline \multirow[t]{4}{*}{$\begin{array}{l}\text { Male4: Detection of } \\
\text { active TB }\end{array}$} & $\begin{array}{l}\text { Prior to detection: } \\
\text { days } 120-91^{\text {a }}\end{array}$ & - & - & - & \\
\hline & $\begin{array}{l}\text { Prior to detection: } \\
\text { days } 90-61\end{array}$ & $-0.039(0.130)$ & 0.092 & 0.762 & -- \\
\hline & $\begin{array}{l}\text { Prior to detection: } \\
\text { days 31-60 }\end{array}$ & $-0.144(0.130)$ & 1.236 & 0.266 & -- \\
\hline & $\begin{array}{l}\text { Prior to detection: } \\
\text { days 0-30 }\end{array}$ & $-0.014(0.130)$ & 0.011 & 0.916 & -- \\
\hline \multirow[t]{2}{*}{$\begin{array}{l}\text { Male4: Detection of } \\
\text { active TB }\end{array}$} & $\begin{array}{l}\text { Prior to detection: } \\
\text { year } 1-2^{\text {a }}\end{array}$ & - & - & - & \\
\hline & $\begin{array}{l}\text { Prior to detection: } \\
\text { year } 0-1\end{array}$ & $0.082(0.049)$ & 2.816 & 0.093 & -- \\
\hline \multirow[t]{2}{*}{$\begin{array}{l}\text { Male4: Start of } \\
\text { anti-TB therapy }\end{array}$} & $\begin{array}{l}\text { Prior to therapy start: } \\
30 \text { days }^{\mathrm{a}}\end{array}$ & - & - & - & \\
\hline & $\begin{array}{l}\text { Post therapy start: } \\
30 \text { days }\end{array}$ & $-0.127(0.148)$ & 0.743 & 0.389 & -- \\
\hline \multirow[t]{4}{*}{$\begin{array}{l}\text { Male3: Detection of } \\
\text { active TB }\end{array}$} & $\begin{array}{l}\text { Prior to detection: } \\
\text { days } 120-91^{\text {a }}\end{array}$ & - & - & - & \\
\hline & $\begin{array}{l}\text { Prior to detection: } \\
\text { days } 90-61^{\text {b }}\end{array}$ & $-0.122(0.119)$ & 1.041 & 0.308 & -- \\
\hline & $\begin{array}{l}\text { Prior to detection: } \\
\text { days } 31-60^{\mathrm{b}}\end{array}$ & $0.469(0.146)$ & 5.607 & 0.019 & Higher \\
\hline & $\begin{array}{l}\text { Prior to detection: } \\
\text { days 0-30 }\end{array}$ & $0.157(0.146)$ & 1.160 & 0.281 & -- \\
\hline \multirow[t]{2}{*}{$\begin{array}{l}\text { Male3: Start of } \\
\text { anti-TB therapy }\end{array}$} & $\begin{array}{l}\text { Prior to therapy start: } \\
30 \text { days }{ }^{\text {a }}\end{array}$ & - & - & - & \\
\hline & $\begin{array}{l}\text { Post therapy start: } \\
30 \text { days }\end{array}$ & $0.222(0.122)$ & 3.331 & 0.068 & Higher \\
\hline \multirow[t]{3}{*}{$\begin{array}{l}\text { Male2: Detection of } \\
\text { active TB }\end{array}$} & $\begin{array}{l}\text { Prior to detection: } \\
\text { days } 120-91^{\text {a }}\end{array}$ & - & - & - & \\
\hline & $\begin{array}{l}\text { Prior to detection: } \\
\text { days } 90-61\end{array}$ & $0.205(0.168)$ & 1.486 & 0.223 & -- \\
\hline & $\begin{array}{l}\text { Prior to detection: } \\
\text { days } 31-60\end{array}$ & $0.169(0.168)$ & 1.013 & 0.314 & -- \\
\hline
\end{tabular}




\begin{tabular}{|l|l|c|c|c|c|}
\hline \multicolumn{1}{|c|}{ Fixed Effect } & \multicolumn{1}{|c|}{ Category } & Effect Size (SE) & Wald & P & $\begin{array}{c}\text { Mean Relative } \\
\text { to Reference } \\
\text { Category }\end{array}$ \\
\hline & $\begin{array}{l}\text { Prior to detection: } \\
\text { days 0-30 }\end{array}$ & $0.201(0.168)$ & 1.421 & 0.233 & -- \\
\hline $\begin{array}{l}\text { Male2: Start of } \\
\text { anti-TB therapy }\end{array}$ & $\begin{array}{l}\text { Prior to therapy start: } \\
\text { 30 days a }\end{array}$ & - & - & - & \\
\cline { 2 - 7 } & $\begin{array}{l}\text { Post therapy start: } \\
30 \text { days }\end{array}$ & $-0.093(0.137)$ & 0.465 & 0.495 & - \\
\hline $\begin{array}{l}\text { Male2: End of } \\
\text { anti-TB therapy }\end{array}$ & $\begin{array}{l}\text { Prior to therapy end: } \\
30 \text { days }{ }^{\text {a }}\end{array}$ & - & - & - & \\
\cline { 2 - 7 } & $\begin{array}{l}\text { Post therapy end: } \\
30 \text { days }\end{array}$ & $-0.354(0.133)$ & 7.046 & $\mathbf{0 . 0 0 8}$ & Lower \\
\hline
\end{tabular}

-- No significant difference

${ }^{a}$ denotes the reference category for GLMMs

${ }^{\mathrm{b}}$ denotes significant difference between time periods in post-hoc comparisons

\section{Health decline}

The bull that died of sepsis (Male1) showed higher mean cortisol concentration in the final 30 days of life (Table 14), with a sharp increase in the 4 days prior to death. In the three males that were euthanized, there were no significant differences in mean cortisol between the 30-day time period of health decline preceding euthanasia and the previous 30 days.

Table 14: Health decline leading to natural death or euthanasia as a predictor of adrenal GC activity. Fixed effects, effect size with standard error (SE), Wald statistic, and p-value from GLMMs, and whether the mean cortisol concentration in the final 30 days prior to death/euthanasia was higher or lower than the previous 30 days. Comparison category is the final 30 days, and reference category is the previous 30 days. Degrees of freedom (df) was 1 in all pair-wise comparisons.

\begin{tabular}{|l|c|c|c|c|}
\hline \multicolumn{1}{|c|}{ Fixed Effect } & Effect Size (SE) & Wald & P & $\begin{array}{c}\text { Mean } \\
\text { Final 30 Days Relative to } \\
\text { Previous 30 Days }\end{array}$ \\
\hline Death of self: Male1 & $0.548(0.211)$ & 6.753 & $\mathbf{0 . 0 0 9}$ & Higher \\
\hline Euthanasia: Male4 & $-0.075(0.155)$ & 0.236 & 0.627 & -- \\
\hline Euthanasia: Male3 & $0.015(0.199)$ & 0.005 & 0.994 & -- \\
\hline Euthanasia: Male2 & $0.019(0.081)$ & 0.056 & 0.813 & -- \\
\hline
\end{tabular}

-- No significant difference 


\section{Discussion}

This study confirmed co-secretory patterns of cortisol and testosterone during musth in Asian elephant bulls, and highlights the importance of accounting for intrinsic glucocorticoid (GC) secretion patterns when evaluating the impact of social or environmental changes on adrenal GC activity. Testosterone decreased with age in all adult bulls, whereas age-related patterns in cortisol varied across individuals. Acute adrenal responses in association with anti-TB therapy were exhibited in two bulls. Except for a significant end of life cortisol increase in one bull, there was no clear evidence of prolonged elevated cortisol secretion outside of musth in any individual. In contrast to influences of physiological changes such as underlying changes involved in musth cycles, there was no evidence that social changes with the addition or removal of bulls impacted adrenal GC activity in the short-term.

\section{$\underline{\text { Longitudinal serum testosterone patterns }}$}

\section{Musth}

The bulls in this study exhibited regular annual musth cycles, each occurring at different times of year with no evidence of synchronization, but with varying musth durations and more overlap in some years such that multiple bulls were in musth at the same time. There were no extended periods of elevated testosterone outside of musth, however all bulls experienced testosterone elevations of short duration that in some cases also coincided with behavioral and physical correlates (i.e., aggression, TGS/UD) of musth. These episodic elevations were associated with a recurrence of musth, with musth cycles 
of other bulls, and presence of estrous females and/or breeding activity. Brief elevations in testosterone associated with presence of estrous females and mating were also found in previous studies (Niemuller and Liptrap, 1991; Rasmussen et al., 2008; Scallan et al., 2011). Although the elephants in this study were fairly consistent in their annual musth cycles for much of their lives, variability both within and across individuals highlights the need to closely and consistently monitor each individual from an early age and throughout musth and inter-musth periods to determine the pattern for each bull for proper and safe management.

Contrary to the expression of annual musth in this study, Scott and Riddle (2003) and LaDue et al. (2014) reported that musth in captivity varied from musth exhibited in freeranging elephants in that a large proportion of adult males in captivity experienced musth more than once a year, and age of the individual or of any other males was not a reliable indicator of musth duration or frequency. Furthermore, not all bulls of adequate age exhibit musth in North American zoos (Brown et al., 2007; Chave et al., 2019), and in addition to the housing of multiple adult bulls rather than single bulls, perhaps the age structure of the bulls is a factor influencing musth expression. Keerthipriya and Vidya (2019) found in wild Asian elephants that the presence of males in the oldest age class of (45+ in that study) seemed to positively affect the overall occurrence of musth. Taking this a step further, Hartley et al. (2019) suggests even broader considerations for social management of male elephants to better provide for their social needs across their life span. 


\section{Musth threshold concentrations}

Testosterone concentrations corresponding to the beginning and end of musth suggest that threshold concentrations are individualistic. Although the minimum concentration defines the absolute threshold, the median and mean represented more typical concentrations defining full musth, and the median and mean were closer to the $50 \mathrm{ng} / \mathrm{ml}$ threshold found by Brown et al. (2007) in captive Asian and African bulls. Furthermore, the minimum value for the first musth point defined by Chave et al. (2019) corresponds more closely to the mean than the minimum, suggesting the mean is a more appropriate measure of musth threshold concentrations.

\section{Effects of age and dominance status on testosterone across bulls}

Higher overall testosterone concentrations with social rank have been reported in captive (Jainudeen et al., 1972b; Lincoln and Ratnasooriya, 1996) and wild elephants (HallMartin and Van der Walt, 1984); however, there were no definitive relationships between higher mean or musth threshold testosterone concentrations and age or dominance rank among these bulls. This is in contrast to age-related increases in mean testosterone found in epidemiological studies (Brown et al., 2007; Chave et al., 2019). However, these studies included males that had not exhibited musth, and a larger number of older males exhibited musth compared to younger males, so the age-related increase is in part due to the differential expression of musth. Thongtip et al. (2008) found age-related decreases in circulating and seminal testosterone across Asian elephant bulls during inter-musth periods, with testosterone being highest in the 10-19-year age group, followed by the 2343-year age group, then lowest in the 51-70-year age group. 


\section{Effects of age on testosterone within individuals}

Testosterone decreased with age in all adult bulls, as measured by comparing mean concentrations with age and across age categories, and by visual inspection of hormone profiles. The youngest male showed increased testosterone with age in the 5 years after his first musth cycle, and then a gradual decrease. In younger males, expression of musth is shorter and more sporadic, becoming more robust with increasing age (Poole, 1987, 1989b; Lincoln and Ratnasooriya, 1996; Rasmussen et al., 2002). All older bulls showed decreased mean testosterone past 31-40 years of age, providing evidence of age-related decreases in testosterone similar to that found in male model rats (Wang et al., 1993) and humans (Bremner et al., 1983; Morley et al., 1997).

\section{Effects of puberty, musth, and testosterone on cortisol}

Serum cortisol covaried positively with testosterone and musth as observed in previous studies (Brown et al., 2007; Yon et al., 2007; Chave et al., 2019). While the significance of increased adrenal GC activity during musth has not been determined, Yon et al. (2007) provide evidence that the adrenal glands increase steroid production in general during musth, as measured by increases in cortisol, dehydroepiandrosterone (DHEA), and androstenediol. Explanations for the increase in cortisol during musth include the possibility that the state of musth is energetically costly and presents a physiological challenge (Rasmussen and Perrin, 1999; Yon et al., 2007). It also may be associated with ecological stress, and increased secretory activity may help animals cope with these challenges. Cortisol may also increase in response due to high metabolic demand and reduced food intake as found in other mammalian species (Muller and Wrangham, 2004). 
It should be noted that findings of increased circulating cortisol during musth in our study and others (Brown et al., 2007; Yon et al., 2007; Chave et al., 2019) are in sharp contrast to studies showing excreted fecal GC metabolites (FGMs) are not elevated during periods of musth in captive and free-ranging African (Ganswindt et al., 2003; Ganswindt et al., 2005a; Ganswindt et al., 2005b; Ganswindt et al., 2010) and free-ranging Asian (Ghosal et al., 2013) elephant bulls. Kumar et al. (2014) showed increased FGMs during musth in captive Asian bulls, but suggested the increase might be due to musth management practices rather than the physiological state of musth. One obvious difference between these studies is the measurement of circulating versus excreted GCs. From a metabolic perspective, it seems plausible that metabolic changes that occur during musth (Rasmussen and Perrin, 1999) could influence concentrations in excreted steroids. Bulls in musth may spend less time feeding, and zoos report voluntary food reduction, inappetence and weight loss for bulls in musth (Rasmussen and Perrin, 1999). Changes in food availability have been shown to trigger large fluctuations in metabolic rate of freeliving animals (Goymann, 2012). It is also possible this could affect fecal GC metabolite concentrations as there is no reliable method for indexing steroid concentrations to account for fluctuations in food intake. In other ungulates, the analogous condition of rut has been shown to be a metabolically challenging period for males with active selection for those in good condition (Barboza et al., 2004), and has been associated with increases in fecal GC metabolites (Khonmee et al., 2014). Metrione et al. (2020) observed lower FGMs in bison during winter months when their metabolic rate is also low, suggesting a metabolic strategy in which glucocorticoids are minimally secreted in order to conserve 
energy when forage is scarce. Clearly, further studies are needed to elucidate how adrenal GC activity is altered in relation to metabolic changes and the physiological changes associated with musth.

\section{Effects of age on cortisol across bulls}

Overall, mean cortisol concentrations increased with age with all adult bulls combined, being lowest in the 11-20-year age group which included early musth cycles in the youngest bull, higher in 21-30-year age group, and highest in ages 31-60 years. This overall increase with respect to age across bulls was in agreement with previous studies (Brown et al., 2007; Chave et al., 2019).

\section{Effects of age on cortisol within individuals}

Although cortisol covaried with testosterone and increased during musth in all bulls, agerelated changes in cortisol concentrations indicated that the amount of cortisol increase during musth was changing over time. Age-related changes in cortisol concentrations varied across individuals, with an increase in cortisol over time in two bulls, a decrease in one bull, and no significant change in one bull.

The interaction between age and musth state was significant in all bulls, indicating concentrations changed differentially during musth versus inter-musth periods with age. The youngest male exhibited a higher rate of change in inter-musth concentrations, with an increase of approximately $20 \mathrm{ng} / \mathrm{ml}$, more than double the change in inter-musth concentrations in the older males, which could have been related in part to the 
exacerbation of his joint issues (Brown et al., 2019; Edwards et al., 2019b). The three older bulls exhibited the greatest magnitude of change during musth, with much smaller changes in inter-musth concentrations. However, the pattern of change differed across bulls, which could be related to social factors, musth, or simply individual differences. Male1 and Male3 were the same age during the study; Male1 showed cortisol concentrations during musth decreased over time, and he was with females only infrequently for breeding and only in the first half of the study; whereas Male3 showed cortisol concentrations that increased during musth over time, and he was breeding and integrated with the female herd with frequent social opportunities. Interestingly, Male2 showed a narrower range in cortisol concentrations between musth and inter-musth periods than other bulls, which narrowed further over time such that mean concentrations did not differ between musth and inter-musth periods. It is plausible that this merging of musth and inter-musth cortisol values could be related to Male2's erratic musth cycles in the last few years of his life. The role of cortisol during musth requires further investigation to explore age-related differences, and whether any changes could be related to social dynamics.

\section{Effects of origin of birth on cortisol}

Mean cortisol concentrations did not differ significantly between wild-born and zoo-born males with the comparison limited to the 31-50 age group. However, another important factor is life history, and the wild-born males in this study experienced multiple inter-zoo transfers, whereas one zoo-born male experienced only two transfers and the other spent his entire life at the Oregon Zoo. In other species, cortisol secretion later in life can be 
influenced by early life trauma (McEwen and Wingfield, 2003) or early social experience influencing development of the stress response (Hawkley et al., 2012; Creel et al., 2013). Prado-Oviedo et al. (2016) reported differences in the early lives of wild-born versus captive-born elephants that could have long-term welfare implications; and Greco et al. (2016) suggests that factors such as travel, unfamiliar surroundings, and social separation may explain higher rates of stereotypic behavior associated with number of transfers.

\section{Effects of social life events on cortisol}

Social changes with the addition of a new adult bull and deaths of other bulls had no apparent impact on mean cortisol concentrations in resident males. Although housed separately from each other, bulls did have olfactory and acoustic contact, and it was presumed they had hormonal influences on each other, which was supported by testosterone increases of short duration that coincided with other bulls in musth. Social factors can influence musth expression in bulls (Brown et al., 2007). Perhaps if social factors had presented significant psychological stressors leading to chronic GC elevation for any individual, then we might expect that individual to show a short-term decrease in cortisol with the death of another bull depending on dominance rank. However, there was no evidence of chronically elevated or depressed GC levels in these bulls, and no significant adrenal response to major social changes.

\section{Mycobacterium tuberculosis detection and treatment}

Although cortisol has been shown to increase in association with pathologies such as gastrointestinal issues, skin lesions, lameness, foot lesions (Edwards et al., 2019b), an increase associated with activation of Mycobacterium tuberculosis disease was observed 
in only one individual, suggesting cortisol is not a good biomarker for identifying an immune response triggered by cytokines (Bottasso et al., 2007) in the transition from latent to active TB.

The increase in cortisol after starting anti-TB therapy in at least one individual, and the decrease after ending therapy supports caretaker accounts that the onset of and recovery from adverse treatments side effects (Rosen et al., 2019) was quick. These adrenal responses suggest that GC monitoring may provide an additional tool for assessing wellbeing during certain medical treatments. In Male2, age-related changes in musth characteristics made treatment with oral medications even more challenging. One of his typical musth symptoms was inappetence. Therefore, treatment became more difficult when his musth episodes became more erratic and he was showing musth signs more frequently. Elephant care staff developed a feeding regimen to manage the intensity of his musth, allowing him to exhibit only a "mild" musth, which helped ensure his cooperation with keeper staff for administration of oral medication and continued therapy.

\section{$\underline{\text { Conclusions }}$}

This longitudinal study contributes to the sustainability of the managed Asian elephant populations by advancing our knowledge of individual variabilty in bull reproductive physiology. Musth is a condition that can create both human safety and animal welfare concerns. It is therefore paramount, for improved bull management, to understand the physiological changes associated with musth and to have tools available for assessing the well-being of male elephants of all ages. Findings from this study have implications for 
management of male elephants. This longitudinal analysis further elucidated age-related changes in testosterone, but the study results also emphasize the need for further investigations into the role of GCs in the physiology of musth. Hormone secretion patterns measured in this study highlight the importance of taking intrinsic patterns of reproductive hormones into account when assessing adrenal response to external stimuli (see also Chapter 2). Cortisol has been shown to increase with end of life health declines in some individuals (e.g., Chapter 2), and this was the case in the one bull that died of sepsis, but not in the two bulls that were euthanized due to joint pathologies. The fourth bull that was euthanized due to development of a drug-resistant strain of M.tb., but was not exhibiting signs of health decline (Miller et al., 2018). The differential response may in part be due to the point in decline at which the euthanasia decision was made, or to the nature of the health decline itself. Although the cortisol data provided no evidence of chronic stress, given medical treatment perceived by caretakers to impact general wellbeing, and health decline with physical discomfort, it might be beneficial to include measures of immunoglobulin-A (IgA) as a potential biomarker of stress and positive or negative affective state to help inform health and 'end of life' decision making. IgA is an immune protein associated with pathogen defence, and has been demonstrated to decrease during times of stress and increase in response to positive stimuli [reviewed in Edwards et al. (2019a)]. Including IgA as a biomarker would contribute to a more comprehensive assessment of welfare with consideration of both positive and negative aspects. 
Age-related changes in dehydroepiandrosterone (DHEA), an adrenal hormone shown to increase in musth (Yon et al., 2007) and decrease with advanced age in humans (Samaras et al., 2013), could provide insight into the relationship between adrenal GC activity and metabolic changes, and aid in the care of geriatric elephants.

Results from this study will also be useful in ongoing research to investigate immune function during musth. Testosterone has been shown in other species to have immunosuppressive effects (Pérez-Rodríguez et al., 2006). It is therefore possible that the occurrence of musth could be associated with immunosuppression in bull elephants, which combined with a decline in food intake and behavior changes, could have important implications for TB management in bull elephants. Lastly, we continue to learn from these bulls, as this dataset and banked serum are still part of ongoing studies to identify additional biomarkers, such as those associated with inflammation and immune function, which can be used for assessing how environmental and physiological factors affect an animal's health over a period of time.

\section{Acknowledgements}

We thank the dedicated Oregon Zoo elephant care staff who collected blood samples and tracked musth for so many years to aid in management and scientific advancement: Jeb Barsh, Tarah Bedrossian, Dimas Dominguez, Shawn Finnell, Pat Flora, Gilbert Gomez, Doug Groves, Jay Haight, Roger Henneous, Ray Hopper, David Illig, Mike Keele, Jeremy Kirby, Bob Lee, Fred Marion, Sioux Marion, Jason Miles, Phil Prewett, Charlie Rutkowski, Joseph Sabastiani, Jim Sanford, Pam Starkey, and April Yoder. We owe the 
integrity of the hormone dataset to the Oregon Zoo endocrinology lab managers and technicians: Carrie Hays, David Hess, Karen Lewis, Meghan Martin, Camie Meller, Anne Schmidt, Michael Schmidt, Nancy Scott, Barbara Slade. Funding for all hormone analyses was generously provided by the Oregon Zoo, Smithsonian Conservation Biological Institute Endocrinology Lab, and additional grant support from the Portland State University Forbes-Lea Endowment Fund. Finally, many thanks to the Oregon Zoo registrar Peter Grimm for retrieval of musth records, saving many hours of manual review.

\section{Literature Cited}

Barboza, P. S., D. W. Hartbauer, W. E. Hauer, and J. E. Blake. 2004. Polygynous mating impairs body condition and homeostasis in male reindeer (Rangifer tarandus tarandus). J Comp Physiol B 174(4):309-317. doi: 10.1007/s00360-004-0416-6

Bolker, B. M., M. E. Brooks, C. J. Clark, S. W. Geange, J. R. Poulsen, M. H. H. Stevens, and J.-S. S. White. 2009. Generalized linear mixed models: a practical guide for ecology and evolution. Trends Ecol Evol 24(3):127-135. doi: https://doi.org/10.1016/j.tree.2008.10.008

Borg, K. E., K. L. Esbenshade, and B. H. Johnson. 1991. Cortisol, growth hormone, and testosterone concentrations during mating behavior in the bull and boar. J Anim Sci 69(8):3230-3240. doi: 10.2527/1991.6983230x \%J Journal of Animal Science

Bottasso, O., M. L. Bay, H. Besedovsky, and A. Del Rey. 2007. The Immuno-endocrine Component in the Pathogenesis of Tuberculosis. 66(2-3):166-175. doi: 10.1111/j.1365-3083.2007.01962.x

Boyle, S. A., B. Roberts, B. M. Pope, M. R. Blake, S. E. Leavelle, J. J. Marshall, A. Smith, A. Hadicke, J. F. Falcone, K. Knott, and A. J. Kouba. 2015. Assessment of Flooring Renovations on African Elephant (Loxodonta africana) Behavior and Glucocorticoid Response. PLOS ONE 10(11):e0141009. doi: 10.1371/journal.pone.0141009 
Bremner, W. J., M. V. Vitiello, and P. N. Prinz. 1983. Loss of Circadian Rhythmicity in Blood Testosterone Levels with Aging in Normal Men. The Journal of Clinical Endocrinology \& Metabolism 56(6):1278-1281. doi: 10.1210/jcem-56-6-1278 \%J The Journal of Clinical Endocrinology \& Metabolism

Brown, J. L., M. Bush, D. E. Wildt, J. R. Raath, V. d. Vos, and J. G. Howard. 1993. Effects of $\mathrm{GnRH}$ analogues on pituitary-testicular function in free-ranging African elephants (Loxodonta africana). Journal of Reproduction and Fertility 99(2):627. doi: 10.1530/jrf.0.0990627

Brown, J. L., K. Carlstead, J. D. Bray, D. Dickey, C. Farin, and K. Ange-van Heugten. 2019. Individual and environmental risk factors associated with fecal glucocorticoid metabolite concentrations in zoo-housed Asian and African elephants. PLoS One 14(9):e0217326. doi: https://doi.org/10.1371/journal.pone.0217326

Brown, J. L., D. C. Kersey, E. W. Freeman, and T. Wagener. 2010. Assessment of diurnal urinary cortisol excretion in Asian and African elephants using different endocrine methods. Zoo Biol 29(2):274-283. doi: https://doi.org/10.1002/zoo.20268

Brown, J. L., and J. Lehnhardt. 1995. Serum and urinary hormones during pregnancy and the peri- and postpartum period in an Asian elephant (Elephas, maximus). Zoo Biol 14(6):555-564. doi: https://doi.org/10.1002/zoo.1430140608

Brown, J. L., M. Somerville, H. S. Riddle, M. Keele, C. K. Duer, and E. W. Freeman. 2007. Comparative endocrinology of testicular, adrenal and thyroid function in captive Asian and African elephant bulls. Gen Comp Endocrinol 151(2):153-162. doi: http://dx.doi.org/10.1016/j.ygcen.2007.01.006

Brown, M. B., and A. B. Forsythe. 1974. Robust Tests for the Equality of Variances. J Am Stat Assoc 69(346):364-367. doi: https://doi.org/10.1080/01621459.1974.10482955

Burks, K. D., J. D. Mellen, G. W. Miller, J. Lehnhardt, A. Weiss, A. J. Figueredo, and T. L. Maple. 2004. Comparison of two introduction methods for African elephants (Loxodonta africana). Zoo Biol 23(2):109-126. doi: https://doi.org/10.1002/zoo.10132

Busch, D. S., and L. S. Hayward. 2009. Stress in a conservation context: A discussion of glucocorticoid actions and how levels change with conservation-relevant variables. Biol Conserv 142(12):2844-2853. doi: https://doi.org/10.1016/j.biocon.2009.08.013 
Buwalda, B., J. Scholte, S. F. de Boer, C. M. Coppens, and J. M. Koolhaas. 2012. The acute glucocorticoid stress response does not differentiate between rewarding and aversive social stimuli in rats. Horm Behav 61(2):218-226. doi: https://doi.org/10.1016/j.yhbeh.2011.12.012

Chave, E., K. L. Edwards, S. Paris, N. Prado, K. A. Morfeld, and J. L. Brown. 2019. Variation in metabolic factors and gonadal, pituitary, thyroid, and adrenal hormones in association with musth in African and Asian elephant bulls. Gen Comp Endocrinol 276:1-13. doi: https://doi.org/10.1016/j.ygcen.2019.02.005

Cooper, K. A., J. D. Harder, D. H. Clawson, D. L. Fredrick, G. A. Lodge, H. C. Peachey, T. J. Spellmire, and D. P. Winstel. 1990. Serum testosterone and musth in captive male African and Asian elephants. Zoo Biology 9(4):297-306. doi: 10.1002/zoo.1430090405

Creel, S., B. Dantzer, W. Goymann, and D. R. Rubenstein. 2013. The ecology of stress: effects of the social environment. Funct Ecol 27(1):66-80. doi: https://doi.org/10.1111/j.1365-2435.2012.02029.x

Dag, O., A. Dolgun, and N. M. Konar. 2018. onewaytests: An R Package for One-Way Tests in Independent Groups Designs. The R Journal 10:175-199. doi: Available from: https://journal.r-project.org/archive/2018/RJ-2018-022/index.html

Dathe, H. H., B. Kuckelkorn, and D. Minnemann. 1992. Salivary cortisol assessment for stress detection in the Asian elephant (Elephas maximus): A pilot study. Zoo Biology 11(4):285-289. doi: 10.1002/zoo.1430110408

Dayton, A., E. C. Exner, J. D. Bukowy, T. J. Stodola, T. Kurth, M. Skelton, A. S. Greene, and A. W. Cowley, Jr. 2016. Breaking the Cycle: Estrous Variation Does Not Require Increased Sample Size in the Study of Female Rats. Hypertension 68(5):1139-1144. doi: https://doi.org/10.1161/HYPERTENSIONAHA.116.08207

Edwards, K. L., P. Bansiddhi, S. Paris, M. Galloway, and J. L. Brown. 2019a. The development of an immunoassay to measure immunoglobulin A in Asian elephant feces, saliva, urine and serum as a potential biomarker of well-being. Conserv Physiol 7(1):coy077. doi: https://doi.org/10.1093/conphys/coy077

Edwards, K. L., M. A. Miller, K. Carlstead, and J. L. Brown. 2019b. Relationships between housing and management factors and clinical health events in elephants in North American zoos. PLoS One 14(6):e0217774. doi: 10.1371/journal.pone.0217774 
Edwards, K. L., S. Shultz, M. Pilgrim, and S. L. Walker. 2015. Irregular ovarian activity, body condition and behavioural differences are associated with reproductive success in female eastern black rhinoceros (Diceros bicornis michaeli). Gen Comp Endocrinol 214:186-194. doi: https://doi.org/10.1016/j.ygcen.2014.07.026

Edwards, K. L., J. Trotter, M. Jones, J. L. Brown, H. W. Steinmetz, and S. L. Walker. 2016. Investigating temporary acyclicity in a captive group of Asian elephants (Elephas maximus): Relationship between management, adrenal activity and social factors. Gen Comp Endocrinol 225:104-116. doi: http://dx.doi.org/10.1016/j.ygcen.2015.09.011

Eisenberg, J. F., G. M. McKay, and M. R. Jainudeen. 1971. Reproductive behavior of the Asiatic elephant (Elephas maximus maximus L.). Behaviour 38:193-225.

Evans, K. E., and S. Harris. 2008. Adolescence in male African elephants, Loxodonta africana, and the importance of sociality. Anim Behav 76(3):779-787.

Ganswindt, A., M. Heistermann, and K. Hodges. 2005a. Physical, Physiological, and Behavioral Correlates of Musth in Captive African Elephants (Loxodonta africana). Physiological and Biochemical Zoology 78(4):505-514. doi: $10.1086 / 430237$

Ganswindt, A., S. Muenscher, M. Henley, S. Henley, M. Heistermann, R. Palme, P. Thompson, and H. Bertschinger. 2010. Endocrine correlates of musth and the impact of ecological and social factors in free-ranging African elephants (Loxodonta africana). Horm Behav 57(4):506-514. doi: https://doi.org/10.1016/j.yhbeh.2010.02.009

Ganswindt, A., R. Palme, M. Heistermann, S. Borragan, and J. Hodges. 2003. Noninvasive assessment of adrenocortical function in the male African elephant (Loxodonta africana) and its relation to musth. Gen Comp Endocrinol 134(2):156-166.

Ganswindt, A., H. B. Rasmussen, M. Heistermann, and J. K. Hodges. 2005b. The sexually active states of free-ranging male African elephants (Loxodonta africana): defining musth and non-musth using endocrinology, physical signals, and behavior. Horm Behav 47(1):83-91.

Ghosal, R., A. Ganswindt, P. B. Seshagiri, and R. Sukumar. 2013. Endocrine Correlates of Musth in Free-Ranging Asian Elephants (Elephas maximus) Determined by Non-Invasive Faecal Steroid Hormone Metabolite Measurements. PLoS One 8(12):e84787. doi: 10.1371/journal.pone.0084787 
Goldenberg, S. Z., S. de Silva, H. B. Rasmussen, I. Douglas-Hamilton, and G. Wittemyer. 2014. Controlling for behavioural state reveals social dynamics among male African elephants, Loxodonta africana. Anim. Behav 95:111-119. doi: https://doi.org/10.1016/j.anbehav.2014.07.002

Goymann, W. 2012. On the use of non-invasive hormone research in uncontrolled, natural environments: the problem with sex, diet, metabolic rate and the individual. Methods Ecol Evol 3(4):757-765. doi: 10.1111/j.2041210X.2012.00203.X

Greco, B. J., C. L. Meehan, J. N. Hogan, K. A. Leighty, J. Mellen, G. J. Mason, and J. A. Mench. 2016. The Days and Nights of Zoo Elephants: Using Epidemiology to Better Understand Stereotypic Behavior of African Elephants (Loxodonta africana) and Asian Elephants (Elephas maximus) in North American Zoos. PLoS One 11(7):e0144276. doi: 10.1371/journal.pone.0144276

Hall-Martin, A. J., and L. A. Van der Walt. 1984. Plasma testosterone levels in relation to musth in the male African Elephant. Koedoe 27(1):3. doi: 10.4102/koedoe.v27i1.561

Harrison, X. A., L. Donaldson, M. E. Correa-Cano, J. Evans, D. N. Fisher, C. E. D. Goodwin, B. S. Robinson, D. J. Hodgson, and R. Inger. 2018. A brief introduction to mixed effects modelling and multi-model inference in ecology. PeerJ 6:e4794. doi: $10.7717 /$ peerj.4794

Hartley, M., A. Wood, and L. Yon. 2019. Facilitating the social behaviour of bull elephants in zoos. 53(1):62-77. doi: 10.1111/izy.12245

Hawkley, L. C., S. W. Cole, J. P. Capitanio, G. J. Norman, and J. T. Cacioppo. 2012. Effects of social isolation on glucocorticoid regulation in social mammals. Horm Behav 62(3):314-323. doi: https://doi.org/10.1016/j.yhbeh.2012.05.011

Howland, B. E., L. M. Sanford, and W. M. Palmer. 1985. Changes in serum levels of LH, FSH, prolactin, testosterone, and cortisol associated with season and mating in male pygmy goats. J Androl 6(2):89-96. doi: 10.1002/j.19394640.1985.tb00822.x

Jainudeen, M. R., C. B. Katongole, and R. V. Short. 1972a. Plasma testosterone levels in relation to musth and sexual activity in the male Asiatic elephant, Elephas maximus. J Reprod Fertil 29:99-103.

Jainudeen, M. R., G. M. McKay, and J. F. Eisenberg. 1972b. Observations on musth in the domesticated Asiatic elephant (Elephas maximus). Mammalia 36:247-261. 
Kajaysri, J., and W. Nokkaew. 2014. Assessment of pregnancy status of Asian elephants (Elephas maximus) by measurement of progestagen and glucocorticoid and their metabolite concentrations in serum and feces, using enzyme immunoassay (EIA). J Vet Med Sci 76(3):363-368. doi: https://doi.org/10.1292/jvms.13-0103

Keerthipriya, P., S. Nandini, and T. N. C. Vidya. 2018. Effects of male age and female presence on male associations in a large, polygynous mammal in southern India. bioRxiv:485144. doi: 10.1101/485144 \%J bioRxiv

Keerthipriya, P., and T. N. C. Vidya. 2019. A Comparison of the Occurrence of Musth in the Kabini Elephant Population with Other Populations. Gajah 50:4-16.

Khonmee, J., J. L. Brown, S. Rojanasthien, D. Thumasanukul, A. Kongphoemphun, B. Siriaroonrat, W. Tipkantha, V. Punyapornwithaya, and C. Thitaram. 2014. Seasonality of fecal androgen and glucocorticoid metabolite excretion in male goral (Naemorhedus griseus) in Thailand. Anim Reprod Sci 146(1):70-78. doi: https://doi.org/10.1016/j.anireprosci.2014.02.008

Kumar, V., V. Palugulla Reddy, A. Kokkiligadda, S. Shivaji, and G. Umapathy. 2014. Non-invasive assessment of reproductive status and stress in captive Asian elephants in three south Indian zoos. Gen Comp Endocrinol 201:37-44. doi: https://doi.org/10.1016/j.ygcen.2014.03.024

LaDue, C., N. Scott, and S. Margulis. 2014. A survey of musth among captive male elephants in North America: updated results and implications for management. Journal of the Elephant Managers Association (JEMA) 25(2):18-24.

Lee, B. 2019. AZA Regional Studbook for Asian Elephant (Elephas maximus). Association of Zoos and Aquariums, Portland, OR.

Lincoln, G. A., and W. D. Ratnasooriya. 1996. Testosterone secretion, musth behaviour and social dominance in captive male Asian elephants living near the equator. Reproduction 108(1):107. doi: 10.1530/jrf.0.1080107

Liptrap, R., and J. Raeside. 1968. Effect of corticotrophin and corticosteroids on plasma interstitial cell-stimulating hormone and urinary steroids in the boar. J Endocrinol 42(1):33-43.

Liptrap, R. M., and J. I. Raeside. 1978. A relationship between plasma concentrations of testosterone and corticosteroids during sexual and aggressive behaviour in the boar. J Endocrinol 76(1):75-85. doi: 10.1677/joe.0.0760075 
McEwen, B. S., and J. C. Wingfield. 2003. The concept of allostasis in biology and biomedicine. Horm Behav 43(1):2-15. doi: https://doi.org/10.1016/S0018506X(02)00024-7

Menargues, A., V. Urios, and M. Mauri. 2008. Welfare assessment of captive Asian elephants (Elephas maximus) and Indian rhinoceros (Rhinoceros unicornis) using salivary cortisol measurement. Anim Welf 17:305-312.

Metrione, L. C., D. Hunter, and L. M. Penfold. 2020. Seasonal Changes in Fecal Glucocorticoid Metabolite Concentrations in Bison (Bison bison) Living with or without Wolves (Canis lupus). Journal of Wildlife Diseases 56(1):175-178. doi: $10.7589 / 2017-12-308$

Miller, M. A., M. Finnegan, T. Storms, M. Garner, and K. P. Lyashchenko. 2018. Outbreak of Mycobacterium Tuberculosis in a Herd of Captive Asian Elephants (Elephas maximus): Antemortem Diagnosis, Treatement, and Lessons Learned. J Zoo Wildl Med 49(3\%J Journal of Zoo and Wildlife Medicine):748-754, 747.

Mooring, M. S., M. L. Patton, V. A. Lance, B. M. Hall, E. W. Schaad, G. A. Fetter, S. S. Fortin, and K. M. McPeak. 2006. Glucocorticoids of bison bulls in relation to social status. Hormones and Behavior 49(3):369-375. doi: 10.1016/j.yhbeh.2005.08.008

Morley, J. E., F. E. Kaiser, H. M. Perry, P. Patrick, P. M. K. Morley, P. M. Stauber, B. Vellas, R. N. Baumgartner, and P. J. Garry. 1997. Longitudinal changes in testosterone, luteinizing hormone, and follicle-stimulating hormone in healthy older men. Metabolism 46(4):410-413. doi: https://doi.org/10.1016/S00260495(97)90057-3

Möstl, E., and R. Palme. 2002. Hormones as indicators of stress. Domestic Animal Endocrinology 23(1):67-74. doi: https://doi.org/10.1016/S0739-7240(02)00146-7

Muller, M. N., and R. W. Wrangham. 2004. Dominance, cortisol and stress in wild chimpanzees (Pan troglodytes schweinfurthii). Behav. Ecol. Sociobiol. 55(4):332340. doi: 10.1007/s00265-003-0713-1

Niemuller, C. A., and R. M. Liptrap. 1991. Altered androstenedione to testosterone ratios and LH concentrations during musth in the captive male Asian elephant (Elephas maximus). J Reprod Fertil 91(1):139-146. doi: 10.1530/jrf.0.0910139 
Palme, R. 2019. Non-invasive measurement of glucocorticoids: Advances and problems. Physiol Behav 199:229-243. doi: https://doi.org/10.1016/j.physbeh.2018.11.021

Pérez-Rodríguez, L., J. Blas, J. Viñuela, T. A. Marchant, and G. R. Bortolotti. 2006. Condition and androgen levels: are condition-dependent and testosteronemediated traits two sides of the same coin? Anim Behav 72(1):97-103. doi: https://doi.org/10.1016/j.anbehav.2005.09.021

Poole, J. H. 1987. Rutting behavior in African elephants: the phenomenon of musth. Behaviour 102:283-316.

Poole, J. H. 1989a. Announcing intent: the aggressive state of musth in African elephants. Anim Behav 37:140-152.

Poole, J. H. 1989b. Mate guarding, reproductive success and female choice in African elephants. Anim Behav 37:842-849.

Poole, J. H., and C. J. Moss. 1981. Musth in the African elephant, Loxodonta africana. Nature 292:830-831.

Prado-Oviedo, N. A., M. K. Bonaparte-Saller, E. J. Malloy, C. L. Meehan, J. A. Mench, K. Carlstead, and J. L. Brown. 2016. Evaluation of Demographics and Social Life Events of Asian (Elephas maximus) and African Elephants (Loxodonta africana) in North American Zoos. PLoS One 11(7):e0154750. doi: https://doi.org/10.1371/journal.pone.0154750

R Core Team. 2020. R: A language and environment for statistical computing. $\mathrm{R}$ Foundation for Statistical Computing; Available from: https://www.r-project.org/, Vienna, Austria.

Ralph, C. R., and A. J. Tilbrook. 2016. INVITED REVIEW: The usefulness of measuring glucocorticoids for assessing animal welfare. J Anim Sci 94(2):457-470. doi: https://doi.org/10.2527/jas.2015-9645

Rasbash, J., C. Charlton, W. Browne, M. Healy, and B. Cameron. 2005. MLwiN v 2.02. University of Bristol: Centre for Multilevel Modelling; Available from: http://www.bristol.ac.uk/cmm/software/mlwin/, Bristol, United Kingdom.

Rasmussen, H. B., A. Ganswindt, I. Douglas-Hamilton, and F. Vollrath. 2008. Endocrine and behavioral changes in male African elephants: linking hormone changes to sexual state and reproductive tactics. Horm Behav 54(4):539-548. 
Rasmussen, L. E., I. O. Buss, D. L. Hess, and M. J. Schmidt. 1984. Testosterone and dihydrotestosterone concentrations in elephant serum and temporal gland secretions. Biol Reprod 30(2):352-362.

Rasmussen, L. E., H. S. Riddle, and V. Krishnamurthy. 2002. Mellifluous matures to malodorous in musth. Nature 415(6875):975-976. doi: 10.1038/415975a

Rasmussen, L. E. L., D. L. Hess, and J. D. Haight. 1990. Chemical analysis of temporal gland secretions collected from an Asian bull elephant during a four-month musth episode. Journal of Chemical Ecology 16(7):2167-2181. (journal article) doi: $10.1007 / \mathrm{bf} 01026928$

Rasmussen, L. E. L., and T. E. Perrin. 1999. Physiological Correlates of Musth: Lipid Metabolites and Chemical Composition of Exudates. Physiology \& Behavior 67(4):539-549. doi: https://doi.org/10.1016/S0031-9384(99)00114-6

Rosen, L. E., F. Olea-Popelka, S. L. Deem, R. Isaza, D. Schmitt, and M. Miller. 2019. Survey of Antituberculosis Drug Administration and Adverse Effects in Elephants in North America. J Zoo Wildl Med 50(1):23-32. doi: 10.1638/2018-0028

Samaras, N., D. Samaras, E. Frangos, A. Forster, and J. Philippe. 2013. A review of agerelated dehydroepiandrosterone decline and its association with well-known geriatric syndromes: is treatment beneficial? Rejuvenation research 16(4):285294.

Sands, J., and S. Creel. 2004. Social dominance, aggression and faecal glucocorticoid levels in a wild population of wolves, Canis lupus. Anim. Behav 67(3):387-396. doi: https://doi.org/10.1016/j.anbehav.2003.03.019

Scallan, J., S. Glaeser, K. Backues, and E. Freeman. 2011. Serum Testosterone Concentrations in an ex situ male Asian Elephant (Elephas maximus) in relation to a temporary breeding loan. Journal of the Elephant Managers Association (JEMA) 22(3):20-33.

Schmid, J., M. Heistermann, U. Gansloßer, and J. Hodges. 2001. Introduction of foreign female Asian elephants (Elephas maximus) into an existing group: behavioural reactions and changes in cortisol levels. Anim Welf 10(4):357-372.

Schulte, B. A., and L. E. L. Rasmussen. 1999. Musth, Sexual Selection, Testosterone, and Metabolites. In: R. E. Johnston, D. Müller-Schwarze and P. W. Sorensen, editors, Advances in Chemical Signals in Vertebrates. Springer US, Boston, MA. p. 383397. 
Scott, N. L. 2002. Chemical communication and musth in captive male elephants., Portland, OR (USA).

Scott, N. L., and H. Riddle. 2003. Assessment of Musth in Captivity: A Survey of Factors Affecting the Frequency and Duration of Musth in Captive Male Elephants Elephas maximus - Loxodonta africana. Journal of the Elephant Managers Association (JEMA) 14(2):11-15.

Somgird, C., S. Sripiboon, S. Mahasawangkul, K. Boonprasert, J. L. Brown, T. A. Stout, B. Colenbrander, and C. Thitaram. 2016. Differential testosterone response to GnRH-induced LH release before and after musth in adult Asian elephant (Elephas maximus) bulls. Theriogenology 85(7):1225-1232.

Thongtip, N., J. Saikhun, S. Mahasawangkul, K. Kornkaewrat, P. Pongsopavijitr, N. Songsasen, and A. Pinyopummin. 2008. Potential factors affecting semen quality in the Asian elephant (Elephas maximus). Reproductive Biology and Endocrinology 6(1):9. doi: 10.1186/1477-7827-6-9

Vidya, T. N. C., and R. Sukumar. 2005. Social and reproductive behaviour in elephants. Current Science 89(7):1200-1207.

Wang, C., A. Leung, and A. P. Sinha-Hikim. 1993. Reproductive aging in the male brown-Norway rat: a model for the human. Endocrinology 133(6):2773-2781. doi: 10.1210/endo.133.6.8243304 \%J Endocrinology

Yon, L., S. Kanchanapangka, N. Chaiyabutr, S. Meepan, F. Z. Stanczyk, N. Dahl, and B. Lasley. 2007. A longitudinal study of LH, gonadal and adrenal steroids in four intact Asian bull elephants (Elephas maximus) and one castrate African bull (Loxodonta africana) during musth and non-musth periods. General and Comparative Endocrinology 151(3):241-245. doi: https://doi.org/10.1016/j.ygcen.2007.01.028 
CHAPTER 4: Supporting Asian elephant (Elephas maximus) welfare and herd dynamics with a more complex and expanded habitat at the Oregon Zoo

Sharon S. Glaeser ${ }^{1, *}$, David Shepherdson ${ }^{1}$, Karen Lewis ${ }^{1}$, Natalia Prado ${ }^{2}$, Janine L. Brown $^{2}$, Nadja Wielebnowski ${ }^{1}$

1 Oregon Zoo, 4001 SW Canyon Rd, Portland, Oregon, 97221, United States of America, 2 Center for Species Survival, Smithsonian Conservation Biology Institute, Smithsonian National Zoological Park, Front Royal, Virginia, United States of America

* Sharon.Glaeser@oregonzoo.org

Keywords: welfare, behavior, locomotion, GPS, glucocorticoids, adrenal activity, enclosure use, post-occupancy, feeding predictability, zoo, elephant 


\section{Abstract}

The Oregon Zoo took a welfare-based approach in the design of a new habitat for their Asian elephants, with a focus on meeting the elephants' physiological, psychological, and social needs 24 hours a day and across life stages. The habitat was designed to encourage activity, promote species-typical behaviors, offer increased opportunities for choice and social interaction, and provide biologically meaningful challenges. A four-year study was conducted to monitor welfare throughout the transition and acclimation from the previous habitat to the new habitat, and to evaluate the effectiveness of the habitat at achieving program goals. Welfare indicators included reproductive and adrenal hormone analyses, detailed longitudinal behavior assessments and distance walked through GPS monitoring. Results show that the Oregon Zoo has achieved its goals with the new habitat. The elephants were more active and walked farther on a daily basis in the new habitat, with an average walking distance of over $15 \mathrm{~km}$ per day. Repetitive behaviors occurred at a low rate and only in two females, decreasing in the new habitat in one female, and with a change in bout pattern in the other female. A switch from primarily keeper-delivered food to seeking food on their own indicates that the disbursement of food with less temporal and spatial predictability provided increased foraging opportunities, which help to satisfy appetitive motivations important for psychological well-being. The elephants were also able to make more choices of their own volition in their new environment, with larger spaces and indoor and outdoor access available most of the time, and they regularly used the entire habitat without avoiding specific areas. All individuals showed adaptive and normal adrenal responses to change and challenge, with the highest fecal glucocorticoid 
metabolite (FGM) concentrations and variability during the construction phase, and a return to previous baseline concentrations in the new habitat, suggesting they adapted well to the changes. The elephants expressed a diverse range of species-typical behaviors and demonstrated social dynamics of a healthy herd in both the old and new habitats. The percentage of time spent in proximity of other elephants decreased for all individuals (average $25 \%$ decrease), but time spent engaging in active social behaviors increased, or was similar between habitats for all elephants except a young male, suggesting more choice in whether to spend time together or move away to engage in other activities. Elephants made different choices between the habitats in who to spend time with, which reflects natural and changing herd dynamics with a young male and female calf transitioning through life stages. Findings indicate that the complexity and flexibility of the new habitat and habitat management has been effective in improving overall welfare by providing meaningful challenges and the opportunity to express appetitive behaviors, by offering choice in environmental conditions, and by providing the space and resource distribution to support increased social equity for individuals and changing herd dynamics. 


\section{Introduction}

As zoos more extensively use evidence-based approaches to manage animals for improved welfare, there is an increasing need to identify environmental factors that contribute to welfare outcomes. The Oregon Zoo took a welfare-based approach in the design of its new elephant habitat — an approach focused on meeting the elephants' physiological, psychological, and social needs 24 hours a day and across their life stages. The new habitat was designed to encourage activity, promote species-typical behaviors, offer increased opportunities for choice, and provide biologically meaningful challenges. Design decisions were informed by decades of research, hands-on experience, and an understanding of elephants' individual needs; and were ultimately supported by outcomes from the largest zoo-based elephant welfare study to date (Meehan et al., 2016), which found a variety of social, housing, and management factors associated with multiple indicators of health and welfare in both Asian and African elephants (Brown et al., 2016; Greco et al., 2016; Miller et al., 2016; Morfeld et al., 2016).

To promote a diverse range of species-typical behaviors, the habitat needed to support natural herd dynamics with the ability to live in multi-generational matrilineal groups, which bulls can join or leave as they would in free-ranging populations (McKay, 1973) and the opportunity for young males to learn from older bulls (Sukumar, 2003; Rees, 2004; Garai and Kurt, 2006; Evans and Harris, 2008). It required a flexible space with indoor-outdoor access, an enriched environment with a variety of features to engage with, and an increased ability for elephants to choose where and how they spend their time and 
who they spend it with. The importance of providing zoo animals with appropriate challenges (Meehan and Mench, 2007; Clark, 2017) and the related variables of choice and control (Owen et al., 2005; Buchanan-Smith and Badihi, 2012) through environmental enrichment (Shepherdson et al., 1993; Shepherdson et al., 1998; Wagman et al., 2018) has been convincingly documented for a wide variety of species, including elephants (Shepherdson, 1999).

The habitat was designed to allow for flexible feeding strategies that encourage foraging and exploration, with unpredictability of food disbursement across time and space to fulfil the occupational need to forage, and with resources requiring elephants to work for food to increase feeding time. In the wild, food resources are temporally and spatially dispersed, and elephants use a range of foraging skills to locate and access food (Spinage, 1994; Sukumar, 2003). Studies have shown that provision of food to animals in human care in a form that requires species-specific food-finding behavior promotes exploratory behavior and reduces levels of stereotypic behaviors [reviewed in Shepherdson et al. (1993)]. Varying spatial predictability is analogous to feeding in the wild, with the degree of predictability depending on the feeding ecology of the species (Bassett and BuchananSmith, 2007). Feeding diversity and unpredictable feeding schedules have been associated with increased walking distance in both African and Asian zoo-housed elephants (Holdgate et al., 2016). In the new habitat, a diversity of food delivery methods provides foraging opportunities for 14 to 16 hours per day including crepuscular and nocturnal periods, which more closely mimics the foraging habits of free-ranging elephants (Spinage, 1994; Sukumar, 2003) and supports the 24/7 approach to animal 
welfare (Brando and Buchanan-Smith, 2018). Throughout the habitat, timed feeders release food at programmable intervals, overhead feeders require elephants to stretch and sometimes climb on logs, herd feeders require reaching down, and other puzzle feeders demand manipulation to acquire food. The timed feeders dispersed throughout the habitat present a dynamic feeding regime whereby elephants can find food as a consequence of exploring their habitat. The habitat shape and topographical complexity was designed to encourage exploration and activity; the elephants cannot see the entire space from any one vantage point and get exercise simply by manoeuvring through it. The hilly terrain, climbing features, and varied surfaces - including deep sand and hills of dirt — provide stimulation and physical challenges.

A four-year study was conducted to evaluate the effectiveness of the new habitat in achieving elephant care goals through assessment of welfare indicators before, during and after transition to the new habitat. Whitham and Wielebnowski (2013) recommended integrating behavioral, physiological, and biological measures of good well-being, as each measure alone can be limited in its usefulness in interpretation. Welfare indicators included distance walked through GPS monitoring, reproductive and adrenal hormone analyses, and detailed behavior assessments. Data collection began while the elephants still occupied their previous habitat, and continued through construction and one year after the opening of the new habitat called "Elephant Lands".

Walking distance and patterns in wild elephants are affected by a variety of factors, including the distribution and availability of resources, season, age, sex, reproductive 
state, presence of calves or juveniles in a group, social group ranks, and human activities (McKay, 1973; Theuerkauf and Ellenberg, 2000; Whitehouse and Schoeman, 2003; Slotow and van Dyk, 2004; Wittemyer et al., 2007; Leighty et al., 2009; Boult et al., 2019; Sach et al., 2019). The relationship between resource availability and distances travelled suggests that walking varies in response to external conditions. Under professional care, food and water resources are provided so the need for walking is reduced. However, walking also supports exploratory behavior, which has an information-gathering function and may be rewarding even when not for the purpose of resource acquisition (Shepherdson et al., 1993; Mench, 1998; Boissy et al., 2007). In addition, there is evidence that daily exercise in general improves animal body condition (Morfeld et al., 2016) and foot health (Lewis et al., 2010). By comparing the distance walked and patterns of space use in different habitats, we can better understand the social, environmental, and management-related factors influencing movement, and evaluate walking distance in association with other indicators of welfare.

Hormone measurements can provide information on reproductive state and adrenal activity, which helps to assess the physiological aspect of animal health and well-being (Wielebnowski et al., 2002a; Wielebnowski et al., 2002b; Wielebnowski, 2003). In our previous long-term study of gonadal (Glaeser et al., 2012) and adrenal activity (Glaeser et al., in review) of female Asian elephants, we found that major life events — births, deaths, changes in herd structure — had minimal effect on estrous-cycle dynamics over time and adrenal responses were activated only short-term, suggesting that the Oregon Zoo's female elephants are quite resilient and maintained normal reproductive and 
adrenal function throughout all the changes. Indicators of stress (i.e., distress and chronic stress) are commonly measured through welfare outcomes, with the most frequently used indicator being glucocorticoids (e.g., cortisol, corticosterone) secreted from the adrenal cortex in response to a variety of stimuli (Möstl and Palme, 2002; Buwalda et al., 2012; Ralph and Tilbrook, 2016; Palme, 2019). When there is a major change constituting a real or perceived stressor, we see an adaptive physiological response that includes resilience, whereby glucocorticoids secreted from the adrenal cortex in response to the stressor are temporarily elevated and subsequently decrease back to a baseline in an individual. Through hormone monitoring in this study we wanted to make sure that individuals could deal with challenges of construction and transition to new habitats in a normal and adaptive manner, and that their responses would not have any long-term negative effects, but rather beneficial effects of arousal and additional opportunities to exercise cognitive and behavioral skills (Meehan and Mench, 2007). Because the stimuli that activate the stress response can be positive or negative, it is important to measure additional indicators of welfare.

Behavior measurements can provide information on activity level, social relationships, habitat utilization and resource use, and the choices animals make without intervention by animal-care staff. The presence of an appropriate frequency and diversity of speciestypical behaviors is a potential indicator that the needs of an animal are being met and that it is experiencing good health and well-being (McPhee and Carlstead, 2010). By monitoring behavior of groups or individuals across different conditions we can identify factors associated with behavioral change as well as behavioral patterns that are persistent 
(Watters et al., 2009). Individuals respond differently to the same stimuli, and a facility transition for this group of elephants was expected to present many new opportunities and meaningful challenges. Time budgets can provide a baseline against which to assess the effect of changes. Social interactions tell us about relationships and choices given social opportunities. Engagement with resources informs us on how various aspects of the physical environment support self-maintenance and provide opportunities to overcome appropriate challenges, make choices, and allow individuals some level of control over their environment (reviewed in Whitham and Wielebnowski (2013)).

By analyzing quantitative behavioral and physiological data on all of the elephants, this study aimed to evaluate their health and well-being throughout the transition period, as well as assess the effectiveness of design features and the associated changes in management practices in achieving program goals. By evaluating welfare outcomes on individual elephants under multiple environmental conditions, we were able to test the direct influence of various environmental factors on welfare. The outcomes of this study provide detailed data for comparison and offer insight on habitat features that can help in meeting the physiological, psychological, and social needs of elephants under human care. In addition, the assessment methods used to quantify the effect of habitat and social changes could also provide a model for future investigations of individual response to various transitions — facility transfers, habitat modifications, changes in social structure, rehabilitation and release, translocations — as well as for long-term welfare monitoring. 


\section{Materials and Methods}

$\underline{\text { Animals }}$

Asian elephants $(\mathrm{n}=5)$ were housed at the Oregon Zoo (females: $\mathrm{n}=3$ Elephas maximus indicus, $\mathrm{n}=1$ E. $m$. borneensis, males: $\mathrm{n}=1$ E. $m$. indicus) (Table 1). Three adult bulls were also housed at the zoo, but were not included in this analysis because two individuals were humanely euthanized prior to full occupancy of the new habitat and the third individual was undergoing medical treatment that prohibited full occupancy. This study was approved by the Welfare and Research Review Committees of the Oregon Zoo.

Table 1: Elephants included in this study

\begin{tabular}{|c|c|c|c|c|}
\hline Elephant & Sex & Origin & $\begin{array}{c}\text { Date of birth or } \\
\text { transfer to Oregon Zoo }\end{array}$ & $\begin{array}{c}\text { Age at start of } \\
\text { sample collection }\end{array}$ \\
\hline Female1 & Female & $\begin{array}{c}\text { Zoo-born } \\
\text { Oregon Zoo }\end{array}$ & 26 Dec 1982-Birth & 29 yrs. \\
\hline Female2 & Female & $\begin{array}{c}\text { Wild } \\
\text { Borneo, Malaysia }\end{array}$ & $\begin{array}{c}\sim 1993-\text { Birth } \\
20 \text { Nov 1999 - Transfer } \\
\text { to Oregon Zoo }\end{array}$ & 19 yrs. \\
\hline Female3 & Female & $\begin{array}{c}\text { Zoo-born } \\
\text { Oregon Zoo }\end{array}$ & 31 Aug 1994-Birth & 18 yrs. \\
\hline M-juvenile & Male & $\begin{array}{c}\text { Zoo-born } \\
\text { Oregon Zoo }\end{array}$ & 23 Aug 2008-Birth & 4 yrs. \\
\hline F-calf & Female & $\begin{array}{c}\text { Zoo-born } \\
\text { Oregon Zoo }\end{array}$ & 30 Nov 2012 - Birth & 2 mo. \\
\hline
\end{tabular}




\section{$\underline{\text { Study time period and habitats }}$}

The timeline (Fig. 1) comprised three habitat phases: occupancy in the previous habitat; period of change with construction, introduction to new space (4 new areas), reconfigurations of familiar spaces, and a move to the new building; and occupancy in the new habitat. Sample collection began in September 2012 while the elephants still occupied their previous habitat, and continued through the end of 2016, one year after the full opening of the new habitat. Construction began on 04Apr2013 and ended on 12Dec2015. Elephants were introduced to new habitat areas with new resources on 22Feb2014, 19May2015, 04Jun2015, and 12Dec2015.

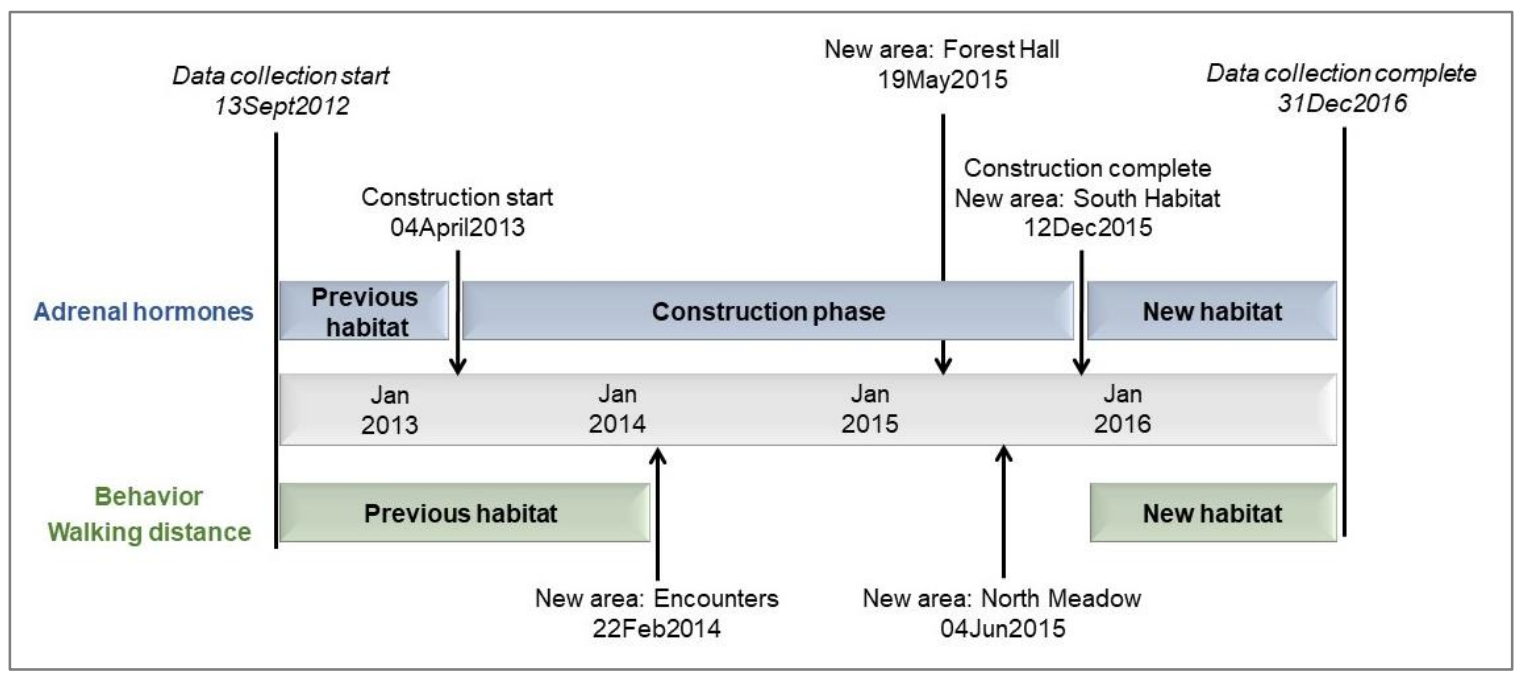

Fig 1: Study timeline

For analysis of behavior and walking distance, the first introduction to a new yard on 22Feb2014 marked the end of the "previous habitat" phase, and the first day with full access to the new habitat on 12Dec2015 marked the start of the "new habitat" phase, resulting in durations of 75 weeks in the previous habitat and 55 weeks in the new habitat. For analysis of adrenal hormones, the habitat phases were defined by 
construction start and end dates in order to evaluate response to construction activities, resulting in durations for analysis of 29 weeks in the previous habitat, 140 weeks during the period of change, and 55 weeks in the new habitat.

The previous habitat consisted of two oval-shaped outside yards on either side of the elephant building, both with sand substrate. The smaller yard was $1,050 \mathrm{~m}^{2}\left(11,305 \mathrm{ft}^{2}\right)$, with a pool and shade structure. The larger yard was $2,350 \mathrm{~m}^{2}\left(25,300 \mathrm{ft}^{2}\right)$, with a 302,800-litre (80,000-gallon) pool surrounded by a concrete apron, a shade structure, a scratching wall mid-yard with hardware for attaching enrichment objects, a vertical log for hanging enrichment, a large water drink tank, and one timed feeder. The inside area consisted of seven temperature-controlled rooms with rubber flooring, each with a water tank and several structures that provided options for enrichment.

The new habitat is approximately four times the size of the previous habitat, with a total habitat area of $18,210 \mathrm{~m}^{2}$ (1.82 ha/4.5 acres). Three connected outdoor yards have varied terrain and a perimeter of a $2.1 \mathrm{~km}(1.3 \mathrm{mi})$. Indoor spaces total nearly $3,065 \mathrm{~m}^{2}(33,000$ $\left.\mathrm{ft}^{2}\right)$. All elephant habitat areas are covered with a sand substrate and also with dirt in some areas outside. Outdoor and indoor spaces are linked with multiple options for configuring the space for individuals and groups. The indoor space is climate controlled, and outdoor shade structures are installed with overhead heaters and misters. Feeding features include timed feeders throughout the habitat, various overhead feeders (nets, barrels, puzzles), and partially buried herd feeders. Water features include a 605,600-litre

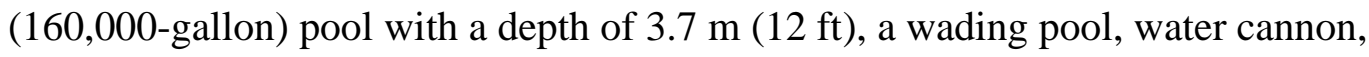


drinkers throughout the habitat, and a deluge sprinkler system indoors to simulate rain and also for deep cleaning of the sand substrate. Other features include dirt hills and mud wallows, large logs and rocks for climbing, and structures such as vertical logs that provide options for keepers to attach non-permanent environment enrichment objects.

\section{$\underline{\text { Data analysis }}$}

Statistical analyses are described in the methods section for each welfare indicator. Descriptive statistics and charting were performed using Excel 2016. Statistical analyses were conducted in R version 3.6.3 for Windows (R Core Team, 2020). Significance was assessed at the $\mathrm{p}=0.05$ level.

\section{Walking distance and space use}

In 2012, scientists measured outdoor walking distance in 56 adult female African and Asian elephants, 12 years and older, in 30 North American AZA zoos (Holdgate et al., 2016) as part of a larger multi-institutional elephant welfare study (Meehan et al., 2016). The Oregon Zoo's elephants were part of this study, and therefore we were able to use these existing data as the measurement of outdoor daily walking distance in the previous habitat. We then applied the already proven methodology for our current study to compare distance walked between the new habitat and previous habitats.

Bracelets equipped with GPS data loggers were worn by the same two adult females as in the 2012 study (Female1 and Female2) plus two males (M-juvenile and an adult bull). The third adult female, Female3, was not measured for distance walked as we expected 
her movement to be influenced by her calf (Kurt, 1974). Bracelets were worn for 24-hour periods, approximately every two weeks, from June 2014 through December 2016. All elephants were acclimated to wearing the bracelets through desensitization using positive reinforcement. Nevertheless, GPS monitoring days did not coincide with behavior monitoring days to avoid any influence the GPS anklet might have had on behavior. GPS units were programmed to record data at five-second intervals. Each data point included the date, time, latitude, longitude, and two indices of estimate quality (number and geometry of satellites used) in each location (Holdgate et al., 2016). GPS data is unreliable when communication between satellites and receivers is obstructed, for example under a dense forest canopy or inside a building. Data points known to have occurred while the elephants were indoors were therefore removed. In ArcMap software, habitat boundaries were defined using Google Earth imagery (Fig. 2). GPS data was then mapped onto the habitat and the clip function was used to remove any remaining data from indoor areas and any data that fell outside of the boundaries. Clipped data was exported to Excel, then data points that failed to meet location estimate quality criteria were removed. The Euclidean (straight line) distance between consecutive data points was calculated, then screened for distances greater than an elephant can travel using a near maximal velocity of $6.8 \mathrm{~m} / \mathrm{s}$ measured for elephants (Hutchinson et al., 2006). The final straight-line distance between consecutive data points was then summed to obtain total distances travelled for each day.

Holdgate et al. (2016) collected GPS data for five days within a month that minimized inter-zoo variation in predicted daily temperature (late August/early September for the 
Oregon Zoo). Walking distance was measured only for elephants that had outdoor access for at least 20 of the 24 hours for at least three days. For comparison of distance walked in the new habitat to the previous habitat, a subset of the data was used that met the same criteria of 20 hours and the same season (July through September) rather than limiting data collection to the same month. In this timeframe, Female1 had six days that met these criteria, and Female2 had seven days. Mann-Whitney U-tests were used to compare mean daily walking distance between the new and previous habitats.

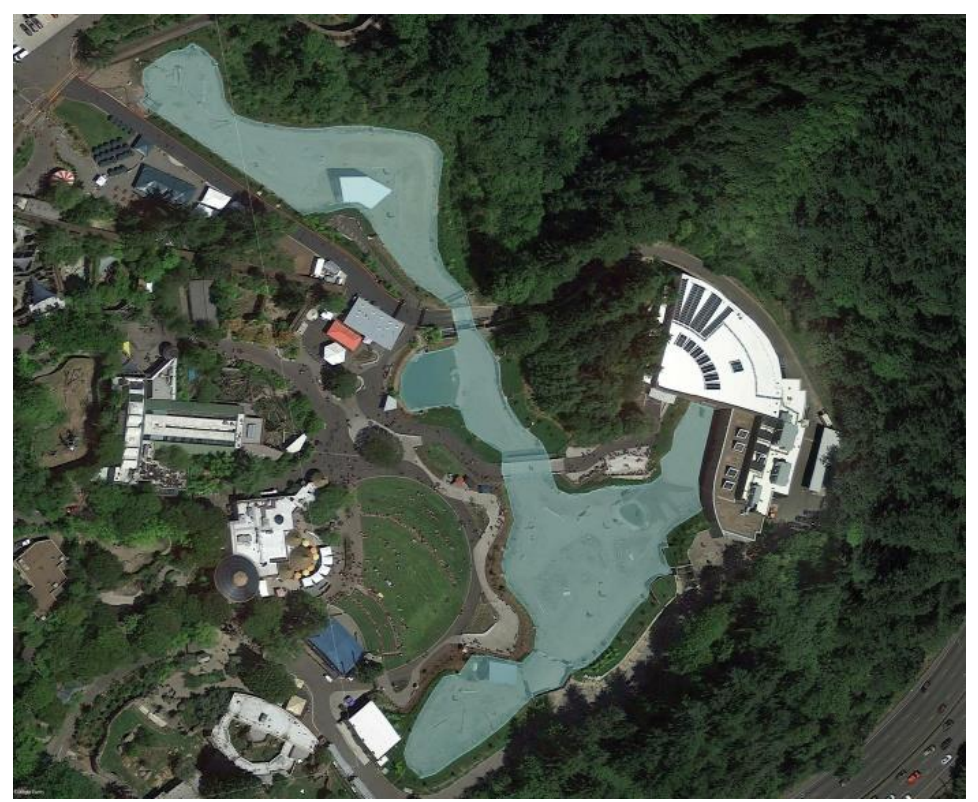

Fig 2: New habitat with outdoor area shaded using ArcMap

\section{$\underline{\text { Reproductive and adrenal hormones }}$}

Adrenal and reproductive hormones were analyzed to assess adrenal activity and reproductive cycling throughout the study period, from September 2012 through December 2016. Fecal and blood samples were collected weekly from all individuals, and 
were collected in the morning to control for any diurnal patterns of hormone secretion and excretion (Brown et al., 2010).

Progestagens and testosterone in serum are routinely measured in Oregon Zoo elephants to determine ovarian cyclicity in females and musth correlates in males. All elephants in this study were trained for voluntary blood collection (without sedation) as part of their normal management routine. Blood was collected (3-9 $\mathrm{mL})$ into red top serum separator tubes from an ear or leg vein by elephant care staff. Blood was maintained at $\sim 4{ }^{\circ} \mathrm{C}$, then centrifuged at $1500 \mathrm{~g}$ within a few hours of collection to separate serum, and stored at $-20^{\circ} \mathrm{C}$ until analysis. Serum samples were analyzed without extraction. Progestagens and testosterone in serum samples collected through 2014 were measured in Oregon Zoo's endocrine lab using solid-phase ${ }^{125}$ I radioimmunoassays (RIA) (Siemens Healthcare Diagnostics Inc.) previously validated in elephants (progesterone (Brown and Lehnhardt, 1995; Brown et al., 2004; Brown, 2014); testosterone (Brown et al., 1993; Brown et al., 2007)), and modified with additional low standards for increased sensitivity. After the production of these RIA assays was discontinued in 2014 making them no longer commercially available, serum samples from 2015 through 2016 were analyzed using double-antibody enzyme immunoassays (EIA) validated for Asian elephants (progesterone: Oregon Zoo, unpublished; testosterone: Chapter 3). Assay sensitivities were $0.05 \mathrm{ng} / \mathrm{ml}$ for the progesterone RIA, $0.033 \mathrm{ng} / \mathrm{ml}$ for the progesterone EIA, 0.10 $\mathrm{ng} / \mathrm{ml}$ for the testosterone RIA, and $0.015 \mathrm{ng} / \mathrm{ml}$ for the testosterone EIA. 
Glucocorticoid metabolites excreted in feces (fecal glucocorticoid metabolites, FGM) were measured in samples collected with defecation times between 7a.m. and 11 a.m. in order to minimize possible diurnal fluctuation in FGM concentrations. Samples were collected within 2 hours of defecation, and were collected only for individuals observed defecating to ensure positive identification. Fecal matter was collected from the center of the bolus(es), then placed in zip-lock bags and stored at $-20^{\circ} \mathrm{C}$ until analysis. Fecal samples were shipped on dry ice overnight to the Smithsonian's Conservation Biological Institute endocrinology lab (Front Royal, VA) for analysis. Fecal samples were extracted using a dry-weight shaking extraction technique adopted from Scarlata et al. (2011) and modified by (Brown et al., 2019), with slight differences in centrifugation and sonication for this study. In brief, $0.1 \mathrm{~g}( \pm 0.01 \mathrm{~g})$ of lyophilized fecal powder was placed into $16 \mathrm{x}$ $125 \mathrm{~mm}$ glass tubes. Five $\mathrm{ml}$ of $80 \%$ methanol was added, and the samples were vortexed then agitated on a multi-tube pulse vortexer for $30 \mathrm{~min}$, followed by centrifugation at $2200 \mathrm{x}$ g for $20 \mathrm{~min}$. Supernatants were decanted into a second tube, and the remaining pellet was re-suspended in $5 \mathrm{ml}$ of $80 \%$ methanol and extracted again. The two supernatants were combined, and evaporated to dryness under forced air in a fume hood overnight. Dried extracts were reconstituted in $1 \mathrm{ml}$ of $100 \%$ methanol, and the tubes vortexed, then sonicated for $15 \mathrm{~min}$, then dried again. Final extracts were re-constituted in buffer ( $1 \mathrm{ml}, 0.149 \mathrm{M} \mathrm{NaCl}, 0.1 \mathrm{MNaPO}_{4}$; with $\left.\mathrm{pH} 7.0\right)$, then tubes sonicated for 30 seconds or longer to dissolve particulates. Finally, neat extracts were stored at $-20^{\circ} \mathrm{C}$ until analysis. The average extraction efficiency of this process was $85.7 \%$ (range $70.8-$ 99.5\%) based on addition of ${ }^{3} \mathrm{H}$-corticosterone (tracer) to each sample prior to extraction. 
FGM concentrations were determined using a double-antibody enzyme EIA with a polyclonal rabbit anti-corticosterone antibody (CJM006) validated for elephants by Watson et al. (2013). Standards (3.9-1000 pg/well; Sigma Diagnostics, St. Louis, MO), controls, and samples (diluted 1:10 or 1:20 (Female3 only) in assay buffer (Cat. No. X065, Arbor Assays, Arbor, MI, USA)) were added in duplicate (50 $\mu$ l per well) to precoated goat anti-rabbit IgG, 96-well plates at room temperature. Corticosteronehorseradish peroxidase $(25 \mu \mathrm{l} ; 1: 25,000)$ was added to all wells, followed by the primary anti-corticosterone antibody $(25 \mu 1 ; 1: 60,000)$ added to all except the non-specific binding wells. The plates were covered with microplate sealers and incubated at room temperature on an agitator (Model E6121; Eberbach Corp., Ann Arbor, MA) for 1 hour. All plates were then washed five times (1:20; 20X Wash Buffer Cat. No. X007; Arbor Assays), blotted dry, and $100 \mu 1$ of TMB (3, 3', 5, 5'-tetramethylbenzidine) (Moss Inc., Pasadena, MD) chromagen solution was added to each well. Plates were incubated for 30-45 min at room temperature without shaking, and the reaction stopped by adding 50 $\mu \mathrm{L}$ of a $1 \mathrm{~N} \mathrm{HCl}$ solution. Optical density was read in a plate reader at $450 \mathrm{~nm}$ (OPsys MR; Dynex Technologies, Chantilly, VA). The inter assay coefficient of variation (CV $\%$ ) for the high control was 3.0\%, and the low control CV\% was $2.9 \%$ ( $\mathrm{n}=31$ plates); intra-assay CV was maintained $<10 \%$ as all samples with duplicate CVs over $10 \%$ were reanalyzed. Assay sensitivity (based on $90 \%$ binding) was $0.14 \mathrm{ng} / \mathrm{ml}$.

All assays were previously validated for elephants by demonstrating: (1) parallelism between dilutions of pooled serum samples to the respective standard curve preparation and (2) significant (> 90\%) recovery of exogenous standard hormone added to pooled 
samples before analysis. The inter- and intra-assay coefficients of variation (CVs) were maintained below $15 \%$ and $10 \%$, respectively, for all assays and sample types.

Progestagen data were charted and visually inspected to determine ovarian cyclicity status based on progestagen patterns (Brown and Lehnhardt, 1997; Brown et al., 1999; Glaeser et al., 2012). Distributions of raw FGM data were non-normal based on the Shapiro-Wilk normality test (R package "stats"), and log-transformed data failed normality tests based on values of kurtosis and skewness (calculated in Excel). Therefore, data were analyzed with non-parametric tests performed on non-transformed raw data. Fecal sample sizes were unequal across habitat phases due to different durations of each phase, and unfortunately, all samples collected between February and October 2013 had to be discarded due to storage issues. Comparisons in median adrenal hormone concentrations between the three phases of the project (previous habitat, construction phase, new habitat) were made using a Kruskal-Wallis rank sum test (R package "stats"), with post-hoc pairwise comparisons using Dunn's Multiple Comparisons (R package "FSA") with Benjamini-Hochberg corrections applied for multiple comparisons (Benjamini and Hochberg, 1995). Comparisons of variability in adrenal hormone concentration between the phases were made using a Levene's test for equality of variance (R package "lawstat") modified to calculate deviations from the median (Brown and Forsythe, 1974) and with structural zeros removed (Hines and Hines, 2000). Although statistical power may be lower when outliers are present (Parra-Frutos, 2013), outliers were not removed for analysis because these data are biologically relevant. 


\section{$\underline{\text { Behavior and resource use }}$}

Behaviors were coded using video recordings rather than live observations to facilitate more detailed behavior assessment. Behavior data collection was designed to capture diurnal activity for both morning and afternoon periods on a weekly basis. Video was recorded every other week, on alternating Saturdays and Mondays, during two 2-hour time periods (10:00 - 12:00 and 14:00 - 16:00). Focal animals were recorded only from visitor viewing areas and were recorded in the order seen, left to right, to eliminate recording bias. Each focal animal was recorded for 2 minutes once every 30 minutes, beginning at the start of the hour and half hour, for a total of eight 2-minute recordings per day. Video was recorded only for self-initiated behavior to exclude keeper directed or modified behaviors. Actual breeding was not recorded because the opportunity to breed was limited to a subset of elephants. Video was recorded by a team of 14 Oregon Zoo volunteers trained on a protocol that ensured consistency between each phase of the project, and the PI reviewed raw video and conducted periodic training updates and inperson checks to ensure adherence to protocol.

A multi-level ethogram was designed to be widely applicable, with behavior categories at a high/overview level for long-term monitoring, detailed social behaviors organized by social function to assess changing social dynamics, and habitat-related behavior modifiers to assess resource use. (abbreviated version: Table 2; full version: Appendix C). The ethogram was constructed using published sources (Eisenberg et al., 1990; Kahl and Santiapillai, 2004; Olson, 2004; Greco et al., 2016) and unpublished studies at the Oregon Zoo, and included both state and event behaviors. Behaviors were described 
objectively (i.e., without interpretation of intent or purpose) such that they could be recognized by multiple observers without expertise in elephant behavior. Behavior categories were mutually exclusive and hierarchically organized by overarching categories such as social interactions, resource related behaviors, and solitary behaviors (Table 2). See Appendix $\mathrm{C}$ for the full ethogram that shows this nested structure.

Social interactions were the highest priority because social factors play an important role in the behavior and ecology of wild elephants, and proper social management can be used to promote positive welfare. Rare and unexpected behaviors were also included in the event of a major change in social structure (e.g., transfer in of an elephant). Although social interactions are mediated by various forms of communication (Vidya and Sukumar, 2005), with video observation methods we were not able to include acoustic communication in the behavioral repertoire, and olfactory communication was limited to trunk-related behaviors that were easier to discern and also have function in assessing reproductive state (Rasmussen, 1998; Slade et al., 2003). Resource related behaviors encompassed interactions with the physical environment and acquiring nutrition, which were important in evaluating efficacy of a new habitat. Aspects of the social and physical environments may be enriching, but were not classified as enrichment in this study; rather, enrichment was defined as non-food objects added to the environment by care staff which adhered to goals of providing stimuli and eliciting species-appropriate behaviors for the benefit of the animals (Shepherdson, 1994; Shepherdson et al., 1998). 
Table 2: Elephant Behavior Ethogram-abbreviated version

\begin{tabular}{|c|c|}
\hline Proximity & Definition \\
\hline Proximate & $\begin{array}{l}\text { Focal animal is within } 2 \text { body lengths of or in contact with another } \\
\text { individual. } \\
2 \text { body lengths defined as: } 2 \text { adult female body lengths (c.a. } 10 \mathrm{~m} \text { ) }\end{array}$ \\
\hline Proximity Not Visible & $\begin{array}{l}\text { Focal animal or other elephants are not visible enough to determine } \\
\text { proximity. }\end{array}$ \\
\hline Behavior Category & Definition \\
\hline Behavior Not Visible & Elephant or activity is not visible enough to determine the behavior. \\
\hline Social Interaction & $\begin{array}{l}\text { Interacting with another elephant in a social context, either with } \\
\text { physical contact (e.g., trunk twine, play) or without physical contact } \\
\text { but within } 2 \text { body lengths (e.g., sharing food, displace). }\end{array}$ \\
\hline $\begin{array}{l}\text { Food Object } \\
\text { Interaction }\end{array}$ & $\begin{array}{l}\text { Interacting with any item that distributes food, either permanent or } \\
\text { keeper-provided. }\end{array}$ \\
\hline Feeding/Drinking & $\begin{array}{l}\text { Gathering and putting any food item in the mouth without interacting } \\
\text { with an object. Drinking water, not bathing. }\end{array}$ \\
\hline $\begin{array}{l}\text { Enrichment (non-food) } \\
\text { Object Interaction }\end{array}$ & $\begin{array}{l}\text { Interacting with non-food items that are not permanent (e.g., } \\
\text { firehose ball or braid). }\end{array}$ \\
\hline $\begin{array}{l}\text { Habitat Feature } \\
\text { Interaction }\end{array}$ & $\begin{array}{l}\text { Interacting with features that are permanent in the habitat (e.g., } \\
\text { dusting, bathing, digging, rubbing body, climbing on logs, } \\
\text { investigating features with feet or mouth). }\end{array}$ \\
\hline $\begin{array}{l}\text { Repetitive Behaviors } \\
\text { (Stereotypy) }\end{array}$ & $\begin{array}{l}\text { Motor: Repeatedly performing a behavior for } 3 \text { or more consecutive } \\
\text { repetitions without interruption (e.g., route tracing). } \\
\text { Non motor stereotypy: Performing the behavior for } 5 \text { seconds or } \\
\text { more. }\end{array}$ \\
\hline Locomotion & $\begin{array}{l}\text { Walking or running (fast walking) more than } 2 \text { body lengths in any } \\
\text { direction, without stopping for } 3 \mathrm{sec} \text { or more. }\end{array}$ \\
\hline Stationary & $\begin{array}{l}\text { Any stationary state without engaging in another behavior for } 3 \mathrm{sec} \\
\text { or longer. Standing, walking or shuffling (without moving } 2 \text { body } \\
\text { lengths), sitting, kneeling, lying (prone or sternal). }\end{array}$ \\
\hline
\end{tabular}


Behaviors were coded from video using Observer XT 11.5 (Noldus Information Technology) by the PI and five observers trained to meet $85 \%$ inter-observer reliability criteria. Behaviors were recorded using instantaneous focal animal sampling (Martin and Bateson, 1993), with a sampling interval of 30 seconds. The sampling interval was determined using a method modified from Margulis and Westhus (2008), whereby behaviors were initially scored at $30 \mathrm{sec}$ then subsampled to lengthen the interscan interval, and then the rates of behaviors were compared across different intervals. At every sampling interval, observers coded keeper presence to ensure only self-initiated behaviors were included in the analysis, proximity of focal to other elephants, and a single behavior within the top-most behavior category that applied, for a total of 4 behaviors per 2-minute observation. Behavior of the focal animal was visible in $86 \%$ of the samples (i.e., not visible 14\%). Social behaviors that required interpretation in order to categorize correctly as affiliative, agonistic, or reproductive (e.g., playing v. sparing, rump present as submissive v. reproductive context) were reviewed by the PI. Coding was conducted over a period of approximately two years, so interim tests were conducted to ensure reliability criteria was maintained. Interim tests using 20 observations with 5 comparisons each resulted in an average of $89 \%$ inter-reliability for all observers (range $85 \%$ to $92 \%$ ), which equated to a pooled Cohen's kappa of 0.82 and a confidence interval of 0.72 to 0.91 . Intra-observer reliability and drift were not directly tested; however, the PI conducted random checks every two weeks on approximately $10 \%$ of the observations and reviewed at least $25 \%$ of the observations. 
Observation data were exported to Excel for charting and analysis in R. Since behaviors were defined as mutually exclusive and thus were not independent of each other, a G-test of independence (Fowler et al., 1999; Plowman, 2008) in the R package "DecTools" (Signorell et al., 2019) was used to investigate changes in activity budgets between the new and previous habitats for each elephant and all elephants combined. Within behavior categories, the frequencies of recorded behavior were not normally distributed based on the Shapiro-Wilk test; therefore, a non-parametric Mann-Whitney U-test was used for all pairwise comparisons of centrality between the new and previous habitats. Comparisons were made only for those behavioral categories that occurred at frequencies greater than $2 \%$ in at least one habitat. Significance was assessed at the 0.05 level. The number of behaviors expressed ('behavior richness') was compared using specific behaviors rather than categories.

Behavioral diversity was measured using the Shannon-Wiener (or Shannon-Weaver) diversity Index and a Hill Number analysis in the R package "vegan". Specific behaviors rather than categories were included in the analyses. The value of Shannon-Wiener Diversity H-index depends on both the number of behaviors ("richness") and on the equality of the distribution of time spent in each behavior. The higher the H-index the greater the number of behaviors and the more homogeneous their distribution in the activity budget (Shepherdson et al., 1993; Fowler et al., 1999). However, for this species a homogenous distribution of the behavioral repertoire would not be expected, and a higher $\mathrm{H}$-index would not necessarily indicate improved welfare. By assuming a greater diversity (higher H-index) equates to better welfare, we are by extension assuming that 
all behaviors/categories have equal ability to contribute to positive welfare (Cronin and Ross, 2019). For example, within a group of elephants we would expect expression of both affiliative and agonistic or dominance/submissive behaviors but at different proportions, and an individual or group experiencing equal proportions may have a more negative affective state. The Hill Number analysis generalizes a family of diversity indices (Shannon-Wiener, Simpson's, and total number of species) to achieve an order of diversity between two or more communities (Tóthmérész, 1995). By generalizing these indices, the analysis can be more weighted towards relative species richness or abundance. The Hill number values, or Renyi diversities, represent the number of equally abundant species necessary to produce the observed value of diversity. According to this analysis, the diversity of one community is higher than another only if all Renyi

diversities are higher. In comparing behavior diversity in the previous and new habitats, if some Renyi diversities are higher and some lower, this means that no two indices Shannon, Simpson, or total number - agreed on the order of behavioral diversity.

\section{Results}

\section{Walking distance}

Female1 (Fig. 3) doubled her mean daily walking distance from $7.6 \mathrm{~km}(4.7 \mathrm{mi})$ in the previous habitat to $15.4 \mathrm{~km}(9.6 \mathrm{mi})$ in the new habitat $(\mathrm{W}=18, \mathrm{p}=0.024)$. Female2 walked similar distances in both habitats, with a mean daily walking distance of $17.3 \mathrm{~km}$ $(10.7 \mathrm{mi})$ in the previous habitat and $17.6 \mathrm{~km}(10.9 \mathrm{mi})$ in the new habitat $(\mathrm{W}=11, \mathrm{p}=$ 1.0) (Fig. 3). Across all seasons in the new habitat, Female1 averaged $13.8 \mathrm{~km}(8.6 \mathrm{mi})$ 
per day, with a maximum of $20.8 \mathrm{~km}(12.9 \mathrm{mi})$; and Female2 averaged $16.2 \mathrm{~km}(10.1 \mathrm{mi})$ per day, with a maximum of $23.7 \mathrm{~km}$ (14.7 mi). M-juvenile had a mean daily walking distance of about $10 \mathrm{~km}$ (6 mi) per day (Fig. 3), but this result underestimates his actual walking distance. We were able to collect data on M-juvenile only from January to May in 2016, and on the days when he wore the GPS bracelet, he chose to spend most of his time indoors resulting in a maximum of 9 hours of data recording. We know that the elephants actually walked farther than GPS calculations indicated since we counted movement only in outside habitats due to GPS receiver limitations, and they also walked and exercised regularly in the large indoor habitat.

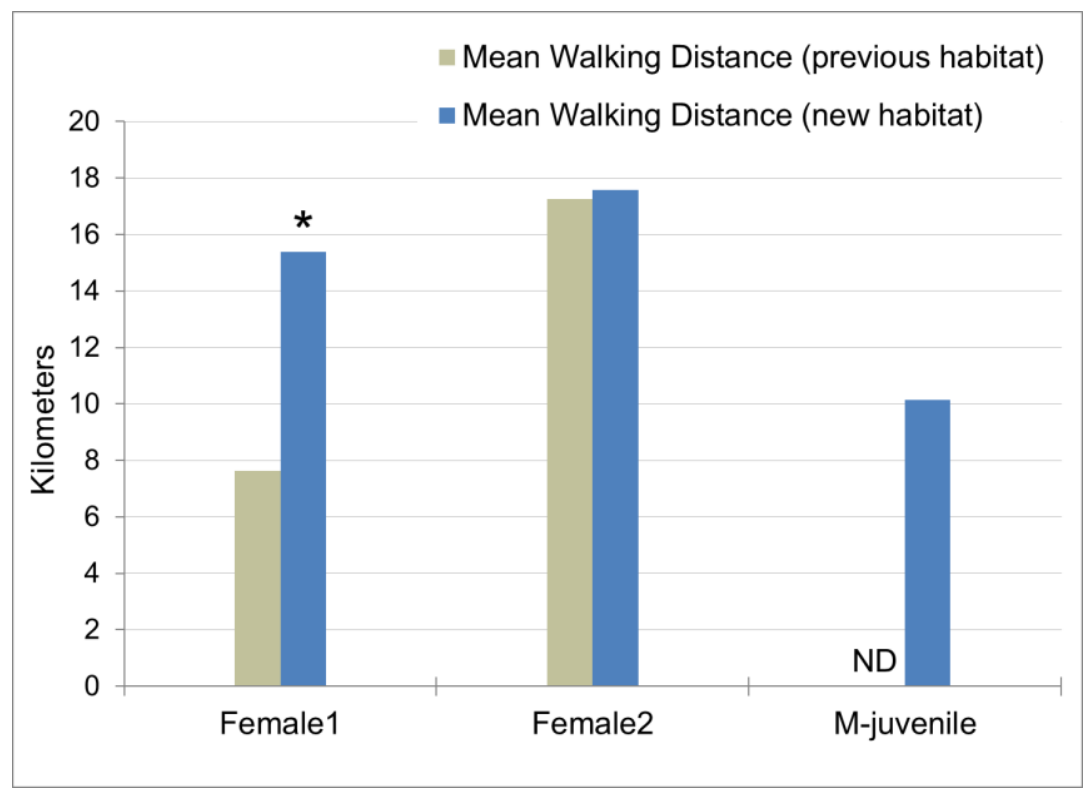

Fig 3: Mean daily walking distance comparing the previous habitat and new habitat.

Asterisk (*) above bars indicates statistically significant differences between habitats. ND indicates no data. 


\section{$\underline{\text { Space use }}$}

GPS mapping (Fig. 4) shows GPS data points where elephants walked in the outdoor portion of the new habitat. Visual inspection shows they are using all habitat areas rather than concentrating their use in a few preferred areas, with similar usage across seasons. The space-use patterns clearly suggest this is the result of the increased variety of feeding and enrichment opportunities designed into the habitat. Movement in the new habitat was more self-directed than in the previous habitat. In the new habitat, elephants had outdoor access for more than 20 hours a day in every month of the year except January, and individuals chose to spend anywhere from 6 to 20 hours outdoors. Even on colder days, some individuals chose to leave the heated indoor area and spend more time outdoors, while other members of the herd chose to remain indoors. 


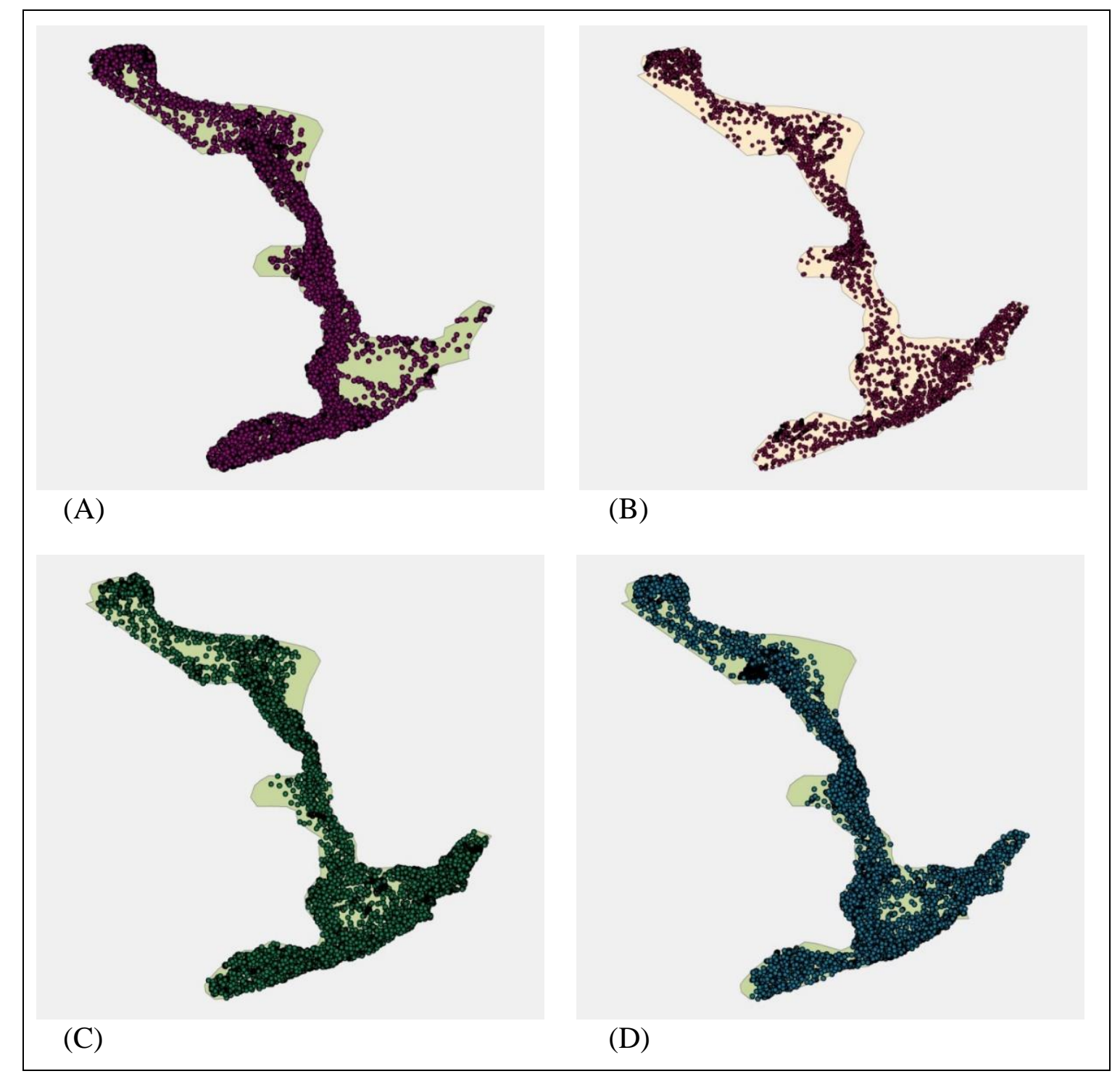

Fig 4: Representative examples of GPS data in outdoor areas of the new habitat at different times of year.

(A) Female2 using areas nearest public viewing areas, dirt mounds, and feeders at the top of the hill in the north habitat (August 2016). (B) M-juvenile using the entire habitat including pools and dirt mounds over a 12-hour period (May 2016). (C) Female1 using the entire habitat (August 2016). (D) Female1 using the entire habitat in cooler weather (November 2016).

\section{$\underline{\text { Reproductive and adrenal hormones }}$}

All females continued regular ovarian cycles throughout the construction phase and in the new habitat. M-juvenile exhibited signs of his first musth with temporal gland secretions coinciding with elevated testosterone at age six, during the construction phase. 
All elephants showed significant differences in FGM median concentrations and variability across habitat phases, with the highest levels and greatest interquartile range (IQR) during the construction phase, and lower levels and IQR in the previous and/or the new habitats (Fig. 5, Table 3). Statistics are provided in Tables 4 and 5. 


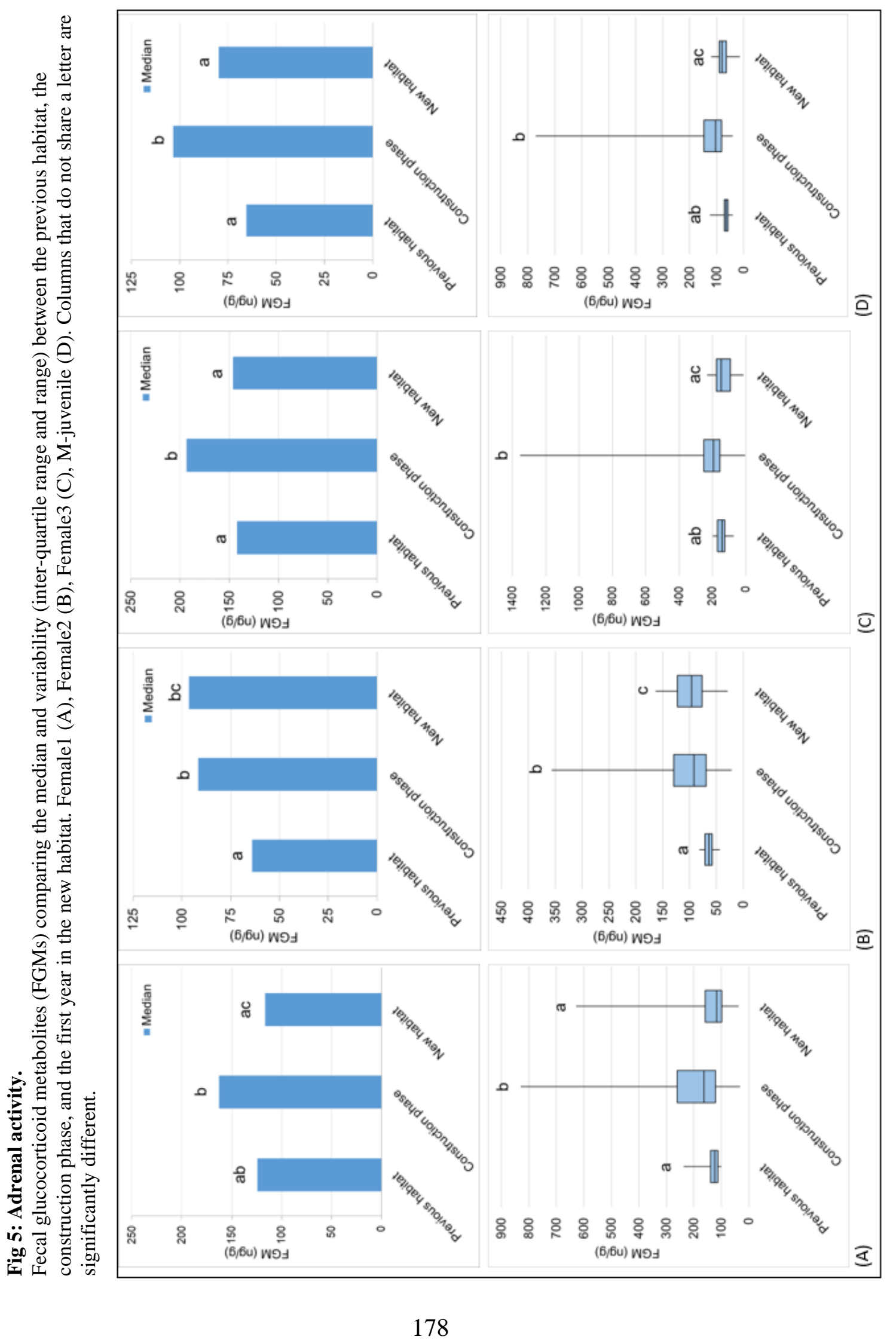




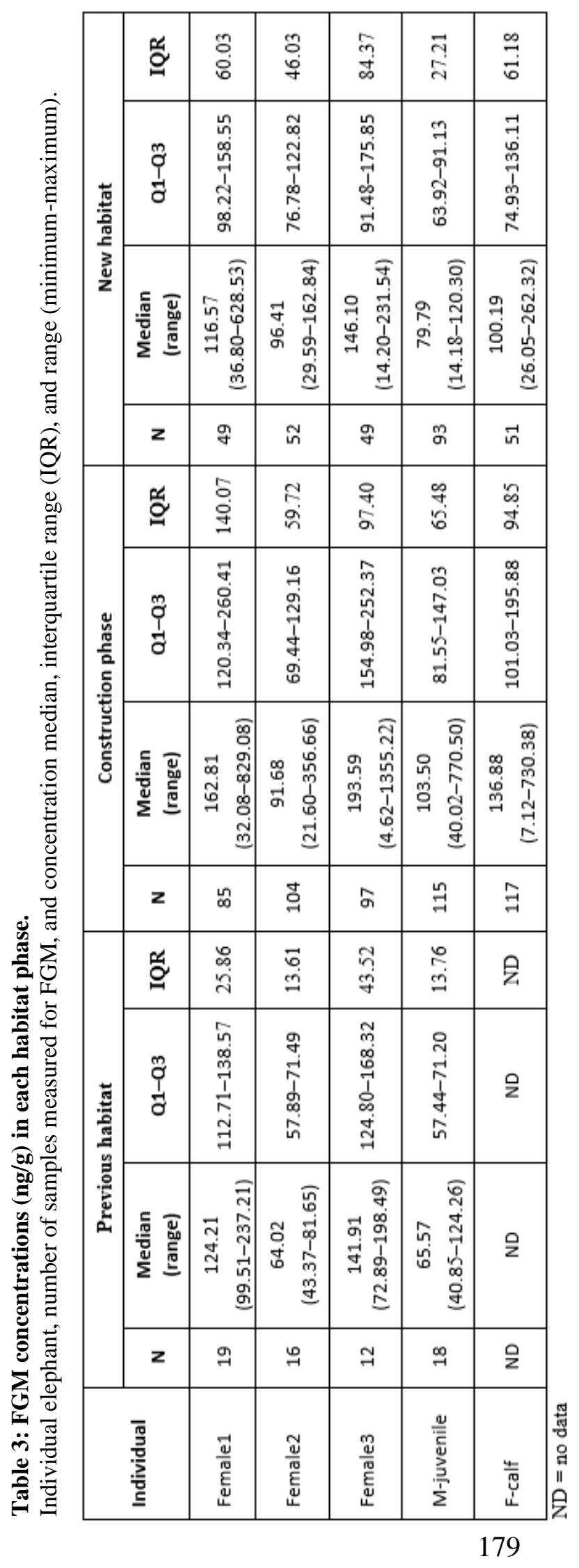


Female1 (Fig. 5A) showed higher median FGM concentration in the construction phase compared to the new habitat, a higher but not significantly different concentration in the construction phase compared to the previous habitat, and similar concentrations between the new and previous habitats (Table 4). Variability was highest in the construction phase compared to both the previous and new habitats, and higher but not significantly different in the new habitat compared to the previous habitat (Table 5).

Female3 (Fig. 5C) and M-juvenile (Fig. 5D) showed higher median FGM concentration in the construction phase compared to both the previous and new habitats, and similar concentrations between the new and previous habitats (Table 4). Variability was highest in the construction phase, but differed significantly only compared to the new habitat, with no significant difference between the new and previous habitats (Table 5). Female3 showed the highest median concentrations compared to other individuals, as well as the highest IQR, except during the construction phase when Female1 showed the highest IQR (Table 3).

Female2 (Fig. 5B) exhibited a somewhat different pattern, showing continued increased levels of adrenal activity in the new habitat. However, based on visual inspection of her hormone data, she did show a decline in FGM concentrations immediately following introductions to new habitat areas. Variability was highest in the construction phase, followed by the new habitat, with lowest variability in the previous habitat (Table 5). 
F-calf was born only months prior to the start of construction and therefore FGMs were compared only between the construction phase and the new habitat. As with Female1, Female3, and M-juvenile, she showed higher median and greater variability in adrenal activity during the construction phase (Tables 4 and 5). This study spanned ages 1 to 4 years for F-calf (Fig. 6), and it is not known how adrenal activity changes during early development in elephants; however, her concentrations showed intermediate levels compared to the other elephants (Table 3).

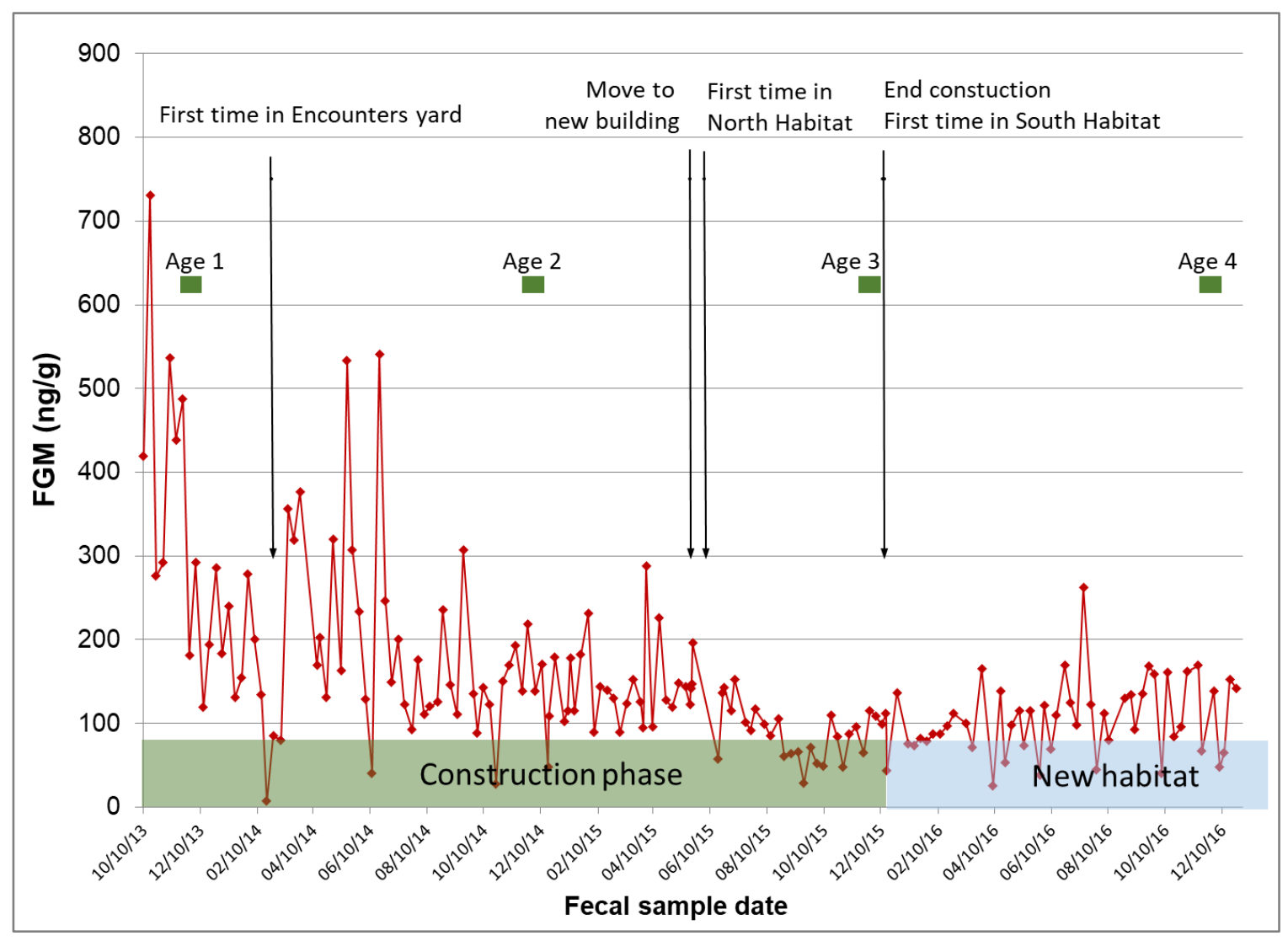

Fig 6: Hormone profile of FGM concentrations for F-calf showing temporal relationship of age and habitat phases. 
Table 4: Statistical results comparing median FGM concentrations across and between habitat phases.

Degrees of freedom (df) was 1 in all pair-wise comparisons.

\begin{tabular}{|c|c|c|c|c|c|}
\hline \multirow[b]{2}{*}{ Individual } & \multirow[b]{2}{*}{ Kruskal-Wallis test } & \multicolumn{4}{|c|}{ Dunn's Post-hoc Pairwise Comparisons } \\
\hline & & $\begin{array}{l}\text { Previous } \\
\text { habitat }\end{array}$ & $\begin{array}{c}\text { Construction } \\
\text { phase }\end{array}$ & $\begin{array}{c}\text { New } \\
\text { habitat }\end{array}$ & $\begin{array}{c}\text { Dunn's Multiple } \\
\text { Comparisons }\end{array}$ \\
\hline \multirow[t]{3}{*}{ Female2 } & \multirow{3}{*}{$\begin{array}{c}\chi^{2}=16.10, \mathrm{df}=2, \\
\mathbf{p}<0.001\end{array}$} & $\mathrm{X}$ & X-higher & & $Z=3.93, p<0.001$ \\
\hline & & & $X$ & $x$ & $Z=0.06, p=0.949$ \\
\hline & & $\mathrm{X}$ & & X-higher & $Z=3.66, p<0.001$ \\
\hline \multirow[t]{3}{*}{ Female1 } & \multirow{3}{*}{$\begin{array}{c}\chi^{2}=16.04, \mathrm{df}=2, \\
\mathbf{p}<0.001\end{array}$} & $x$ & $x$ & & $Z=1.90, p=0.085$ \\
\hline & & & X-higher & $\mathrm{X}$ & $Z=3.90, p<0.001$ \\
\hline & & $\mathrm{X}$ & & $\mathrm{X}$ & $Z=-0.80, p=0.425$ \\
\hline \multirow[t]{3}{*}{ Female3 } & \multirow{3}{*}{$\begin{array}{c}\chi^{2}=30.61, d f=2, \\
\mathbf{p}<0.001\end{array}$} & $\mathrm{x}$ & X-higher & & $Z=2.81, p=0.007$ \\
\hline & & & X-higher & $x$ & $Z=5.22, \mathbf{p}<\mathbf{0 . 0 0 1}$ \\
\hline & & $x$ & & $x$ & $Z=-0.17, p=0.87$ \\
\hline \multirow[t]{3}{*}{ M-juvenile } & \multirow{3}{*}{$\begin{array}{c}\chi^{2}=51.64, \mathrm{df}=2, \\
\mathbf{p}<0.001\end{array}$} & $\mathrm{X}$ & X-higher & & $Z=5.61, p<0.001$ \\
\hline & & & X-higher & $x$ & $Z=5.55, \mathbf{p}<\mathbf{0 . 0 0 1}$ \\
\hline & & $\mathrm{x}$ & & $\mathrm{x}$ & $Z=1.83, p=0.067$ \\
\hline F-calf & $\begin{array}{c}\chi^{2}=51.81, \mathrm{df}=1, \\
\mathbf{p}<0.001\end{array}$ & ND & X-higher & $x$ & ND \\
\hline
\end{tabular}

$\mathrm{ND}=$ no data 
Table 5: Statistical results comparing variability in FGM concentrations across and between habitat phases.

Degrees of freedom (df) was 1 in all pair-wise comparisons.

\begin{tabular}{|c|c|c|c|c|c|}
\hline \multirow[b]{2}{*}{ Individual } & \multicolumn{5}{|c|}{ Levene's Test for Equality of Variance } \\
\hline & Across all phases & $\begin{array}{l}\text { Previous } \\
\text { habitat }\end{array}$ & $\begin{array}{c}\text { Construction } \\
\text { phase }\end{array}$ & $\begin{array}{c}\text { New } \\
\text { habitat }\end{array}$ & $\begin{array}{c}\text { Pairwise } \\
\text { Comparisons }\end{array}$ \\
\hline \multirow[t]{3}{*}{ Female2 } & \multirow{3}{*}{$\begin{array}{c}F=6.82, d f=2, \\
p=0.001\end{array}$} & $\mathrm{x}$ & X-higher & & $F=7.61, p=0.007$ \\
\hline & & & X-higher & $\mathrm{X}$ & $F=5.75, p=0.018$ \\
\hline & & $\mathrm{x}$ & & X-higher & $F=11.61, p=0.001$ \\
\hline \multirow[t]{3}{*}{ Female1 } & \multirow{3}{*}{$\begin{array}{c}F=6.19, \mathrm{df}=2, \\
\mathbf{p}=\mathbf{0 . 0 0 3}\end{array}$} & $x$ & X-higher & & $F=5.33, p=0.023$ \\
\hline & & & X-higher & $\mathrm{X}$ & $F=7.82, p=0.006$ \\
\hline & & $\mathrm{x}$ & & $\mathrm{X}$ & $F=0.72, p=0.400$ \\
\hline \multirow[t]{3}{*}{ Female3 } & \multirow{3}{*}{$\begin{array}{c}F=2.89, d f=2, \\
p=0.059\end{array}$} & $x$ & $x$ & & $F=1.71, p=0.194$ \\
\hline & & & X-higher & $x$ & $F=4.06, p=0.046$ \\
\hline & & $x$ & & $x$ & $F=2.83, p=0.136$ \\
\hline \multirow[t]{3}{*}{ M-juvenile } & \multirow{3}{*}{$\begin{array}{c}F=5.26, d f=2, \\
p=0.006\end{array}$} & $x$ & $x$ & & $F=3.24, p=0.074$ \\
\hline & & & X-higher & $x$ & $F=7.36, p=0.007$ \\
\hline & & $\mathrm{X}$ & & $\mathrm{x}$ & $F=1.51, p=0.224$ \\
\hline F-calf & $\begin{array}{c}F=8.09, d f=1 \\
p=0.005\end{array}$ & ND & X-higher & $\mathrm{X}$ & \\
\hline
\end{tabular}

$\mathrm{ND}=$ no data

\section{Behavior}

\section{Activity budgets}

With all elephants combined, elephants spent the majority of their time in the new habitat seeking food and feeding (40\%), socializing and interacting with their environment in a social context (23\%), and locomoting (18\%), with a smaller portion of their time spent interacting with enrichment objects and features in their environment in a non-social context, e.g., in the pool alone (5.7\%) (Fig. 7A). 
Activity budgets differed significantly between the new ( $n=4107$ visible samples $)$ and previous $(\mathrm{n}=5609$ visible samples $)$ habitats for all elephants $(\mathrm{N}=5)$ combined $(\mathrm{G}$-test of independence: $\mathrm{G}=1265.80, \mathrm{df}=7, \mathrm{p}<0.001$ ) and for each individual (Female2: $\mathrm{G}=$ 501.03, df = 7, $\mathrm{p}<0.001 ;$ Female1: $\mathrm{G}=331.11, \mathrm{df}=7, \mathrm{p}<0.001 ;$ Female3: $\mathrm{G}=133.08$, df $=7, p<0.001 ;$ F-calf: $\mathrm{G}=151.29, \mathrm{df}=7, \mathrm{p}<0.001 ;$ M-juvenile: $\mathrm{G}=544.93, \mathrm{df}=7$, $\mathrm{p}<0.001)$. Appendix B shows all pairwise comparisons between the new and previous habitats.

The average percentage of time spent seeking food and eating during staffed hours was similar between the new habitat (40\%) and the previous habitat (37\%); however, interacting with items that disburse food (e.g., timed feeders, overhead hay nets) increased six-fold in the new habitat (from $3.8 \%$ to $24.5 \%$ ), while feeding without interaction with a food delivery item decreased by half (from 32.7\% to 15.3\%) (Fig. 7A). This shows that a switch occurred from primarily eating food provided directly by keepers in the previous habitat to utilizing the food-delivery features that require manipulation and extend food disbursement across time and space.

Locomotion increased from an average of $13.4 \%$ to $18.2 \%$ in the new habitat, while stationary behaviors decreased (3.7\% decrease). Repetitive behaviors were exhibited by two adult females, both in a locomotor form of pacing or route tracing, with a decrease in average time spent from $7.9 \%$ in the previous habitat to $4.0 \%$ in the new habitat. Time spent performing all active behaviors (social behaviors, interaction with food-delivery 
and non-food enrichment objects and habitat features, feeding/drinking, and locomotion) increased for all elephants combined (4\% increase) and for all females (Fig. 8).
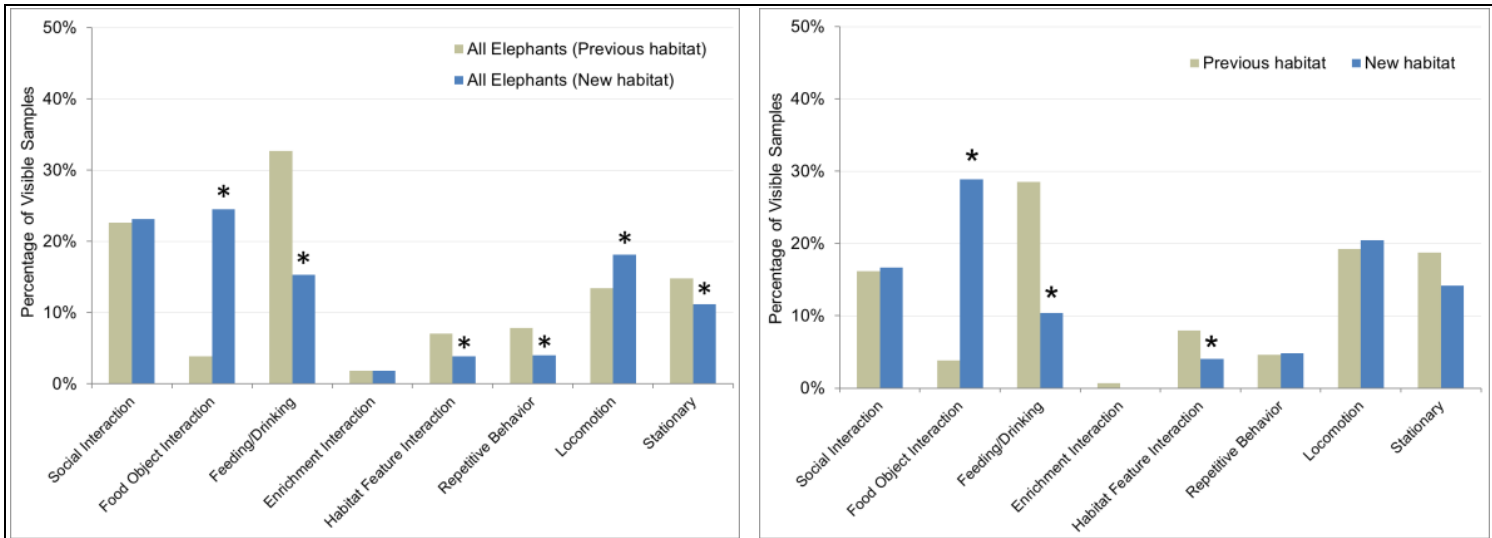

(A)

(B)
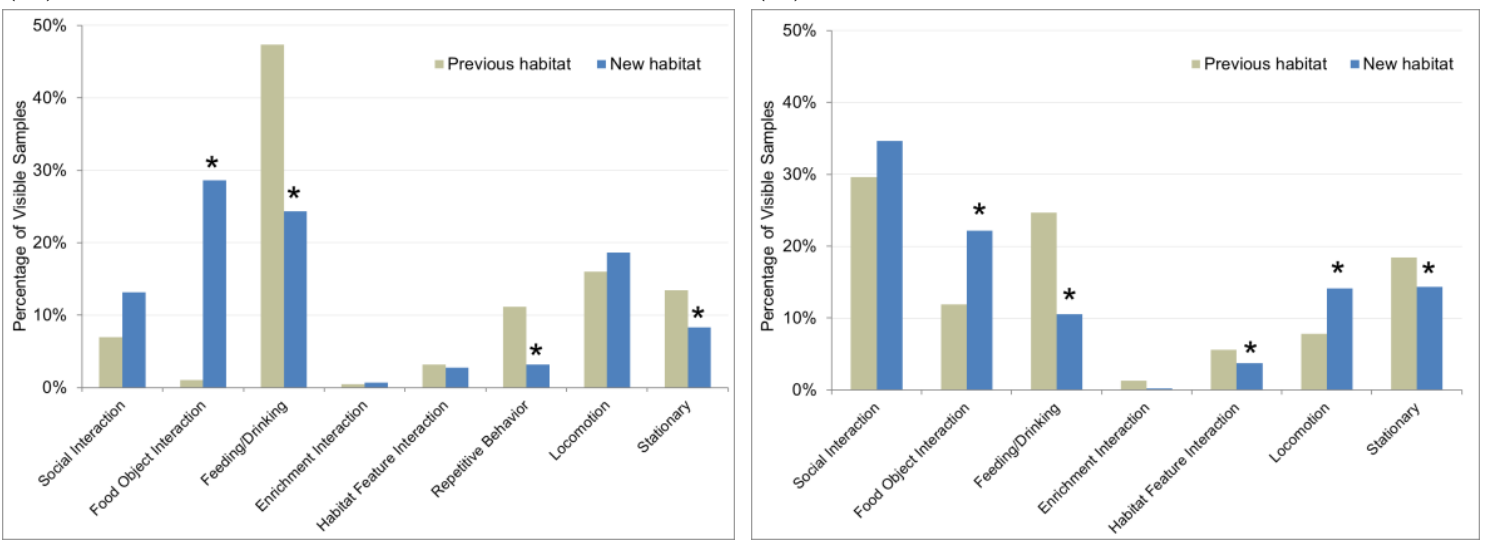

(C)

(D)
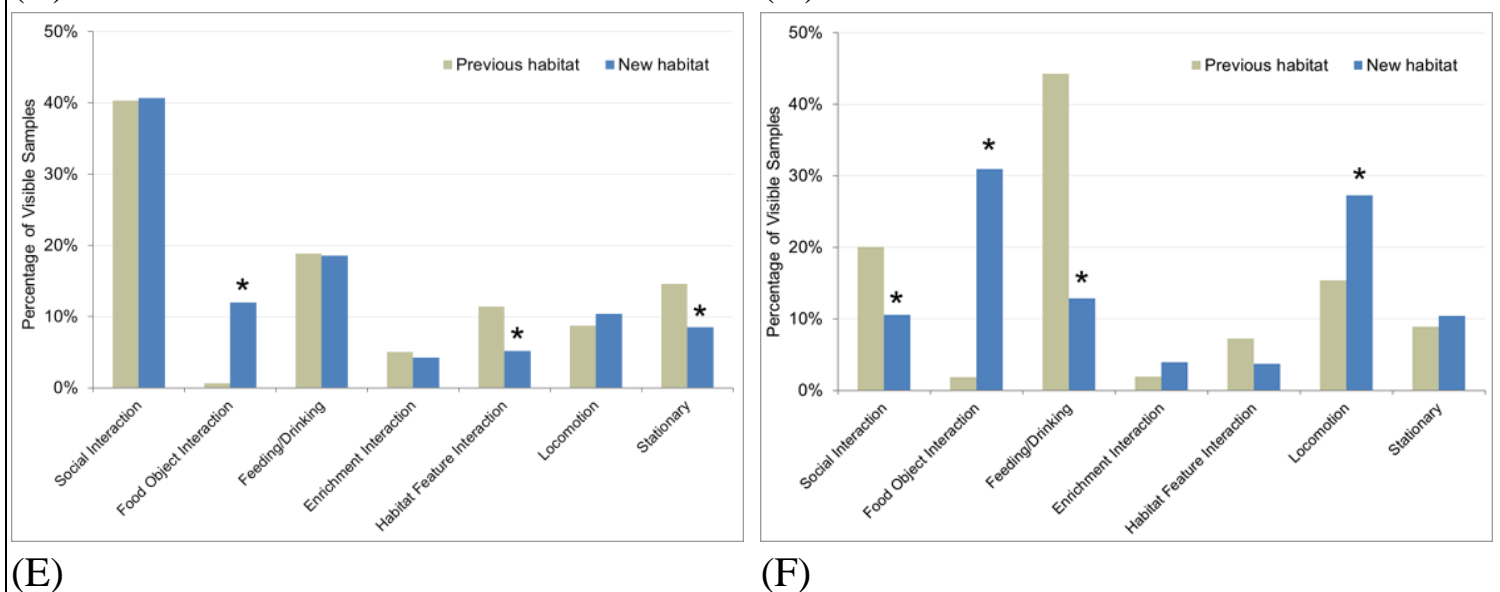

(E)

(F)

Fig 7: Activity budgets.

Percentage of observation samples engaged in each behavioral category, comparing the previous habitat and new habitat for all elephants combined and for individual elephants. All elephants (A), Female1 (B), Female2 (C), Female3 (D), F-calf (E), M-juvenile (F). Asterisk (*) above bars indicates statistically significant differences between habitats when comparing the same behavioral category. 


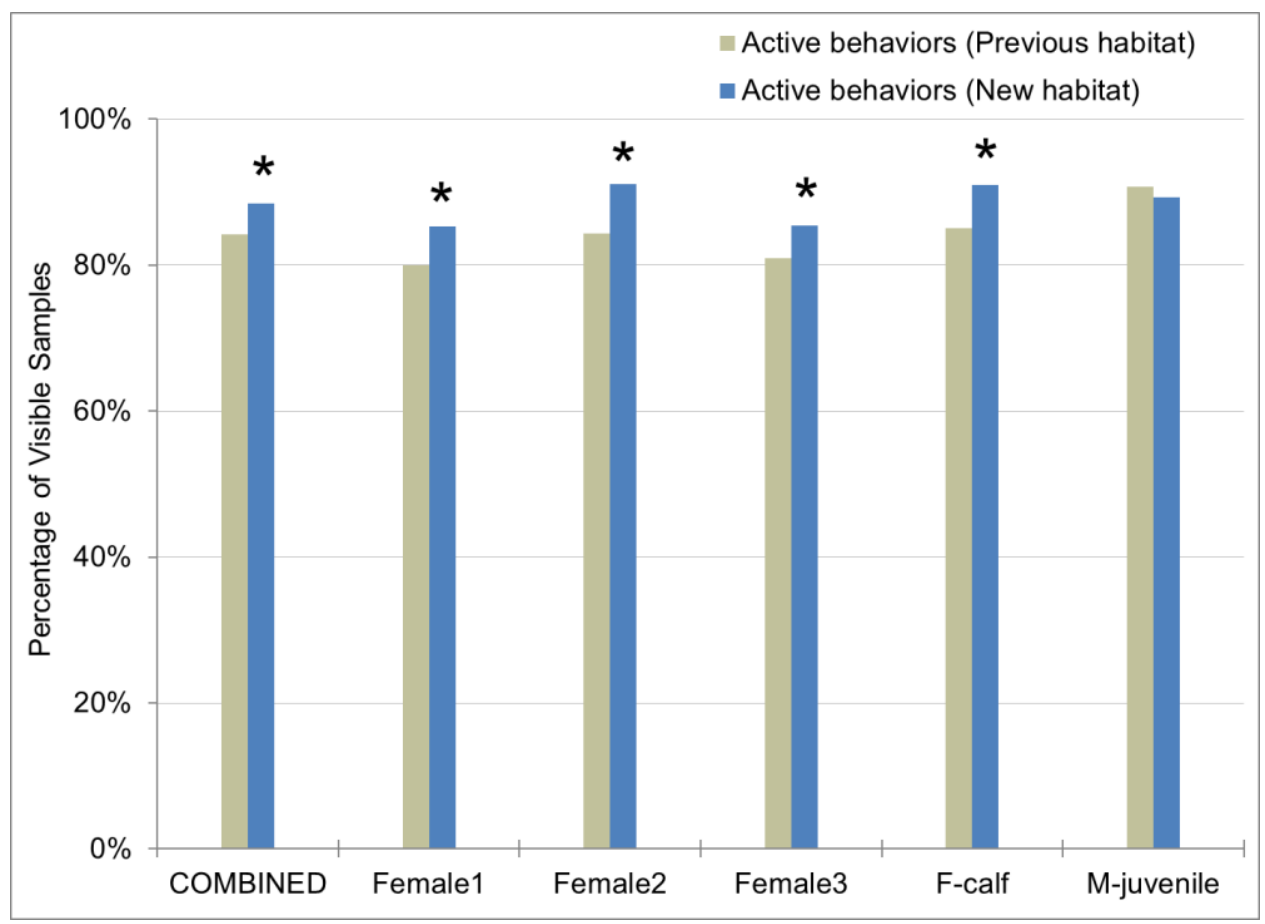

Fig 8: Active behaviors.

Percentage of observation samples exhibiting active (non-repetitive) behaviors, comparing the previous habitat and new habitat for individual elephants and all elephants combined. Asterisk (*) above bars indicates statistically significant differences between habitats when comparing proximity to herdmates using the Mann-Whitney U-test.

\section{Behavior changes for individual elephants}

Female1 (Fig. 7B) switched to feeding primarily from food-delivery resources in the new habitat (26\% increase), but learning this strategy appeared to be more challenging for her and the switch to more independent feeding took longer than it did for the other elephants. During the first several weeks, Female1 spent a substantial amount of time near gates, apparently in anticipation of keepers delivering food rather than seeking food on her own. Keepers attempted to "teach" her to eat out of the feeders by calling her to the feeders, which may have unintentionally prolonged the transition. She showed few repetitive behaviors (less than 5\% of her time) in the previous and new habitat, and such behaviors occurred in infrequent bouts. We saw a marked decrease in bouts of repetitive 
behavior as she began seeking food on her own. Time spent performing active behaviors (Fig. 8) increased (5\% increase). In contrast to the GPS monitoring results for Female1, behavior results indicated that the amount of time spent in locomotion was not significantly different between the two habitats.

Female2 (Fig. 7C) switched from spending almost no time interacting with food-delivery resources in the previous habitat to foraging from various food-delivery resources throughout the new habitat ( $29 \%$ of her time). However, she still spent a larger portion of her time than other elephants ( $24 \%$ of her time) feeding from food that was available without object manipulation, such as live vegetation, browse and hay left by other elephants near food-delivery resources. She showed a clear, but not significant, increase in social interaction (6.2\% increase, almost double). Time spent performing active behaviors (Fig. 8 ) increased significantly (7\% increase), and repetitive behaviors decreased significantly in the new habitat from about $11 \%$ of her time in the previous habitat to $3 \%$ of her time.

Female3 (Fig. 7D) switched readily to feeding primarily from food-delivery resources in the new habitat (10\% increase); however, in the previous habitat she already spent more time manipulating food-delivery resources than other elephants, so the increase for her was not as large as the other elephants. Locomotion increased in the new habitat (6\% increase, almost doubled) and time spent stationary decreased (4\% decrease). Female3 showed less than $0.05 \%$ of her time in repetitive behaviors in the previous habitat, and showed no repetitive behaviors in the new habitat. 
F-calf (Fig. 7E) adapted most readily to the new habitat, and was usually the first elephant to venture into new habitat areas. F-calf quickly learned to seek food from a diversity of feeding methods in the new habitat, although she still spent a larger portion of her time feeding from food that was available without object manipulation. In both habitats she spent a larger portion of her time interacting with habitat features than other elephants, but the percentage of time decreased from $11 \%$ in the previous habitat to $5 \%$ in the new habitat. Her time spent performing active behaviors increased (6\% increase), while time spent stationary decreased ( $6 \%$ decrease). F-calf showed no repetitive behaviors in either habitat.

M-juvenile (Fig. 7F) also switched from spending almost no time interacting with fooddelivery resources in the previous habitat to seeking food from a diversity of feeding methods in the new habitat. Locomotion also increased in the new habitat (12\% increase). M-juvenile was the only elephant to exhibit a decrease in social interactions (by 9.5\%, almost half). M-juvenile showed no repetitive behaviors in either habitat.

\section{Behavioral diversity}

In comparing the presence/absence of behaviors exhibited, elephants were observed exhibiting 29 behaviors in the new habitat and 39 in the previous habitat, with 10 behaviors observed only in the previous habitat while none were unique to the new habitat. Behaviors unique to the previous habitat were social interactions related to protecting the calf and interactions with adult bulls related to courtship behavior and arousal (e.g., (claim calf, intervene, rump present of female to adult male, pre-mount, 
spinning and urinating). The Shannon-Wiener diversity index showed a higher diversity in the previous habitat, when comparing all behaviors in the ethogram (previous: $\mathrm{H}$ $=2.28$, new: $\mathrm{H}=2.16$ ) and also when including only social behaviors (previous=2.28, new $=1.54)$. Results of the Hill Number analysis indicate that commonly-used indices of Shannon-Wiener, Simpson's and total number of behaviors do not agree on the diversity order because the diversity profiles are intersecting (Fig. 9). In this case, the ShannonIndex and total number of behaviors agree that diversity was highest in the previous habitat, which may suggest that the higher diversity is a result of rare behaviors not weighted by the Simpson's index.

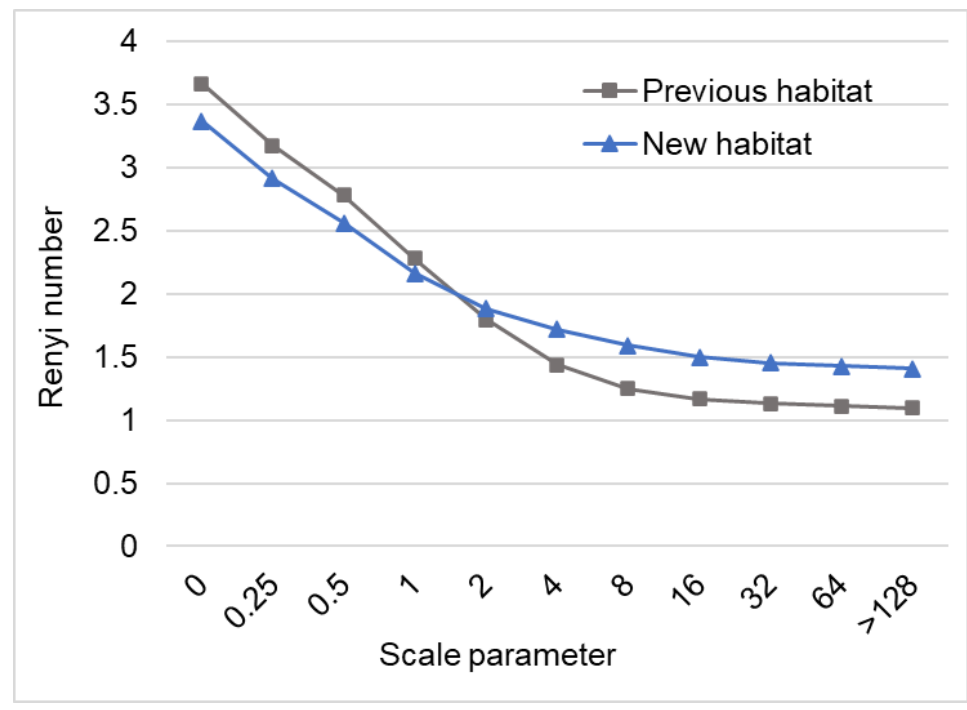

Fig 9: Hill Number analysis results showing intersecting diversity profiles

\section{Social behaviors and partners}

The percentage of time spent in proximity of other elephants decreased for all individuals (average $25 \%$ decrease) in the new habitat ( $\mathrm{n}=4499$ samples) compared to the previous habitat $(\mathrm{n}=5525$ samples) (Fig. 10). Female3 and her calf (F-calf) had the highest percentage of time spent proximate to another elephant in both the previous and new 
habitats, followed by Female1. In the previous habitat, Female2 spent the least percentage of time in close proximity to other elephants, but M-juvenile showed the largest decrease in proximity (by more than half) and was the least proximate in the new habitat.

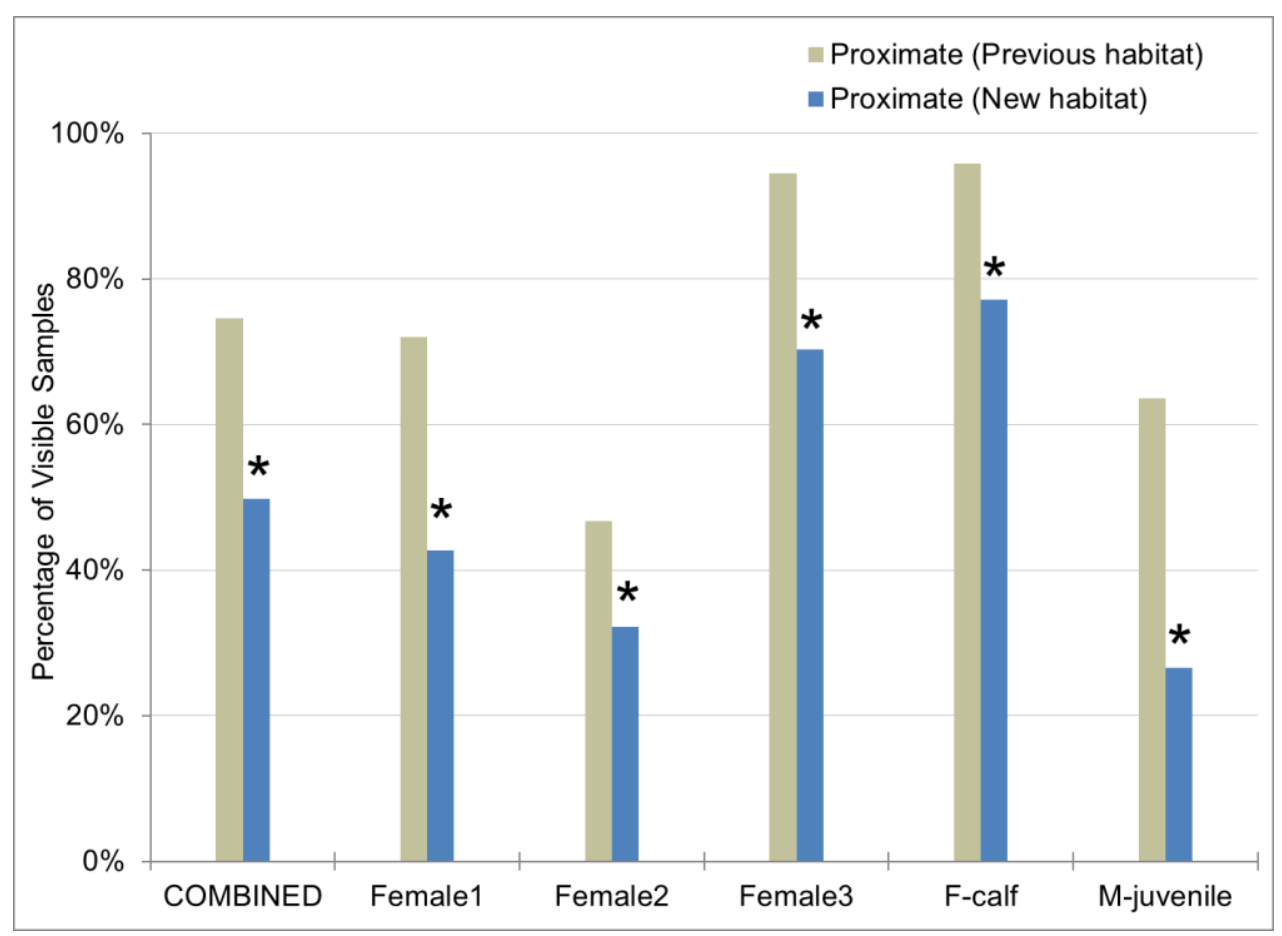

Fig 10: Proximity to herdmates.

Percentage of observation samples in proximity to herdmates, comparing the previous habitat and new habitat for individual elephants and all elephants combined. Asterisk $(*)$ above bars indicates statistically significant differences between habitats when comparing proximity to herdmates using the Mann-Whitney U-test.

Figure 11 shows details about the social relationships among this group of elephants. Although differences in proportion of time spent engaging in active social interaction were not statistically significant except for M-juvenile (Fig. 7), there were significant differences between habitats in the social partners in these interactions. The pattern of herdmates for social interaction reflected proximity, which was expected because limitations of video observation dictated that social interaction required proximity. 
However, being proximate did not imply active social interaction; and therefore, the number of samples proximate to a given herdmate was greater than or equal to the number of samples having social interaction with that herdmate, which explains why percentage of time is not consistent between proximity and social interaction in each habitat. Also, the adult females and M-juvenile spent between $2 \%$ and $9 \%$ proximate to and interacting with adult bulls in the previous habitat, and only one bull was reintegrated with the herd in the new habitat and only for 6 months.

Female1 (Fig. 11A) was proximate to and interacted with all of her herdmates more evenly in the previous habitat, with only a $10 \%$ difference across herdmates. In the new habitat, her time spent proximate to and interacting with Female2 and M-juvenile decreased, and she spent a larger portion of her time with Female3 and F-calf.

Interestingly, the herdmates with whom Female2 (Fig. 11B) spent the most time in proximity in the new habitat were opposite to those in the previous habitat. In the previous habitat she spent the largest portion of her time proximate to and interacting with Female1 and M-juvenile, whereas in the new habitat she spent the largest portion proximate to and interacting with Female 3 and F-calf.

Female3 (Fig. 11C) spent the majority of her time with her calf in both habitats. The small but significant increase in proximity to F-calf may indicate a difference in the median or variability, so the pattern of interaction may have changed without a large change in the total percentage. Her proportion of time spent proximate to and interacting 
with the other adult females increased in the new habitat, while it decreased with Mjuvenile.

F-calf (Fig. 11D) spent the majority of her time with her mother in both habitats, but spent less time with her mother and more time with the other adult females in the new habitat. She also showed a decrease in social interaction with M-juvenile (18\% down $4 \%)$, and an increase of social interaction with the adult females combined from $82 \%$ in the previous habitat to $95 \%$ in the new habitat.

M-juvenile (Fig. 11E) interacted with all of the females more evenly in the previous habitat, but was most proximate to Female1. The proportion of time spent proximate to and interacting with the females was more varied in the new habitat, with decreases in time with Female3, and increases with F-calf. Although M-juvenile showed an increase in proportion of time spent with F-calf, she showed a decrease in time with M-juvenile. Inconsistencies in herdmate proximity and social interaction may in part be due to temporary separation of M-juvenile from Female3 and F-calf surrounding estrous periods, so that M-juvenile was not always in the same habitat with all of the females. 


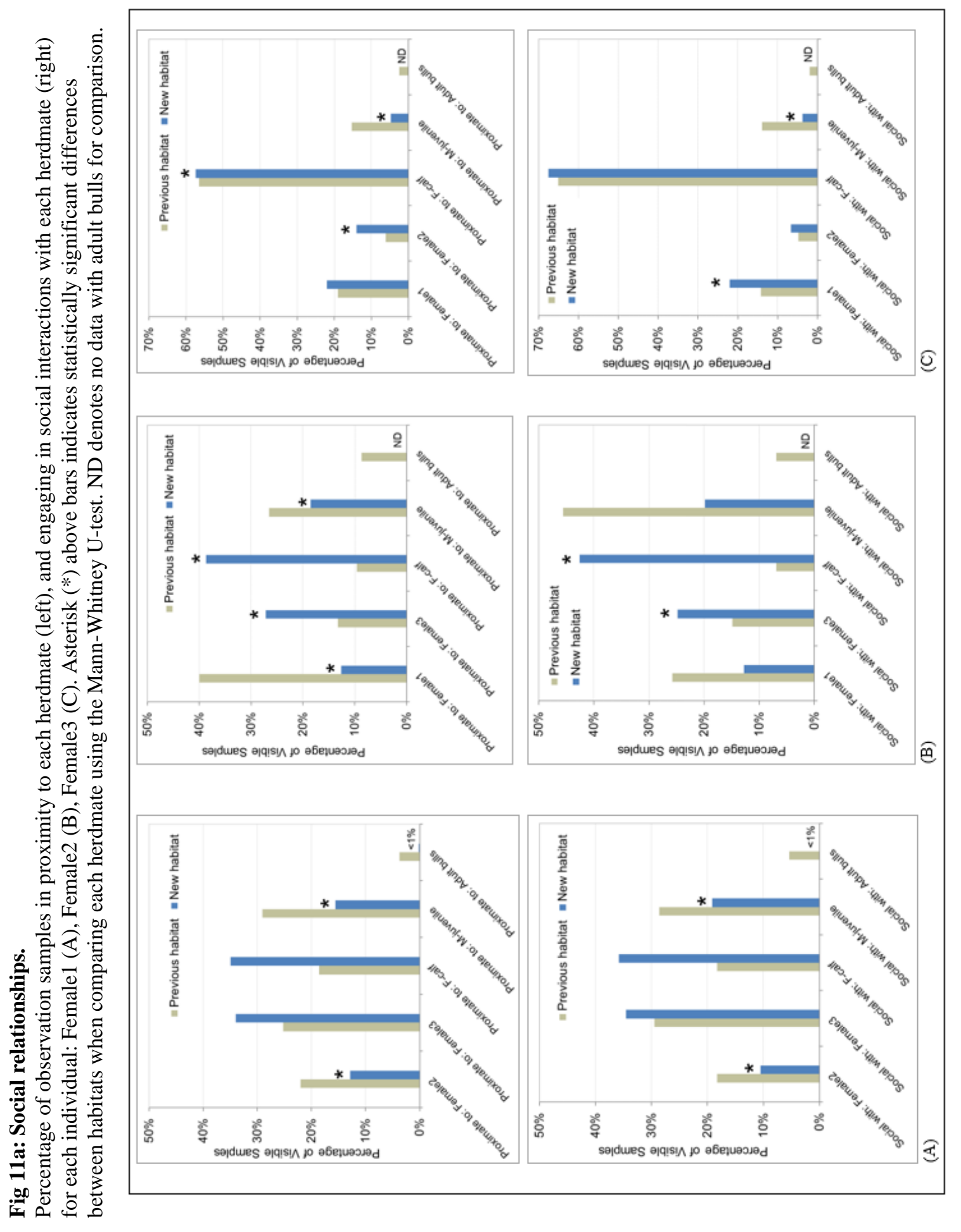



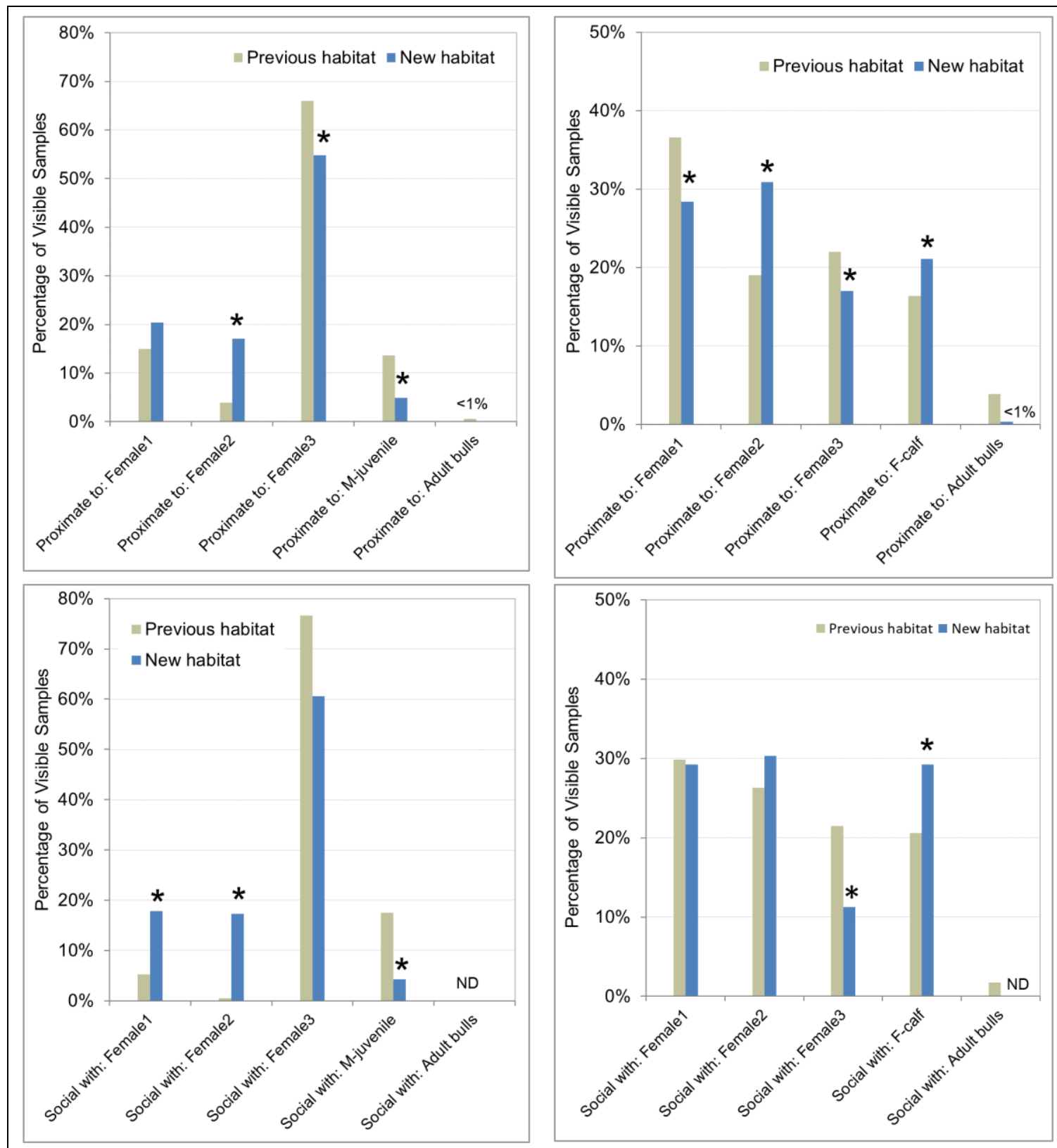

(D)

$(\mathrm{E})$

Fig 11b: Social relationships.

Percentage of observation samples in proximity to each herdmate (left), and engaging in social interactions with each herdmate (right) for each individual: F-calf (D), M-juvenile (E). Asterisk (*) above bars indicates statistically significant differences between habitats when comparing each herdmate using the MannWhitney U-test. ND denotes no data with adult bulls for comparison. 
Affiliative behaviors comprised the majority of all social interactions across all individuals in both habitats (ranged from $72 \%$ to $91 \%$ ) (Fig. 12A). All individuals exhibited a significant change in social partners for affiliative behaviors that mirrored the changes for all social interactions combined (Fig. 11). Chemosensory and reproductive behaviors combined were more frequent in the previous habitat as a result of interactions with adult bulls. Behaviors indicating arousal were observed only in the previous habitat and were rare (less than 1\%). For Female3 and F-calf (Fig. 12A) the slight decrease in time exhibiting dam-calf behaviors of nursing, attempting to nurse, or claiming/protecting of the calf was not significant and were observed only in Female3.

Agonistic or dominant/submissive behaviors increased in the new habitat (from $8 \%$ to 11\%). As focal animals, only Female1, Female2, and M-juvenile exhibited a sufficient proportion of agonistic behaviors for analysis. While habitat differences in the proportion of time engaged in these behaviors were not significant, there were clear senders (instigators) and receivers (recipients), and the proportion of time engaged with each herdmate differed between habitats. Female2 (Fig. 12B) experienced an increased proportion of affiliative behaviors in the new habitat, and a decreased but not significant proportion of agonistic behaviors. Female2 was usually the receiver in agonistic interactions, but this decreased (from $78 \%$ down to $72 \%$ ) in the new habitat. In the previous habitat, Female2 engaged in agonistic interactions most frequently with Mjuvenile (59\%), then Female1 (29\%) and Female3 (18\%). In the new habitat, her agonistic interactions decreased with M-juvenile (down to 28\%) and Female1 (down to $11 \%)$, and she engaged most often with F-calf (33\%) and also showed an increase with 
Female3 (up to 28\%). M-juvenile (Fig. 12C) was usually the instigator in agonistic interactions, and this increased (from $65 \%$ up to $76 \%$ ) in the new habitat, and the proportion of time in which he was mutually engaged as a partner decreased (from $23 \%$ down to $6 \%$ ). In the previous habitat, $M$-juvenile engaged in agonistic interactions most often with Female $2(69 \%)$, then Female3 (18\%), Female1 (9\%) and F-calf $(3 \%)$. In the new habitat, agonistic interactions decreased with Female2 (down to 29\%), increased with Female1 (up to 35\%) and F-calf (up to 18\%), and remained the same with Female3. Female1 (Fig. 12D) was usually the receiver in agonistic interactions in the previous habitat (63\%), but was most often the sender in the previous habitat $(64 \%)$. In the previous habitat, Female1 engaged in agonistic interactions most frequently with Mjuvenile (38\%), then F-calf (25\%), then Female2 (13\%) and Female3 (12\%) \%). In the new habitat, agonistic interactions decreased with M-juvenile (down to 20\%), increased with F-calf who she engaged most with (up to 40\%), and increased with Female2 (up to $20 \%$ ) and Female3 (up to 20\%). 


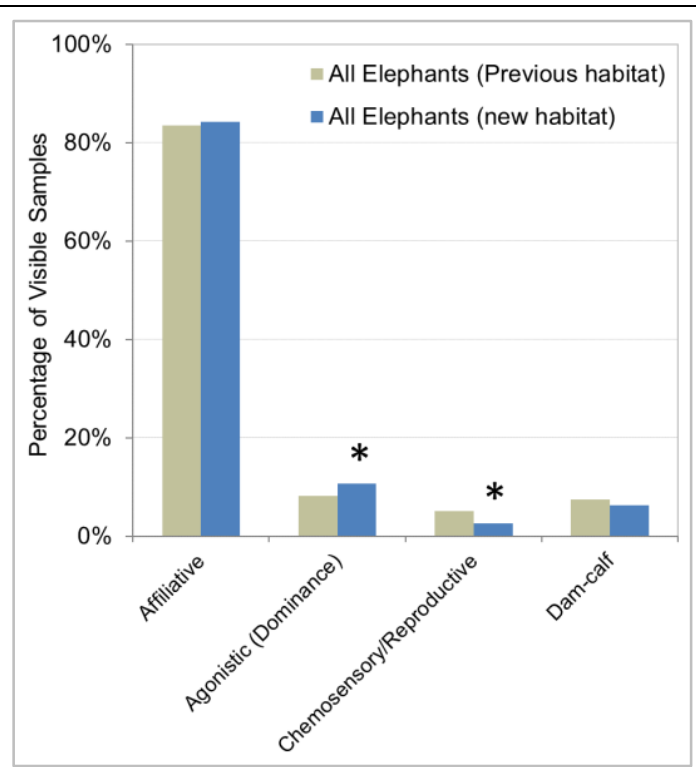

(A)

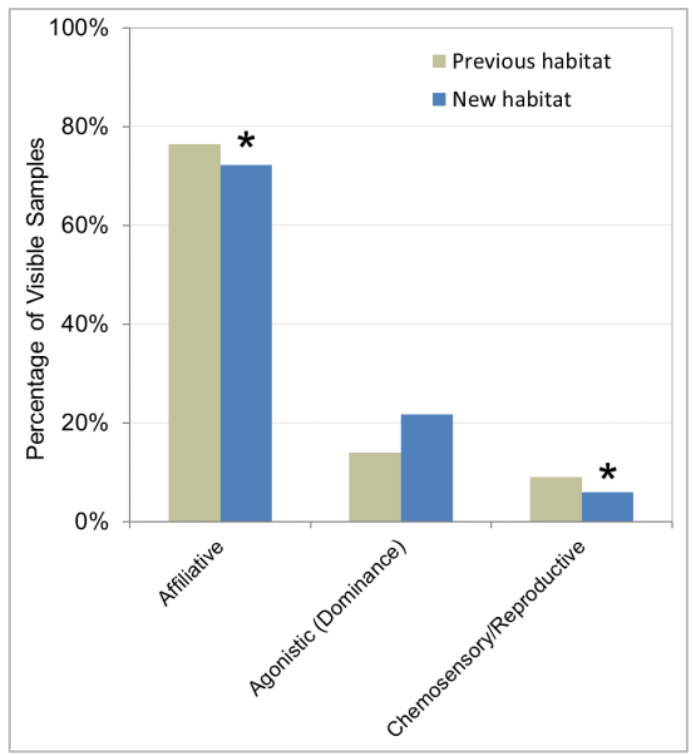

(C)

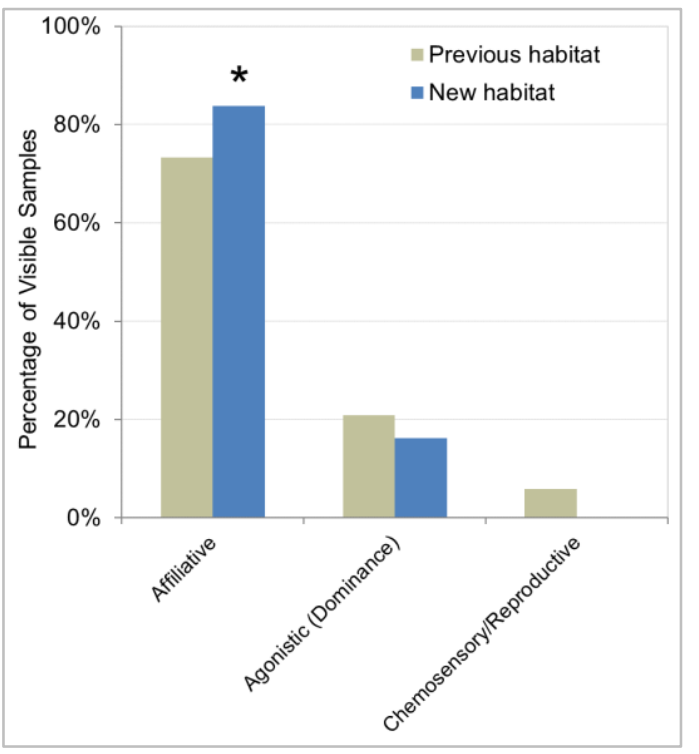

(B)

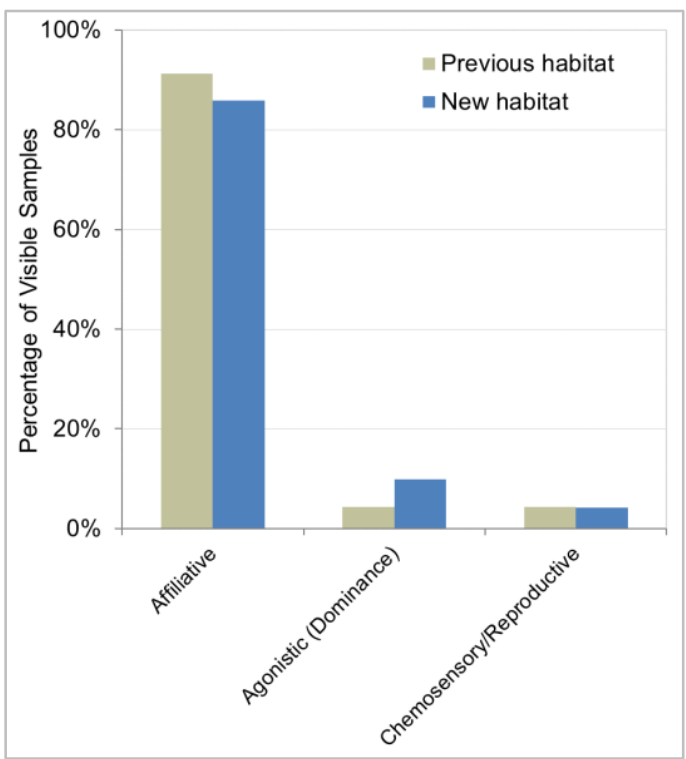

(D)

Fig 12: Types of social behavior.

Percentage of observation samples exhibiting functional types of social behaviors. All elephants (A), Female2 (B), M-juvenile (C), Female1 (D). Asterisk (*) above bars indicates statistically significant differences between habitats when comparing proximity to each herdmate using the Mann-Whitney U-test. 


\section{$\underline{\text { Resource use }}$}

Observed resource use (Fig. 13 and 14) shows the percentage of time allocated to interacting with the diversity of resources available for elephants relative to all interactions with food-delivery resources, habitat features, and enrichment objects.

Relative time spent interacting with resources in the new habitat (Fig. 14) reflects the switch to foraging and eating food distributed in various ways. Interactions with fooddelivery resources increased from $31.5 \%$ in the previous habitat to $83.0 \%$ in the new habitat. Behavior measures showed a decrease in interactions with permanent habitat features, and use of these habitat features relative to other resources decreased from $54.0 \%$ in the previous habitat to $12.1 \%$ in the new habit. Behavior measures showed no significant change in the percentage of time spent interacting with keeper-provided enrichment objects, but with the switch to feeding primarily from food-delivery resources, the use of enrichment objects relative to other resources decreased from $13.1 \%$ in the previous habitat to $4.9 \%$ in the new habit.

The proportion of time spent seeking food and feeding is much higher (34\% higher) than it is for interacting with enrichment objects and habitat features in the new habitat; therefore, the engagement with enrichment objects and habitat features is more rare in the new habitat, and with our sampling methods of recording behavior one day per week with eight 2-minute recording sessions, both the interaction behaviors and overall resource usage may have been underestimated even though the sampling interval of 30-seconds captured more rare behaviors during the initial test period. It is also possible that the 
moving of objects to obtain food (e.g., moving a log under a hanging feeder) was underestimated given the hierarchy of the behavior ethogram. Elephants have also been observed engaging with the inside "rainfall" of the deluge system and the outdoor water cannon; however, these habitat features were not evaluated because they require keeper interaction and were therefore not included in the overall resource analysis.

Interacting with all of these resources in a social context (i.e., sharing the resource) comprised $22.9 \%$ of the habitat resource interactions in the new habitat compared to $10.5 \%$ in the previous habitat. Sharing of food from, or sharing space around a fooddelivery resource comprised $25.9 \%$ of all food-object interactions; simultaneously interacting with the same enrichment object comprised $8.6 \%$ of all enrichment-object interactions; and using the same habitat feature in a social context (e.g., playing in the pool or on logs) comprised $8.0 \%$ of all habitat feature interactions. 


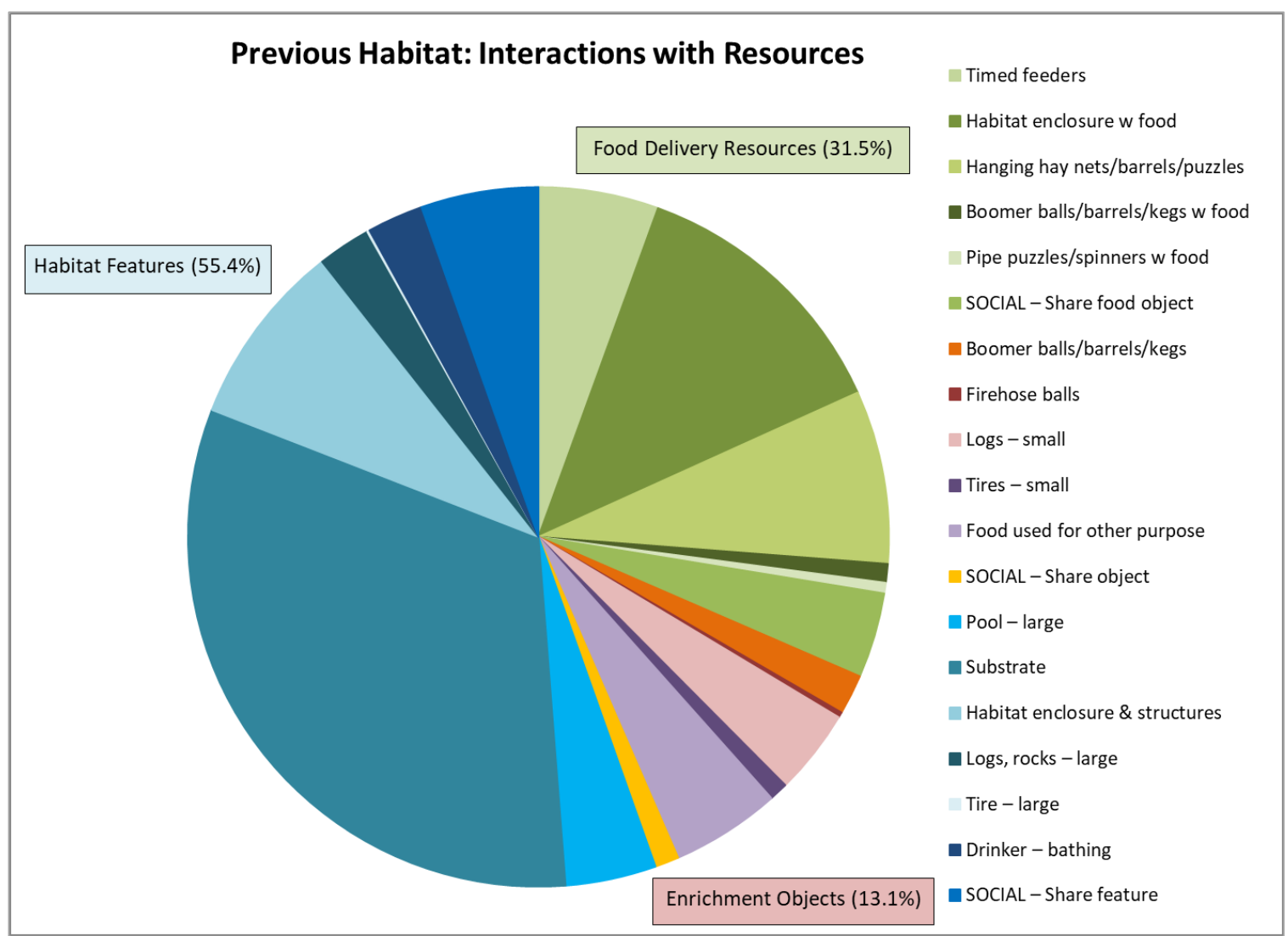

Fig 13: Interactions with resources in the previous habitat. 


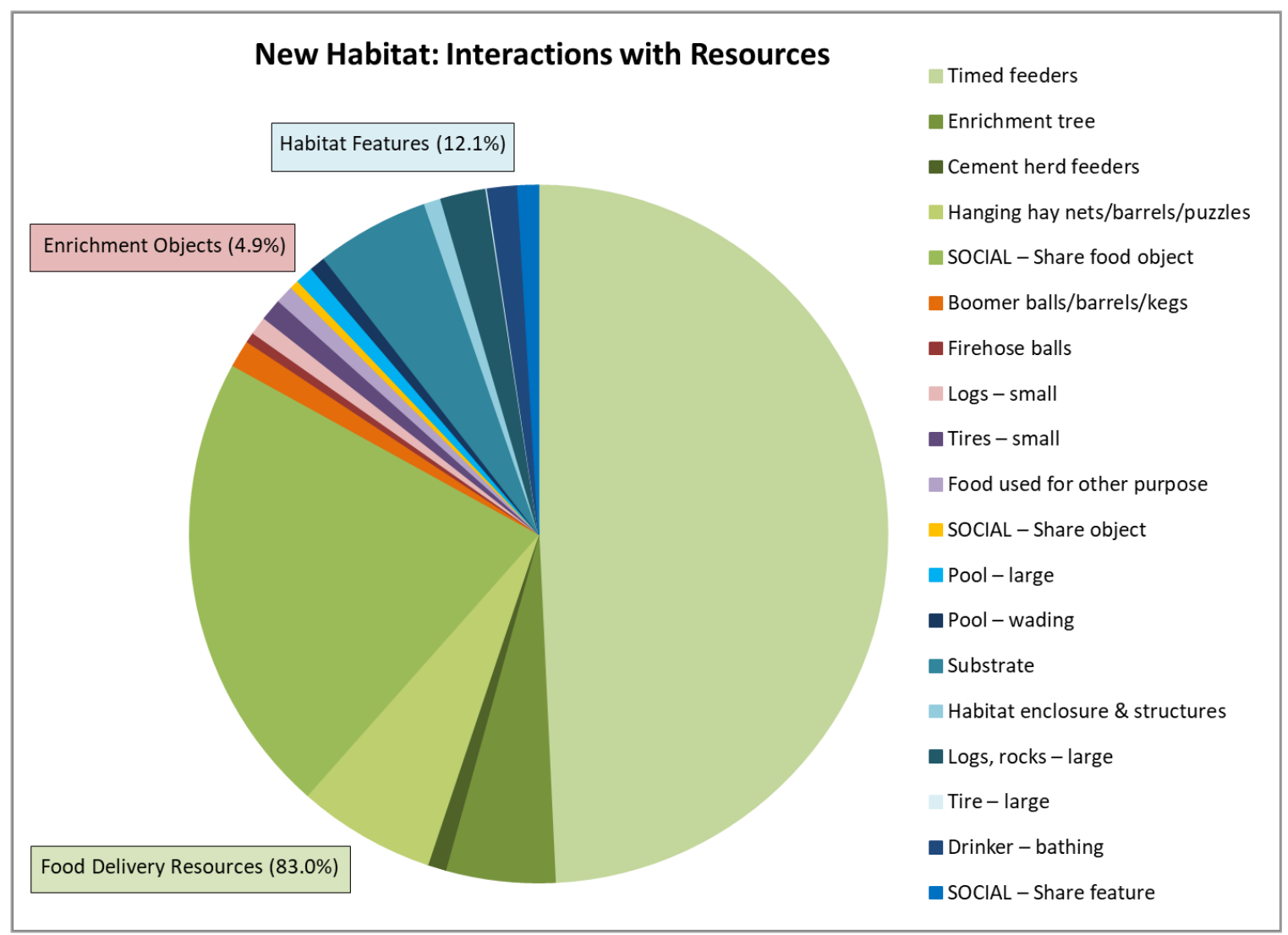

Fig 14: Interactions with resources in the new habitat. 


\section{Discussion}

\section{$\underline{\text { Walking distance }}$}

Movement patterns of wild elephants have been measured under different environmental conditions using a variety of techniques, from radio and GPS collaring to observers following individuals and herds (reviewed in Leighty et al. (2009)). Reported daily walking distances of wild African elephants are generally greater than for Asian elephants. Wild African elephants are reported to walk 3 to $12 \mathrm{~km}$ (1.9 to $7.5 \mathrm{mi})$ per day under normal environmental conditions (Merz, 1986; Theuerkauf and Ellenberg, 2000; Whitehouse and Schoeman, 2003; Slotow and van Dyk, 2004), and can range up to 27 $\mathrm{km}$ (17 mi) per day or more for landscape-level movements such as seasonal migrations or dispersal (Wall et al., 2013) or under extreme conditions such as in water scarce regions of Namibia (Viljoen, 1989; Spinage, 1994; Hertel et al., 2020). By contrast, wild Asian elephants in Myanmar were shown to typically walk less than $1.6 \mathrm{~km}$ to $6.4 \mathrm{~km}$ (1 to $4 \mathrm{mi}$ ) per day, and their home range size varied depending on quantity and quality of food, and whether or not the area was occupied by bulls or by cows and their offspring (Songer et al., 2018). In Sri Lanka, Kurt (1974), as cited in Leighty et al. (2009), also found shorter daily walking distances in wild Asian elephant groups with nursing calves (1 to $3.5 \mathrm{~km}$ ) or juveniles ( 3 to $8.5 \mathrm{~km}$ ). Another measurement of movement is distance displaced, which measures a straight-line change in location as a result of walking any pattern. By measuring displacement, Alfred et al. (2012) found that Bornean elephant herds travelled an average of less than $2 \mathrm{~km}$ per day with a maximum of $4 \mathrm{~km}$ per day, and that daily distance travelled was lower in continuous forest compared to fragmented 
forests in which food and water availability is reduced. It is important to note that methodology for measuring movement in free-ranging animals is often based on relatively low sampling rates to preserve battery power or because of observer limitations, which likely results in an underestimate of total distance walked.

Holdgate et al. (2016) found that adult female African and Asian zoo elephants walked between $1.2 \mathrm{~km}$ and $17.3 \mathrm{~km}(0.75$ and $10.7 \mathrm{mi})$ per day with an average of $5.3 \mathrm{~km}(3.3$ mi), comparable to daily distances of wild elephants under normal conditions; and other studies of zoo elephants have also measured walking distances comparable to wild elephants (reviewed in Rowell (2014). For Female2, bouts of locomotor stereotypy could have inflated measurements of distance walked; however, her repetitive behaviors decreased in the new habitat, and when the percentage of time spent performing repetitive behaviors was removed from walking distance calculations, her mean walking distance would still shows an increase from $15.4 \mathrm{~km}(9.6 \mathrm{mi})$ in the previous habitat to $17.1 \mathrm{~km}$ (10.6 mi) in the new habitat. Holdgate et al. (2016) found individual differences were associated with demographic, social, housing, and feeding-related variables. Interestingly, habitat size overall was not associated with increased walking distance. More diverse feeding regimens correlated with increased walking distance, and elephants that were fed on an unpredictable schedule walked $1.29 \mathrm{~km} /$ day $(0.8 \mathrm{mi})$ further per day than elephants fed on a predictable schedule. One explanation given was that dynamic feeding regimens lead to increased expression of exploratory behavior; which was seen in the current study with elephants checking food delivery resources while moving through the habitat. These findings on the association of feeding regimes and increased walking 
distance support the assessment by Veasey (2020) that foraging is of psychological significance to Asian elephants and that walking may be a largely appetitive behavior synonymous with foraging — that walking in Asian elephants is not a behavior that is expressed for its own sake but rather as a means to secure nutritional, social or physical resources, and as such, walking is largely appetitive.

Based on the data available from studies of wild elephants, the elephants at the Oregon Zoo appear to walk at least as far as, and perhaps at times even farther than their wild counterparts on a daily basis. This may in part be due to the fact that they are secure to move as individuals and their movement is not limited by the group composition or group needs. Wild herds with a nursing calf or old or sick elephants will have to move more slowly; but elephants in a zoo can move as individuals in safety and with their resource needs met.

\section{$\underline{\text { Reproductive and adrenal hormones }}$}

The Oregon Zoo's longitudinal hormone data set provides important baseline information for interpreting hormone results obtained in this study on an individual and group basis. Historically, elephants at the Oregon Zoo have exhibited regular reproductive cycling (Glaeser et al., 2012). The continued and regular cycling observed throughout the construction period and transition to the new habitat represents one indicator of normal reproductive health and welfare for the female elephants in the study. 
In terms of adrenal hormone activity, the return to previous median FGM concentrations in the new habitat, after a period of increased FGM concentrations and variability during the construction time, suggests that the elephants adapted well to their new environment and showed a normal and healthy stress response during the time of change and transition. Furthermore, the elephants were observed closely by their keepers during each transition to any new spaces, and it was determined that all the transitions appeared to be relatively easy and uneventful for this group of elephants. Higher variability in adrenal activity during the construction phase suggests adaptive and normal adrenal responses to life challenges, changes and excitement (e.g., moving out of familiar areas and exploring new areas, learning new feeding strategies and routines, finding different choices in environment and enrichment). The lack of significant differences in variability between the construction phase and previous habitat may be in part due to the small sample size in the previous habitat (i.e., shorter time period of fecal collection) resulting in lower statistical power. Although the females in this study have not exhibited signs of chronic stress (Glaeser et. al. 2020, in review), Carlstead and Brown (2005) argue that high variability in longitudinal FGM data may be a better measure of increased reactivity than increased mean levels, because the best indicator of chronic stress in animals subjected to intense stressors of long duration or frequent repetitiveness may be more frequent and enhanced or exaggerated glucocorticoid responses to new and acutely presented stressors (Mason et al., in prep). However, variability declined significantly in the new habitat, again indicating no evidence of chronic stress. 
Although Female2 did not show a return to previous median levels in the new habitat, visual inspection of charted FGM data did show declines immediately following introduction to new areas which indicates normal adaptive responses, and her social interactions may have been a confounding factor. In the new habitat, Female2 spent more time with M-juvenile than did the other females; and although her agonistic interactions with M-juvenile decreased in the new habitat, courtship and mating-related behaviors were observed by staff, and these interactions can temporarily increase adrenal activity and thus affect the overall median levels (Wielebnowski, 2003) (Glaeser et al., unpublished data). The confounding factor of reproductive state needs to be considered for Female3. Glaeser et al. (2020, in review) showed that serum cortisol covaried with reproductive state in female Asian elephants, and with her first calf, Female3 showed highest mean serum cortisol levels during lactation anestrous, followed by pregnancy, then normal cycling. In the current study, she was in lactational anestrous for the first 34 weeks of 140 weeks in the construction phase (9\% of the samples in this phase), so her reproductive state does not appear to account for the higher median concentrations observed during the construction phase. However, the "stress" of calf-rearing, albeit generally positive in nature, could explain increased variability. Female1 had the largest increase in interquartile range (IQR) during the construction phase compared to other elephants, and she was also the most tentative in exploring new areas and took longer to learn the new feeding strategy. The challenge and change during construction may have resulted in a stronger adrenal response for her based on her temperament and disposition. F-calf's pattern was similar to that observed in the adult females and her concentrations showed intermediate levels compared to the adults. F-calf's first four years of life were 
monitored in this study, and her development stages may have affected her adrenal activity as well, but it is not known whether or how the stress response change with early development in elephants. In a model mammalian species, the rat, Sapolsky and Meaney (1986) found that immature animals had a prolonged stress response compared to adults. Glucocorticoids were secreted in response to stressors in the late fetal period with levels within the range of the adults, followed by a two-week adrenocortical quiescence in the post-natal period, then as the capacity to initiate a stress response emerged with age, the termination of the stress response was delayed compared to adults.

Animals have evolved to cope with environments of great temporal and physical complexity. By contrast, most animals under human care live in environments that are less complex than their natural habitat, and reduced environmental complexity is generally associated with increases in predictability (Bassett and Buchanan-Smith, 2007). The construction phase had disturbances caused by heavy construction as well as elements of unpredictability with introductions to new habitat areas and changes to formerly familiar areas. Studies have shown that unpredictable environmental conditions result in elevated concentrations of glucocorticoids in vertebrates (Wingfield, 2005). Overall, the elephants in this study showed adaptive adrenal responses to disturbance and environmental complexity with individual variability in hormone concentrations and response patterns.

In terms of adrenal hormone activity, the return to previous median FGM concentrations in the new habitat, after a period of increased FGM concentrations and variability during 
the construction time, suggests that the elephants adapted well to their new environment and showed a normal and healthy stress response during the time of change and transition.

\section{$\underline{\text { Behavior }}$}

Overall, the elephants exhibited a diverse range of species-typical behaviors and natural social dynamics of a healthy herd in both habitats. Activity budgets differed between the new and previous habitats, with increased activity levels, increased locomotion, increased foraging, and decreased repetitive behaviors. Interestingly, the elephants also exhibited different social choices between the two habitats with changes in whom they primarily associated with and how they interacted with herd members.

In evaluating the behavioral repertoire of this group of elephants, it is important to define "naturalness" in terms of similarity to their wild counterparts in order to identify desirable species-typical behaviors for a group of animals. Yeates (2018) argues that natural behavior means human intervention has had zero or negligible effect, which is generally not the case in captive or confined environments. However, if a behavior occurs in captivity that is also expressed in the wild, then the behavior is indeed natural.

Furthermore, Veasey et al. (1996) suggest that comparisons with wild animals should be used in combination with other indicators to demonstrate that the consequence of not performing 'wild' behaviors or performing them at a different rate results in compromised welfare; with the reasoning that expression of these behaviors in the wild does not eliminate the possibility that the animal is suffering. The health status of animals 
in modern zoos and their wild conspecifics may differ given the medical care and husbandry of captive animals versus wild animals that face disease and parasitism, untreated injury, and natural mortality. Furthermore, time budgets of zoo animals will likely differ from wild counterparts facing health challenges as well as the pressures of food acquisition, predation, territorial aggression, etc. (Veasey, 2006). The behaviors measured in this study include behaviors expressed in the wild for a group of elephants of mixed ages and both sexes, with additional indicators to determine whether these behaviors are beneficial to an individual.

\section{Activity levels and locomotion}

The increase in percentage of time spent performing active behaviors (i.e., social behaviors, interaction with food-delivery resources, enrichment objects and habitat features, feeding/drinking, and locomotion) indicates that the enriched and more complex environment of the new habitat indeed succeeded in the goal to increase overall activity levels for the elephant herd. Locomotion increased for all individuals but lacked significance for some. However, our behavior data collection methods categorized 'locomotion while feeding' as a feeding behavior; therefore, walking and feeding at the same time (which represented a significant amount of time) was not included in the locomotion behavior category. This likely resulted in an underestimate of locomotion monitored through behavior observation. For Female1, the doubling of measured walking distance via GPS tracking, but the lack of a significant concomitant increase in locomotion as measured through direct behavior observation is therefore likely due to the different sampling methods and frequencies. Behavior observations were conducted only 
during the Zoo's public open hours, and were recorded once a week for 16 minutes with a sampling interval of 30-seconds; whereas GPS data was collected once every two weeks for 24 hours with a sampling interval of 5 seconds. The GPS data is therefore a more reliable representation for overall movement and walking distance.

\section{Foraging, feeding, and exploratory behaviors}

In the previous habitat, keepers provided an array of simple puzzle feeders and used scattered food distribution to extend the duration of feeding and encourage investigation during and after staffed hours. While the average time spent seeking food and eating during staffed hours did not change between the two habitats, our findings indicate that the food-delivery resources designed into the new habitat provided more diverse foraging opportunities and promoted natural foraging behavior for more extended time periods, which was further supported by observed increases in locomotion and measured daily walking distances.

The switch from directly keeper-provided food towards independently seeking food provided in various types feeding devices dispersed widely throughout the new habitat may be providing a superior foraging experience that helps to better satisfy the psychological needs of this species. Foraging and feeding are intrinsically linked, but functionally they are different. Foraging represents the appetitive phase of acquiring nutrients which, for wild elephants involves social interaction, information gathering and processing, group and individual decision making, and locomotion; whereas feeding is the consumption phase and involves less cognition but has more direct survival impact 
(Veasey, 2020). In an assessment of psychological priorities for captive Asian elephant welfare, Veasey (2020) demonstrates the importance of providing species-appropriate feeding, social, and cognitive opportunities; with the greatest priority being appetitive behaviors essential for survival in the wild, followed by behaviors linked to sociality. While providing browse might satisfy specific nutritional requirements in elephants, it is unlikely to satisfy the behavioral or cognitive requirements associated with the act of browsing such as finding the browse, selecting, harvesting and processing browse. Veasey (2020) recommends that wherever possible, animals should be empowered to satisfy their needs by being able to express appropriate motivated behaviors and cognitive processes, rather than have these needs met by direct provisioning through human care takers. In the new habitat at the Oregon Zoo, elephants were able to express motivated behaviors of walking, manipulating objects, climbing, and reaching as part of their new foraging experience. Learning a new feeding regimen requires cognitive processes, and finding food on a daily basis requires locomotion, social interaction, and decision making as to whether to follow the visual signal of another elephant approaching a feeding resource or to check areas on one's own.

\section{Repetitive behaviors}

Repetitive behaviors occurred in the new habitat at a very low rate (average 4\%) and in only two females. A decrease in frequency was found in one female, and a change in bout pattern was observed in the other female, which appeared to be primarily related to feeding anticipation of routine feeding seen in other studies (e.g., elephants (Rees, 2009), polar bears (Montaudouin and Pape, 2005), stabled horses (Cooper et al., 2000), 
carnivores (Carlstead, 1998)). This apparent feeding anticipation subsided as this individual learned to seek food independently rather than directly from keepers, so it's arguable that this behavior served a function. Stereotypies are repetitive behaviors, invariant in form, and with no apparent function (Mason, 1991; Mason and Veasey, 2010). Repetitive behaviors have been well-documented in elephants living in human care (reviewed in Greco et al. (2016)), but their lack of function and inference of current or compromised welfare state have been debated (Mason and Latham, 2004; Mason and Veasey, 2010). The observed decreases in repetitive behavior in this study may be attributed to increased opportunities for expressing species-specific foraging and feeding behavior (Shepherdson et al., 1993; Bashaw et al., 2003; Rees, 2009; Wagman et al., 2018), and other aspects of a more enriched environment (Swaisgood and Shepherdson, 2005; Swaisgood and Shepherdson, 2006), and also partially due to increased time spent with juveniles (Greco et al., 2016) which has been shown to stimulate play or teaching behavior in older herdmates (Garai and Kurt, 2006).

\section{Social behaviors and partners}

Elephants made different choices between the two habitats with respect to who they spent time close to and interacted with, which reflects natural and changing herd dynamics with a young male and female calf transitioning through life stages. Young elephants move away from their nearest neighbours with increasing age to explore their environment (Sukumar, 2003; Gunawardene et al., 2004; Jayantha et al., 2009), which was observed in both M-juvenile and F-calf. 
M-juvenile's decrease in social interactions reflects his changing role in the herd and gradual process becoming more independent from his natal group (Sukumar, 1989; Lee and Moss, 1999). M-juvenile was still a juvenile in the previous habitat, and during the construction phase, at age 6 , exhibited his first signs of musth associated with elevated testosterone. As an adolescent in the new habitat, management necessitated temporary separation from related females during the receptive part of their female reproductive (estrous) cycle to avoid undesirable pregnancies (i.e., inbreeding).

F-calf's changes in proximity and social interaction and her mother's decrease in protective behaviors in the new habitat also reflected natural development changes. In wild elephants, the distance between calves and mothers increases gradually with age (Sukumar, 2003). While nursing infants may stay near their mother all of the time, once they are no-longer milk-dependent, they spend less time in close proximity with their mother (Gunawardene et al., 2004). However, juvenile elephants still spend the highest proportion of their time with their mothers and other adult females (McKay, 1973;

Sukumar, 2003; Jayantha et al., 2009), which was also observed in F-calf. In a study of social relationships in wild juvenile Asian elephants, Jayantha et al. (2009) found that juveniles spent about half their time near their mothers and other adult females, and the majority of remaining time (37\%) near other juveniles. Time spent near sub-adults of either sex was less than $10 \%$, and time spent near adult males was negligible. Social behaviors of juveniles were observed to be most frequent with other juveniles, followed by infants. F-calf also spent the majority of her time near her mother and other adult females in both habitats, but her time close to and engaging with M-juvenile decreased in 
the new habitat as he was maturing into an adolescent. The relative time F-calf spent with each herd mate appeared to be similar to what we would expect in herds with one or two young elephants. Although some changes in herd dynamics were related to temporal factors of sexual and social development, the new habitat provided social opportunities to better support and allow for these changing dynamics.

Not surprisingly, affiliative behaviors comprised the vast majority of social interactions in both habitats. However, the observed agonistic behaviors are important as they serve to establish and reinforce social dominance status, which Schulte (2000) suggests may be important for maintaining social harmony in captivity. Social dominance in elephants is achieved by a combination of size, age, temperament and experience (Sukumar, 1989; Schulte, 2000; Freeman et al., 2004; Archie et al., 2006; Wittemyer et al., 2007), although dominance hierarchies in wild Asian elephant populations in productive habitats have been shown to be weak and nonlinear by comparison to African elephants (de Silva et al., 2016). In our study, M-juvenile was usually the instigator in agonistic interactions, which increased in the new habitat. In the previous habitat, these interactions were primarily with the adult females and mostly with Female2; whereas in the new habitat, the proportion of agonistic interactions with each herdmate was more uniformly distributed and included F-calf, which provides further evidence of M-juvenile's changing role in the herd. In turn, Female2's increase in affiliative behaviors and her decrease in agonistic interactions with M-juvenile and Female1 in the new habitat suggest a more equitable social environment for this individual. Furthermore, the majority of Female2's agonistic interactions in the new habitat were with F-calf, possibly to establish social dominance as 
F-calf had grown to almost the same size. Like Female2, the majority of agonistic interactions in the new habitat for Female1 were with F-calf, likely to discipline or assert dominance.

The presence of adult bulls influences female behavior, which in the previous habitat accounted for courtship and arousal behaviors not observed in the new habitat, and higher proportions of chemosensory and reproductive behaviors. Pinto et al. (2020) found that a group of captive female elephants in the presence of an adult bull exhibited stereotypic behaviors less frequently, spent less time foraging, and more time standing and engaged in courtship behaviors compared to when the bull was absent. However, the percentage of time interacting with the bulls does not account for the lower proportion of time spent foraging and the higher proportion of time stationary in the previous habitat.

Overall decreased proximity among herd members combined with sustained, or increased proportions of time actively engaged in social behaviors implies increased choice of whether to stay near a herdmate in order to engage in a social activity or whether to move away to engage in a different activity. Although providing more opportunities for individual choices was a priority in the design of the new habitat and we observed decreased proximity as a consequence, elephant care staff also created some opportunities for group bonding with large browse feedings that encourage the herd to eat and interact together. 
Inconsistencies in herdmate proximity and interaction between M-juvenile and both Female2 and F-calf when he was the focal observed animal versus when they were the focal animals may be due to temporary separations of M-juvenile from Female3 and Fcalf surrounding estrous. For each focal elephant, the proportion of time spent with each herdmate was influenced by who they had access to at that time; yet we were unable to account for 'access to herd mates' in the data collection and analyses because gates/doors were not always visible to video recorders in order to log this information and observers could not reliably determine 'access to herd mates' from video.

\section{$\underline{\text { Space use }}$}

The layout of spaces in the new habitat allows elephants, when possible, to choose whom to socialize with, giving bulls the opportunity to come in and out of the herd, and for females to stay together as a group or spend time alone, choosing their social partners throughout the day. In addition, the elephants also had increased control over choosing certain environmental conditions with indoor/outdoor access throughout the year, mist/rain stations, and varying substrates.

GPS data showed that the elephants were using the entire area of the new habitat without avoiding any areas or concentrating in any particular space. Although we observed dominance/submissive interactions, including displacement from resources, we did not see evidence of differential space use over time in relation to age or rank in this group of elephants, contrary to findings in some other studies of captive elephants (Leighty et al., 2010) and other herd animals (Robitaille and Prescott, 1993). This equity in resources can 
be attributed in part to food distribution strategies, but also to social dynamics with the majority of social interactions in this group being affiliative. It is important to note that approximately one quarter of the resource interactions in the new habitat were in a social context involving sharing of the resource, with the majority of sharing being related to food.

Elephant care staff have also observed improved foot health and muscle tone in the new habitat as a consequence of the new substrate and of an increased need for various types of body movement to obtain food. Although a previous epidemiological study found that walking distance was not correlated with either foot or musculoskeletal health (Holdgate et al., 2016), for the elephants in our study, walking on natural substrate in the new habitat and getting more daily exercise through increased locomotion across a varied terrain are deemed to be factors important to improving body condition and foot health as indicated by others (Lewis et al., 2010; Miller et al., 2016; Morfeld et al., 2016).

\section{$\underline{\text { Resource use }}$}

Our findings on resource use highlight the value of varied types of food distribution, with timed feeders and overhead feeders being instrumental in encouraging foraging throughout the habit. Of the food distribution resources in the new habitat, all were used extensively with exception of the cement herd feeders, which provided another climbing opportunity and required reaching down to get food. In the timeframe of this study, the feeding holes big enough for adult trunks were also big enough for the calf's foot, so 
holes needed to be plugged temporarily and food was not placed inside to discourage climbing.

Although we observed a decrease in interactions with permanent features in the habitat (e.g., scratching surfaces, large logs), the elephants manipulated their environment more by pulling, pushing, kneeling, stretching, etc. to obtain food items or to move objects for other reasons (including play behavior). It was not uncommon for M-juvenile or the adult females to move a log or stump under an overhead feeder and then stand on it to reach food more easily. The larger and deeper pool also allows for more water-based activity and exercise for all elephants, and the elephants clearly enjoyed swimming and resting in the pool.

\section{$\underline{\text { Conclusion }}$}

Results of this study illustrate that the welfare-based design of the new habitat has indeed achieved desired goals. The elephants are more active, walking farther, and foraging and exploring more; they are expressing a diverse range of species-typical behaviors with changing herd dynamics across life stages; they are exhibiting more control in choosing different aspects of their environment with regular indoor/outdoor access, and they showed extensive resource use. Elephants also experienced biologically meaningful challenges throughout the construction period and subsequent adaptation to changes, and they responded to these challenges in a healthy and adaptive manner. The elephants were found to utilize the entire habitat regularly without avoiding or being excluded from 
areas, suggesting more choice in whether to spend time together or move away to engage in other activities.

With a focus on meeting the physiological, psychological, and social needs of elephants in human care 24-hours a day and through all life stages (Brando and Buchanan-Smith, 2018), new habitats should be designed to support flexibility, allowing for individual choices and self-motivated behaviors day and night and across an individual's entire lifespan. Promoting good welfare focuses on the individual's perspective, and how individuals are affected by varying aspects of life stages, reproductive states, daily and seasonal variations in their environment, as well as ongoing social changes which may affect individual welfare over time. It is important to note that in this study, behavioral evaluations were conducted only during daytime hours, and we relied primarily on hormone measurements and walking distance to assess health and well-being outside of staffed hours. However, physiological and physical health are also assessed regularly by animal care and veterinary staff and the observed improved foot health and muscle tone indicated improved overall health in the new habitat. In addition, the elephants showed normal reproductive and adrenal activity, the physical ability to walk distances comparable to wild counterparts, and the expression of behaviors typical for their life stage. Evaluating psychological welfare is more difficult. Novak and Suomi (1991) generally defined psychological well-being as the ability to adapt and to respond and adjust to changing situations, which was indeed exhibited in this group of elephants. 
One major difference between the environments of captive and wild animals is the reduced amount of environmental control available to them, and the reduced complexity and increased predictability (Carlstead, 1996), which ultimately restricts opportunities to exercise cognitive skills to solve problems and make decisions (Meehan and Mench, 2007). While the dynamic feeding regime introduced unpredictability to encourage foraging, another program goal was to offer the elephants greater control or greater selfdetermination. Bassett and Buchanan-Smith (2007) defined an event as controllable for an animal if the likelihood of the event occurring depends on the behavior of the animal, or if the event occurs as a consequence of the animal's behavior. Buchanan-Smith and Badihi (2012) differentiated choice versus control: choice is the vehicle for perceived control, and allowing choice between alternatives gives an animal control; whereas control is the direct relationship between the animal's behavior and its consequences. Observed choices in sociality and environmental interaction could arguably have provided greater control because the elephants' behavior affected the outcome, but the balance of predictability and control in food acquisition needs further discussion. As a means of survival, animals in a less predictable environment experience higher appetitive motivation for exploration and foraging (reviewed in Bassett and Buchanan-Smith (2007). The new habitat had three types of predictability related to food acquisition temporal predictability, spatial predictability, and signalled predictability. Food was less predictably distributed in time and space in the new habitat, but elephants did learn that if they walked around and checked food sources regularly, they would find food eventually, and by doing so they gained more control and provided more predictability themselves. A reliable signal was the audible sound of a timed feeder tray dropping that could be heard 
if an elephant was close enough, and a signal of varied reliability was the visual signal of another elephant approaching or at a feeding resource. The elephants used cognitive skills in learning a new feeding regimen and assessing signals, and in doing so, expressed more control in the new habitat. The challenge for management becomes that of maintaining the cognitive component in the feeding strategy, in addition to the motor activity (e.g., walking, reaching, manipulation) and extension of feeding time (e.g., timed food disbursement across 14-16 hours per day). Meehan and Mench (2007) argued that appropriate cognitive challenge should also be integrated into foraging enrichment strategies. Next steps in the feeding regime might be to introduce more problem-solving opportunities through signalling or modified puzzles that challenge the elephants in a way matched to their sensory, physical, and cognitive capacities.

The lack of association in previous studies of habitat size to walking distance (Holdgate et al., 2016) or activity level (Scott and LaDue, 2019) challenges the assumption that elephants will choose to walk greater distances if provided with more room. These findings in combination with associations of dynamic feeding regimes and space complexity with walking distance and activity level suggest that space may be important to the extent that it supports more flexibility in the habitat and greater complexity to allow individuals to choose to spend time together or apart. In the current study, habitat size was also increased compared to the previous habitat, so the relationship between size and walking distance, or activity level, could not be directly tested due to the multitude of other factors involved. 
Our ultimate aim is to provide animals in human care with an environment in which they can experience optimum welfare or wellness. An environment that provides sufficient complexity, choice and control will allow animals to thrive within their own capacity, and to develop abilities to cope with the challenges they may face (Brando and Buchanan-Smith, 2018). Mellor (2016) suggests that environments which provide opportunities for animals to engage in behaviors they find rewarding (e.g., exploration, food acquisition, social bonding, mating, care of young) can generate various forms of comfort, pleasure, interest, confidence, and a sense of control. The new habitat constituted a major shift in habitat management for this group of elephants. Rather than caregivers directly providing the bulk of all feedings, the use of integrated food delivery technology (e.g., timed feeders, mechanisms for overhead feeding, etc.) functioning in a semi-autonomous manner was applied to better satisfy the elephants' appetitive motivations of foraging throughout the day and night. Increased spatial complexity, improved spatial features (e.g., large and deep pool), indoor/outdoor access and natural substrates, as well as a multitude of areas to walk to and explore, all helped to create a more beneficial environment to 'set the stage' for improved welfare. Defining and ensuring psychological welfare of elephants in human care might arguably be more challenging than meeting their physiological and physical needs for good welfare. Findings indicate that the complexity and flexibility of the new habitat and habitat management has been effective in improving overall welfare by providing meaningful challenges and the opportunity to experience appetitive motivations and express appetitive behaviors, by offering choice in environmental conditions, and by providing 
the space and resource distribution to support social equity for individuals and changing herd dynamics.

\section{Acknowledgements}

We would like to extend our gratitude to the Oregon Zoo and Oregon Zoo Foundation for funding this research. Thank you to Oregon Zoo elephant manager, Bob Lee, for project support and to elephant care staff, Tarah Bedrossian, Dimas Dominguez, Shawn Finnell, Gilbert Gomez, Jason Miles, Joe Sabastiani, and Pam Starkey, for sample collection and facilitation. Coordination of behavioral video recording during construction depended on regular communication with Zoo Bond construction manager, Jim Mitchell. Thank you to Oregon Zoo endocrine lab manager, Dr. Candace Scarlata, for measuring reproductive hormones; and to Dr. Janine Brown and the Smithsonian's Conservation Biological Institute (SCBI) endocrinology lab for guidance and assistance in measuring adrenal hormones. We appreciate the assistance from Dr. Matthew Holdgate in defining GIS data processing instructions, and from GIS experts with the SCBI Conservation Ecology

Center in solving imagery issues. Much thanks to Ray Losli for his ingenuity in fabricating improved GPS bracelets. We owe immense gratitude to our dedicated team of Oregon Zoo volunteers who gave over 2000 hours to this project: Barbara Lester, Bill Ramirez, Dona Miesen, Jeanne Butcher, Julie Coe, Kathy Street, Kathy Bartholemy, Linda Segal, Marty Clancy, Patti Villareal, Renee Peden, Sarah Friedel, Wayne Schweinfest, Wendy Doerner. We want to thank Oregon Zoo Director Dr. Don Moore and Deputy Directory Sheri Horiszny for their dedication to animal welfare, and to the marketing team for their and enthusiasm for sharing this research with the broader 
community. Finally, the Oregon Zoo is immensely grateful for community support, and

for helping advance the welfare of the elephants entrusted to its care.

\section{Literature Cited}

Alfred, R., A. H. Ahmad, J. Payne, C. Williams, L. N. Ambu, P. M. How, and B. Goossens. 2012. Home Range and Ranging Behaviour of Bornean Elephant (Elephas maximus borneensis) Females. PLoS One 7(2):e31400. doi: 10.1371/journal.pone. 0031400

Archie, E. A., T. A. Morrison, C. A. H. Foley, C. J. Moss, and S. C. Alberts. 2006. Dominance rank relationships among wild female African elephants, Loxodonta africana. Anim Behav 71(1):117-127.

Bashaw, M. J., M. A. Bloomsmith, M. J. Marr, and T. L. Maple. 2003. To hunt or not to hunt? A feeding enrichment experiment with captive large felids. Zoo Biology 22(2):189-198. doi: 10.1002/zoo.10065

Bassett, L., and H. M. Buchanan-Smith. 2007. Effects of predictability on the welfare of captive animals. Applied Animal Behaviour Science 102(3):223-245. doi: https://doi.org/10.1016/j.applanim.2006.05.029

Benjamini, Y., and Y. Hochberg. 1995. Controlling the False Discovery Rate: A Practical and Powerful Approach to Multiple Testing. Journal of the Royal Statistical Society: Series B (Methodological) 57(1):289-300. doi: 10.1111/j.25176161.1995.tb02031.x

Boissy, A., G. Manteuffel, M. B. Jensen, R. O. Moe, B. Spruijt, L. J. Keeling, C. Winckler, B. Forkman, I. Dimitrov, J. Langbein, M. Bakken, I. Veissier, and A. Aubert. 2007. Assessment of positive emotions in animals to improve their welfare. Physiol Behav 92(3):375-397. doi: https://doi.org/10.1016/j.physbeh.2007.02.003

Boult, V. L., R. M. Sibly, T. Quaife, V. Fishlock, C. Moss, and P. C. Lee. 2019. Modelling large herbivore movement decisions: Beyond food availability as a predictor of ranging patterns. African Journal of Ecology 57(1):10-19. doi: 10.1111/aje. 12553 
Brando, S., and H. M. Buchanan-Smith. 2018. The 24/7 approach to promoting optimal welfare for captive wild animals. Behavioural Processes 156:83-95. doi: https://doi.org/10.1016/j.beproc.2017.09.010

Brown, J. L. 2014. Comparative Reproductive Biology of Elephants. In: W. V. Holt, J. L. Brown and P. Comizzoli, editors, Reproductive Sciences in Animal Conservation: Progress and Prospects. Advances in Experimental Medicine and Biology. Springer Science and Business Media, New York, NY. p. 135-169.

Brown, J. L., M. Bush, D. E. Wildt, J. R. Raath, V. d. Vos, and J. G. Howard. 1993. Effects of GnRH analogues on pituitary-testicular function in free-ranging African elephants (Loxodonta africana). Journal of Reproduction and Fertility 99(2):627. doi: 10.1530/jrf.0.0990627

Brown, J. L., K. Carlstead, J. D. Bray, D. Dickey, C. Farin, and K. Ange-van Heugten. 2019. Individual and environmental risk factors associated with fecal glucocorticoid metabolite concentrations in zoo-housed Asian and African elephants. PLoS One 14(9):e0217326. doi: https://doi.org/10.1371/journal.pone.0217326

Brown, J. L., D. C. Kersey, E. W. Freeman, and T. Wagener. 2010. Assessment of diurnal urinary cortisol excretion in Asian and African elephants using different endocrine methods. Zoo Biol 29(2):274-283. doi: https://doi.org/10.1002/zoo.20268

Brown, J. L., and J. Lehnhardt. 1995. Serum and urinary hormones during pregnancy and the peri- and postpartum period in an Asian elephant (Elephas, maximus). Zoo Biol 14(6):555-564. doi: https://doi.org/10.1002/zoo.1430140608

Brown, J. L., and J. Lehnhardt. 1997. Secretory patterns of serum prolactin in Asian (Elephas maximus) and African (Loxodonta africana) elephants during different reproductive states: Comparison with concentrations in a noncycling African elephant. Zoo Biol 16(2):149-159.

Brown, J. L., S. Paris, N. A. Prado-Oviedo, C. L. Meehan, J. N. Hogan, K. A. Morfeld, and K. Carlstead. 2016. Reproductive Health Assessment of Female Elephants in North American Zoos and Association of Husbandry Practices with Reproductive Dysfunction in African Elephants (Loxodonta africana). PLoS One 11(7):e0145673. doi: 10.1371/journal.pone.0145673

Brown, J. L., D. L. Schmitt, A. Bellem, L. H. Graham, and J. Lehnhardt. 1999. Hormone Secretion in the Asian Elephant (Elephas maximus): Characterization of 
Ovulatory and Anovulatory Luteinizing Hormone Surges. Biol Reprod

61(5):1294-1299. doi: https://doi.org/10.1095/biolreprod61.5.1294

Brown, J. L., M. Somerville, H. S. Riddle, M. Keele, C. K. Duer, and E. W. Freeman. 2007. Comparative endocrinology of testicular, adrenal and thyroid function in captive Asian and African elephant bulls. Gen Comp Endocrinol 151(2):153-162. doi: http://dx.doi.org/10.1016/j.ygcen.2007.01.006

Brown, J. L., S. L. Walker, and T. Moeller. 2004. Comparative endocrinology of cycling and non-cycling Asian (Elephas maximus) and African (Loxodonta africana) elephants. Gen Comp Endocrinol 136(3):360-370. doi: https://doi.org/10.1016/j.ygcen.2004.01.013

Brown, M. B., and A. B. Forsythe. 1974. Robust Tests for the Equality of Variances. J Am Stat Assoc 69(346):364-367. doi: https://doi.org/10.1080/01621459.1974.10482955

Buchanan-Smith, H. M., and I. Badihi. 2012. The psychology of control: Effects of control over supplementary light on welfare of marmosets. Appl Anim Behav Sci 137(3):166-174. doi: 10.1016/j.applanim.2011.07.002

Buwalda, B., J. Scholte, S. F. de Boer, C. M. Coppens, and J. M. Koolhaas. 2012. The acute glucocorticoid stress response does not differentiate between rewarding and aversive social stimuli in rats. Horm Behav 61(2):218-226. doi:

https://doi.org/10.1016/j.yhbeh.2011.12.012

Carlstead, K. 1996. Effects of captivity on the behavior of wild mammals. In: D. G. Kleiman, M. E. Allen, Thompson, and L. K.V., S, editors, Wild Mammals in Captivity: Principles and Techniques. University of Chicago Press, Chicago, USA. p. 317-333.

Carlstead, K. 1998. Determining the causes of stereotypic behaviors in zoo carnivores: toward appropriate enrichment strategies. In: D. J. Shepherdson, J. D. Mellen and M. Hutchins, editors, Second nature: Environmental enrichment for captive animals. Smithsonian Institution Press, Washington, D.C. p. 172-183.

Carlstead, K., and J. L. Brown. 2005. Relationships between patterns of Fecal corticoid excretion and behavior, reproduction, and environmental factors in captive black (Diceros bicornis) and white (Ceratotherium simum) rhinoceros. Zoo Biology 24(3):215-232. doi: 10.1002/zoo.20050

Clark, F. 2017. Cognitive enrichment and welfare: Current approaches and future directions. Animal Behavior and Cognition 4(1):52-71. 
Cooper, J. J., L. McDonald, and D. S. Mills. 2000. The effect of increasing visual horizons on stereotypic weaving: implications for the social housing of stabled horses. Applied Animal Behaviour Science 69(1):67-83. doi: https://doi.org/10.1016/S0168-1591(00)00115-5

Cronin, K. A., and S. R. Ross. 2019. Technical Contribution: A Cautionary Note on the Use of Behavioural Diversity (H-Index) in Animal Welfare Science. Animal Welfare 28(2):157-164. doi: 10.7120/09627286.28.2.157

de Silva, S., V. Schmid, and G. Wittemyer. 2016. Fission-fusion processes weaken dominance networks of female Asian elephants in a productive habitat. Behavioral Ecology:arw 153.

Eisenberg, J. F., C. M. McKay, and J. Seidensticker. 1990. Asian Elephants. Smithsonian Institution

Evans, K. E., and S. Harris. 2008. Adolescence in male African elephants, Loxodonta africana, and the importance of sociality. Anim Behav 76(3):779-787.

Fowler, J., L. Cohen, and P. Jarvis. 1999. Practical statistics for field biology. 2nd ed. John Wiley \& Sons.

Freeman, E. W., E. Weiss, and J. L. Brown. 2004. Examination of the interrelationships of behavior, dominance status, and ovarian activity in captive Asian and African elephants. Zoo Biol 23(5):431-448.

Garai, M., and F. Kurt. 2006. The importance of socialisation to the well being of elephants. Zeitschrift des Kölner Zoo 2(49):85-102.

Glaeser, S. S., K. E. Hunt, M. S. Martin, M. Finnegan, and J. L. Brown. 2012. Investigation of individual and group variability in estrous cycle characteristics in female Asian elephants (Elephas maximus) at the Oregon Zoo. Theriogenology 78(2):285-296. doi: http://dx.doi.org/10.1016/j.theriogenology.2012.01.014

Greco, B. J., C. L. Meehan, J. N. Hogan, K. A. Leighty, J. Mellen, G. J. Mason, and J. A. Mench. 2016. The Days and Nights of Zoo Elephants: Using Epidemiology to Better Understand Stereotypic Behavior of African Elephants (Loxodonta africana) and Asian Elephants (Elephas maximus) in North American Zoos. PLoS One 11(7):e0144276. doi: 10.1371/journal.pone.0144276 
Gunawardene, M., L. Jayasinghe, H. Janaka, D. Weerakoon, E. Wickramanayake, and P. Fernando. 2004. Social Organization of Elephants in southern Sri Lanka. In: Symposium for Human-Elephant Relationships and Conflicts, Colombo, Sri Lanka

Hertel, A. G., P. T. Niemelä, N. J. Dingemanse, and T. Mueller. 2020. A guide for studying among-individual behavioral variation from movement data in the wild. Movement Ecology 8(1):30. doi: 10.1186/s40462-020-00216-8

Hines, W. G., and R. J. Hines. 2000. Increased power with modified forms of the Levene (Med) test for heterogeneity of variance. Biometrics 56(2):451-454. doi: 10.1111/j.0006-341x.2000.00451.x

Holdgate, M. R., C. L. Meehan, J. N. Hogan, L. J. Miller, J. Soltis, J. Andrews, and D. J. Shepherdson. 2016. Walking Behavior of Zoo Elephants: Associations between GPS-Measured Daily Walking Distances and Environmental Factors, Social Factors, and Welfare Indicators. PLoS One 11(7):e0150331. doi: 10.1371/journal.pone.0150331

Hutchinson, J. R., D. Schwerda, D. J. Famini, R. H. I. Dale, M. S. Fischer, and R. Kram. 2006. The locomotor kinematics of Asian and African elephants: changes with speed and size. Journal of Experimental Biology 209(19):3812-3827. doi: $10.1242 / \mathrm{jeb} .02443$

Jayantha, D., P. Dayawansa, U. Padmalal, and W. J. J. o. T. T. Ratnasooriya. 2009. Social relationships of wild juvenile Asian Elephants Elephas maximus in the Udawalawa National Park, Sri Lanka. Journal of Threatened Taxa 1(4):211-214.

Kahl, M. P., and C. Santiapillai. 2004. A glossary of elephant terms. Gajah 23:1-36.

Kurt, F. 1974. Remarks on the social structure and ecology of the Ceylon elephant in the Yala National Park. The Behavior of Ungulates and Its Relation to Management:618-634.

Lee, P. C., and C. Moss. 1999. The social context for learning and behavioural development among wild African elephants. In: H. O.Box and Kathleen R. Gibson, editors, Mammallian Social Learning: Comparative and Ecological Perspectives, Zoological Society of London, Symposium 73. Cambridge University Press, Cambridge. p. 102-125.

Leighty, K. A., J. Soltis, and A. Savage. 2010. GPS assessment of the use of exhibit space and resources by African elephants (Loxodonta africana). Zoo Biology 29(2):210-220. 
Leighty, K. A., J. Soltis, C. M. Wesolek, A. Savage, J. Mellen, and J. Lehnhardt. 2009. GPS determination of walking rates in captive African elephants (Loxodonta africana). Zoo Biol 28(1):16-28. doi: doi:10.1002/zoo.20199

Lewis, K. D., D. J. Shepherdson, T. M. Owens, and M. Keele. 2010. A survey of elephant husbandry and foot health in North American zoos. 29(2):221-236. doi: doi:10.1002/zoo.20291

Margulis, S. W., and E. J. Westhus. 2008. Evaluation of different observational sampling regimes for use in zoological parks. Appl Anim Behav Sci 110(3):363-376. doi: https://doi.org/10.1016/j.applanim.2007.05.001

Martin, P., and P. Bateson. 1993. Measuring behaviour: an introductory guide. Cambridge University Press.

Mason, G. J. 1991. Stereotypies: a critical review. Anim. Behav 41(6):1015-1037. doi: https://doi.org/10.1016/S0003-3472(05)80640-2

Mason, G. J., and N. Latham. 2004. Can't stop, won't stop: is stereotypy a reliable animal welfare indicator? Anim Welf

Mason, G. J., and J. S. Veasey. 2010. How should the psychological well-being of zoo elephants be objectively investigated? Zoo Biol 29(2):237-255. doi: 10.1002/zoo.20256

McKay, G. M. 1973. Behavior and ecology of the Asiatic elephant in southeastern Ceylon, Smithsonian Contributions to Zoology No. 125. Smithsonian Institution Press, Washington, DC. p. 1-113.

McPhee, M. E., and K. Carlstead. 2010. The importance of maintaining natural behaviors in captive mammals. In: D. G. Kleiman, K. V. Thompson and C. K. Baer, editors, Wild mammals in captivity: Principles and techniques for zoo management No. 2. University of Chicago Press, Chicago. p. 303-313.

Meehan, C. L., and J. A. Mench. 2007. The challenge of challenge: Can problem solving opportunities enhance animal welfare? Appl Anim Behav Sci 102(3):246-261. doi: 10.1016/j.applanim.2006.05.031

Meehan, C. L., J. A. Mench, K. Carlstead, and J. N. Hogan. 2016. Determining Connections between the Daily Lives of Zoo Elephants and Their Welfare: An Epidemiological Approach. PLoS One 11(7):e0158124. doi: 10.1371/journal.pone.0158124 
Mellor, D. J. 2016. Updating Animal Welfare Thinking: Moving beyond the "Five Freedoms" towards "A Life Worth Living”. Animals 6(3):21.

Mench, J. A. 1998. Environmental enrichment and the importance of exploratory behavior. In: D. J. Shepherdson, J. D. Mellen and M. Hutchins, editors, Second nature: Environmental enrichment for captive animals. Smithsonian Institution Press, Washington DC. p. 30-46.

Merz, G. 1986. Movement patterns and group size of the African forest elephant Loxodonta africana cyclotis in the Tai National Park, Ivory Coast. African Journal of Ecology 24(2):133-136. doi: 10.1111/j.1365-2028.1986.tb00353.x

Miller, M. A., J. N. Hogan, and C. L. Meehan. 2016. Housing and Demographic Risk Factors Impacting Foot and Musculoskeletal Health in African Elephants (Loxodonta africana) and Asian Elephants (Elephas maximus) in North American Zoos. PLoS One 11(7):e0155223. doi: 10.1371/journal.pone.0155223

Montaudouin, S., and G. L. Pape. 2005. Comparison between 28 zoological parks: stereotypic and social behaviours of captive brown bears (Ursus arctos). Applied Animal Behaviour Science 92(1-2):129-141. doi: 10.1016/j.applanim.2004.10.015

Morfeld, K. A., C. L. Meehan, J. N. Hogan, and J. L. Brown. 2016. Assessment of Body Condition in African (Loxodonta africana) and Asian (Elephas maximus) Elephants in North American Zoos and Management Practices Associated with High Body Condition Scores. PLoS One 11(7):e0155146. doi: 10.1371/journal.pone.0155146

Möstl, E., and R. Palme. 2002. Hormones as indicators of stress. Domestic Animal Endocrinology 23(1):67-74. doi: https://doi.org/10.1016/S0739-7240(02)00146-7

Novak, M., and S. Suomi. 1991. Social interaction in nonhuman primates: an underlying theme for primate research. Laboratory Animal Science 41(4):308-314.

Olson, D., editor 2004. Elephant Husbandry Resource Guide. International Elephant Foundation.

Owen, M. A., R. R. Swaisgood, N. M. Czekala, and D. G. Lindburg. 2005. Enclosure choice and well-being in giant pandas: is it all about control? Zoo Biology 24(5):475-481. doi: 10.1002/zoo.20064

Palme, R. 2019. Non-invasive measurement of glucocorticoids: Advances and problems. Physiol Behav 199:229-243. doi: https://doi.org/10.1016/j.physbeh.2018.11.021 
Parra-Frutos, I. 2013. Testing homogeneity of variances with unequal sample sizes. Computational Statistics 28(3):1269-1297. doi: 10.1007/s00180-012-0353-x

Pinto, A., M. Stelvig, C. Costa, J. Colaço, and B. Colaço. 2020. Influence of male presence on female Asian elephants (Elephas maximus) behaviour in captivity. Journal of Zoo and Aquarium Research 8(1):45-49.

Plowman, A. B. 2008. BIAZA statistics guidelines: toward a common application of statistical tests for zoo research. Zoo Biol 27(3):226-233. doi: 10.1002/zoo.20184

R Core Team. 2020. R: A language and environment for statistical computing. $R$ Foundation for Statistical Computing; Available from: https://www.r-project.org/, Vienna, Austria.

Ralph, C. R., and A. J. Tilbrook. 2016. INVITED REVIEW: The usefulness of measuring glucocorticoids for assessing animal welfare. J Anim Sci 94(2):457-470. doi: https://doi.org/10.2527/jas.2015-9645

Rasmussen, L. E. L. 1998. Chemical communication: An integral part of functional Asian elephant (Elephas maximus) society. Écoscience 5(3):410-426. doi: 10.1080/11956860.1998.11682469

Rees, P. A. 2004. Some Preliminary Evidence of the Social Facilitation of Mounting Behavior in a Juvenile Bull Asian Elephant (Elephas maximus). Journal of Applied Animal Welfare Science 7(1):49-58. doi: 10.1207/s15327604jaws0701_3

Rees, P. A. 2009. Activity budgets and the relationship between feeding and stereotypic behaviors in Asian elephants (Elephas maximus) in a Zoo. 28(2):79-97. doi: 10.1002/zoo.20200

Robitaille, J.-F., and J. Prescott. 1993. Use of space and activity budgets in relation to age and social status in a captive herd of American bison, Bison bison. Zoo Biology 12(4):367-379. doi: 10.1002/zoo.1430120407

Rowell, Z. E. 2014. Locomotion in captive Asian elephants (Elephas maximus). J Zoo Aquar Res (4):130-135. doi: 10.19227/jzar.v2i4.50

Sach, F., E. S. Dierenfeld, S. C. Langley-Evans, M. J. Watts, and L. Yon. 2019. African savanna elephants (Loxodonta africana) as an example of a herbivore making movement choices based on nutritional needs. PeerJ 7:e6260. doi: 10.7717/peerj.6260 
Sapolsky, R. M., and M. J. Meaney. 1986. Maturation of the adrenocortical stress response: Neuroendocrine control mechanisms and the stress hyporesponsive period. Brain Research Reviews 11(1):65-76. doi: https://doi.org/10.1016/01650173(86)90010-X

Scarlata, C. D., B. A. Elias, J. R. Godwin, R. A. Powell, D. Shepherdson, L. A. Shipley, and J. L. Brown. 2011. Characterizing gonadal and adrenal activity by fecal steroid analyses in pygmy rabbits (Brachylagus idahoensis). Gen Comp Endocrinol 171(3):373-380. doi: https://doi.org/10.1016/j.ygcen.2011.03.002

Schulte, B. A. 2000. Social structure and helping behavior in captive elephants. Zoo Biol 19(5):447-459.

Scott, N. L., and C. A. LaDue. 2019. The behavioral effects of exhibit size versus complexity in African elephants: A potential solution for smaller spaces. Zoo Biol 38(5):448-457. doi: 10.1002/zoo.21506

Shepherdson, D. 1994. The role of environmental enrichment in the captive breeding and reintroduction of endangered species. In: O. P.J.S., M. G.M. and F. A.T.C., editors, Creative Conservation. Springer. p. 167-177.

Shepherdson, D. 1999. Environmental enrichment for elephants: current status and future directions. Journal of the Elephant Managers Association (JEMA) 10:68-77.

Shepherdson, D. J., K. Carlstead, J. D. Mellen, and J. Seidensticker. 1993. The influence of food presentation on the behavior of small cats in confined environments. Zoo Biol 12(2):203-216. doi: 10.1002/zoo.1430120206

Shepherdson, D. J., J. D. Mellen, and M. Hutchins. 1998. Second Nature: Environmental Enrichment for Captive Animals. Smithsonian Institution Press, Washington, D.C.

Signorell, A., K. Aho, A. Alfons, N. Anderegg, T. Aragon, A. Arppe, A. Baddeley, K. Barton, B. Bolker, and H. Borchers. 2019. DescTools: Tools for descriptive statistics. R package version 0.99. 28. R Foundation for Statistical Computing; Available from: https://www.r-project.org/, Vienna, Austria.

Slade, B. E., B. A. Schulte, and L. E. L. Rasmussen. 2003. Oestrous state dynamics in chemical communication by captive female Asian elephants. Anim Behav 65(4):813-819. 
Slotow, R., and G. van Dyk. 2004. Ranging of older male elephants introduced to an existing small population without older males: Pilanesberg National Park. Koedoe 47(2):14. doi: 10.4102/koedoe.v47i2.82

Songer, M., P. Leimgruber, and J. McEvoy. 2018. Smithsonian's National Zoo and Conservation Biology Institute - Saving Elephants in Myanmar. https://nationalzoo.si.edu/news/saving-elephants-myanmar (Accessed April 12 2018).

Spinage, C. 1994. Elephants. . T \& AD Poyser Ltd., London.

Sukumar, R. 1989. The Asian elephant: ecology and management. Cambridge University Press, United Kingdom.

Sukumar, R. 2003. The living elephants : evolutionary ecology, behavior, and conservation. Oxford University Press, New York.

Swaisgood, R., and D. Shepherdson. 2006. Environmental enrichment as a strategy for mitigating stereotypies in zoo animals: a literature review and meta-analysis. In: G. Mason and J. Rushen, editors, Stereotypic Animal Behaviour: Fundamentals and Applications to Welfare No. 2. p. 256-285.

Swaisgood, R. R., and D. J. Shepherdson. 2005. Scientific approaches to enrichment and stereotypies in zoo animals: what's been done and where should we go next? Zoo Biology 24(6):499-518. doi: 10.1002/zoo.20066

Theuerkauf, J., and H. Ellenberg. 2000. Movements and defaecation of forest elephants in the moist semi-deciduous Bossematié Forest Reserve, Ivory Coast. African Journal of Ecology 38(3):258-261.

Tóthmérész, B. 1995. Comparison of different methods for diversity ordering. Journal of Vegetation Science 6(2):283-290. doi: 10.2307/3236223

Veasey, J. 2006. Concepts in the care and welfare of captive elephants. International Zoo Yearbook 40(1):63-79. doi: 10.1111/j.1748-1090.2006.00063.x

Veasey, J., N. Waran, and R. Young. 1996. On comparing the behaviour of zoo housed animals with wild conspecifics as a welfare indicator. Anim Welf 5:13-24.

Veasey, J. S. 2020. Assessing the Psychological Priorities for Optimising Captive Asian Elephant (Elephas maximus) Welfare. Animals 10(1):39. doi: 10.3390/ani10010039 
Vidya, T. N. C., and R. Sukumar. 2005. Social and reproductive behaviour in elephants. Current Science 89(7):1200-1207.

Viljoen, P. J. 1989. Spatial distribution and movements of elephants (Loxodonta africana) in the northern Namib Desert region of the Kaokoveld, South West Africa/Namibia. Journal of Zoology 219(1):1-19. doi: 10.1111/j.14697998.1989.tb02561.x

Wagman, J. D., K. E. Lukas, P. M. Dennis, M. A. Willis, J. Carroscia, C. Gindlesperger, and M. W. Schook. 2018. A work-for-food enrichment program increases exploration and decreases stereotypies in four species of bears. Zoo Biol 37(1):315. doi: doi:10.1002/zoo.21391

Wall, J., G. Wittemyer, B. Klinkenberg, V. LeMay, and I. Douglas-Hamilton. 2013. Characterizing properties and drivers of long distance movements by elephants (Loxodonta africana) in the Gourma, Mali. Biol Conserv 157:60-68. doi: https://doi.org/10.1016/j.biocon.2012.07.019

Watson, R., C. Munro, K. L. Edwards, V. Norton, J. L. Brown, and S. L. Walker. 2013. Development of a versatile enzyme immunoassay for non-invasive assessment of glucocorticoid metabolites in a diversity of taxonomic species. Gen Comp Endocrinol 186:16-24. doi: https://doi.org/10.1016/j.ygcen.2013.02.001

Watters, J. V., S. W. Margulis, and S. Atsalis. 2009. Behavioral Monitoring in Zoos and Aquariums: A Tool for Guiding Husbandry and Directing Research. 28(1):35-48. doi: doi:10.1002/zoo.20207

Whitehouse, A. M., and D. S. Schoeman. 2003. Ranging behaviour of elephants within a small, fenced area in Addo Elephant National Park, South Africa. African Zoology 38(1):95-108. doi: 10.1080/15627020.2003.11657197

Whitham, J. C., and N. Wielebnowski. 2013. New directions for zoo animal welfare science. Appl Anim Behav Sci 147(3):247-260. doi: https://doi.org/10.1016/j.applanim.2013.02.004

Wielebnowski, N. 2003. Stress and distress: evaluating their impact for the well-being of zoo animals. Journal of the American Veterinary Medical Association 223(7):973-977. doi: 10.2460/javma.2003.223.973

Wielebnowski, N. C., N. Fletchall, K. Carlstead, J. M. Busso, and J. L. Brown. 2002a. Noninvasive assessment of adrenal activity associated with husbandry and behavioral factors in the North American clouded leopard population. Zoo Biol 21(1):77-98. 
Wielebnowski, N. C., K. Ziegler, D. E. Wildt, J. Lukas, and J. L. Brown. 2002b. Impact of social management on reproductive, adrenal and behavioural activity in the cheetah (Acinonyx jubatus). In: Animal Conservation forum. p 291-301.

Wingfield, J. C. 2005. The Concept of Allostasis: Coping With a Capricious Environment. Journal of Mammalogy 86(2):248-254. doi: 10.1644/BHE-004.1 $\%$ J Journal of Mammalogy

Wittemyer, G., W. M. Getz, F. Vollrath, and I. Douglas-Hamilton. 2007. Social dominance, seasonal movements, and spatial segregation in African elephants: a contribution to conservation behavior. Behav. Ecol. Sociobiol. 61(12):1919-1931. doi: 10.1007/s00265-007-0432-0

Yeates, J. 2018. Naturalness and Animal Welfare. Animals (Basel) 8(4):53. doi: 10.3390/ani8040053 


\section{CHAPTER 5: Discussion}

Elephants experience many physiological, social, and environmental changes as part of their natural history and individual life experience; and measures of both positive and negative states are needed to assess the impact of these changes at the individual and group level to help us better understand how animals cope with a changing environment, and to inform management decisions..

An understanding of normal variation of any biomarker is useful in determining how patterns should be taken into account when identifying research questions, planning sample collection, and interpreting results for short- and long-term studies of free-ranging and captive animals. Longitudinal hormone studies in this dissertation highlight the importance of taking intrinsic patterns of hormone secretion into account when evaluating the impact of environmental change on endocrine activity. Through longitudinal assessments of gonadal and adrenal hormones, we confirmed the presence of intrinsic glucocorticoid (GC) patterns associated with reproductive state in both female and male Asian elephants, and provided further evidence that GCs play a role in normal reproductive function.

Animals under professional human care often live beyond the average lifespan of their wild counterparts, and longitudinal studies in aging individuals can provide a valuable contribution to our understanding of reproductive aging and gerontology in wildlife species. Age-related changes in circulating cortisol were observed in both females and 
males, and some individuals experienced higher rates of change in cortisol in one phase of their reproductive cycle versus the other (e.g., higher rates of change in the follicular phase versus the luteal phase, or in musth versus inter-musth periods).

Concentrations of GCs covaried more consistently with physiological changes than with social life events (births, deaths, facility transfers) in females and males. Overall, the elephants in this study showed adaptive adrenal responses to social disturbance and environmental complexity, with individual variability in hormone concentrations and response patterns. Indivduality in adrenal GC response to social life events reinforces the need to assess welfare on an individual basis, and to consider factors influencing the impact of perceived stressors.

With a focus on meeting the physiological, psychological, and social needs of elephants in human care for 24-hours a day and throughout all life stages, new habitats should be designed to support flexibility, allowing for individual choices and self-motivated behaviors. Outcomes from the habitat evaluation and elephant welfare study offer insight on habitat features that promote optimum welfare, most significantly, the dynamic fooddelivery resources, space and resource distribution, varied environmental conditions, natural substrates, topographical complexity, and features of the physical environment that encourage activity and exploration such as the pool and various structures added for exercise opportunities (e.g., large tree trunks, mud wallows, sand piles). 


\section{Intrinsic hormone patterns}

In females (Chapter 2), circulating cortisol was highest during the follicular phase of the ovarian cycle as was found by Fanson et al. (2014); and highest during lactational anestrous compared to all other reproductive states. We see similar patterns in females of other taxa as well. For example. stimulatory effects of GCs on reproductive function have been found in a model rat species (Atkinson and Waddell, 1997) and in a growing number of wildlife and domesticated species [e.g., giant panda (Ailuropoda melanoleuca) (Kersey et al., 2011); musk shrew (Suncus murinus) (Schiml and Rissman, 1999); sheep (Sosa et al., 2013)]. From a metabolic perspective, pregnancy and lactation are energetically demanding due to the increased requirement for nutrients such as glucose. Similar to the spotted hyena (Crocuta Crocuta) (Goymann et al., 2001) and dairy ewe (Pesántez-Pacheco et al., 2019), increased GCs during lactation have also been found in free-ranging Asian elephants (Pokharel et al., 2019), providing evidence that milk production and/or rearing a calf is physiologically and/or physically taxing in elephants.

In bulls (Chapter 3), circulating cortisol covaried positively with testosterone and musth, as observed in previous studies of elephants (Brown et al., 2007; Yon et al., 2007; Chave et al., 2019), and during the analogous condition of rut in other ungulates (Liptrap and Raeside, 1968; Liptrap and Raeside, 1978; Howland et al., 1985; Borg et al., 1991; Barboza et al., 2004; Khonmee et al., 2014). Further studies are needed to elucidate how adrenal GC activity is altered in relation to the physiological changes associated with musth, and whether observed increases in circulating cortisol are due to the energetic 
demands of musth, the challenge of ecological stress during musth, the high metabolic demand and reduced food intake, or a combination of these factors.

\section{Age-related hormone changes}

Age-related changes in circulating cortisol were observed in both females and males (Chapters 2 and 3). Age is confounded with reproductive state in individuals transitioning through life stages, so measurements of age-related changes in hormones should be limited to periods of normal reproductive cycling. Interestingly, some individuals experienced higher rates of change in cortisol in one phase of their reproductive cycle versus the other (e.g., higher rates of change in the follicular phase versus the luteal phase, or in musth versus inter-musth periods).

Within individual females (Chapter 2), circulating cortisol increased with age in 5 of 7 individuals, decreased in the oldest female, and showed no change in the other. A trend towards higher rates of change in cortisol in the follicular phase in the youngest females versus higher rates in the luteal phase in the oldest females suggests that the relationship between cortisol and ovarian activity becomes stronger in the early post-pubertal years. Across cycling females, cortisol increased overall with age, but remained fairly steady across the 21-60 age groups.

Within individual bulls (Chapter 3), age-related changes in cortisol indicated that the extent of increase during musth was changing over time; and the rate of change in cortisol concentrations over time was different between musth and inter-musth periods. 
The age-related increase during inter-musth periods in the youngest male could be associated with his joint issues (Brown et al., 2019; Edwards et al., 2019b). The three older bulls exhibited the greatest magnitude of change during musth; however, the direction of change differed across bulls, which could be related to social factors, musth characteristics, or individual differences. An overall increase in cortisol with age across bulls agreed with previous studies (Brown et al., 2007; Chave et al., 2019).

The association between cortisol and age is complex, as highlighted by dissimilar patterns across individuals of similar age. Bansiddhi et al. (2019) suggests the age effects on the hypothalamic-pituitary-adrenal (HPA) axis are confounded by factors that depend on the environments in which the elephants live. In males, further studies are needed to explain age-related differences across bulls, and whether any of the observed variation could be explained by social dynamics. Further investigation into the relationship between cortisol and ovarian activity, and cortisol and musth may help to clarify the role of cortisol in the transition between reproductive stages (e.g., puberty, reproductive senescence). Reproductive senescence, the decline in reproductive success with advancing age, is ubiquitous in free-ranging populations of vertebrates (Lemaître and Gaillard, 2017). However, evidence of a post-reproductive life stage, an extended stage where females undergo menopause and terminate reproduction, is rare in wild populations of mammals (Ellis et al., 2018). As such, the literature is limited on the physiology of reproductive aging in non-human mammals, especially in males. 
All older bulls (Chapter 3) showed decreased mean testosterone past 31-40-years of age, whereas the youngest bull showed increased mean testosterone with age in his early musth cycles, then a gradual decrease. Thongtip et al. (2008) found age-related decreases in testosterone across Asian elephant bulls during inter-musth periods. Together these findings provide evidence of age-related decreases in testosterone similar to that found in male rats (Wang et al., 1993) and humans (Bremner et al., 1983; Morley et al., 1997). However, across bulls, there were no definitive relationships between higher testosterone concentrations and dominance or age.

Finally, animals under professional human care often live beyond the average lifespan of their wild counterparts, and identifying age-related behavioral, physical, and cognitive changes that may limit positive welfare opportunities can inform husbandry decisions (Watters et al., 2015; Krebs et al., 2018). Longitudinal hormone studies in aging individuals can provide a valuable contribution to our understanding of reproductive aging and gerontology in wildlife species, and may help to differentiate between changes due to normal senescence and those due to pathologies.

\section{Adrenal response to social and environmental change}

Concentrations of GCs covaried more consistently with physiological changes than with social life events (births, deaths, facility transfers) in females and males (Chapters 2 and 3). Because social life events can disrupt stable social groups and social bonds, it was surprising that less than $40 \%$ of social life events altered adrenal GC activity in females, and the adrenal responses were activated only short-term in most cases. However, 
because stimuli that activate the stress response can be positive or negative, a more integrated approach that includes behavioral and other physiological and/or physical measures is necessary to better understand how animals perceive and experience changes and challenges. In the retrospective evaluations of longitudinal hormone analyses spanning many years and using historical records (Chapters 2 and 3), it was not possible to include consistent behavioral data to enhance the implications of our hormone analyses. However, in our evaluation of health and well-being of the elephant herd throughout their transition to a more complex and expanded habitat (Chapter 4), we did include both behavioral and physiological indicators in response to major environmental and husbandry changes - changes that were expected to present many opportunities and meaningful challenges. Overall, the elephants in this study showed adaptive adrenal responses to disturbance and environmental complexity, with individual variability in hormone concentrations and response patterns. Fecal glucocorticoid metabolite (FGM) concentrations and variability were highest during the period of major change with heavy construction and many elements of unpredictability in new and modified habitats and altered feeding regimes and routines. FGM concentrations returned to previous levels once the elephants were completely moved and settled into the new habitat.

The response of females to transfers in and out of males (Chapter 2) reinforces the important role of males in the herd, regardless of their social integration. Pinto et al (2020) concludes that even temporary integration of a male elephant into a female group in captivity has positive impacts in females, and that further studies should be done on the influence of males on welfare of females in captivity. Furthermore, a transfer out of a 
herdmate constitutes a sudden change that is not predictable, with no signals of impending departure (as perceived by resident animals) such as illness or changes in behavior - a change that is not part of the natural history for this species although it may be part of an individual's personal history. Regardless of the social relationships of the transferred individual, a better understanding of the impact on resident animals can inform management of potential ways to help resident animals cope with the change.

Indivduality in adrenal GC response to social life events reinforces the need to assess welfare on an individual basis, and provides evidence that, in addition to intrinsic factors such as genetic and maternal influence affecting the GC response (Cockrem, 2013), an individual's personal history (Prado-Oviedo et al., 2016), personality and temperament or coping styles (Koolhaas et al., 1999), and social relationships and social support (Rault, 2012) are important factors influencing the impact of perceived stressors. A wonderful example of the possible influence of temperament was observed in the oldest female (female F7NZ in Chapter 2). She showed no adrenal response to major social change, and was described by her caretaker as "a very wise elephant who socializes well with both elephants and people. She is careful and does not like change, but copes with it well by letting others experience things first, and then she will follow."

Degree of familiarity and stability of social bonds are factors influencing social support (Rault, 2012), and differences in sociality between African and Asian female elephants suggest we might expect a more pronounced response to social disturbance in female African elephants than what we have observed in Asian elephants (Chapter 2). Brown et 
al. (2019) found that African elephants were more responsive to social stressors than Asian elephants. In the wild, African elephants form complex, multi-tiered social groups that are important to survival, whereas Asian herds are smaller and bonds are more fluid (de Silva and Wittemyer, 2012). At least in parts of their range, Asian elephants exhibit a fission-fusion social structure of familial and non-related individuals, and individuals associate with a pool of long-term companions (Gunawardene et al., 2004; de Silva et al., 2011). By contrast to the accepted view that dominance among African elephant females is age-based, it appears the fission-fusion processes weakens the dominance structure of female Asian elephants in a productive habitat (de Silva et al., 2016). As a reflection of their respective societies, group stability may be more important for African elephants, and choice of partners more important for Asian elephants. Thus, it stands to reason that the observed adrenal response to social disturbance of births, deaths, and transfers of herdmates (Chapter 2) was not as pronounced as we initially had expected.

\section{Supporting welfare and herd dynamics through habitat design}

One major difference between the environments of captive and wild animals is the reduced amount of environmental control available to them, and the reduced complexity and increased predictability (Carlstead, 1996), which ultimately restricts opportunities to exercise cognitive skills to solve problems and make decisions (Meehan and Mench, 2007). Findings in Chapter 4 indicated that the complexity and flexibility of the new habitat at the Oregon Zoo was effective in improving overall welfare by providing meaningful challenges and the opportunity to express appetitive behaviors through feeding enrichment design elements, by offering increased control over environmental 
conditions and social interactions, and by providing the space and resource distribution to support changing herd dynamics and greater social equity of individuals.

In the new habitat, the disbursement of food with less predictability, and food-delivery mechanisms that require elephants to work for food, promoted foraging behavior that more closely mimics that of wild elephants (Spinage, 1994; Sukumar, 2003). Foraging also helps satisfy appetitive motivations important for psychological well-being; and the measured increase in walking distance supports the assessment by Veasey (2020) that walking may be a largely appetitive behavior synonymous with foraging. The provision of foraging opportunities 14 to 16 hours per day, including crepuscular and nocturnal periods, supports the 24/7 approach to promoting animal welfare for this species (Brando and Buchanan-Smith, 2018). Brando and Buchanan-Smith (2018) presented the 24/7 (24 hours and 7 days a week) welfare approach as a framework whereby features of each life stage that are likely to be important to the welfare of the species (e.g., social learning for a juvenile) are accounted for in relation to variations in the captive environment, across day and night, and every day of the year.

While the dynamic feeding regime introduced more unpredictability to encourage foraging, another program goal was to offer the elephants greater control. Providing animals with some level of choice and control within the limitations of their habitat and management routine is regarded to be of high importance for psychological welfare (Buchanan-Smith and Badihi, 2012). Unfortunately, the combined and sometimes interchangeable use of these two terms makes it challenging to apply these concepts and 
evaluate whether an animal is experiencing choice and/or control. Buchanan-Smith and Badihi (2012) presented the following definitions: choice is the vehicle for perceived control, and allowing choice between alternatives gives an animal control; whereas control is the direct relationship between the animal's behaviour and its consequences. Definitions proposed by researchers at the San Diego Zoo are that choice is the opportunity or power to make a decision, while control is an animal's ability to affect the outcome of an event as a result of its behavior. A common framework in psychology is that control of the environment is dependent on the animals behavior; perception of control is more important than actual control, and choice may provide some level of perceived control (Jason Newsom, pers. comm.). In the transition to the new habitat, the elephants learned that if they walked around regularly and checked various food sources distributed across the habitat space, they would eventually find food, and by doing so they gained more control and provided increased predictability for themselves.

In the new habitat, elephants also have increased control of their environmental conditions, with indoor/outdoor access throughout the year, outside covered areas with heaters and misters, and varying substrates. Brown et al. (2019) found that providing increased choice of being indoors or outdoors was associated with a decrease in mean FGM in both Asian and African elephants, but an increase in FGM variability. Based on these findings, the authors speculated that a given individual might benefit from having increased choice and control over its own situation, but it has no control over the whereabouts of the other elephants, potentially resulting in more variable adrenal responses. 
The layout of spaces in the new habitat allows elephants, when possible, to choose their social partners and exhibit greater control over their social interactions. Although we observed dominance/submissive interactions, including displacement from resources, we did not see evidence of differential space use over time in relation to age or rank. This equity in resources can be attributed in part to food distribution strategies, but also to social dynamics in this group of elephants, with the majority of social interactions in this group being affiliative.

The elephants expressed a diverse range of species-typical behaviors and demonstrated social dynamics of a healthy herd, in both the old and new habitats. Elephants made different choices between the habitats in who to spend time with, which reflects natural and changing herd dynamics with a young male and female calf transitioning through life stages (Sukumar, 2003; Gunawardene et al., 2004; Jayantha et al., 2009). With a focus on meeting the physiological, psychological, and social needs of elephants in human care for 24-hours a day and throughout all life stages (Brando and Buchanan-Smith, 2018), new habitats should be designed to support flexibility, allowing for individual choices and self-motivated behaviors day and night and across an individual's entire lifespan.

Outcomes from this habitat evaluation and elephant welfare study (Chapter 4) offer insight on habitat features that promote optimum welfare, most significantly, the dynamic food-delivery resources, space and resource distribution, varied environmental conditions, natural substrates, topographical complexity, and features of the physical 
environment that encourage activity and exploration such as the pool and other structures that promote exercise. The assessment methods used to quantify the effect of habitat and social changes can also provide a model for future investigations of individual and group responses to various transitions — facility transfers, habitat modifications, changes in social structure, rehabilitation and release, translocations — as well as for long-term monitoring of animal welfare.

\section{Conclusion}

In this body of work, we evaluated individual and group response to physiological, social, and environmental changes in zoo-housed Asian elephants, male and female, young and old and across life history stages. These long-term studies of known individuals have helped us gain a better understanding of the physiological states that can affect adrenal GC activity in male and female Asian elephants, and the variation we can expect with physiological changes such as in reproductive cycling, versus variation that is individualistic and related in part to social and environmental factors. This work highlights the importance of taking intrinsic patterns of hormone secretion into account when evaluating the impact of change due to extrinsic factors, especially when informing management decisions. A better understanding of the impact of social change and resiliency to perceived stressors allows us to improve social management of animals, in both the captive setting and free-ranging environments, to enhance welfare.

Findings from the exhibit transition study also offer new insights on the habitat features that help us meet the physiological, psychological and social needs of elephants under 
human care across all life stages, and which of those features may be most beneficial for enhancing welfare of elephants in human care. The long history of Asian elephants under human care, dating as far back as 1000 to $700 \mathrm{BC}$ when elephants first were used in warfare, then later for transportation, logging, and as cultural and religious icons, has resulted in a spectrum of husbandry practices across Asian range countries today. As such, one challenge is to apply welfare priorities across vastly different conditions. Psychological well-being across a variety of conditions could be improved by providing more choices to allow for some level of control or the perception of control. For example, disbursing food with less predictability but in a way that the elephant can create predictability through its own behavior may help to provide some form of control.

Cognitive challenges could be integrated into feeding strategies by providing opportunities to solve problems that challenge the elephants in a way matched to their sensory, physical, and cognitive capacities. Social management should strive to support social equity for individuals and changing herd dynamics across life stages. In addition to our findings on habitat features that encouraged activity and exploration and promoted natural behavior and herd dynamics, Brown et al. (2020) outlined commonalities in how environmental and husbandry factors affect physical and physiological welfare outcomes under the divergent elephant management systems in zoos in North American and tourist camps in Thailand. Consistent with our findings, elephants appeared to have better health and welfare in more enriched environments, with good exercise, and more choices. 
Our newly gained knowledge and deeper understanding of Asian elephant physiology, and of environmental and husbandry factors that can help improve elephant welfare, will be shared not only with the animal management, welfare, and conservation communities to affect change, but also more broadly with the general public to increase understanding of the complexity of elephant needs and to engender empathy for this iconic species. Concerns about animal welfare shape people's perspectives on having animals under human care. Officially accredited zoos (i.e., accredited by AZA, EAZA, or other WAZA approved accreditation body) around the world are regarded as experts in the care and conservation of animals. As welfare experts, we have an opportunity to help our community make evidence-based decisions on animal care programs they support.

\section{Future directions}

The underlying mechanisms through which social behavior, both animal-animal and human-animal interactions, may provide a buffer against stressors could be examined by analyzing samples for oxytocin, immunoglobulin-A, and dehydroepiandrosterone.

Oxytocin is a neuropeptide known for promoting social behavior through which positive social interactions suppress the HPA axis (DeVries et al., 2003) and more generally for modulating affiliative behaviors in both sexes (Ross and Young, 2009). It is possible oxytocin could help clarify the role of prosocial behavior and social support in how individual elephants respond to stressors. $\underline{\operatorname{Immunoglobulin}-\mathrm{A}(\operatorname{IgA})}$ is an immune protein that could provide a broader assessment of individual welfare and help distinguish positive and negative effects (Staley et al., 2018). IgA is downregulated during times of physiological and psychological stress and increase in response to positive stimuli 
[reviewed in Edwards et al. (2019) and Plangsangmas et al. (2020)], rendering it a potential biomarker of both stress and positive affect. However, the circadian rhythm pattern of secretory IgA in Asian elephants (Plangsangmas et al., 2020) presents constraints on sample collection. Dehydroepiandrosterone (DHEA) is an adrenal hormone that might further our understanding of the role of adrenal hormones in reproductive function and of age effects on the HPA axis in both sexes. DHEA has been shown to increase during musth in Asian elephants (Yon et al., 2007) and decrease with advanced age in humans (Samaras et al., 2013). Measuring DHEA across different reproductive states could shed light on additional intrinsic patterns and any covariance with GCs. Age-related changes in DHEA and other adrenal hormones could provide insight into the relationship between adrenal activity and metabolic changes, and aid in the care of geriatric elephants.

Investigations of elephant herd dynamics might benefit from the use of social network analyses, as it does for other social species. As in our findings, Coleing (2009) showed that many facets of individual behaviour in Asian elephants could be understood in terms of social structure of the group. Behavioral assessments to help identify appropriate cognitive challenges for this species could help improve psychological welfare across life stages. Investigations in how choice relates to real or perceived control could help inform habitat design and animal husbandry. Finally, the human-animal relationship is central to the lives of captive animals, and evaluating human-animal interactions, human attitudes, and animal temperament, could help better define an individual's social needs and relationships across life stages. 
Bull management and welfare needs more attention. We need to better meet the psychological needs of bull elephants in the captive environment, with appropriate social development opportunities for young male elephants. Factors influencing social development and transition through puberty in young male elephants (and of young males of other wildlife species) has remained one of my research interests. Bull society is more structured than previously thought, and mature males are important in the learning period of adolescent males (Sukumar, 2003; Rees, 2004; Garai and Kurt, 2006; Evans and Harris, 2008). Social management should support multi-generational matrilineal groups, which bulls can join or leave as they would in free-ranging populations (McKay, 1973). Exposure to other bulls plays a role in the social and physiological development of males into adulthood. In a typical elephant herd, male calves will be exposed to adult males early in life, with their first exposure being when dominant bulls come into the herd to breed with cycling females. As a young bull develops, he learns his place in the hierarchy, and he learns how a breeding male interacts with a herd. In both African and Asian elephants, males disperse from the natal group around puberty and may have loose associations with other bulls and join temporary bachelor groups. Factors in the captive environment that influence the onset of musth also impact the age at which a young male is pushed out of his natal herd. It is important to consider that elephant society in freeranging herds may also be altered due to human influence; therefore, insights we gain on flexibility of male elephant society in captive settings could have conservation implications for wild counterparts. For example, in the Malaysian state of Sabah on Borneo, male elephants have been observed in closer association with each other than 
before, and this has been assumed to be due to increased pressures of habitat loss and fragmentation (Nurzhafarina Othman, pers. comm.). Still, relatively few zoos house multiple males (Hartley et al., 2019; Lee, 2019) to support these known social dynamics. Furthermore, male-male competition may be an important aspect of appetitive courtship behavior that is rarely experienced in captivity, and we need to gain a better understanding of this influence on bull libido for managed breeding. Hartley et al. (2019) outlined broad considerations for social management of male elephants across life stages. To provide a socially and psychologically stimulating environment for captive male elephants throughout their entire life, we must ensure that they can form social bonds and understand their place in the hierarchy in order for them to be moved through a variety of socially compatible situations with other bulls and cows.

With increasing human expansion and habitat decline worldwide, wildlife is experiencing greater degrees of human interaction and varying degrees of management, even in protected areas. Wild Asian elephants are brought into human care as orphans, "conflict animals", and/or as rehabilitation cases. Factors that influence health and well-being of zoo animals are also likely to play a role in wild populations that are intensely managed (Cameron and Ryan, 2016), for example in small reserves or in degraded landscapes. The methods used in this body of work could provide a model for future investigations of individual and group responses to various transitions - facility transfers, habitat modifications, changes in social structure, rehabilitation and release, translocations, rewilding - as well as for long-term monitoring. Global positioning system (GPS) is a technology that has great potential for a broader application to track elephant movement 
and some types of behavioral activity. For example, Hacker et al. (2015) compared GPS data to behavioral data and keeper assessments, and showed that GPS location data can serve as a proxy for assessing social relationships within a group of animals, which would be particularly beneficial in large reserves where regular behavioral observations become more challenging. Future research could use the methodology outlined by Hacker et al. (2015) on a larger and less intensely managed group to assess social relationships.

One of the highest priorities for my future work is to collaborate with field conservation partners to help ensure animal welfare is a principle component of their conservation and husbandry practices. From personal experience through foundation work and involvement in zoo conservation support programs, it is clear that animal health is a priority in range countries, but animal welfare needs greater attention, especially with regards to social management, choice, and cognitive challenge. A low-cost and easy-touse methodology for welfare assessment of elephants under human care is needed. Yon et al. (2019) developed a welfare assessment tool for routine use by elephant keepers, which consists of three parts: qualitative behavior assessment (QBA), daytime behavior questions, and night-time observations. This tool could provide insight into how management practices affect animal welfare on individual and group levels for elephants used in tourism and conservation patrols, and in wildlife parks and zoos. Incorporating this tool in future studies of elephants under different living conditions would be beneficial. For elephants in rescue and rehabilitation situations, it would be beneficial to have welfare assessment tools that utilize more qualitative measures without the need of expensive technology or specialized skills. Specifically, methods for assessing affective 
state through behavioral observation would be beneficial. Finally, developing welldefined strategies for enhancing positive welfare states for elephants across life stages and under a wide range of living conditions could improve care and welfare of elephants worldwide.

\section{Literature Cited}

Atkinson, H. C., and B. J. Waddell. 1997. Circadian variation in basal plasma corticosterone and adrenocorticotropin in the rat: sexual dimorphism and changes across the estrous cycle. Endocrinology 138(9):3842-3848. doi: https://doi.org/10.1210/endo.138.9.5395

Bansiddhi, P., J. L. Brown, J. Khonmee, T. Norkaew, K. Nganvongpanit, V. Punyapornwithaya, T. Angkawanish, C. Somgird, and C. Thitaram. 2019. Management factors affecting adrenal glucocorticoid activity of tourist camp elephants in Thailand and implications for elephant welfare. PLoS One 14(10):e0221537. doi: 10.1371/journal.pone.0221537

Barboza, P. S., D. W. Hartbauer, W. E. Hauer, and J. E. Blake. 2004. Polygynous mating impairs body condition and homeostasis in male reindeer (Rangifer tarandus tarandus). J Comp Physiol B 174(4):309-317. doi: 10.1007/s00360-004-0416-6

Borg, K. E., K. L. Esbenshade, and B. H. Johnson. 1991. Cortisol, growth hormone, and testosterone concentrations during mating behavior in the bull and boar. J Anim Sci 69(8):3230-3240. doi: 10.2527/1991.6983230x \%J Journal of Animal Science

Brando, S., and H. M. Buchanan-Smith. 2018. The 24/7 approach to promoting optimal welfare for captive wild animals. Behavioural Processes 156:83-95. doi: https://doi.org/10.1016/j.beproc.2017.09.010

Bremner, W. J., M. V. Vitiello, and P. N. Prinz. 1983. Loss of Circadian Rhythmicity in Blood Testosterone Levels with Aging in Normal Men. The Journal of Clinical Endocrinology \& Metabolism 56(6):1278-1281. doi: 10.1210/jcem-56-6-1278 \%J The Journal of Clinical Endocrinology \& Metabolism 
Brown, J., P. Bansiddhi, J. Khonmee, and a. Thitaram. 2020. Commonalities in Management and Husbandry Factors Important for Health and Welfare of Captive Elephants in North America and Thailand. Animals 10:737. doi: 10.3390/ani10040737

Brown, J. L., K. Carlstead, J. D. Bray, D. Dickey, C. Farin, and K. Ange-van Heugten. 2019. Individual and environmental risk factors associated with fecal glucocorticoid metabolite concentrations in zoo-housed Asian and African elephants. PLoS One 14(9):e0217326. doi: https://doi.org/10.1371/journal.pone.0217326

Brown, J. L., M. Somerville, H. S. Riddle, M. Keele, C. K. Duer, and E. W. Freeman. 2007. Comparative endocrinology of testicular, adrenal and thyroid function in captive Asian and African elephant bulls. Gen Comp Endocrinol 151(2):153-162. doi: http://dx.doi.org/10.1016/j.ygcen.2007.01.006

Buchanan-Smith, H. M., and I. Badihi. 2012. The psychology of control: Effects of control over supplementary light on welfare of marmosets. Appl Anim Behav Sci 137(3):166-174. doi: 10.1016/j.applanim.2011.07.002

Cameron, E. Z., and S. J. Ryan. 2016. Welfare at Multiple Scales: Importance of Zoo Elephant Population Welfare in a World of Declining Wild Populations. PLoS One 11(7):e0158701. doi: 10.1371/journal.pone.0158701

Carlstead, K. 1996. Effects of captivity on the behavior of wild mammals. In: D. G. Kleiman, M. E. Allen, Thompson, and L. K.V., S, editors, Wild Mammals in Captivity: Principles and Techniques. University of Chicago Press, Chicago, USA. p. 317-333.

Chave, E., K. L. Edwards, S. Paris, N. Prado, K. A. Morfeld, and J. L. Brown. 2019. Variation in metabolic factors and gonadal, pituitary, thyroid, and adrenal hormones in association with musth in African and Asian elephant bulls. Gen Comp Endocrinol 276:1-13. doi: https://doi.org/10.1016/j.ygcen.2019.02.005

Cockrem, J. F. 2013. Individual variation in glucocorticoid stress responses in animals. Gen Comp Endocrinol 181:45-58. doi: https://doi.org/10.1016/j.ygcen.2012.11.025

Coleing, A. 2009. The application of social network theory to animal behaviour. Bioscience Horizons: The International Journal of Student Research 2(1):32-43. doi: 10.1093/biohorizons/hzp008 
de Silva, S., A. D. Ranjeewa, and S. Kryazhimskiy. 2011. The dynamics of social networks among female Asian elephants. BMC Ecology 11(1):17. (journal article) doi: $10.1186 / 1472-6785-11-17$

de Silva, S., and G. Wittemyer. 2012. A Comparison of Social Organization in Asian Elephants and African Savannah Elephants. Int J Primatol 33(5):1125-1141. doi: https://doi.org/10.1007/s10764-011-9564-1

DeVries, A. C., E. R. Glasper, and C. E. Detillion. 2003. Social modulation of stress responses. Physiol Behav 79(3):399-407. doi: https://doi.org/10.1016/S00319384(03)00152-5

Edwards, K. L., P. Bansiddhi, S. Paris, M. Galloway, and J. L. Brown. 2019. The development of an immunoassay to measure immunoglobulin A in Asian elephant feces, saliva, urine and serum as a potential biomarker of well-being. Conserv Physiol 7(1):coy077. doi: https://doi.org/10.1093/conphys/coy077

Ellis, S., D. W. Franks, S. Nattrass, M. A. Cant, D. L. Bradley, D. Giles, K. C. Balcomb, and D. P. Croft. 2018. Postreproductive lifespans are rare in mammals. Ecol Evol 8(5):2482-2494. doi: 10.1002/ece3.3856

Evans, K. E., and S. Harris. 2008. Adolescence in male African elephants, Loxodonta africana, and the importance of sociality. Anim Behav 76(3):779-787.

Fanson, K. V., T. Keeley, and B. G. Fanson. 2014. Cyclic changes in cortisol across the estrous cycle in parous and nulliparous Asian elephants. Endocr Connect 3(2):5766. doi: https://doi.org/10.1530/EC-14-0025

Garai, M., and F. Kurt. 2006. The importance of socialisation to the well being of elephants. Zeitschrift des Kölner Zoo 2(49):85-102.

Goymann, W., M. L. East, B. Wachter, O. P. Honer, E. Mostl, T. J. Van't Hof, and H. Hofer. 2001. Social, state-dependent and environmental modulation of faecal corticosteroid levels in free-ranging female spotted hyenas. Proc Biol Sci 268(1484):2453-2459. doi: 10.1098/rspb.2001.1828

Gunawardene, M., L. Jayasinghe, H. Janaka, D. Weerakoon, E. Wickramanayake, and P. Fernando. 2004. Social Organization of Elephants in southern Sri Lanka. In: Symposium for Human-Elephant Relationships and Conflicts, Colombo, Sri Lanka 
Hacker, C. E., K. M. Horback, and L. J. Miller. 2015. GPS technology as a proxy tool for determining relationships in social animals: An example with African elephants. Applied Animal Behaviour Science 163:175-182. doi: 10.1016/j.applanim.2014.12.005

Hartley, M., A. Wood, and L. Yon. 2019. Facilitating the social behaviour of bull elephants in zoos. 53(1):62-77. doi: 10.1111/izy.12245

Howland, B. E., L. M. Sanford, and W. M. Palmer. 1985. Changes in serum levels of LH, FSH, prolactin, testosterone, and cortisol associated with season and mating in male pygmy goats. J Androl 6(2):89-96. doi: 10.1002/j.19394640.1985.tb00822.x

Jayantha, D., P. Dayawansa, U. Padmalal, and W. J. J. o. T. T. Ratnasooriya. 2009. Social relationships of wild juvenile Asian Elephants Elephas maximus in the Udawalawa National Park, Sri Lanka. Journal of Threatened Taxa 1(4):211-214.

Kersey, D. C., D. E. Wildt, J. L. Brown, R. J. Snyder, Y. Huang, and S. L. Monfort. 2011. Rising fecal glucocorticoid concentrations track reproductive activity in the female giant panda (Ailuropoda melanoleuca). Gen Comp Endocrinol 173(2):364-370. doi: https://doi.org/10.1016/j.ygcen.2011.06.013

Khonmee, J., J. L. Brown, S. Rojanasthien, D. Thumasanukul, A. Kongphoemphun, B. Siriaroonrat, W. Tipkantha, V. Punyapornwithaya, and C. Thitaram. 2014. Seasonality of fecal androgen and glucocorticoid metabolite excretion in male goral (Naemorhedus griseus) in Thailand. Anim Reprod Sci 146(1):70-78. doi: https://doi.org/10.1016/j.anireprosci.2014.02.008

Koolhaas, J. M., S. M. Korte, S. F. De Boer, B. J. Van Der Vegt, C. G. Van Reenen, H. Hopster, I. C. De Jong, M. A. W. Ruis, and H. J. Blokhuis. 1999. Coping styles in animals: current status in behavior and stress-physiology. Neurosci Biobehav Rev 23(7):925-935. doi: https://doi.org/10.1016/S0149-7634(99)00026-3

Krebs, B. L., D. Marrin, A. Phelps, L. Krol, and J. V. Watters. 2018. Managing Aged Animals in Zoos to Promote Positive Welfare: A Review and Future Directions. Animals (Basel) 8(7):116. doi: 10.3390/ani8070116

Lee, B. 2019. AZA Regional Studbook for Asian Elephant (Elephas maximus). Association of Zoos and Aquariums, Portland, OR.

Lemaître, J.-F., and J.-M. Gaillard. 2017. Reproductive senescence: new perspectives in the wild. Biological Reviews 92(4):2182-2199. doi: 10.1111/brv.12328 
Liptrap, R., and J. Raeside. 1968. Effect of corticotrophin and corticosteroids on plasma interstitial cell-stimulating hormone and urinary steroids in the boar. J Endocrinol 42(1):33-43.

Liptrap, R. M., and J. I. Raeside. 1978. A relationship between plasma concentrations of testosterone and corticosteroids during sexual and aggressive behaviour in the boar. J Endocrinol 76(1):75-85. doi: 10.1677/joe.0.0760075

McKay, G. M. 1973. Behavior and ecology of the Asiatic elephant in southeastern Ceylon, Smithsonian Contributions to Zoology No. 125. Smithsonian Institution Press, Washington, DC. p. 1-113.

Meehan, C. L., and J. A. Mench. 2007. The challenge of challenge: Can problem solving opportunities enhance animal welfare? Appl Anim Behav Sci 102(3):246-261. doi: 10.1016/j.applanim.2006.05.031

Morley, J. E., F. E. Kaiser, H. M. Perry, P. Patrick, P. M. K. Morley, P. M. Stauber, B. Vellas, R. N. Baumgartner, and P. J. Garry. 1997. Longitudinal changes in testosterone, luteinizing hormone, and follicle-stimulating hormone in healthy older men. Metabolism 46(4):410-413. doi: https://doi.org/10.1016/S00260495(97)90057-3

Pesántez-Pacheco, J. L., A. Heras-Molina, L. Torres-Rovira, M. V. Sanz-Fernández, C. García-Contreras, M. Vázquez-Gómez, P. Feyjoo, E. Cáceres, M. Frías-Mateo, F. Hernández, P. Martínez-Ros, J. V. González-Martin, A. González-Bulnes, and S. Astiz. 2019. Maternal Metabolic Demands Caused by Pregnancy and Lactation: Association with Productivity and Offspring Phenotype in High-Yielding Dairy Ewes. Animals 9(6):295. doi: https://doi.org/10.3390/ani9060295

Pinto, A., M. Stelvig, C. Costa, J. Colaço, and B. Colaço. 2020. Influence of male presence on female Asian elephants (Elephas maximus) behaviour in captivity. Journal of Zoo and Aquarium Research 8(1):45-49.

Plangsangmas, T., J. L. Brown, C. Thitaram, A. Silva-Fletcher, K. L. Edwards, V. Punyapornwithaya, P. Towiboon, and C. Somgird. 2020. Circadian Rhythm of Salivary Immunoglobulin A and Associations with Cortisol as A Stress Biomarker in Captive Asian Elephants (Elephas maximus). Animals 10(1):157.

Pokharel, S. S., P. B. Seshagiri, and R. Sukumar. 2019. Influence of the number of calves and lactating adult females in a herd on the adrenocortical activity of free-ranging Asian elephants. Wildlife Research 46(8):679-689. doi: 10.1071/wr18163 
Prado-Oviedo, N. A., M. K. Bonaparte-Saller, E. J. Malloy, C. L. Meehan, J. A. Mench, K. Carlstead, and J. L. Brown. 2016. Evaluation of Demographics and Social Life Events of Asian (Elephas maximus) and African Elephants (Loxodonta africana) in North American Zoos. PLoS One 11(7):e0154750. doi:

https://doi.org/10.1371/journal.pone.0154750

Rault, J.-L. 2012. Friends with benefits: social support and its relevance for farm animal welfare. Appl Anim Behav Sci 136(1):1-14. doi: https://doi.org/10.1016/j.applanim.2011.10.002

Rees, P. A. 2004. Some Preliminary Evidence of the Social Facilitation of Mounting Behavior in a Juvenile Bull Asian Elephant (Elephas maximus). Journal of Applied Animal Welfare Science 7(1):49-58. doi: 10.1207/s15327604jaws0701_3

Ross, H. E., and L. J. Young. 2009. Oxytocin and the neural mechanisms regulating social cognition and affiliative behavior. Frontiers in Neuroendocrinology 30(4):534-547. doi: https://doi.org/10.1016/j.yfrne.2009.05.004

Samaras, N., D. Samaras, E. Frangos, A. Forster, and J. Philippe. 2013. A review of agerelated dehydroepiandrosterone decline and its association with well-known geriatric syndromes: is treatment beneficial? Rejuvenation research 16(4):285294.

Schiml, P. A., and E. F. Rissman. 1999. Cortisol facilitates induction of sexual behavior in the female musk shrew (Suncus murinus). Behav Neurosci 113(1):166-175. doi: https://doi.org/10.1037/0735-7044.113.1.166

Sosa, C., F. Forcada, A. Meikle, and J. A. Abecia. 2013. Increase in ovine plasma cortisol at oestrus and its relation with the metabolic status during the sexual cycle in sheep. Biol Rhythm Res 44(3):445-449. doi: https://doi.org/10.1080/09291016.2012.704793

Spinage, C. 1994. Elephants. . T \& AD Poyser Ltd., London.

Staley, M., M. G. Conners, K. Hall, and L. J. Miller. 2018. Linking stress and immunity: Immunoglobulin A as a non-invasive physiological biomarker in animal welfare studies. Horm Behav 102:55-68. doi: https://doi.org/10.1016/j.yhbeh.2018.04.011

Sukumar, R. 2003. The living elephants : evolutionary ecology, behavior, and conservation. Oxford University Press, New York. 
Thongtip, N., J. Saikhun, S. Mahasawangkul, K. Kornkaewrat, P. Pongsopavijitr, N. Songsasen, and A. Pinyopummin. 2008. Potential factors affecting semen quality in the Asian elephant (Elephas maximus). Reproductive Biology and Endocrinology 6(1):9. doi: 10.1186/1477-7827-6-9

Veasey, J. S. 2020. Assessing the Psychological Priorities for Optimising Captive Asian Elephant (Elephas maximus) Welfare. Animals 10(1):39. doi: 10.3390/ani10010039

Wang, C., A. Leung, and A. P. Sinha-Hikim. 1993. Reproductive aging in the male brown-Norway rat: a model for the human. Endocrinology 133(6):2773-2781. doi: 10.1210/endo.133.6.8243304\%J Endocrinology

Watters, J., K. Sulzner, D. Marrin, S. Huang, C. MacDonald, S. Ostapak, A. Poole, and H. Hayle. 2015. Assessing quality of life in geriatric zoo animals. World Association of Zoos and Aquariums 16:37.

Yon, L., S. Kanchanapangka, N. Chaiyabutr, S. Meepan, F. Z. Stanczyk, N. Dahl, and B. Lasley. 2007. A longitudinal study of LH, gonadal and adrenal steroids in four intact Asian bull elephants (Elephas maximus) and one castrate African bull (Loxodonta africana) during musth and non-musth periods. General and Comparative Endocrinology 151(3):241-245. doi: https://doi.org/10.1016/j.ygcen.2007.01.028

Yon, L., E. Williams, N. D. Harvey, and L. Asher. 2019. Development of a behavioural welfare assessment tool for routine use with captive elephants. PLoS One 14(2):e0210783. doi: 10.1371/journal.pone.0210783 
Appendix A: Longitudinal Hormone Concentrations (Chapter 2)

\begin{tabular}{|c|c|c|c|c|c|c|c|c|c|}
\hline \multirow{4}{*}{ 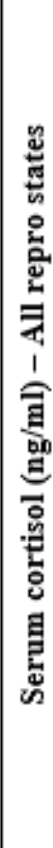 } & : & 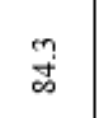 & 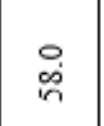 & $\frac{9}{\infty}$ & gे & $\begin{array}{l}\infty \\
\stackrel{0}{+} \\
\stackrel{0}{+}\end{array}$ & $\stackrel{\infty}{\stackrel{\infty}{n}}$ & $\stackrel{\infty}{\vec{b}}$ & $\stackrel{\circ}{\stackrel{+}{2}}$ \\
\hline & 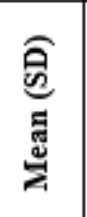 & 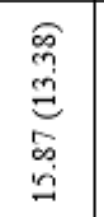 & 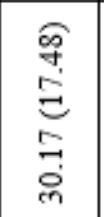 & 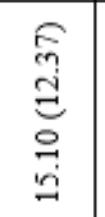 & 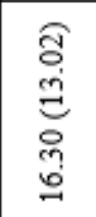 & 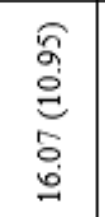 & 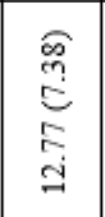 & 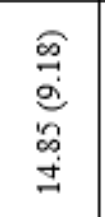 & 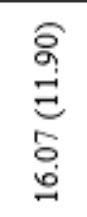 \\
\hline & 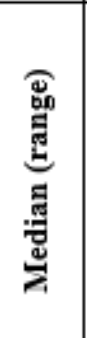 & 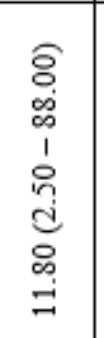 & 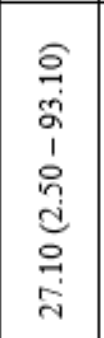 & 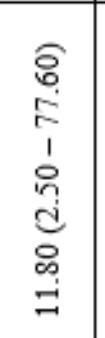 & 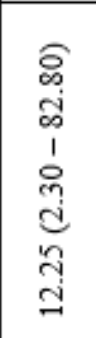 & 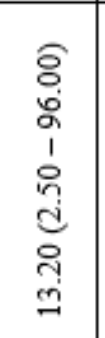 & 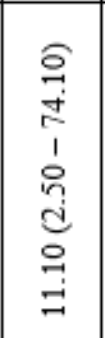 & 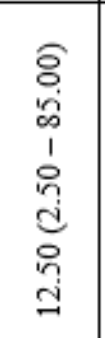 & 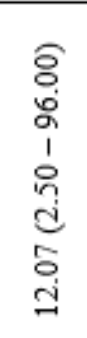 \\
\hline & $\mathrm{z}$ & $\overbrace{n}^{\infty}$ & নి & 兽 & $\begin{array}{l}\stackrel{\infty}{\circ} \\
\stackrel{\infty}{q}\end{array}$ & 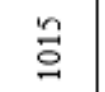 & 怘 & 号 & 怘 \\
\hline & $\begin{array}{l}\text { 至 } \\
\text { 至 }\end{array}$ & 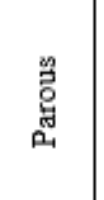 & 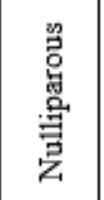 & 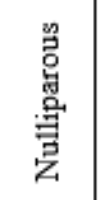 & 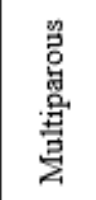 & 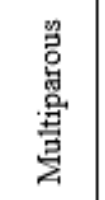 & 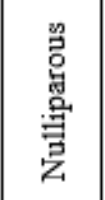 & 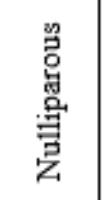 & \\
\hline & 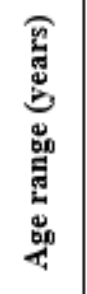 & $\vec{J}$ & $\frac{2}{1}$ & 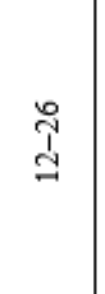 & $\begin{array}{l}\overrightarrow{\hat{1}} \\
\text { ô }\end{array}$ & $\begin{array}{l}9 \\
? \\
\frac{9}{2}\end{array}$ & 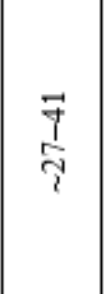 & $\begin{array}{l}\vec{T} \\
\vdots \\
i\end{array}$ & $\begin{array}{l}\vec{F} \\
\dot{2} \\
\stackrel{9}{+}\end{array}$ \\
\hline & 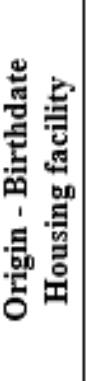 & 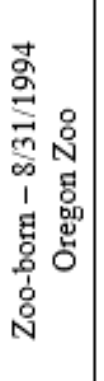 & 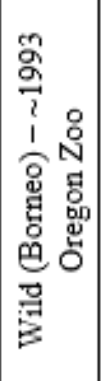 & 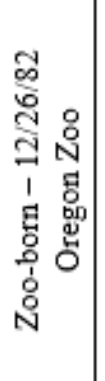 & 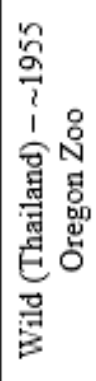 & 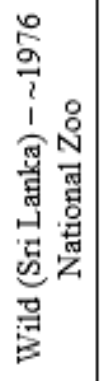 & 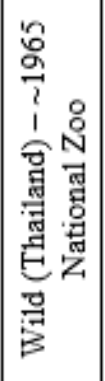 & 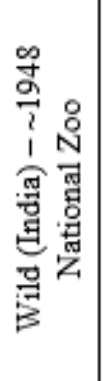 & \\
\hline & 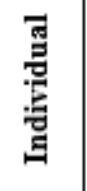 & $\stackrel{N}{\stackrel{N}{\Psi}}$ & 㺼 & 孞 & $\begin{array}{l}\text { N } \\
\text { O } \\
\text { 岃 }\end{array}$ & 忩 & $\begin{array}{c}N \\
\text { N } \\
\text { 出 }\end{array}$ & $\underset{⿱ 乛 龰}{\stackrel{N}{N}}$ & 表㺃产 \\
\hline
\end{tabular}




\begin{tabular}{|c|c|c|c|c|c|c|c|c|}
\hline & :̊ & 袁 & 웅 & $\overrightarrow{\mathrm{\sigma}}$ & $\begin{array}{l}\infty \\
\dot{f} \\
\end{array}$ & $\stackrel{\circ}{i}$ & $\frac{2}{a}$ & 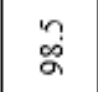 \\
\hline 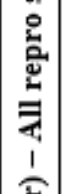 & 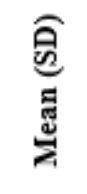 & 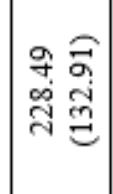 & 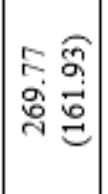 & 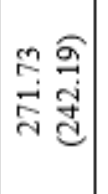 & 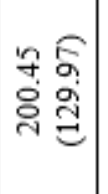 & 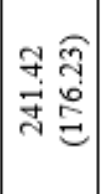 & 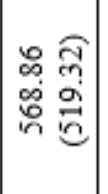 & 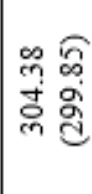 \\
\hline 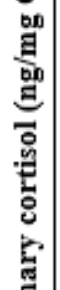 & 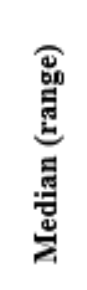 & 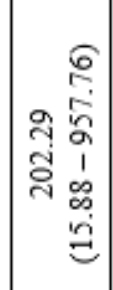 & 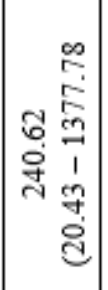 & 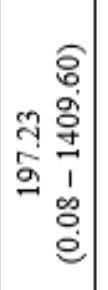 & 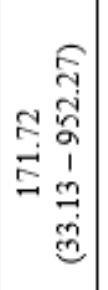 & 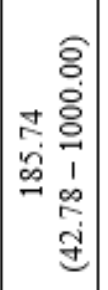 & 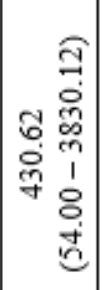 & 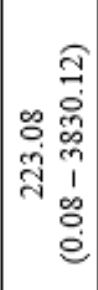 \\
\hline & $z$ & ते & 气े & ๕ & $\Xi$ & $m_{\pi}$ & I & 总 \\
\hline & 至 & 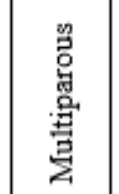 & 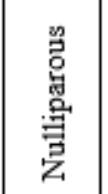 & 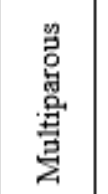 & 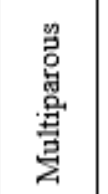 & 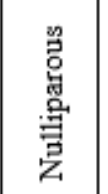 & 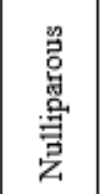 & \\
\hline & 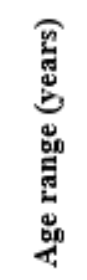 & 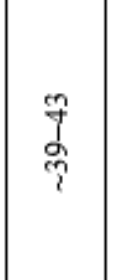 & $\begin{array}{l}\overrightarrow{1} \\
\vdots \\
i\end{array}$ & $\underset{\mathbb{T}}{\mathbb{T}}$ & $\begin{array}{l}\text { 等 } \\
\text { के }\end{array}$ & 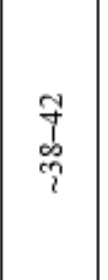 & 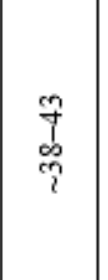 & \begin{tabular}{|l|}
$\vec{r}$ \\
$\dot{z}$ \\
$\dot{d}$ \\
$\vec{d}$
\end{tabular} \\
\hline & 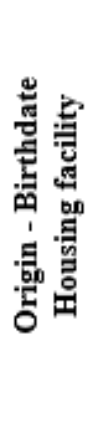 & 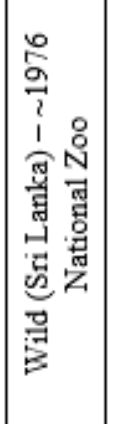 & 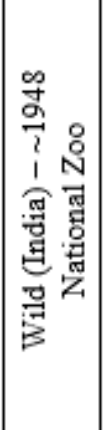 & 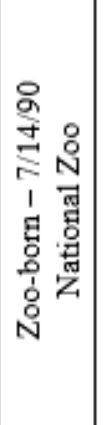 & 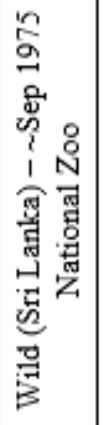 & 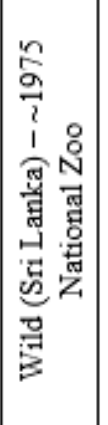 & 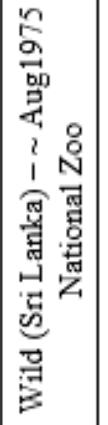 & \\
\hline & 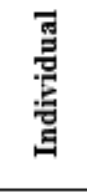 & 营 & 窋 & 总 & 营 & 营 & 萤 & 表量 \\
\hline
\end{tabular}




\begin{tabular}{|c|c|c|c|c|c|c|c|c|c|}
\hline 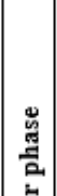 & 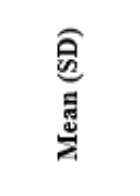 & 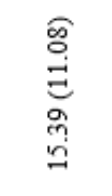 & 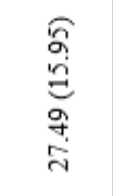 & 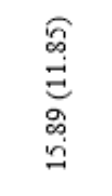 & 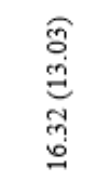 & 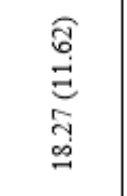 & 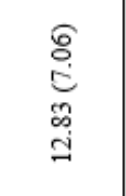 & 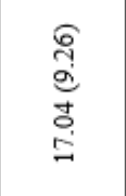 & 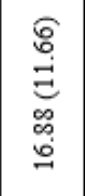 \\
\hline 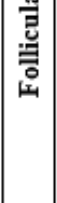 & 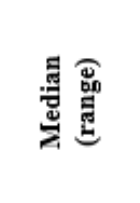 & 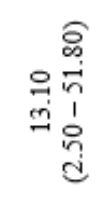 & 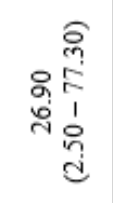 & 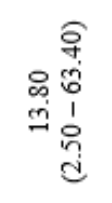 & 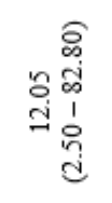 & 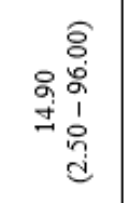 & 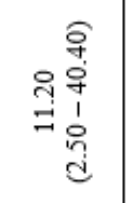 & 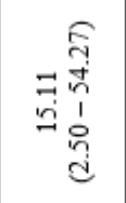 & $\begin{array}{l}P \\
\qquad \\
g\end{array}$ \\
\hline 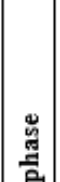 & 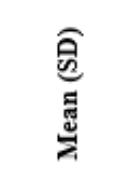 & 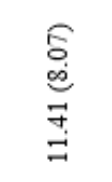 & 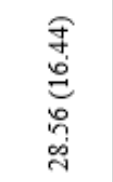 & 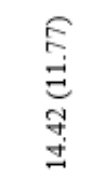 & $\begin{array}{l}\widehat{\vec{b}} \\
\text { త్ } \\
\text { oे } \\
\text { है } \\
0\end{array}$ & 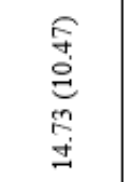 & 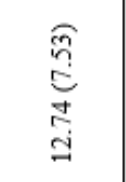 & $\begin{array}{l}\hat{6} \\
\stackrel{5}{5} \\
5 \\
\dot{y}\end{array}$ & 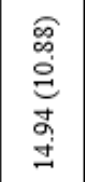 \\
\hline 學 & $\begin{array}{l}\text { 䔅 } \\
\text { 总 } \\
\text { 昜 }\end{array}$ & 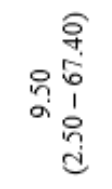 & 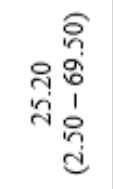 & 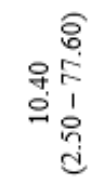 & 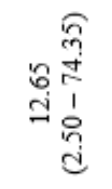 & 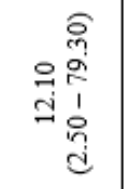 & 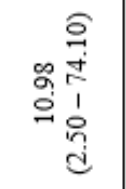 & 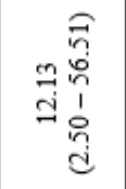 & $\stackrel{\text { 岕 }}{=}$ \\
\hline 坣 & 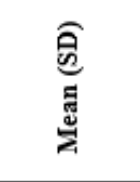 & $\begin{array}{l}\stackrel{2}{a} \\
a \\
a \\
a \\
a\end{array}$ & 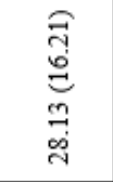 & 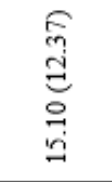 & 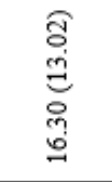 & 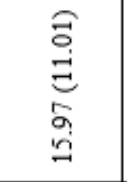 & 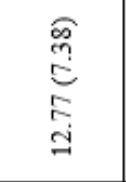 & 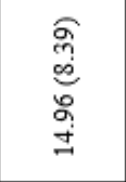 & 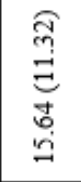 \\
\hline 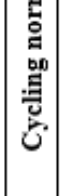 & 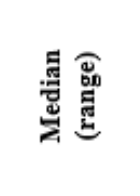 & 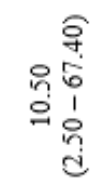 & 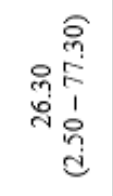 & 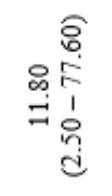 & 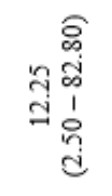 & 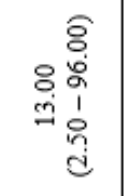 & 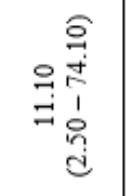 & 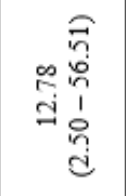 & 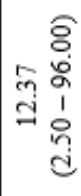 \\
\hline & z & $\stackrel{\vec{\infty}}{\sim}$ & 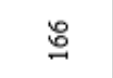 & $\stackrel{9}{\stackrel{9}{n}}$ & $\stackrel{\text { \& }}{\stackrel{一}{+}}$ & $\overrightarrow{\mathbb{N}}$ & $\stackrel{\circ}{\curvearrowleft}$ & 음 & : \\
\hline & 莺 & 䍘 & $\begin{array}{l}\text { 号 } \\
\text { 总 } \\
\text { 言 } \\
\text { 号 }\end{array}$ & 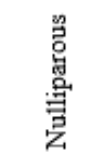 & 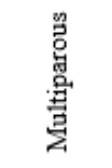 & 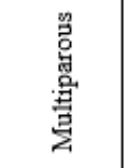 & 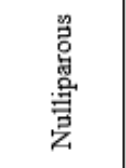 & 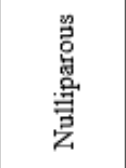 & \\
\hline & 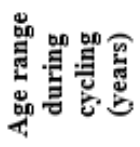 & $\stackrel{J}{I}$ & $\begin{array}{l}\frac{9}{1} \\
\frac{1}{2}\end{array}$ & $\begin{array}{l}\stackrel{0}{1} \\
\text { ป }\end{array}$ & î̀ & $\begin{array}{l}\frac{m}{7} \\
\frac{2}{2}\end{array}$ & $\frac{\vec{I}}{\stackrel{7}{7}}$ & $\begin{array}{l}\text { î̀ } \\
\text { iे }\end{array}$ & $\begin{array}{l}\text { aे } \\
? \\
q \\
+ \\
+\end{array}$ \\
\hline & 焉密 & 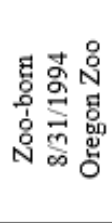 & 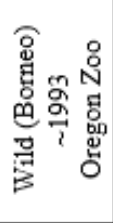 & 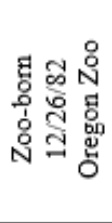 & 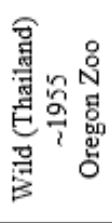 & 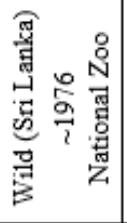 & 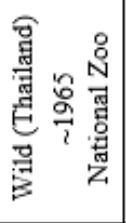 & 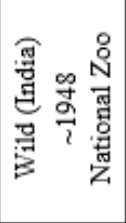 & \\
\hline & 䨚 & 奋 & $\begin{array}{l}\mathrm{N} \\
\text { 어 }\end{array}$ & 息 & $\begin{array}{l}N \\
\text { O } \\
\text { 虫 }\end{array}$ & 旁 & $\begin{array}{l}N \\
\text { N } \\
\text { 至 }\end{array}$ & $\underset{⿱ 亡}{\mathrm{~N}}$ & 表营 \\
\hline
\end{tabular}




\begin{tabular}{|c|c|c|c|c|c|}
\hline \multirow{2}{*}{ 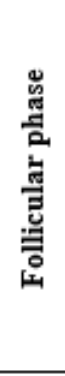 } & 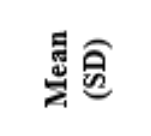 & 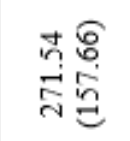 & 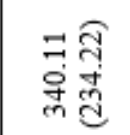 & 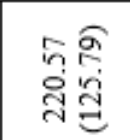 & 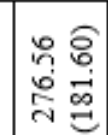 \\
\hline & 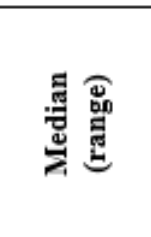 & 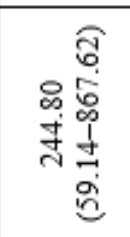 & 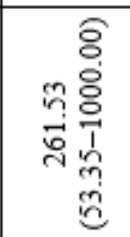 & 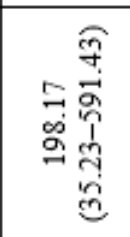 & 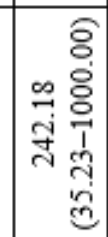 \\
\hline \multirow{2}{*}{ 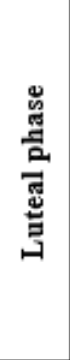 } & 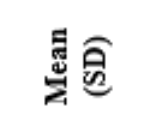 & 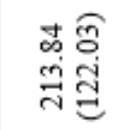 & 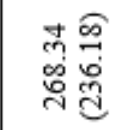 & 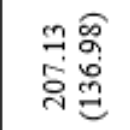 & 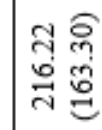 \\
\hline & 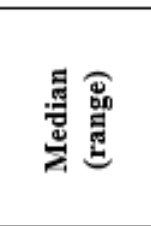 & 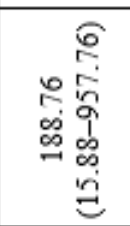 & 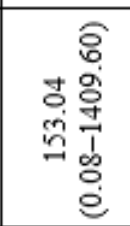 & 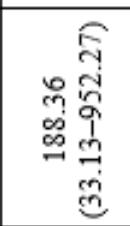 & 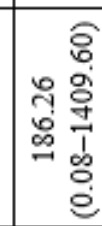 \\
\hline \multirow{3}{*}{ 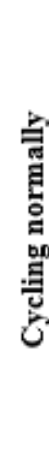 } & 善 商 & 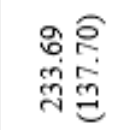 & 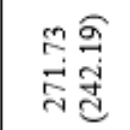 & 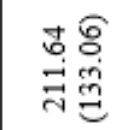 & 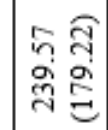 \\
\hline & 憘品 & 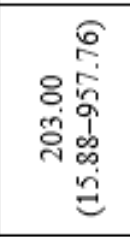 & 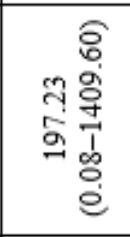 & 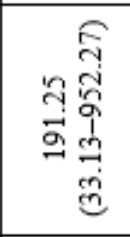 & 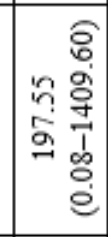 \\
\hline & $z$ & $\stackrel{\circ}{.}$ & $\stackrel{\circ}{\circ}$ & $\Xi$ & 吉 \\
\hline & 点 & 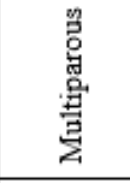 & 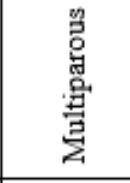 & 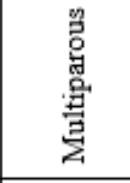 & \\
\hline & 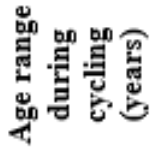 & $\begin{array}{l}\text { के } \\
\text { के } \\
\text { ? }\end{array}$ & 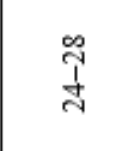 & $\begin{array}{l}\text { ô } \\
\text { के } \\
\text { ? }\end{array}$ & 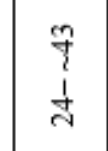 \\
\hline & 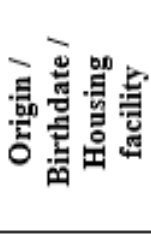 & 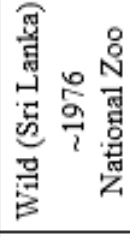 & 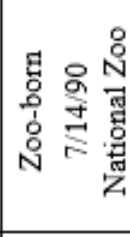 & 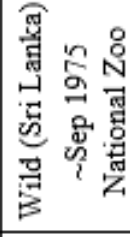 & \\
\hline & $\begin{array}{l}\text { 哥 } \\
\text { 量 }\end{array}$ & 营 & 窝 & 窝 & 寻售 \\
\hline
\end{tabular}


S2 Table A: Coefficient of variation $(\mathrm{CV})$ in cortisol concentration for reproductive states. Individual, CV percentage for reproductive state, Brown-Forsythe Test statistic, and p-value for comparisons.

\begin{tabular}{|c|c|c|c|c|c|c|c|}
\hline \multirow{2}{*}{ Individual } & \multicolumn{6}{|c|}{ CV $\%$} & \multirow[t]{2}{*}{ BF Test } \\
\hline & Prepubertal & Cycling & Pregnant & $\begin{array}{l}\text { Lactation } \\
\text { Anestrous }\end{array}$ & $\begin{array}{c}\text { Contracepted } \\
\text { / Acyclic }\end{array}$ & $\begin{array}{c}\text { Irregular } \\
\text { Cycling }\end{array}$ & \\
\hline \multirow[t]{7}{*}{$\mathrm{F} 1 \mathrm{OZ}$} & 56.2 & 74.2 & 81.2 & 87.8 & & & $F^{*}(3,115)=22.83, \mathbf{p}=<0.001$ \\
\hline & $\mathrm{x}$ & $\mathrm{x}$ & & & & & $F^{*}(1,99.0)=12.15, \mathbf{p}=<\mathbf{0 . 0 0 1}$ \\
\hline & $\mathrm{x}$ & & $\mathrm{x}$ & & & & $F^{*}(1,95.0)=20.63, \mathbf{p}=<\mathbf{0 . 0 0 1}$ \\
\hline & $\mathrm{x}$ & & & $\mathrm{x}$ & & & $F^{*}(1,51.9)=50.68, \mathbf{p}=<0.001$ \\
\hline & & $\mathrm{x}$ & $\mathrm{x}$ & & & & $F^{*}(1,92.8)=6.95, \mathbf{p}=\mathbf{0 . 0 1 0}$ \\
\hline & & $\mathrm{x}$ & & $\mathrm{x}$ & & & $F^{*}(1,50.0)=34.37, \mathbf{p}=<\mathbf{0 . 0 0 1}$ \\
\hline & & & $\mathrm{x}$ & $\mathrm{x}$ & & & $F^{*}(1,78.3)=12.92, \mathbf{p}=<\mathbf{0 . 0 0 1}$ \\
\hline $\mathrm{F} 2 \mathrm{OZ}$ & 55.2 & 57.6 & & & & & $F^{*}(1,95.9)=7.17, \mathbf{p}=0.009$ \\
\hline \multirow{7}{*}{$\begin{array}{c}\text { F5NZ } \\
\text { (serum) }\end{array}$} & & 70.0 & 55.0 & 46.8 & 79.5 & & $F^{*}(3,187)=3.04, \mathbf{p}=<0.030$ \\
\hline & & $\mathrm{x}$ & $\mathrm{x}$ & & & & $F^{*}(1,251)=1.69, \mathrm{p}=0.194$ \\
\hline & & $\mathrm{x}$ & & $\mathrm{x}$ & & & $F^{*}(1,38.3)=7.53, \mathbf{p}=0.009$ \\
\hline & & $\mathrm{x}$ & & & $\mathrm{x}$ & & $F^{*}(1,80.3)=0.362, \mathrm{p}=0.549$ \\
\hline & & & $\mathrm{x}$ & $\mathrm{x}$ & & & $F^{*}(1,46.9)=10.19, \mathbf{p}=\mathbf{0 . 0 0 3}$ \\
\hline & & & $\mathrm{x}$ & & $\mathrm{x}$ & & $F^{*}(1,102)=0.008, \mathrm{p}=0.929$ \\
\hline & & & & $\mathrm{x}$ & $\mathrm{x}$ & & $F^{*}(1,82.7 \mathrm{x})=6.42, \mathbf{p}=\mathbf{0 . 0 1 3}$ \\
\hline $\begin{array}{l}\text { F5NZ } \\
\text { (urine) }\end{array}$ & & 59.2 & & & 44.9 & & $F^{*}(1,48.6)=2.79, \mathrm{p}=0.102$ \\
\hline F7NZ & & 55.8 & & & 70.5 & & $F^{*}(1,574)=0.045, \mathrm{p}=0.832$ \\
\hline F9NZ & & 62.9 & & & & 55.5 & $F^{*}(1,48.4)=23.8, \mathbf{p}=<\mathbf{0 . 0 0 1}$ \\
\hline
\end{tabular}

$\mathrm{X}$ denotes pair-wise comparisons made in the Brown-Forsythe statistic 
S2 Table B: Coefficient of variation (CV) in cortisol concentration for ovarian cyclicity. Individual, sample, CVs for ovarian cycle phases, Brown-Forsythe Test statistic, and p-value.

\begin{tabular}{|c|c|c|c|l|}
\hline \multirow{2}{*}{ Individual } & \multirow{2}{*}{ Sample } & \multicolumn{2}{|c|}{ CV\% } & \multirow{2}{*}{ BF Test } \\
\cline { 3 - 4 } & & Follicular & Luteal & \\
\hline All elephants & Serum & 69.1 & 72.8 & $F^{*}(1,1963)=19.42, \mathbf{p}=<\mathbf{0 . 0 0 1}$ \\
\hline All elephants (all parous) & Urine & 65.7 & 75.5 & $F^{*}(1,303)=12.82, \mathbf{p}=<\mathbf{0 . 0 0 1}$ \\
\hline Parous females & Serum & 69.8 & 74.5 & $F^{*}(1,913)=11.45, \mathbf{p}=<\mathbf{0 . 0 0 1}$ \\
\hline Nulliparous females & Serum & 68.3 & 71.4 & $F^{*}(1,1046)=8.12, \mathbf{p}=\mathbf{0 . 0 0 4}$ \\
\hline F1OZ & Serum & 72.0 & 70.7 & $F^{*}(1,142)=7.31, \mathbf{p}=\mathbf{0 . 0 0 8}$ \\
\hline F5NZ & Serum & 63.6 & 71.1 & $F^{*}(1,470)=16.28, \mathbf{p}=<\mathbf{0 . 0 0 1}$ \\
\hline F5NZ & Urine & 58.1 & 57.1 & $F^{*}(1,104)=6.52, \mathbf{p}=\mathbf{0 . 0 1 2}$ \\
\hline F7NZ & Serum & 54.3 & 53.8 & $F^{*}(1,241)=11.65, \mathbf{p}=\mathbf{0 . 0 0 7}$ \\
\hline F8NZ & Urine & 68.9 & 88.0 & $F^{*}(1,96.8)=7.53, \mathbf{p}=\mathbf{0 . 0 0 7}$ \\
\hline F3OZ & Serum & 74.6 & 89.4 & $F^{*}(1,324)=1.18, \mathrm{p}=0.278$ \\
\hline F2OZ & Serum & 58.0 & 57.6 & $F^{*}(1,145)=0.174, \mathrm{p}=0.676$ \\
\hline F4OZ & Serum & 79.8 & 76.9 & $F^{*}(1,268)=0.003, \mathrm{p}=0.954$ \\
\hline F6NZ & Serum & 55.0 & 59.1 & $F^{*}(1,355)=0.209, \mathrm{p}=0.648$ \\
\hline F9NZ & Urine & 57.0 & 66.1 & $F^{*}(1,108)=0.364, \mathrm{p}=0.547$ \\
\hline
\end{tabular}


Appendix B: Charted Predictions for Pairwise Comparisons (Chapter 2)

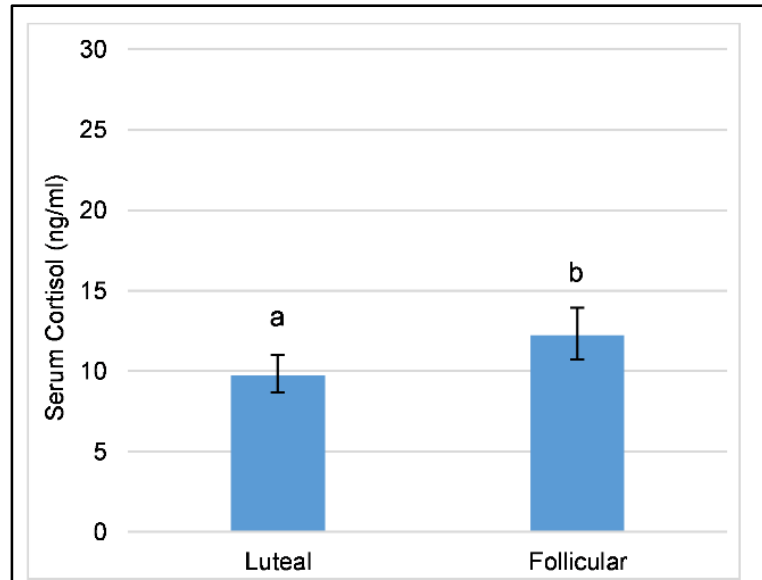

(A)
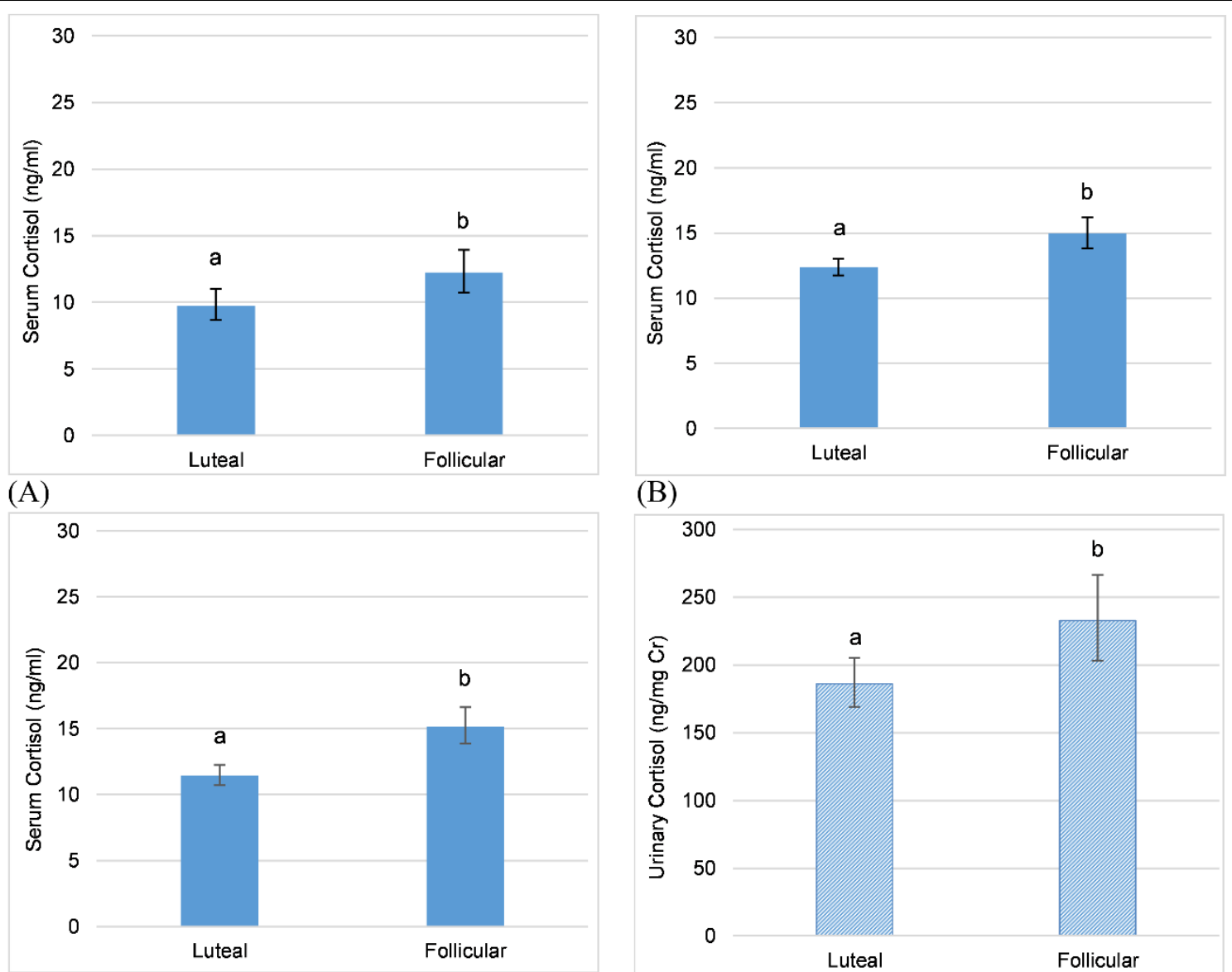

(B)

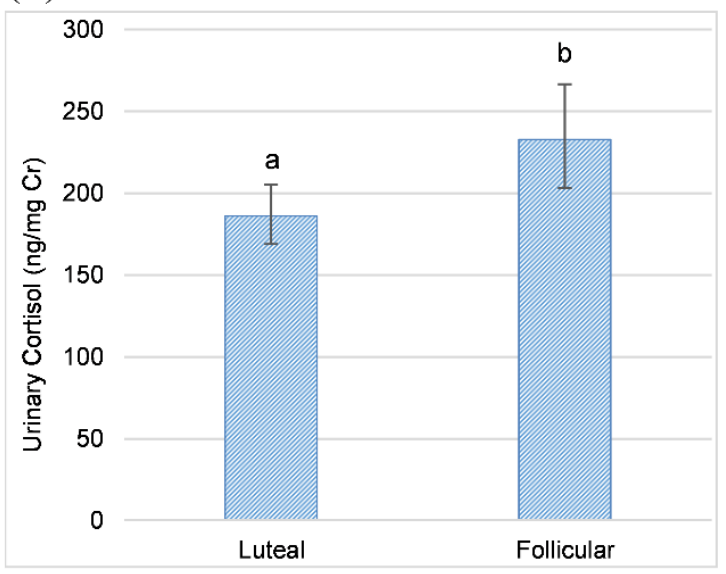

(C)

(D)

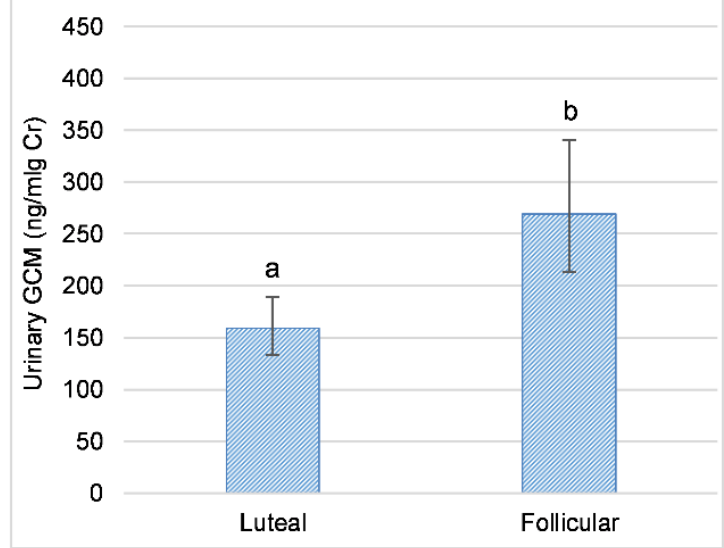

(E)

S3 Fig A: Ovarian cycle phase as a predictor of adrenal GC activity in individuals. Predictions from GLMMs for mean cortisol concentrations in the luteal phase and follicular phase of the ovarian cycle for individuals (error bars represent standard error of the prediction). Letters denote significant differences in hormone concentration between cycle phases. (A) F1OZ. (B) F7NZ. (C) F5NZ: serum cortisol. (D) F5NZ: urinary cortisol. (E) F8NZ. 

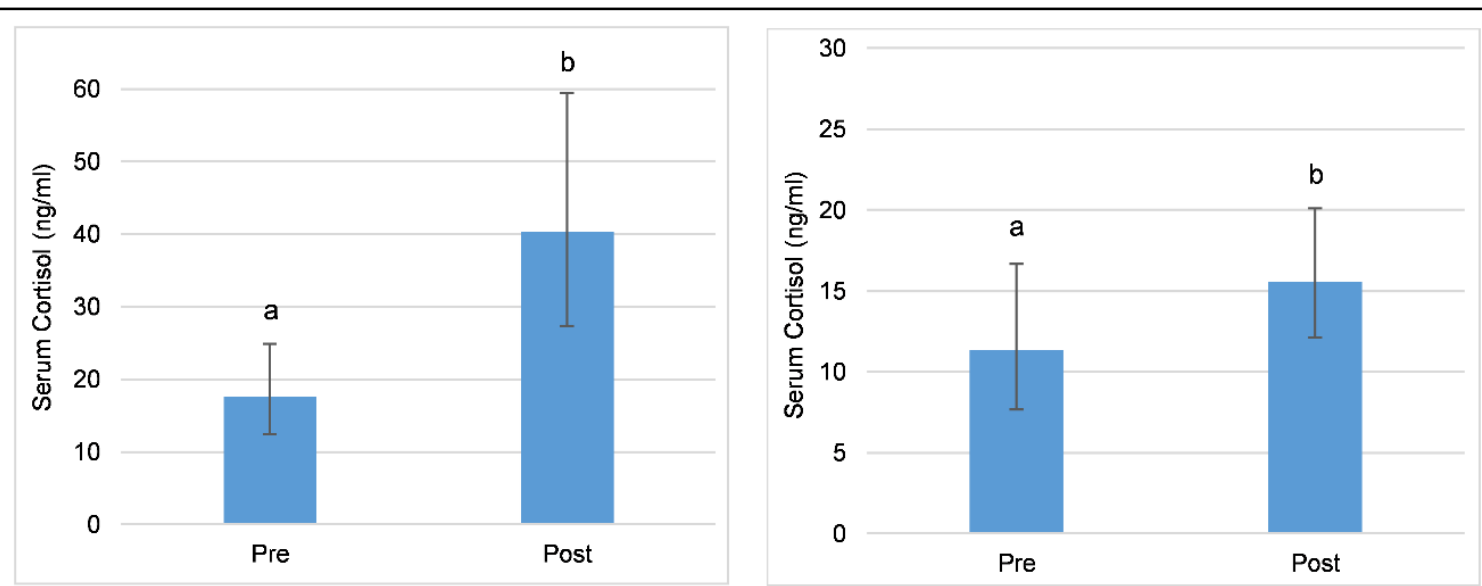

(A)

(B)

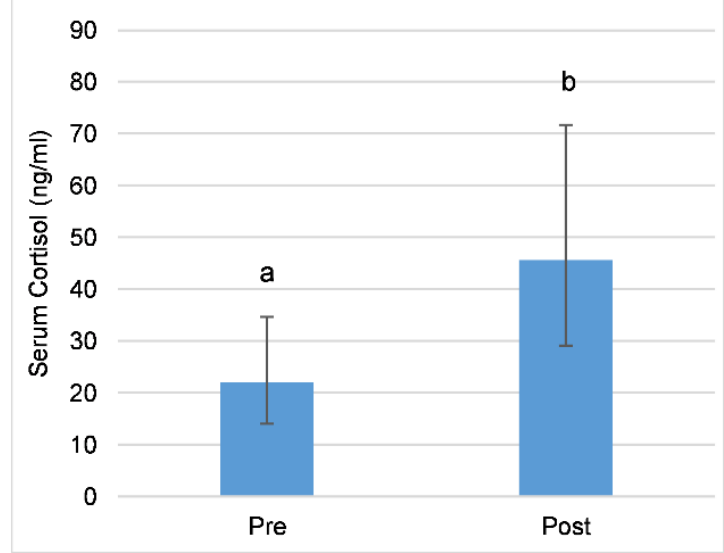

(C)

S3 Fig B: Death of a herdmate as a predictor of adrenal GC activity. Predictions from GLMMs for mean cortisol concentration in the 30 days prior to death and the 30 days post-death (error bars represent standard error of the prediction). Letters denote a significant difference in hormone concentration between pre- and post-death. (A) Death 1, F2OZ response: post > pre, comparing 30 days pre-post death. (B) Death 1, F3OZ response: post > pre, comparing 30 days pre-post death. (C) Death 4, F4OZ response: post > pre, comparing 30 days pre-post death. 


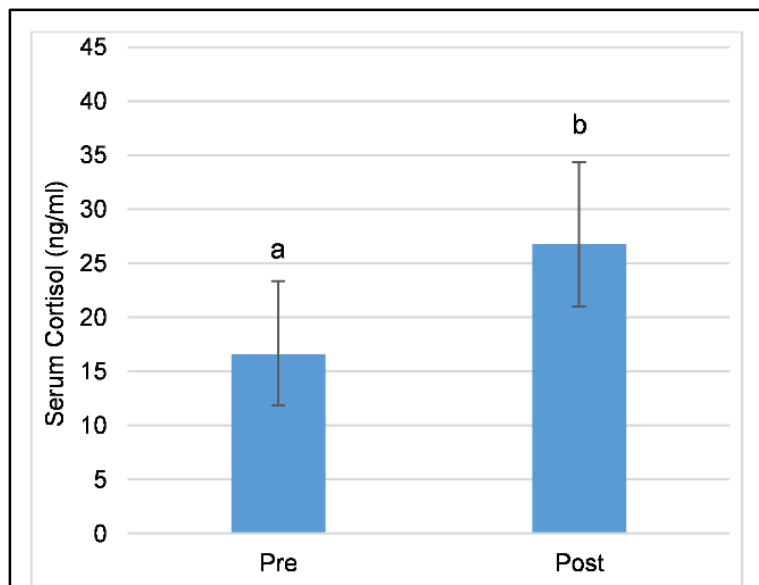

(A)

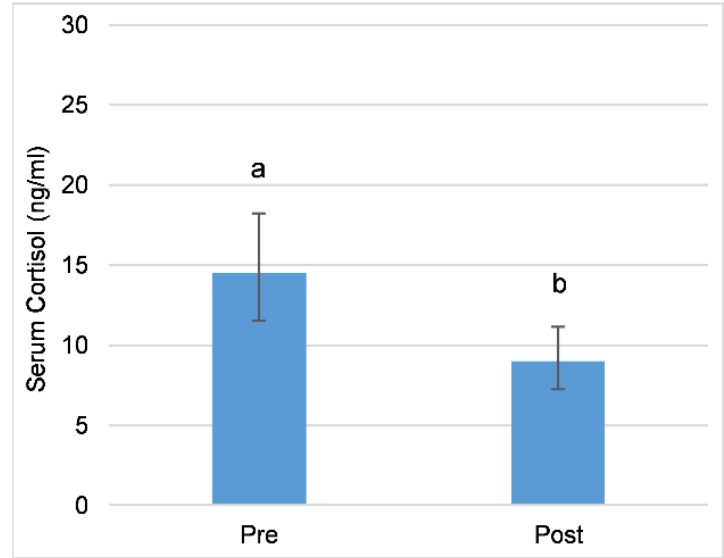

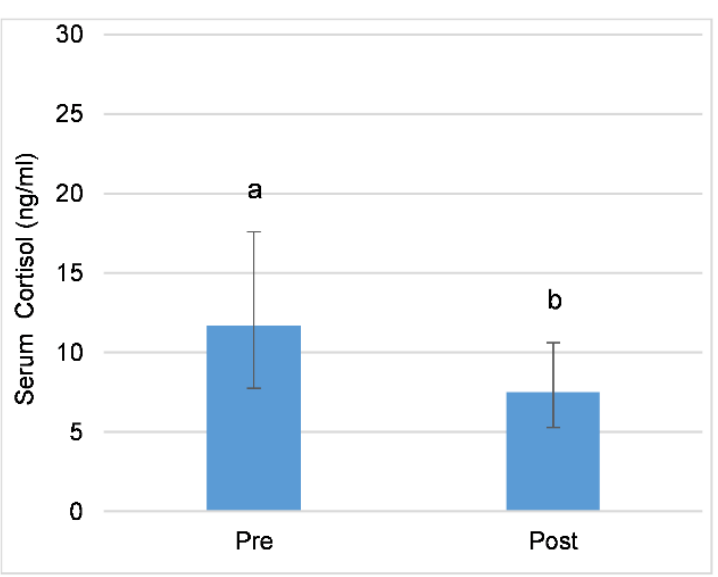

(B)

(C)

S3 Fig C: Transfer in of a herdmate a predictor of adrenal GC activity. Predictions from GLMMs for mean cortisol concentration in the 120 days prior to transfer and 120 days post-transfer in (error bars represent standard error of the prediction). Letters denote a significant difference in hormone concentration between pre- and post-transfer. (A) Transfer 2, F2OZ response: post > pre, comparing 120 days pre-post transfer. (B) Transfer 2, F3OZ response: post < pre, comparing 120 days pre-post transfer. (C) Transfer 2, F4OZ response: post < pre, comparing 120 days pre-post transfer. 


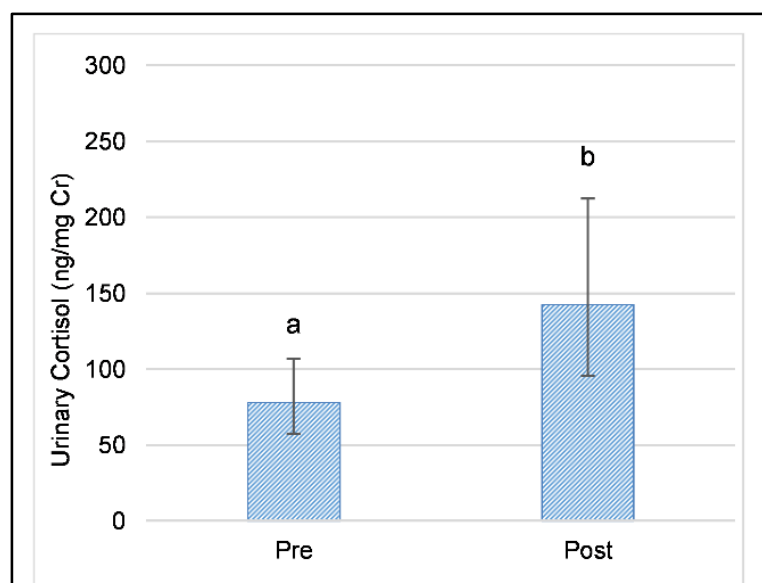

(A)

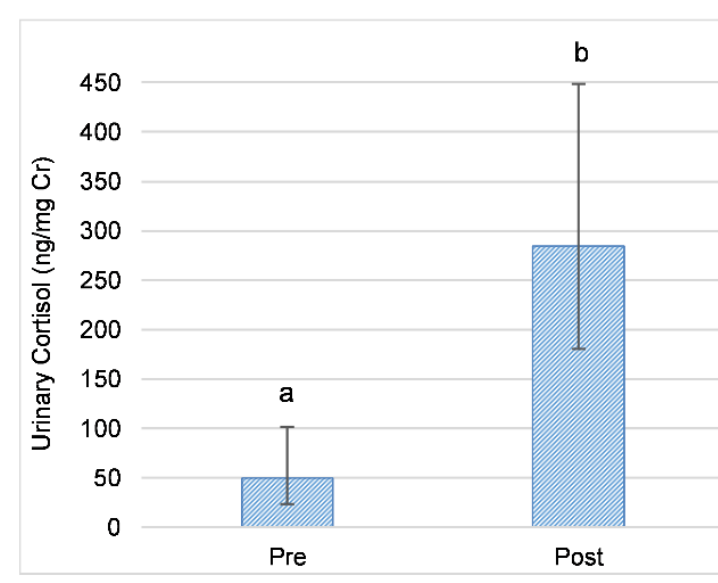

(B)

S3 Fig D: Transfer out of a herdmate as a predictor of adrenal GC activity. Predictions from GLMMs for mean cortisol concentrations in the 30 days prior to transfer and 30 days post-transfer (error bars represent standard error of the prediction). Letters denote a significant difference in hormone concentration between pre- and post-transfer. (A) Transfer 6, F5NZ response: post > pre, comparing 30 days pre-post transfer. (B) Transfer 6, F9NZ response: post > pre, comparing 30 days pre-post transfer.

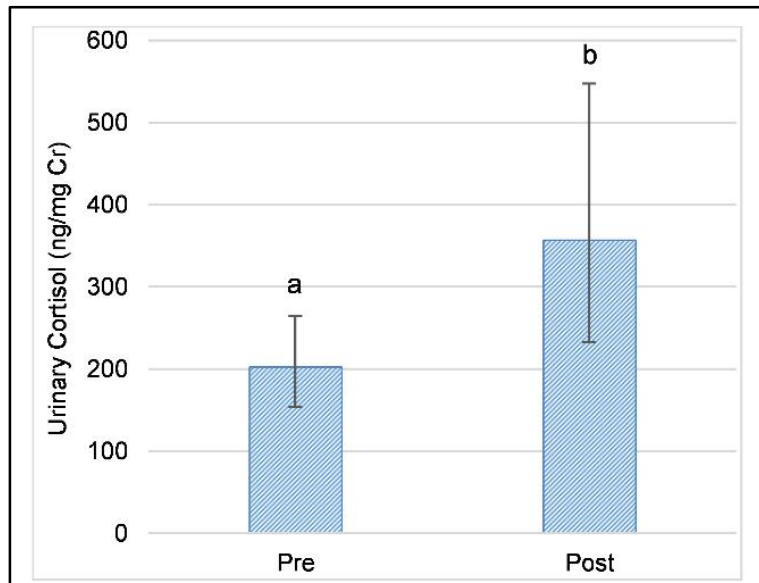

(A)

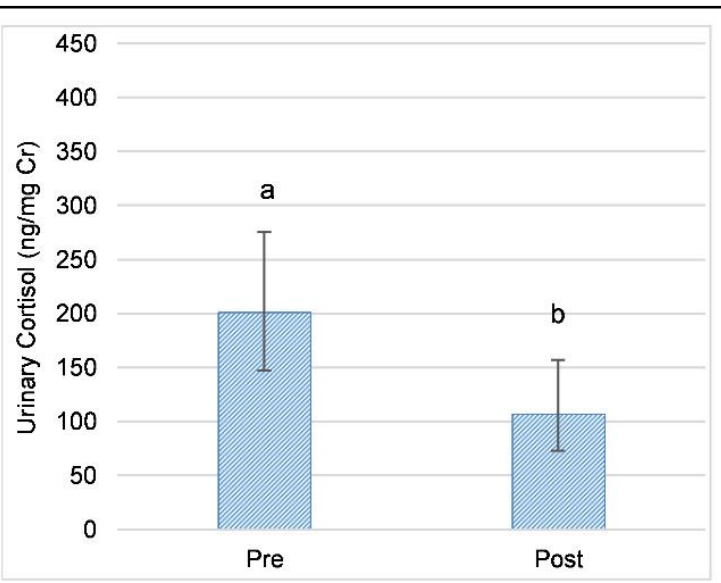

(B)

S3 Fig E: Transfer in of self as a predictor of adrenal GC activity. Predictions from GLMMs for mean cortisol concentrations in the 30 days prior to transfer and 30 days post-transfer (error bars represent standard error of the prediction). Letters denote a significant difference in hormone concentration between pre- and post-transfer. (A) Transfer of self, F8NZ: post > pre, comparing 30 days pre-post transfer. (B) Transfer of self, F9NZ: post > pre, comparing 30 days pre-post transfer. 
Appendix C: Elephant Behavior Ethogram (full version) (Chapter 4)

\section{Level 1: Behavior categories}

\begin{tabular}{|l|l|}
\hline \multicolumn{1}{|c|}{ Proximity } & \multicolumn{1}{c|}{ Definition } \\
\hline Proximate & $\begin{array}{l}\text { Focal is within 2 body length of or in contact with another individual. } \\
\text { 2 body lengths defined as: 2 adult female body lengths (c.a. } 10 \mathrm{~m} \text { ) }\end{array}$ \\
\hline $\begin{array}{l}\text { Proximity Not } \\
\text { Visible }\end{array}$ & $\begin{array}{l}\text { The focal animal itself or other elephants are not visible enough to } \\
\text { determine proximity. }\end{array}$ \\
\hline
\end{tabular}

\begin{tabular}{|c|c|}
\hline Behavior Category & Definition \\
\hline Behavior Not Visible & Elephant or activity is not visible enough to determine the behavior. \\
\hline Social Interaction & $\begin{array}{l}\text { Interacting with another elephant in a social context, either with } \\
\text { physical contact (e.g., trunk twine, play) or without physical contact } \\
\text { but within } 2 \text { body lengths (e.g., sharing food, displace). } \\
\text { Level2: Social behavior }\end{array}$ \\
\hline $\begin{array}{l}\text { Food Object } \\
\text { Interaction }\end{array}$ & $\begin{array}{l}\text { Interacting with any item that distributes food, either permanent or } \\
\text { keeper-provided. } \\
\text { Modifier: Food object } \\
\text { Food Object v. Enrichment objects: For items that can be either (e.g., } \\
\text { barrel), determined by whether the elephant appears to be eating } \\
\text { before/after interacting with the object. } \\
\text { Food Object v. Feeding: Determined by whether the elephant must } \\
\text { manipulate the object to get food (e.g., hay net) or whether food is } \\
\text { available without manipulation. } \\
\text { Behavior includes: Investigating with trunk, manipulating and eating } \\
\text { before/after interacting with the object; obvious attempts to reach } \\
\text { and manipulate the object (e.g., the object in a position that is } \\
\text { challenging to reach). }\end{array}$ \\
\hline Feeding/Drinking & $\begin{array}{l}\text { Gathering, manipulating and/or putting any food item in the mouth } \\
\text { without interacting with an object. Drinking water, not bathing. } \\
\text { Level 2: Activity level (stationary, locomoting) } \\
\text { Modifier: Food/water source } \\
\text { Eating: Requires the elephant put something in its mouth; } \\
\text { manipulating alone an chewing alone is not sufficient. }\end{array}$ \\
\hline
\end{tabular}




\begin{tabular}{|c|c|}
\hline Behavior Category & Definition \\
\hline $\begin{array}{l}\text { Enrichment (non- } \\
\text { food) Object } \\
\text { Interaction }\end{array}$ & $\begin{array}{l}\text { Interacting with non-food items that are not permanent (e.g., } \\
\text { firehose ball). } \\
\text { Modifier: Enrichment object }\end{array}$ \\
\hline $\begin{array}{l}\text { Habitat Feature } \\
\text { Interaction }\end{array}$ & $\begin{array}{l}\text { Interacting with features that are permanent in the habitat (e.g., } \\
\text { dusting, bathing, digging, rubbing body, climbing on logs, } \\
\text { investigating features with feet or mouth) } \\
\text { Modifier: Feature }\end{array}$ \\
\hline $\begin{array}{l}\text { Repetitive Behaviors } \\
\text { (Stereotypy) }\end{array}$ & $\begin{array}{l}\text { Motor: Repeatedly performing a behavior for } 3 \text { or more consecutive } \\
\text { repetitions without interruption (e.g., route tracing). } \\
\text { Non motor: Performing the behavior for } 5 \text { seconds or more. } \\
\text { Level 2: Major form of stereotypy } \\
\text { Stereotypy: If a behavior appears to have a purpose (e.g., scooping } \\
\text { small food items in a repetitive manner), it may fit in a different } \\
\text { category. }\end{array}$ \\
\hline Locomotion & $\begin{array}{l}\text { Walking or running (fast walking) more than } 2 \text { body lengths in any } \\
\text { direction, without stopping for } 3 \text { sec or more. } \\
2 \text { body lengths defined as: } 2 \text { adult female body lengths (c.a. } 10 \mathrm{~m} \text { ). }\end{array}$ \\
\hline $\begin{array}{l}\text { Solitary / } \\
\text { Self-Directed }\end{array}$ & $\begin{array}{l}\text { Solitary behaviors that are inactive or do not involve other elephants } \\
\text { (e.g., elimination, rubbing self, masturbation) }\end{array}$ \\
\hline Stationary & $\begin{array}{l}\text { Any stationary state without engaging in another behavior for } 3 \mathrm{sec} \\
\text { or longer. Standing, walking or shuffling (without moving } 2 \text { body } \\
\text { lengths), sitting, kneeling, lying (prone or sternal). } \\
\text { Level 2: Stationary body position (upright, lay down) } \\
\text { Modifier: Location }\end{array}$ \\
\hline
\end{tabular}




\section{Level 2: Social behaviors}

\begin{tabular}{|c|c|c|}
\hline Function & Behavior & Definition and Examples \\
\hline \multirow[t]{7}{*}{ Affiliative } & $\begin{array}{l}\text { Trunk to: head area, } \\
\text { trunk, body, front legs, } \\
\text { mammary glands }\end{array}$ & $\begin{array}{l}\text { Extend trunk tip to (within } 6 \text { inch) or visibly } \\
\text { touches with trunk. } \\
\text { Modifier: Sender/Receiver/Partner (if mutual) }\end{array}$ \\
\hline & Trunk rub & $\begin{array}{l}\text { Move trunk back and forth over body of another } \\
\text { elephant (not for purpose of taking food atop the } \\
\text { body). } \\
\text { Modifier: Sender/Receiver }\end{array}$ \\
\hline & Trunk twine & $\begin{array}{l}\text { Mutual wrapping of trunks. } \\
\text { Modifier: Partner(s) }\end{array}$ \\
\hline & Tail tuck & $\begin{array}{l}\text { Grab tail with trunk and tuck under front leg. } \\
\text { Modifier: Sender/Receiver }\end{array}$ \\
\hline & Lean/Rub & $\begin{array}{l}\text { Lean or rest against or rub against another } \\
\text { elephant while standing, sitting or lying (includes } \\
\text { under another elephant). } \\
\text { Modifier: Partner(s) } \\
\text { Behavior includes: one elephant lying entirely or } \\
\text { partially under another (e.g., calf lying under } \\
\text { mom/auntie, elephants lying against each other, } \\
\text { one elephant standing partially over another } \\
\text { laying down. }\end{array}$ \\
\hline & Nudge & $\begin{array}{l}\text { Gentle head-to-head, head-to-body, body-to- } \\
\text { body contact initiated by one elephant towards } \\
\text { another. } \\
\text { Modifier: Sender/Receiver }\end{array}$ \\
\hline & Greeting & $\begin{array}{l}\text { Coming together, trunk tip to palatal pit (crease } \\
\text { of mouth) + reciprocation. May include ear } \\
\text { flapping, trunk entwining, arousal. } \\
\text { Modifier: Partner(s) }\end{array}$ \\
\hline
\end{tabular}




\begin{tabular}{|c|c|c|}
\hline Function & Behavior & Definition and Examples \\
\hline & $\begin{array}{l}\text { Play OR } \\
\text { Play no contact / Play } \\
\text { solicit / Self-play }\end{array}$ & $\begin{array}{l}\text { Play-contact: Sparring (pushing trunks, tusking, } \\
\text { shoving, wrestling), back and forth nudging, trunk } \\
\text { wrestling, chasing, rolling, climbing on conspecific } \\
\text { (at least one foot on the head or body of another } \\
\text { elephant). } \\
\text { Play-no contact: Clear play with a conspecific } \\
\text { (e.g., ball kicking, head wagging, splashing); play } \\
\text { soliciting with approach and "run" away or other } \\
\text { behaviors that seem to solicit play; other } \\
\text { behaviors that seem like play but you cannot see } \\
\text { the other animal (e.g., sits on rump and throw } \\
\text { back head, chasing wildlife, sliding on the mud). } \\
\text { Modifier: Partner(s) or Unknown (no contact) } \\
\text { Behavior includes: May also include other } \\
\text { behaviors above. }\end{array}$ \\
\hline & Follow or Approach & $\begin{array}{l}\text { Follow: Walk along path of conspecific with } \\
\text { someone in the lead. Distance between animals } \\
\text { almost constant. } \\
\text { Approach: Move to within proximity (1 body } \\
\text { length) of conspecific } \\
\text { Sender=animal that is taking action - animal that } \\
\text { is following or approaching. } \\
\text { Receiver=animal being followed/approached } \\
\text { Modifier: Sender/Receiver } \\
\text { Approach v. Displace v. Claim food/water/object: } \\
\text { Depends on the sequence of events. }\end{array}$ \\
\hline & Share food/water & $\begin{array}{l}\text { Simultaneously at the same food source or } \\
\text { pushing food toward another, or drinking from } \\
\text { the same water source. Requires that focal has } \\
\text { line of sight with partners. } \\
\text { Modifier: Partner(s) } \\
\text { Behavior includes: Consuming food, sharing space } \\
\text { around a food object even if unable to reach the } \\
\text { object. } \\
\text { Behavior does not include: Interacting with a food } \\
\text { object when they cannot see each other (e.g., on } \\
\text { opposite sides of the enrichment tree), Bathing } \\
\text { from the same water source. }\end{array}$ \\
\hline & Share object & $\begin{array}{l}\text { Simultaneously manipulating same object. } \\
\text { Modifier: Partner(s) }\end{array}$ \\
\hline
\end{tabular}




\begin{tabular}{|c|c|c|}
\hline Function & Behavior & Definition and Examples \\
\hline \multirow[t]{4}{*}{ Agonistic } & Claim-food/object & $\begin{array}{l}\text { Approach and take food or object w/in other } \\
\text { elephant's reach, on its body, or in its mouth. } \\
\text { Includes displacing from a feeding/drinking } \\
\text { source when the other elephant is still } \\
\text { eating/drinking. } \\
\text { Modifier: Sender/Receiver }\end{array}$ \\
\hline & Displace-location & $\begin{array}{l}\text { Approach and overtake position of conspecific, } \\
\text { receiver moves at least } 1 \text { body length. Does not } \\
\text { require physical contact. } \\
\text { Modifier: Sender/Receiver } \\
\text { Behavior does not include: Pushing which results } \\
\text { in displacement. }\end{array}$ \\
\hline & Block, resource hold & $\begin{array}{l}\text { Place body between a resource and an } \\
\text { approaching elephant. Holder may turn rump } \\
\text { towards approaching elephant. } \\
\text { Modifier: Sender/Receiver }\end{array}$ \\
\hline & Strike or push & $\begin{array}{l}\text { Strike: Forceful body contact initiated by one } \\
\text { elephant. Includes: } \\
\text { - Head butt (hit with forehead) } \\
\text { - Trunk strike (hit with trunk) } \\
\text { Push: Forceful head-to-head, head-to-body, body- } \\
\text { to-body contact that typically results in receiver } \\
\text { being displaced, or at least it appears there is } \\
\text { intent to displace or control. } \\
\text { Modifier: Sender/Receiver/Partner (if mutual) }\end{array}$ \\
\hline & Sparring & $\begin{array}{l}\text { Mutual striking or pushing with head and trunk, } \\
\text { tusking, driving. } \\
\text { Modifier: Partner } \\
\text { Sparring v. Playing: Depends on the partner and } \\
\text { appearance of aggression. }\end{array}$ \\
\hline & Backup to & $\begin{array}{l}\text { Backup rump first towards another individual's } \\
\text { head, side, or rump, sometimes extending tail to } \\
\text { touch (moving towards another in a submissive } \\
\text { manner). May end in a Rump Present. } \\
\text { Modifier: Sender/Receiver }\end{array}$ \\
\hline
\end{tabular}




\begin{tabular}{|c|c|c|}
\hline Function & Behavior & Definition and Examples \\
\hline & Rump present & $\begin{array}{l}\text { Back rump under the chin of another (submissive } \\
\text { or reproductive, depending on partner and } \\
\text { behavior sequence). } \\
\text { Modifier: Sender/Receiver }\end{array}$ \\
\hline & Tail pull & $\begin{array}{l}\text { Hold tail with trunk and pull or twist. } \\
\text { Modifier: Sender/Receiver }\end{array}$ \\
\hline & Drive & $\begin{array}{l}\text { Use head, tusks, or trunk to push the rear of } \\
\text { another, maintaining contact while both } \\
\text { elephants move. } \\
\text { Drive v. Pre-mount: Determined by whether the } \\
\text { trunk of the sender is over the back of receiver } \\
\text { with an attempt to get in mounting position. } \\
\text { Modifier: Sender/Receiver }\end{array}$ \\
\hline & Threat & $\begin{array}{l}\text { Ears wide, trunk forward, head raised; may } \\
\text { include foot scraping, twitching the tail, head } \\
\text { shaking, or weaving. } \\
\text { Modifier: Sender/Receiver }\end{array}$ \\
\hline & Charge, mock & $\begin{array}{l}\text { Rapidly approach another animal, ears erect, } \\
\text { head high, tail extended, trunk sometimes } \\
\text { extended. Stops short of contact. Often trumpets. } \\
\text { Stops before contact, often in freeze position. } \\
\text { Modifier: Sender/Receiver }\end{array}$ \\
\hline & Charge, real & $\begin{array}{l}\text { Rapidly approach another animal or object, ears } \\
\text { usually close to head, head high, trunk tucked } \\
\text { under head. Often silent. Attempts contact } \\
\text { (strike, push). } \\
\text { Modifier: Sender/Receiver }\end{array}$ \\
\hline & Chase to retreat & $\begin{array}{l}\text { Sender rapidly approaches receiver and receiver } \\
\text { retreats. } \\
\text { Modifier: Sender/Receiver }\end{array}$ \\
\hline & Intervene & $\begin{array}{l}\text { Place body between the sender and receiver in an } \\
\text { agonistic interaction (typically by a dominant } \\
\text { elephant). }\end{array}$ \\
\hline Arousal & Spinning U/D-group & $\begin{array}{l}\text { Spinning, urinating, defecating as a group (usually } \\
\text { in close proximity) or by one's self with no } \\
\text { contact. May include Arousal-other. } \\
\text { Modifier: Partner(s), if any }\end{array}$ \\
\hline
\end{tabular}




\begin{tabular}{|c|c|c|}
\hline Function & Behavior & Definition and Examples \\
\hline & $\begin{array}{l}\text { Pause/freeze, alert } \\
\text { posture }\end{array}$ & $\begin{array}{l}\text { Pause/freeze: No body movement, absolute } \\
\text { stillness, typically with ears erect, sometimes w/ } \\
\text { forefoot raised and still. } \\
\text { Alert posture: Head raised, ears spread, tail } \\
\text { raised, trunk raised. }\end{array}$ \\
\hline & Defensive circle & $\begin{array}{l}\text { Group forms defense circle around an individual } \\
\text { Modifier: Partners }\end{array}$ \\
\hline & Arousal-other & $\begin{array}{l}\text { Foot scrape, tail erect (parallel to ground), tail up, } \\
\text { trunk slap on ground; may include "running" (in } \\
\text { the presence of other elephants). } \\
\text { Does not include: "Running" during play or with } \\
\text { no social context or "running" with an enrichment } \\
\text { object. }\end{array}$ \\
\hline \multirow[t]{3}{*}{ Chemosensory } & $\begin{array}{l}\text { Trunk to: vulva, penis, } \\
\text { anus, back legs, } \\
\text { temporal gland }\end{array}$ & $\begin{array}{l}\text { Extend trunk to within } 6 \text { inch or visibly touches } \\
\text { with trunk. } \\
\text { NOTE: Temporal gland may be difficult to } \\
\text { differentiate in video. Score as Trunk to head area } \\
\text { if it is not clear. } \\
\text { Modifier: Sender/Receiver/Partner (if mutual) }\end{array}$ \\
\hline & Trunk to: urine, feces & $\begin{array}{l}\text { Extend trunk tip to (within } 6 \text { inch) or visibly } \\
\text { touches with trunk the urine or feces, either from } \\
\text { self or another elephant. }\end{array}$ \\
\hline & Flehmen & $\begin{array}{l}\text { Place dorsal trunk finger onto roof of mouth } \\
\text { (VNO openings). }\end{array}$ \\
\hline \multirow[t]{2}{*}{ Reproductive } & Rump present & $\begin{array}{l}\text { Back rump under chin of another (reproductive or } \\
\text { submissive depending on partner and behavior } \\
\text { sequence). } \\
\text { Modifier: Sender/Receiver }\end{array}$ \\
\hline & Pre-mount/Mount & $\begin{array}{l}\text { Pre-mounting (chasing, driving with trunk over } \\
\text { back) or mounting behaviors. } \\
\text { Modifier: Sender/Receiver }\end{array}$ \\
\hline \multirow[t]{2}{*}{ Dam-offspring } & $\begin{array}{l}\text { Nurse/Attempted } \\
\text { nurse }\end{array}$ & $\begin{array}{l}\text { Nursing (dam stands for calf to suckle) or attempt } \\
\text { nursing (dam does not move front leg forward or } \\
\text { dam moves it leg back to block a teat). }\end{array}$ \\
\hline & Claim calf & $\begin{array}{l}\text { Secure space around the calf by physically moving } \\
\text { the calf away from another elephant or blocking } \\
\text { access to the calf. Appears like an act of } \\
\text { protecting or owning. }\end{array}$ \\
\hline
\end{tabular}




\section{Level 2: Feeding/drinking activity level}

\begin{tabular}{|l|}
\hline \multicolumn{1}{|c|}{ Behavior (Activity Level) } \\
\hline Feeding/drinking while stationary \\
\hline Feeding/drinking while locomoting \\
\hline
\end{tabular}

\section{Level 2: Major form of stereotypy}

\begin{tabular}{|c|c|c|}
\hline Major Form & Sub-Form & Definition and Examples \\
\hline \multirow{3}{*}{$\begin{array}{l}\text { Whole-body } \\
\text { stereotypy }\end{array}$} & Limb-swinging & $\begin{array}{l}\text { Swinging a limb in a consistent pattern without body } \\
\text { movement. } \\
\text { - Trunk swinging: swinging the trunk in a repeated } \\
\text { manner. } \\
\text { - Leg swinging: swinging a leg or legs in a repeated } \\
\text { manner. }\end{array}$ \\
\hline & $\begin{array}{l}\text { Forward, } \\
\text { backward, or } \\
\text { vertical } \\
\text { movement }\end{array}$ & $\begin{array}{l}\text { Forward, backward, or vertical body movements } \\
\text { occur along the axis parallel to the elephant's spine. } \\
\text { Does not involve travel. } \\
\text { - } \quad \text { Rocking: the body and head move in unison in a } \\
\text { forward/backward motion with little vertical } \\
\text { movement of the head. } \\
\text { - Bobbing: the head is moved in a vertical motion } \\
\text { and sometimes twisted, turning the chin from } \\
\text { shoulder to shoulder. Bobbing typically } \\
\text { corresponds with vertical elevation and } \\
\text { depression of the spinal column between the } \\
\text { shoulder blades and the pelvic girdle. } \\
\text { Bobbing while rocking: simultaneous } \\
\text { performance of bobbing and rocking. }\end{array}$ \\
\hline & $\begin{array}{l}\text { Side to side } \\
\text { Movement }\end{array}$ & $\begin{array}{l}\text { Body movement occurs along the axis horizontally- } \\
\text { perpendicular to the elephant's spine. } \\
\text { - Swaying: the body and head move counter to } \\
\text { each other, causing the body to undulate } \\
\text { horizontally. } \\
\text { - Weaving: the body undulates side to side while } \\
\text { the head is lifted or twisted vertically. }\end{array}$ \\
\hline $\begin{array}{l}\text { Locomotor } \\
\text { stereotypy }\end{array}$ & $\begin{array}{l}\text { Locomotor } \\
\text { movement }\end{array}$ & Repeatedly walking the same line of travel. \\
\hline
\end{tabular}




\begin{tabular}{|c|c|c|}
\hline $\begin{array}{l}\text { motor: } \\
3 \text { repetitions }\end{array}$ & & $\begin{array}{l}\text { - Pacing: following a relatively straight path in a } \\
\text { forward or backward direction with patterned } \\
\text { turns at each end of the path. } \\
\text { - } \quad \text { Route tracing: following a specific route. Route } \\
\text { tracing patterns are more circuitous and longer } \\
\text { than the straight paths associated with pacing. } \\
\text { Locomotor stereotypy: Criteria is } 3 \text { repetitions of the } \\
\text { same pattern without leaving the path/route or } \\
\text { performing a competing behavior. If the animal } \\
\text { becomes stationary, but does not leave the path, then } \\
\text { resumes pacing or route tracing, this is a continuation } \\
\text { of the same bout. If the animal leaves the path and } \\
\text { then resumes pacing or route tracing, then this starts } \\
\text { a new bout and the criteria of } 3 \text { repetitions starts } \\
\text { over. }\end{array}$ \\
\hline $\begin{array}{l}\text { Self-directed } \\
\text { stereotypy } \\
\text { motor: } 3 \text { reps } \\
\text { non-motor: } 5 \mathrm{sec}\end{array}$ & $\begin{array}{l}\text { Self-directed } \\
\text { behavior }\end{array}$ & $\begin{array}{l}\text { Self-stimulating behaviors. } \\
\text { - Limb or head banging: striking one's limbs or the } \\
\text { head against objects in a non-species typical } \\
\text { manner. } \\
\text { - Nipple pulling: using trunk to pull or manipulate } \\
\text { one's own nipples. }\end{array}$ \\
\hline $\begin{array}{l}\text { Oral stereotypy } \\
\text { non-motor: } 5 \mathrm{sec}\end{array}$ & Oral behavior & $\begin{array}{l}\text { - Trunk sucking or "thumb sucking": sucking or } \\
\text { chewing one's own trunk } \\
\text { (to differentiate from putting something in } \\
\text { mouth). } \\
\text { - Placing foreign object in the mouth and sucking } \\
\text { or chewing on it } \\
\text { (Foreign object = not food. If it's not obvious, } \\
\text { consider it food.) } \\
\text { Bar biting: taking a bar into the mouth and } \\
\text { biting or sucking on it } \\
\text { Chain chewing: taking a chain or cable into } \\
\text { the mouth and biting or sucking on it }\end{array}$ \\
\hline
\end{tabular}




\section{Level 2: Stationary body position}

\begin{tabular}{|l|l|}
\hline \multicolumn{1}{|c|}{ Position } & \multicolumn{1}{c|}{ Definition } \\
\hline Stationary state-Upright & Standing, waling/shuffling, sitting, kneeling. \\
\hline Stationary state-Lay down & $\begin{array}{l}\text { Lying (prone or sternal). Sternum or side is in contact with } \\
\text { substrate; can vary from curled up to stretched out. } \\
\text { Behavior does not include: } \text { Lying under another elephant, } \\
\text { which is social }\end{array}$ \\
\hline
\end{tabular}

Modifier: Location

\begin{tabular}{|l|}
\hline \multicolumn{1}{|c|}{ Location } \\
\hline Gate or Door \\
\hline Under shade structure OR shade kite \\
\hline Other location \\
\hline
\end{tabular}

\section{Modifiers: Example resources at Oregon Zoo}

\begin{tabular}{|c|c|c|}
\hline Food Delivery Object & Habitat Feature & Enrichment Object \\
\hline Timed feeder & Pool-large & Barrel/Keg \\
\hline Enrichment tree & Pool-wading & Firehose ball \\
\hline Cement herd feeder & Drinker (to bathe) & Log, small \\
\hline Habitat enclosure w food & Large logs or rocks & Tire, small \\
\hline $\begin{array}{l}\text { Hanging hay } \\
\text { net/barrel/puzzle }\end{array}$ & Cement herd feeders (to climb) & Boomer ball \\
\hline Barrel/keg with food & Tire, large & Spinner \\
\hline Boomer ball with food & Concrete curb & Pipe puzzle \\
\hline Spinner with food & $\begin{array}{l}\text { Substrate (dirt/mud, sand, gravel, } \\
\text { straw, cedar shavings) or feces }\end{array}$ & Snow \\
\hline \multirow[t]{2}{*}{ Pipe puzzle with food } & $\begin{array}{l}\text { Habitat enclosure (walls, gates, } \\
\text { doors, poles, training wall }\end{array}$ & Water cannon \\
\hline & $\begin{array}{l}\text { Shade structure (active } \\
\text { engagement) }\end{array}$ & Deluge system \\
\hline
\end{tabular}


Appendix D. Mann-Whitney U-test for pairwise comparisons (Chapter 4).

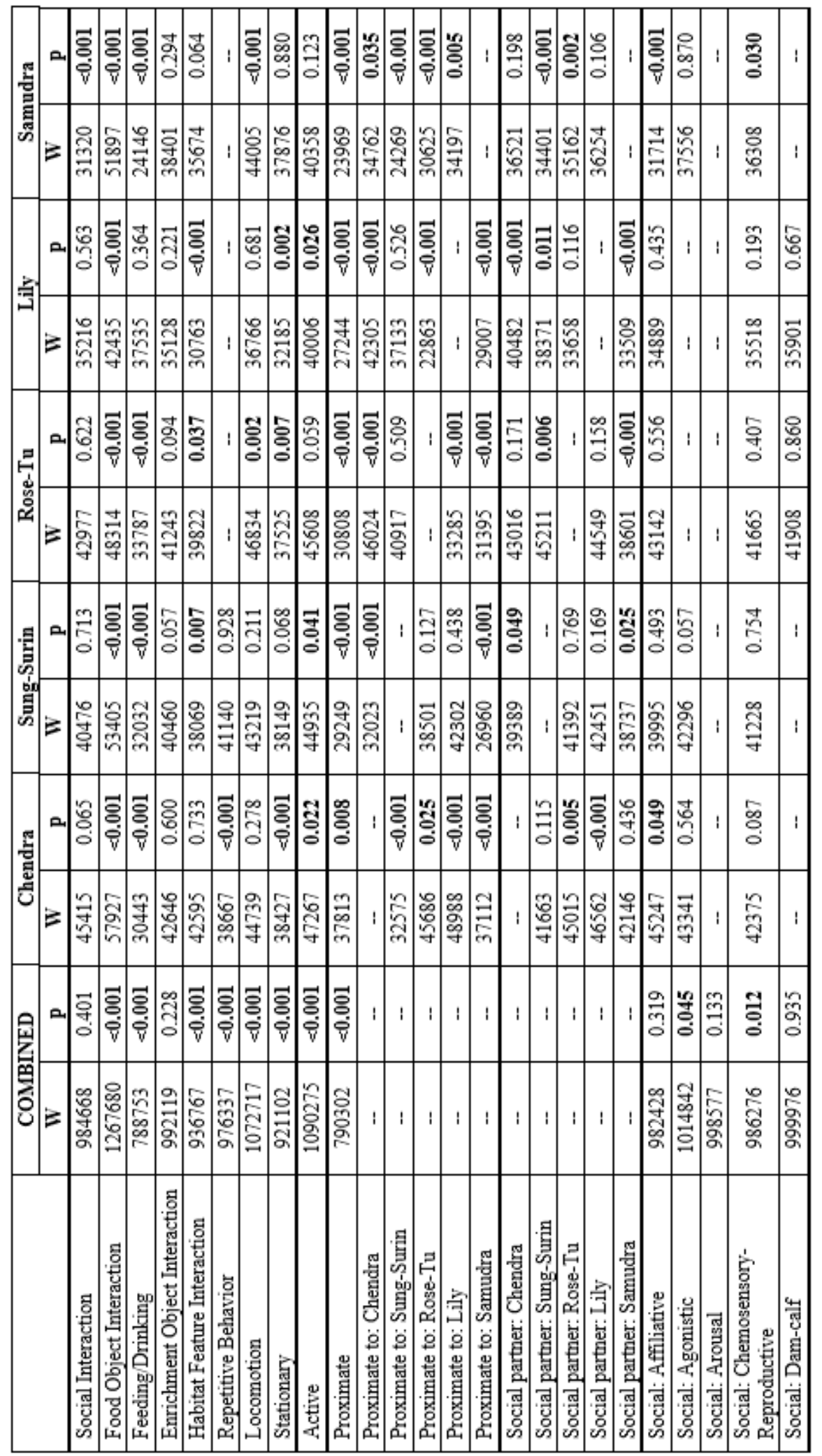




\begin{tabular}{|c|c|c|c|c|c|c|c|c|c|c|c|c|c|}
\hline $\mathfrak{d}$ & & 宮 & 웧 & & & $\stackrel{\circ}{\circ}$ & $\begin{array}{c}5 \\
\vdots \\
0 \\
0 \\
0\end{array}$ & 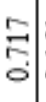 & $\begin{array}{l}\overrightarrow{0} \\
\stackrel{0}{0} \\
\end{array}$ & 1 & 势 & $\begin{array}{l}\text { '。 } \\
\text { o. } \\
\circ\end{array}$ & สิ \\
\hline ค็ & $=\left[\begin{array}{l}\infty \\
0 \\
0\end{array}\right.$ & ్్ㅀ & $\mid$ & 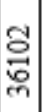 & & 局 & 离 & 吕 & : & 1 & $\begin{array}{l}\infty \\
0 \\
\tilde{n} \\
\tilde{m}\end{array}$ & 鿖 & $\begin{array}{c}\tilde{B} \\
\tilde{n} \\
0\end{array}$ \\
\hline & $\mid \begin{array}{l}\mid \vec{\delta} \\
\hat{\theta}\end{array}$ & 응 & $\mid \begin{array}{l}\text { 索 } \\
\vdots \\
0\end{array}$ & 1 & 혀ํ & 1 & 1 & 1 & 1 & 1 & 1 & ! & 1 \\
\hline 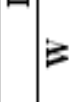 & $=\mid \begin{array}{l}\circ \\
\end{array}$ & స్త్ & 吕 & : & $\mid \begin{array}{l}\vec{n} \\
\text { ने } \\
\text { ने }\end{array}$ & 1 & 1 & 1 & 1 & 1 & 1 & 1 & 1 \\
\hline$\exists$ & $=\frac{E}{0}$ & 응 & & '气 & 형 & 1 & 1 & 1 & 1 & 1 & 1 & ! & 1 \\
\hline 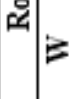 & | & 呑 & & $\tilde{\tilde{f}}$ & $\mid$ & 1 & 1 & 1 & 11 & 1 & 1 & 1 & 1 \\
\hline 园 & $=\mid \overrightarrow{\mathrm{g}}$ & 1 & $\mid \begin{array}{c}2 \\
0 \\
0 \\
0 \\
0\end{array}$ & $\stackrel{\vartheta}{\circ}$ & 芠 & 1 & 1 & 1 & 1 & 1 & 1 & 1 & 1 \\
\hline & $=\vec{\omega}$ & 1 & ఫે & 芯 & $\mid \begin{array}{l}\text { à } \\
\text { के } \\
\text { लn }\end{array}$ & i & 1 & 1 & 1 & 1 & 1 & 1 & 1 \\
\hline : & $=11$ & $\stackrel{\text { 명 }}{\stackrel{0}{\circ}}$ & ¿े. & 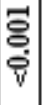 & 穿 & 1 & $\mid \begin{array}{l}6 \\
0 \\
0 \\
0 \\
0\end{array}$ & : & & $\begin{array}{l}\stackrel{a}{0} \\
\stackrel{0}{0} \\
0\end{array}$ & 훙 & $\begin{array}{l}\text { : } \\
\stackrel{\circ}{\circ}\end{array}$ & $\begin{array}{l}\text { J } \\
\circ\end{array}$ \\
\hline 예 & 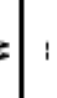 & ฏ̊ & 㝵 & $\begin{array}{l}\text { 誓 } \\
0 \\
\vdots\end{array}$ & 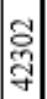 & r & 角 & 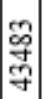 & & $\begin{array}{l}\text { 'े } \\
\text { के } \\
\text { के }\end{array}$ & 离 & 总 & สี \\
\hline 뵴 & 1 & 1 & 1 & 1 & 1 & 1 & 1 & 1 & 1 & 1 & 1 & 1 & 1 \\
\hline 인 & $\geqslant 1$ & 1 & 1 & 1 & 1 & 1 & 1 & 1 & 1 & 1 & 1 & ' & 1 \\
\hline & 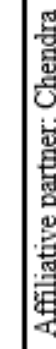 & 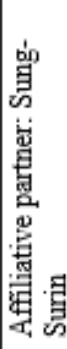 & 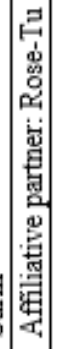 & 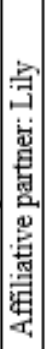 & 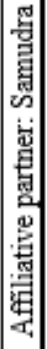 & 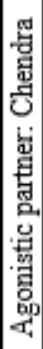 & 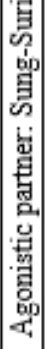 & 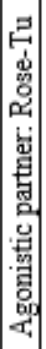 & 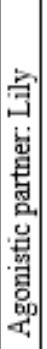 & 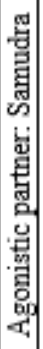 & 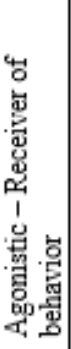 & 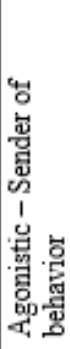 & 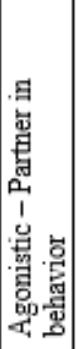 \\
\hline
\end{tabular}

